Mathematischer Korrespondenzzirkel Göttingen (Hg.)

\title{
Voller Knobeleien
}

Erarbeitet von Wolfgang Barthel, Andreas Röscheisen, Karsten Roeseler, Robert Strich, Kristin Stroth und Barbara Zwicknagl

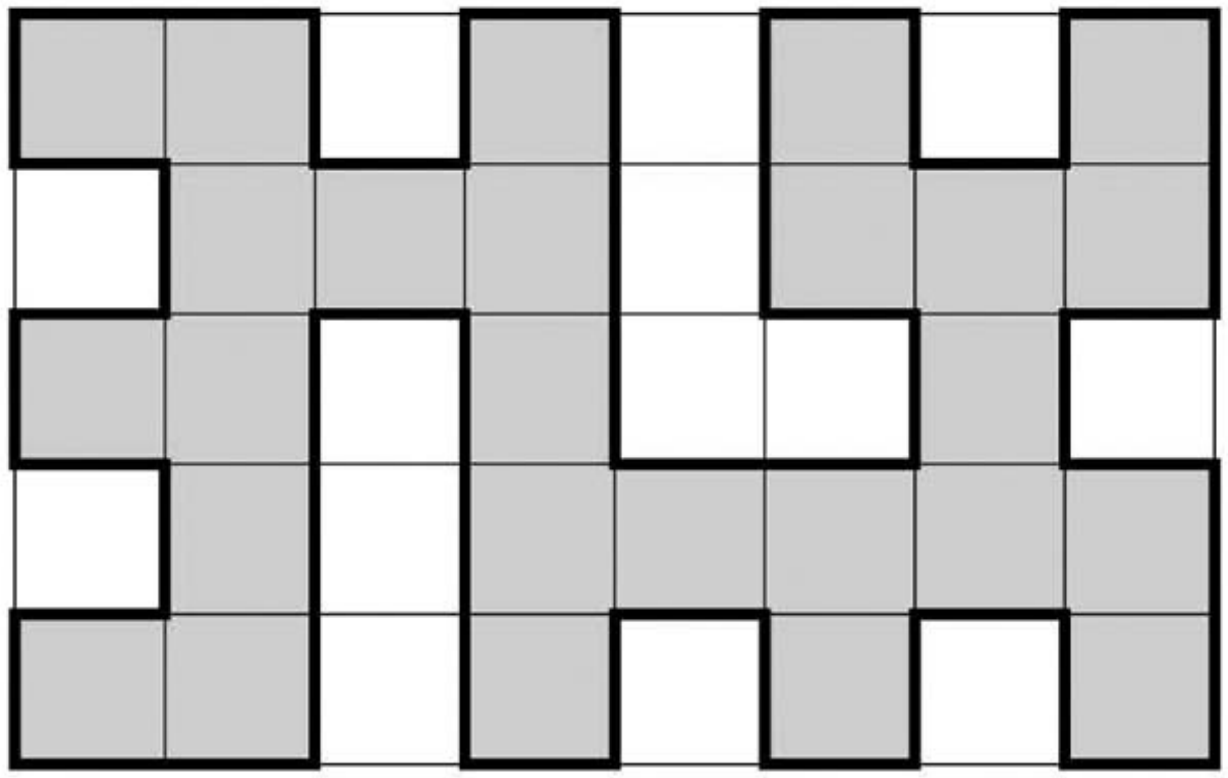



Mathematischer Korrespondenzzirkel Göttingen (Hg.)

Voller Knobeleien 
Erschienen in der Reihe der

Universitätsdrucke Göttingen 2005 
Mathematischer Korrespondenzzirkel Göttingen (Hg.)

Voller Knobeleien

Erarbeitet von Wolfgang Barthel, Andreas Röscheisen, Karsten Roeseler, Robert Strich, Kristin Stroth und Barbara Zwicknagl

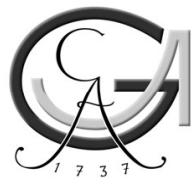

Universitätsverlag Göttingen 2005 
Bibliografische Information der Deutschen Bibliothek

Die Deutsche Bibliothek verzeichnet diese Publikation in der

Deutschen Nationalbibliographie; detaillierte bibliografische Daten sind im Internet über $<$ http://dnb.ddb.de $>$ abrufbar.

Mathematischer Korrespondenzzirkel Göttingen

Mathematisches Institut

Bunsenstraße 3-5

37073 Göttingen

http://www.math.uni-goettingen.de/zirkel/

zirkel@math.uni-goettingen.de

Gedruckt mit freundlicher Unterstützung des Universitätsbundes Göttingen sowie des Mathematischen Institutes der Georg-August-Universität Göttingen

(C) Alle Rechte vorbehalten, Universitätsverlag Göttingen 2005

ISBN 3-930457-76-8 


\section{Vorwort}

Der vorliegende Band beinhaltet eine Zusammenstellung der ersten 25 Aufgabenblätter des Mathematischen Korrespondenzzirkels der Universität Göttingen und der zugehörigen Lösungen.

Der Mathematische Korrespondenzzirkel an der Universität Göttingen entstand vor nunmehr fast fünf Jahren aus einer Initiative der Autoren dieses Buches, einer Gruppe von Studenten, die zum Teil selbst schon während ihrer Schulzeit an derartigen Arbeitsgemeinschaften mit Begeisterung teilgenommen haben. Ziel des Mathematischen Korrespondenzzirkels war und ist die Förderung mathematisch interessierter Schülerinnen und Schüler, insbesondere durch die Bereitstellung mathematischer Aufgaben, die die Zirkelteilnehmer zu Hause bearbeiten können.

Die ersten 100 dieser Aufgaben sind zusammen mit ihren Lösungen im Folgenden abgedruckt.

Die Auswahl dieser Probleme für den Korrespondenzzirkel erfolgte dabei unter anderem unter folgenden drei Gesichtspunkten.

- Die Aufgaben und ihre Formulierung sollen interessant sein.

Die Probleme kommen daher aus einer ganzen Reihe von Themengebieten. Von der Zahlentheorie über kombinatorische Probleme bis hin zur Geometrie findet sich für jeden Geschmack etwas. Auch wurde bei der Formulierung von Aufgaben und Lösungen weniger Wert auf Glanz durch Minimalismus gelegt, sondern es wurde versucht, der jeweiligen Fragestellung sofern möglich einen interessanten Rahmen zu geben.

- Die Aufgaben und ihre Lösungen sollen möglichst nicht schon an vielen anderen Stellen veröffentlicht oder zugänglich sein.

So entstammen viele Probleme der Feder der Autoren. Andere wiederum sind zwar bekannten Aufgaben der Mathematik-Olympiade oder des Bundeswettbewerbs Mathematik entlehnt, wurden aber erweitert oder zumindest umformuliert.

- Die Aufgaben sollen für Schülerinnen und Schüler eines möglichst breiten Altersspektrums zugänglich sein.

Zur Lösung der Aufgaben ist daher nie über die Schulmathematik hinausgehendes Wissen vonnöten; meist genügt Wissen aus der Sekundarstufe I. Innerhalb eines Aufgabenblattes wurde darauf geachtet, dass der Schwierigkeitsgrad der Aufgaben von der ersten zur vierten Aufgabe hin im Regelfall zunimmt - während die erste Aufgabe eines Blattes das Niveau einer leichten Einstiegsaufgabe besitzt, hat die vierte Aufgabe oft den Charakter eines Olympiadeproblems für höhere Klassenstufen. 
Dieses Buch ist geeignet für alle, die Spaß an der Mathematik haben oder auf der Suche nach interessanten mathematischen Kopfnüssen und Herausforderungen sind, insbesondere für Schülerinnen und Schüler, die sich auf mathematische Wettbewerbe wie zum Beispiel die Mathematik-Olympiade vorbereiten wollen. Für Mathematiklehrer und Leiter mathematischer Arbeitsgemeinschaften bietet es ebenso eine reichhaltige Auswahl an Materialien.

Jederzeit dankbar sind wir für alle Hinweise, Anregungen und Ergänzungen zu den Aufgaben und Lösungen in diesem Buch.

Wir wünschen uns, dass die Aufgaben des Mathematischen Korrespondenzzirkels auf diesem Wege weitere Verbreitung finden und vielen Mathematikinteressierten Freude bereiten. 


\section{Über den Korrespondenzzirkel}

\section{Was ist der Mathematische Korrespondenzzirkel?}

Der Mathematische Korrespondenzzirkel ist eine Arbeitsgemeinschaft für Schülerinnen und Schüler, die vom Mathematischen Institut der Universität Göttingen angeboten wird.

\section{Wer kann mitmachen?}

Alle, die Spaß am Lösen mathematischer Probleme haben. Gedacht haben wir an die Klassenstufen 9-13, aber die Teilnahme steht auch jüngeren Schülerinnen und Schülern offen.

\section{Wie funktioniert das?}

Alle sechs Wochen wird von der Uni Göttingen eine Aufgabenserie mit vier mathematischen Aufgaben herausgegeben und direkt an euch geschickt. Außerdem findet ihr die Aufgaben auch im Internet auf unserer Homepage:

$$
\text { http://www.math.uni-goettingen.de/zirkel }
$$

Ihr habt dann ca. vier Wochen Zeit, eure Lösungen an uns zu senden. Diese korrigieren wir, versehen sie mit Anmerkungen und schicken sie euch zusammen mit Lösungsbeispielen und der neuen Aufgabenserie wieder zu.

Einmal im Jahr gibt es ein gemeinsames Treffen am Mathematischen Institut. An diesem Tag habt ihr die Gelegenheit, Uni und besonders Mathe hautnah zu erleben. Außerdem lernt ihr die anderen Zirkelteilnehmer kennen.

\section{Was sind das für Aufgaben?}

Für das Lösen der Aufgaben braucht ihr im Allgemeinen keine über das Schulwissen hinausgehenden Kenntnisse. Es kommt oft eher darauf an, eine pfiffige Idee zu haben. Außerdem gibt es Experimentieraufgaben, bei denen ihr ein wenig herumprobieren, knobeln und selbst eigene Vermutungen aufstellen könnt. Manchmal kann dafür auch ein Computer eingesetzt werden.

\section{Muss ich immer alle Aufgaben lösen?}

Natürlich nicht! Alles, was ihr einsendet, wird von uns angeschaut und korrigiert. Solltet ihr eine Aufgabe nicht vollständig herausbekommen, so könnt (und solltet) ihr auch Ideen und Teilergebnisse aufschreiben. Außerdem gibt es eine Telefon-Hotline für Tipps und Fragen zu den aktuellen Aufgaben.

Die Teilnahme ist kostenlos und unverbindlich und man kann jederzeit aufhören oder auch für eine Aufgabenserie aussetzen. 


\section{Anmeldecoupon}

Haben dir die Aufgaben in diesem Buch gefallen?

Wenn du selbst am Mathematischen Korrespondenzzirkel teilnehmen möchtest, dann kopiere diesen Anmeldecoupon und schicke ihn ausgefüllt an uns. Wir senden dir gerne das aktuelle Aufgabenblatt kostenlos zu.
Name
geb. am
Straße/Nr.
PLZ/Ort
E-Mail

Deine Schule

Schulanschrift

Sonstiges/Bemerkungen (z. B. Teilnahme an Mathematikwettbewerben)
Vorname :

Klasse
$:$

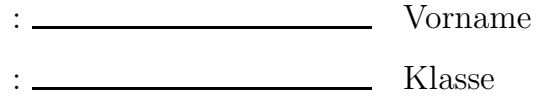

$:$

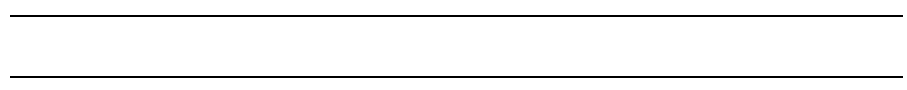

Unsere Anschrift:

Mathematisches Institut

Mathematischer Korrespondenzzirkel

Bunsenstraße 3-5

37073 Göttingen

Internet: http://www.math.uni-goettingen.de/zirkel

E-Mail: zirkel@math.uni-goettingen.de 


\section{Teil I}

Aufgaben 



\title{
Aufgabenblatt 1
}

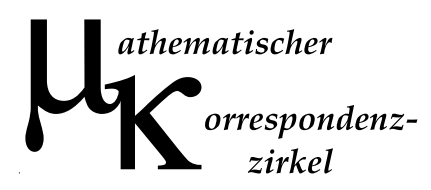

\begin{abstract}
A 1.1 Herr Schulze fährt jeden Tag per Bahn zur Arbeit in die Stadt und kehrt jeden Abend mit demselben Zug zurück, der um 17 Uhr ankommt. Dort am Bahnhof kommt jeden Tag zu genau dieser Zeit auch Herrn Schulzes Chauffeur an, um ihn sofort abzuholen und wieder nach Hause zu fahren.

Eines schönen Tages nimmt Herr Schulze nun einen früheren Zug, der schon um 16 Uhr ankommt. Weil er nicht warten will, geht er seinem Chauffeur entgegen und trifft diesen auch unterwegs, steigt ein, fährt mit ihm nach Hause und kommt dort 20 Minuten früher an als gewöhnlich.

Eines anderen schönen Tages nimmt Herr Schulze einen anderen früheren Zug, der um 16.30 Uhr ankommt. Genau wie beim letzten Mal läuft er seinem Chauffeur schon entgegen, trifft ihn, steigt ein und kommt wieder früher als gewöhnlich zu Hause an. Wie viel früher?

(Bemerkung: Es wird natürlich vorausgesetzt, dass sowohl der Chauffeur als auch Herr Schulze stets mit derselben konstanten Geschwindigkeit fahren bzw. laufen.)
\end{abstract}

A 1.2 Eine Schokoladentafel einer bekannten Marke besteht aus $4 \times 4$ quadratischen Stücken. Sie soll unter 16 Personen aufgeteilt werden, so dass jeder genau ein solches Quadrat bekommt. Dazu muss die Schokolade natürlich in die kleinen Teile zerbrochen werden. Dies soll nun folgendermaßen geschehen: Man nimmt einen vorhandenen Teil der Schokolade, der noch aus mehr als einem einzelnen Quadrat besteht, und macht einen beliebigen Schnitt entlang der Quadratkanten, so dass der Teil in zwei Teile auseinanderbricht. Eine Möglichkeit für den ersten Schnitt wäre also folgende:
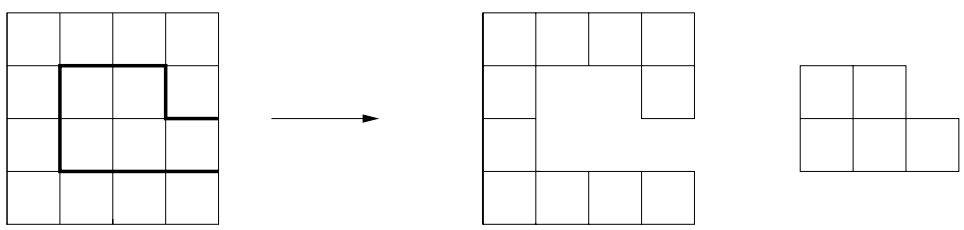

Für das Auseinanderbrechen hat man nun eine Reihe von Möglichkeiten. Wie viele Schnitte muss man dabei mindestens machen? Man versuche die Antwort zu begründen! 
Aufgabenblatt 1

A 1.3 Es gibt Zahlen, die sich nicht ändern, wenn man sie rückwärts liest. So sind zum Beispiel 52325 und 88 oder natürlich auch 3 solche sogenannten Spiegelzahlen oder Palindrome.

Schreibt man alle Palindrome auf, die kleiner als 150 sind, so sieht die Liste folgendermaßen aus:

$1,2,3,4,5,6,7,8,9$

$11,22,33,44,55,66,77,88,99$

$101,111,121,131,141$

Dies sind genau 23 Stück. Mit $P(N)$ sei nun die Anzahl aller Palindrome bezeichnet, die kleiner oder gleich $N$ sind. Also ist zum Beispiel $P(11)=10$ und $P(150)=23$. Man versuche eine Formel für $P(N)$ zu finden und berechne $P(1234567890)$ ! Außerdem zeige man, dass stets $P(N)>2 \sqrt{N}-3$ gilt!

A 1.4 Familie Wurm (Papa, Mama, Kind) hat ein Problem: die Bettdecke von Kindwurm hat einen Riss, und es muss eine neue angeschafft werden. Die Decke soll natürlich die Eigenschaft haben, dass, egal wie sich Kindwurm mit seiner stolzen Länge von genau einem Meter auch räkelt, Kindwurm mit ihr zugedeckt werden kann.

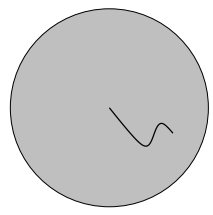

Eine solche Decke könnte zum Beispiel die Form eines Kreises mit Durchmesser $2 \mathrm{~m}$ haben (siehe Bildchen). Denn egal wie Würmchen auch liegt, legt man den Mittelpunkt dieser Decke auf Würmchens eines Ende, so bleibt Wurm vollständig unter der Decke (er ist ja genau einen Meter lang, also so lang wie der Radius der Decke!).

Damit wäre das Problem der Familie Wurm gelöst, wenn nicht Bettdeckenstoff im Wurmland so unglaublich teuer wäre. Langer Rede kurzer Sinn: Familie Wurm will eine Decke mit möglichst kleiner Fläche! Obige Decke hätte nun eine Fläche von ungefähr 3,141593 $\mathrm{m}^{2}$. Kann man das noch verbessern? Jeder Quadratmillimeter zählt, also lohnt es sich, nach kleineren Decken zu suchen! Man versuche eine möglichst kleine Decke zu finden, die aber immer noch Würmchen in jeder Lage abdeckt!

PS: Eine optimale Lösung ist wohl noch nicht bekannt, allerdings ist es nicht allzu schwer, bessere Decken als obige zu finden. 


\title{
Aufgabenblatt 2
}

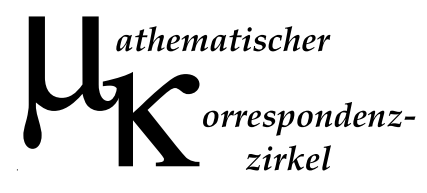

\begin{abstract}
A 2.1 Auf einer Insel sind vier Seeräuber mit einem Schatz aus Goldmünzen gestrandet. Sie beschließen, diesen unter sich aufzuteilen, stellen aber fest, dass dies so nicht geht, weil die Anzahl der Münzen nicht durch 4 teilbar ist. In der Nacht steht nun heimlich einer der Räuber auf, steckt eine Münze ein und kann sich danach genau ein Viertel des Restschatzes nehmen. Damit verschwindet er von der Insel. Kurz darauf erwacht auch der zweite Räuber, ohne den ersten bemerkt zu haben. Auch er muss erst eine Münze entfernen und einstecken, kann danach aber genau ein Viertel des Restschatzes an sich nehmen und heimlich verschwinden. Ebenso passiert es mit Räuber drei und vier, jeder muss erst eine Münze entfernen, bevor er vom Restschatz sein Viertel nehmen kann. Jeder der Räuber glaubt also, etwas mehr als ein Viertel des Schatzes bekommen und seinen schlafenden Kumpanen den Rest zurückgelassen zu haben. Am nächsten Morgen besteht der Schatz noch aus 78 Goldmünzen. Wieviele Münzen waren es ursprünglich?

Zusatzaufgabe: Wären es fünf Räuber gewesen, die auch jeweils eine Münze, dann aber immer ein Fünftel des Schatzes genommen hätten, was wäre dann die kleinste mögliche Anzahl von Münzen im ursprünglichen Schatz?
\end{abstract}

A 2.2 Am Göttinger Fernsehturm soll eine Wendeltreppe installiert werden, auf der man außen am Turm hinauflaufen kann. Der Turm ist bekanntlich 100 m hoch, und die Treppe soll einen konstanten Anstieg haben und den Turm, der einen Durchmesser von 10 Metern hat, genau 20-mal umkreisen. Wenn ein Läufer auf der fertiggestellten Treppe in einem Meter Abstand von der Turmwand nach oben geht, welchen Weg legt er dann dabei zurück?

A 2.3 Es gibt natürliche Zahlen, die gleich der Summe der dritten Potenzen ihrer Ziffern sind. So ist zum Beispiel 153 eine solche Zahl, weil $153=1^{3}+5^{3}+3^{3}=$ $1+125+27$ ist.

a) Zeige, dass es außer der Zahl 1 keine natürliche Zahl gibt, die gleich der Summe der Quadrate ihrer Ziffern ist.

b) Finde alle natürliche Zahlen, die gleich der Summe der dritten Potenzen ihrer Ziffern sind!

Hinweis: Man kann zunächst versuchen zu zeigen, dass solche Zahlen nur wenige Stellen haben können. Danach kann man zum Beispiel systematisch probieren. Auch der Einsatz eines Computers kann hilfreich sein. 
Aufgabenblatt 2

A 2.4 Benötigt werden nur ein Blatt Papier, ein Bleistift und ein Streichholz bzw. eine Nadel oder ein ähnlich geformter Gegenstand. Zur Vorbereitung messe man die Länge $l$ des Streichholzes und male auf das ganze Blatt Papier ein Gitter der Breite $l$, wie es im Bildchen zu sehen ist.

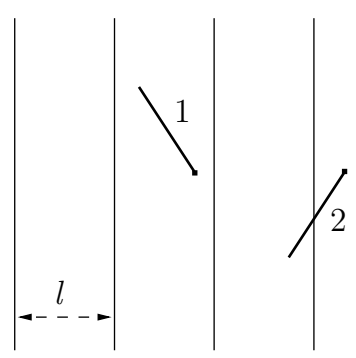

Man werfe das Streichholz nun zufällig einige hundert Mal auf das Gitter und notiere sich dabei, ob das Streichholz eine Linie getroffen hat (Bsp.: Position 1) oder die Linien verfehlt hat (Bsp.: Position 2). „Zufällig“" soll hierbei bedeuten, dass man das Streichholz aus einer gewissen angemessenen Höhe fallen lässt, ohne dabei irgendeine Ausrichtung oder Position zu bevorzugen.

Hat man nun insgesamt $N$-mal geworfen und dabei $T$-mal eine Linie getroffen, so berechne man die relative Trefferhäufigkeit

$$
p=\frac{T}{N} .
$$

Nach genügend vielen Würfen (es sollten durchaus einige hundert sein) sollte diese sich nicht mehr groß ändern. Dann berechne man einmal den Ausdruck

$$
\alpha=\frac{2}{p}
$$

und stelle eine Vermutung an, welcher (bekannte) Wert sich hinter diesem $\alpha$ verbergen könnte! Wie könnte man diese Vermutung beweisen, oder womit könnte das Ergebnis zusammenhängen? 


\section{Aufgabenblatt 3}

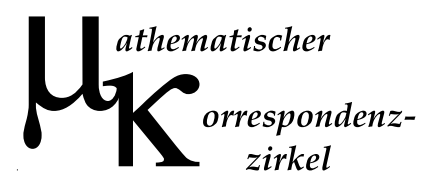

A 3.1 Statistiken besagen, dass unter 1000 Menschen 35 zu hohen Blutdruck haben. Außerdem trinken $80 \%$ der Menschen mit hohem Blutdruck und $60 \%$ der Menschen ohne hohen Blutdruck regelmäßig Alkohol. Wie viel Prozent der regelmäßigen Trinker haben zu hohen Blutdruck?

A 3.2 Natürliche Zahlen (d.h. 1, 2, 3, 4, .. ) kann man mit Rest durcheinander dividieren, z. B. $14: 5=2$ Rest 4 , man sagt „14 lässt bei Division durch 5 den Rest 4".

Welches ist die kleinste natürliche Zahl, die bei Division durch 2 den Rest 1, durch 3 den Rest 2, durch 4 den Rest $3, \ldots$ und durch 10 den Rest 9 lässt?

A 3.3 Gegeben seien ein Kreis $k$ und zwei verschiedene Punkte $A$ und $B$ auf $k$. Man bestimme den geometrischen Ort aller Höhenschnittpunkte von Dreiecken $A B C$, deren dritter Eckpunkt $C$ auf dem Kreis $k$ variiert!

Hinweis: Unter „geometrischem Ort" ist Folgendes zu verstehen: Man wähle sich einige Male auf $k$ einen Punkt $C$ und konstruiere den Höhenschnittpunkt $H$ des Dreiecks $A B C$. Macht man dies für immer mehr Punkte $C$, so bekommt man auch immer mehr Punkte $H$. Alle diese Punkte $H$ bilden eine geometrische Figur, die man auch „geometrischen Ort" nennt.

A 3.4 Man kann auf einem Schachbrett acht Türme so aufstellen, dass sie sich gegenseitig nicht bedrohen, zum Beispiel wie in folgendem Bild:

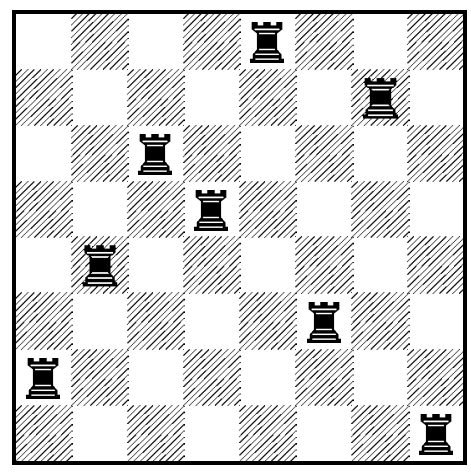

Man versuche zu beweisen, dass man nicht mehr als acht Türme so auf einem Schachbrett platzieren kann, dass sie sich nicht gegenseitig bedrohen! 
Aufgabenblatt 3

Außerdem finde man (mit Begründung!) für jede der Schachfiguren Dame, Läufer, König und Springer jeweils die maximale Anzahl $N$, für die man $N$ derartige Figuren auf einem Schachbrett platzieren kann, ohne dass sie sich gegenseitig bedrohen.

Hinweis: Ein Turm bedroht alle Felder derselben Zeile und derselben Spalte, eine Dame alle Felder derselben Zeile, Spalte und Diagonalen. Ein Läufer bedroht alle Felder derselben Diagonalen. Ein König bedroht alle (maximal acht) direkt benachbarten Felder. Der Springer schließlich bedroht alle von ihm aus im Rösselsprung erreichbaren Felder („zwei vor/zurück, eins zur Seite“ oder „eins vor/zurück, zwei zur Seite"). 


\section{Aufgabenblatt 4}

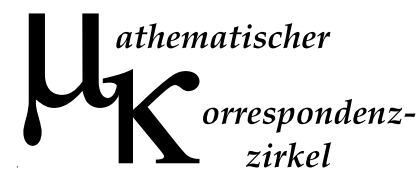

A 4.1 Für ein Verbrechen gibt es vier Verdächtige und jeder von ihnen macht eine Aussage:

- Alfred: „Carlo ist es gewesen!“

- Benno: „Ich war's nicht!“

- Carlo: „Ede hat's getan!“

- Ede: „Carlo hat gelogen, als er sagte, ich wäre es gewesen!“

Angenommen, nur genau einer der vier hat die Wahrheit gesagt. Wer hat dann das Verbrechen begangen? Und angenommen, nur einer der vier hat gelogen, wer war es dann?

A 4.2 Die Pfadfindergruppe Fähnlein Fieselschweif wandert in einer $1 \mathrm{~km}$ langen Schlange mit konstanter Geschwindigkeit durch das Göttinger Umland. Während die Schlange sich so weiterbewegt, läuft Fähnleinführer Tick (mit einer größeren konstanten Geschwindigkeit) einmal vom Ende der Schlange bis zur Spitze, um seine Mannen durchzuzählen, und wieder an seinen Platz am Ende der Gruppe zurück. Als er wieder hinten ankommt, ist die Schlange genau einen Kilometer weiter gewandert. Wie weit ist Tick gelaufen?

A 4.3 Jemand zeichnet ein Dreieck $A B C$ und auf jeder der Dreiecksseiten $B C$, $C A$ bzw. $A B$ noch einen Punkt $P, Q$ bzw. $R$.

Als nun aber noch die drei Umkreise der drei Dreiecke $A R Q, B P R$ und $C Q P$ eingezeichnet werden, stellt dieser jemand überrascht fest, dass sich diese Kreise in einem gemeinsamen Punkt $M$ schneiden. Man wiederhole diese Prozedur an einem eigenen Beispiel noch einmal und versuche zu beweisen, dass sich die erwähnten Kreise immer in einem gemeinsamen Punkt schneiden müssen!

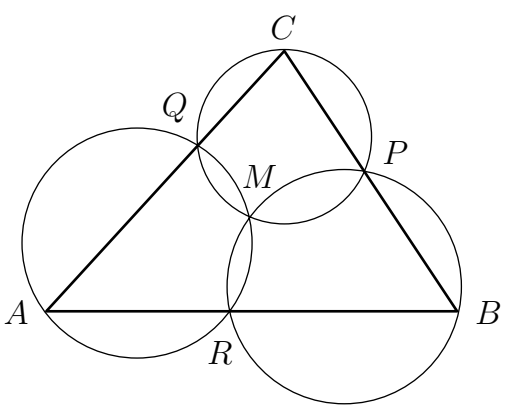


Aufgabenblatt 4

A 4.4 Ein Kohlenkeller hat die Form eines Quadrates der Seitenlänge 1. Drei Familien wollen diesen durch den Einbau von Trennwänden in drei flächengleiche Teile zerlegen. Eine Möglichkeit hierzu ist zum Beispiel die folgende:

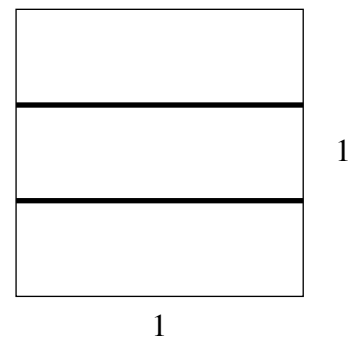

Die Gesamtlänge der eingebauten Wände ist hierbei 2. Trennwände sind nun aber ziemlich teuer und deswegen sollte man versuchen, die Gesamtlänge der Trennwände so kurz wie möglich zu wählen.

Man finde eine solche möglichst kurze Variante! (Die Trennwände müssen hierbei keineswegs immer gerade verlaufen!) 


\section{Aufgabenblatt 5}

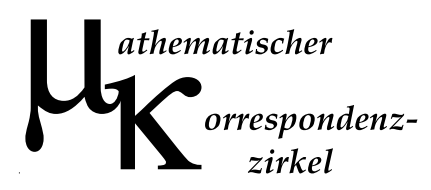

A 5.1 Welche der beiden Zahlen ist die größere:

$$
\frac{7777776}{7777779} \quad \text { oder } \quad \frac{7777777}{7777780} \quad \text { ? }
$$

Hinweis: Die Benutzung von Taschenrechner bzw. Computer verstößt gegen die Mathematikerehre!

A 5.2 Man finde die kleinste natürliche Zahl $n$ mit folgender Eigenschaft:

Streicht man die letzte Ziffer von $n$ und setzt sie vor die erste Ziffer, so entsteht eine Zahl, die genau viermal so groß ist wie $n$.

A 5.3 Eine Frauenstammtischrunde bestehend aus $n$ Personen kommt jeden Freitag zusammen. Jede einzelne von ihnen spielt nun samstags Lotto und man kann natürlich nicht bis zum nächsten Freitag warten, um die Gewinnnachrichten der anderen zu erfahren. So vereinbart man, sich noch am selben Samstagabend über E-Mail auszutauschen, und zwar so, dass in jeder E-Mail die Absenderin alle Informationen weitergibt, die sie bisher erhalten hat. Eine Möglichkeit dazu wäre nun, dass jede jeder genau einmal eine E-Mail schreibt. Man will aber Telefonkosten sparen, also einigt man sich vorher, in welcher Reihenfolge wer wem eine E-Mail schreibt. Was ist die kleinstmögliche Anzahl von zu schreibenden E-Mails, bei der jede der Frauen vom Glück oder Pech jeder anderen erfährt?

A 5.4 Auf kariertem Papier ist folgende Figur aufgemalt:

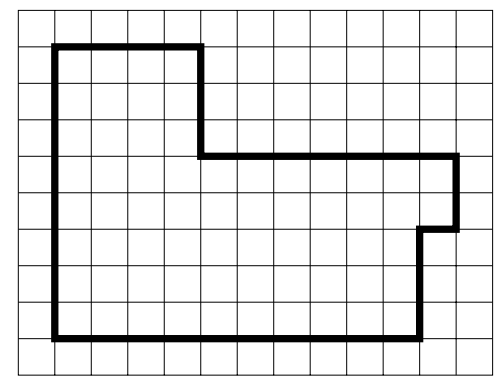

Wenn man sich einmal die Mühe macht, die Kästchen in der Figur auszuzählen, so stellt man fest, dass es genau 64 sind. Das sind bekanntlich $8 \cdot 8$, also sollte es doch möglich sein, die Figur entlang der Kästchenkanten so in genau zwei Teile zu zerteilen, dass man aus ihnen ein Quadrat der Seitenlänge 8 zusammenlegen kann, oder? 



\section{Aufgabenblatt 6}

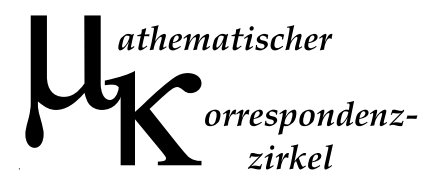

A 6.1 Gibt es eine Quadratzahl, deren Quersumme 6 ist?

Hinweis: Die Quersumme einer Zahl ist die Summe ihrer Ziffern!

A 6.2 Beim Göttinger Altstadtfest sollen, wie in der Abbildung angedeutet, zwei Schmuckbänder zwischen zwei Bäumen aufgehängt werden, und zwar jeweils von der Spitze des einen Baums zum unteren Stammende des anderen Baums.

Die Organisatoren des Festes sind nun ein wenig besorgt, denn es soll unter den Bändern wenigstens noch so viel Platz bleiben, dass ein Mensch darunter durchgehen kann. Zwar sind die Höhen der Bäume erst kürzlich bestimmt worden, nämlich $x=$ $6 \mathrm{~m}$ und $y=4 \mathrm{~m}$, aber der Abstand $d$ der Bäume ist völlig unbekannt.

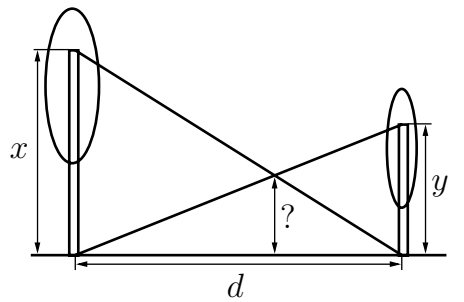

Muss man hierzu dem Vermessungsamt einen Auftrag erteilen oder kann man die Organisatoren vorher schon beruhigen?

A 6.3 Bei dem Zahlenschloss an Peters Fahrrad muss man durch Drehen dreier Rädchen, auf denen jeweils die Ziffern 1, 2 und 3 stehen, einen dreiziffrigen Zahlencode einstellen, um das Schloss zu öffnen.

Angenommen, das Schloss ist defekt und öffnet sich schon, wenn beliebige zwei der drei Ziffern richtig eingestellt sind, wie viele Versuche braucht ein potentieller Dieb dann höchstens (wenn er schlau ist!), um das Schloss zu öffnen?

(Tipp: Die richtige Antwort ist nicht neun.)

A 6.4 Man nehme den Prospekt eines beliebigen Supermarktes, Möbelhauses oder Computerladens. Jedes Produkt hat dort seinen Preis - und um diese Preise soll es in dieser Aufgabe zunächst einmal gehen.

Schaut man sich diese Zahlen nämlich genauer an, so stellt man in der Regel fest: Die meisten Preise haben als letzte Ziffer eine 9, einige wenige vielleicht eine 0 oder 5, aber andere Ziffern kommen so gut wie gar nicht vor.

Doch in dieser Aufgabe soll nicht die letzte, sondern die erste Ziffer der Zahlen eine Rolle spielen. Uns interessiert folgende Frage:

Kommen die Zahlen 1, 2, 3, 4, 5, 6, 7, 8 und 9 gleich oft als erste Ziffer vor oder nicht?

Für die letzte Ziffer haben wir die Frage oben beantwortet, da kommt die 9 eindeutig häufiger vor. Hast du eine Vermutung aufgestellt? Dann solltest du sie überprüfen! 
Aufgabenblatt 6

Fertige dir dazu eine Tabelle (zum Beispiel) nach folgendem Muster an:

\begin{tabular}{c|l|l|l|l|l|l|l|l|l|l} 
Preise mit 1. Ziffer & 1 & 2 & 3 & 4 & 5 & 6 & 7 & 8 & 9 & Gesamtzahl \\
\hline \hline ALBI-Prospekt & 71 & 61 & 40 & 31 & 20 & 16 & 18 & 13 & 18 & 288 \\
\hline in Prozent & 24,6 & 21,2 & 13,9 & 10,7 & 6,9 & 5,5 & 6,3 & 4,6 & 6,3 & 100 \\
\hline \hline $\begin{array}{c}\text { Opi-Baumarkt } \\
\vdots\end{array}$ & \multicolumn{10}{c|}{$\vdots$}
\end{tabular}

Wichtig sind zwei Dinge:

- Nullen am Anfang zählen nicht. Das heißt z. B., die erste Ziffer von 0,49€ ist eine 4.

- Die Anzahl der gezählten Preise muss möglichst groß sein, also etwa zwischen 250 und 1000 liegen. Das geht mit einer Strichliste ziemlich schnell.

Erkennst du eine Gesetzmäßigkeit? Warum sollte die nur auf Werbeprospekte beschränkt sein? Überlege, wo einem im Alltag noch so Zahlen begegnen und füge noch ca. 3 bis 5 (oder mehr) Zeilen in die Tabelle. Ein paar Ideen: Sportseite der Montagszeitung, Weltatlas (Einwohnerzahlen von Städten, Fläche von Ländern oder Seen), Größe von Dateien auf einer Computerfestplatte, Lottozahlen, Gewinnquoten, ...

a) Welche Beobachtungen hast du gemacht (Tabelle!)?

b) Stelle die Tabellenwerte grafisch dar! Trage auf der $x$-Achse die Ziffern $(1,2$, $\ldots, 9)$, auf der $y$-Achse die zugehörigen prozentualen Häufigkeiten auf.

c) Gibt es eine Gesetzmäßigkeit? Kennst du eine Funktion, mit der man die Punkte der Graphen verbinden kann?

d) Warum treten die Zahlen in der Umwelt so auf, wie du es beobachtet hast? (Man kann das mathematisch begründen.) 


\section{Aufgabenblatt 7}

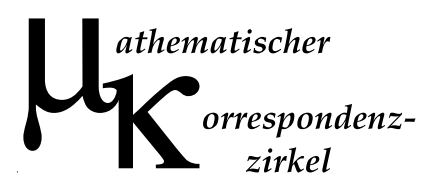

A 7.1 Die handelsüblichen Papierformate DIN A0, DIN A1 usw. haben folgende praktische Eigenschaften:

1. Die Seitenverhältnisse verschiedener Formate sind immer dieselben. Das heißt, wenn zum Beispiel $a_{3}$ und $b_{3}$ (mit $\left.a_{3}>b_{3}\right)$ die Seitenlängen eines DIN-A3Blattes sind und $a_{5}$ und $b_{5}$ (mit $\left.a_{5}>b_{5}\right)$ die Seitenlängen eines DIN-A5Blattes, so verhält sich $a_{3}$ zu $b_{3}$ wie $a_{5}$ zu $b_{5}$.

2. Halbiert man ein Blatt vom Format DIN A $(k)$, so erhält man zwei Blätter vom Format DIN A $(k+1)$ (das $k$ ist hierbei ein Platzhalter für die Zahlen 0,1,2,3 usw.).

3. Ein Blatt vom Format DIN A0 hat eine Fläche von genau einem Quadratmeter.

Bestimme aus diesen Angaben das Seitenverhältnis im DIN-Format, die Seitenlängen eines DIN-A0-Blattes und die Seitenlängen eines DIN-A4-Blattes. Überprüfe das letzte Ergebnis durch direktes Nachmessen!

A 7.2 Zwei Bauarbeiter schaufeln Sand. Schaufelt jeder von ihnen nacheinander die Hälfte des Sandes, so benötigen sie dafür insgesamt 25 Stunden. Wenn sie aber gleichzeitig schaufeln, so schaffen sie diese Arbeit in nur 12 Stunden.

Wie lange würde jeder der beiden allein für den gesamten Haufen benötigen?

A 7.3 Auf einem Zahlenstrahl sitzt ein $n$-Frosch (das ist ein Frosch mit einer besonderen Vorliebe für die Zahl $n$ ).

Er bewegt sich auf dem Zahlenstrahl nach folgender Regel:

Sitzt ein $n$-Frosch auf der Zahl $m$ und ist $m>n$, so macht der $n$-Frosch einen $n$ Sprung nach links und landet auf der Zahl $m-n$. Ist aber $m<n$, so ändert der Frosch seine Vorliebe, wird zum $m$-Frosch und springt zum Abschied auf die Zahl $n$. Ist schließlich irgendwann $m=n$, so ist der $n$-Frosch glücklich und bleibt sitzen.

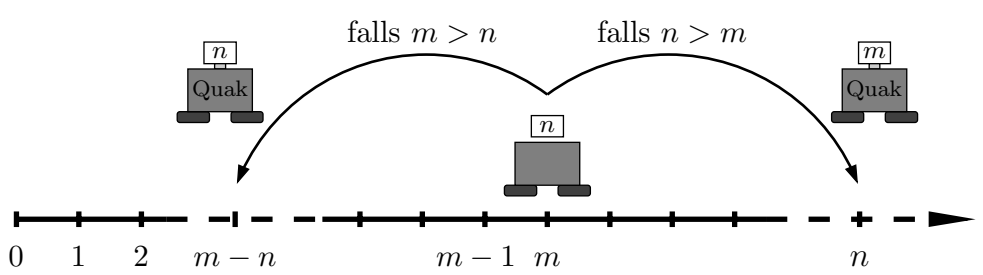


Aufgabenblatt 7

Beschreibe den Weg eines 21-Frosches, der zu Beginn auf der Zahl 12 sitzt, und den eines 35-Frosches, der auf der 11 startet!

Versuche noch weitere Beispiele und stelle eine Vermutung auf, wo ein $a$-Frosch landet, wenn er auf $b$ startet! Beweise diese Vermutung!

Wo also landet ein 1000 000-Frosch, wenn er zu Beginn auf 123456789 steht?

A 7.4 Lege auf einen Tisch von den Spielkartenfarben Karo, Herz, Pik und Kreuz jeweils den Buben, die Dame, den König und das Ass.

Ist es möglich, diese Karten so quadratisch anzuordnen, dass in jeder Reihe und in jeder Spalte jede Farbe und jedes Bild genau einmal vorkommen? 


\section{Aufgabenblatt 8}

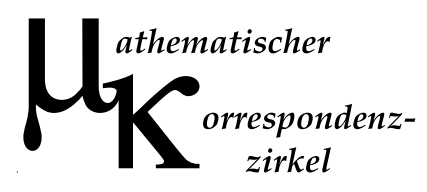

A 8.1 Jedes Mal, wenn Oma Piepenbrink ihre 17 Enkel zu Besuch hat, verteilt sie an diese Taschengeld. Hierzu bereitet sie immer 17 Umschläge vor, und zwar so, dass in einem von ihnen 1 Euro, in einem anderen 2 Euro, ... und im letzten 17 Euro sind. Äußerlich sind die Umschläge jedoch nicht zu unterscheiden, so dass die Enkel zufällig einen Umschlag ziehen.

Nach vier solchen Besuchen stellen die Enkel fest, dass jeder von ihnen insgesamt eine ungerade Anzahl an Euro bekommen hat. Ist das möglich oder hat sich da einer der Enkel verzählt?

A 8.2 a) Eine Leiter mit Länge $\sqrt{8}$ Meter lehnt an der Wand, wobei sie einen 1 Meter tiefen und 1 Meter hohen Tisch gerade an der oberen Tischkante berührt. Der Tisch steht direkt an der Wand (siehe Skizze). Kann man aus diesen Angaben ermitteln, wie hoch über dem Boden die Leiter die Wand berührt?

b) Nun wird die Leiter ausgezogen und hat eine Länge von 6 Metern. Sie lehnt in der gleichen Weise wie oben an Tisch und Wand. Wie hoch über dem Boden berührt die Leiter die Wand

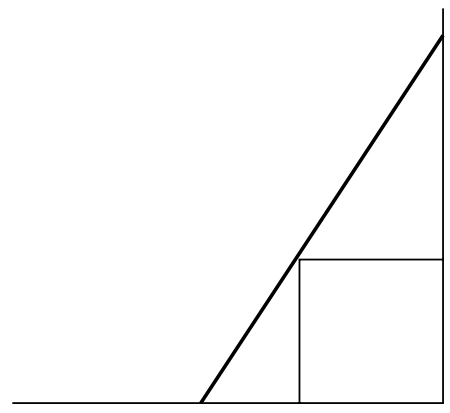
jetzt?

A 8.3 Auf einem Tisch liegen 17 rote, 20 blaue und 24 schwarze Spielsteine. Außerdem ist noch ein genügend großer Vorrat an Spielsteinen aller Farben vorhanden, um folgendes Spiel zu spielen: In jedem Zug darf der Spieler zwei beliebige Steine verschiedener Farbe vom Tisch nehmen, muss dafür aber einen Stein der dritten Farbe neu auf den Tisch legen.

Das Spiel endet also, sobald nicht mehr zwei Steine verschiedener Farbe auf dem Tisch sind. Frauke erzählt nun ihrem Freund Peter, sie habe dieses Spiel gespielt, und dabei sei genau ein Stein auf dem Tisch übrig geblieben. Daraufhin konnte Peter ihr sofort sagen, welche Farbe dieser Stein hatte. Wie konnte er das wissen? Welche Farbe hat er ihr also genannt?

A 8.4 Herr Meier will auf seinem Grundstück vor seinem Haus neuen Rasen pflanzen. Die Rasenfläche, die bepflanzt werden soll, hat die Form eines Quadrates, dem an zwei gegenüberliegenden Seiten jeweils zwei kleinere Quadrate herausgeschnitten wurden (siehe Abbildung). 


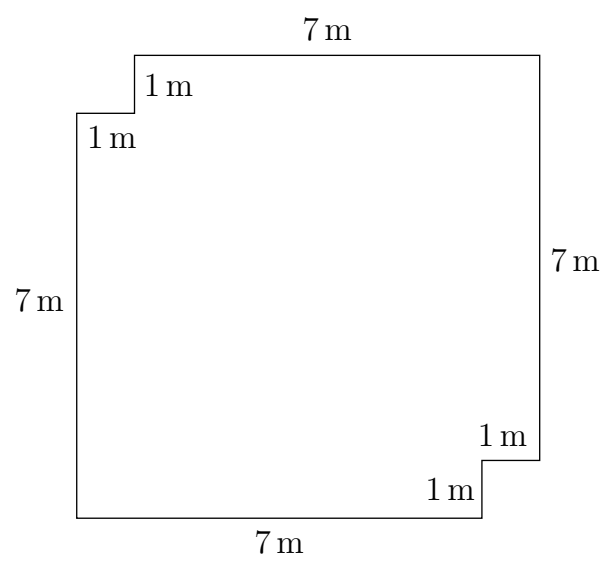

Im Baumarkt hat Herr Meier zu diesem Zweck 31 Grassoden zu je $2 \mathrm{~m} \times 1 \mathrm{~m}$ gekauft und sich vom Verkäufer versichern lassen, dass er damit sein Rasenstück, ohne die Soden noch weiter zerschneiden zu müssen, auslegen kann. Herr Meier versucht an jenem Nachmittag stundenlang ergebnislos, die Rasensoden auf der Fläche zu verteilen.

Erst als seine Frau, die Mathematikerin ist, sich die Sache genauer ansieht, kommt es zu einer Klärung. Sie empfiehlt ihrem Gatten, sich im Baumarkt zu beschweren. Welche Begründung gibt sie ihm mit auf den Weg? 


\section{Aufgabenblatt 9}

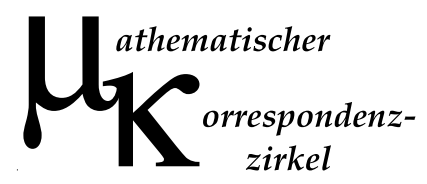

A 9.1 Frank verwechselt an seiner Armbanduhr gelegentlich den großen mit dem kleinen Zeiger und liest dennoch eine sinnvolle Uhrzeit ab. Wie oft am Tag und wann ist dies möglich?

A 9.2 Man beweise, dass $4 n^{3}+6 n^{2}+4 n+1$ für keine natürliche Zahl $n$ eine Primzahl ist!

A 9.3 Immer wenn die sieben Zwerge morgens auf Arbeit in den Wald gehen, laufen sie in einer Reihe hintereinander und singen dazu ihr fröhliches Lied. Die Zwerge, die alle verschieden groß sind, gehen dabei immer so, dass von drei aufeinander folgenden Zwergen der mittlere jeweils größer oder kleiner als die beiden anderen ist. Ihre Reihe ist also immer von der Form hoch - tief - hoch - ... oder tief - hoch - tief - ...

Wie viele Tage können die Zwerge so auf Arbeit gehen, ohne dass sich eine Reihenfolge wiederholt?

Wie viele Tage sind es, wenn sich auch noch Schneewittchen in die Schlange einreiht? Hinweis: Schneewittchen ist natürlich größer als jeder Zwerg!

A 9.4 Bekanntlich kann man eine als unendlich groß angenommene Fläche vollständig und überschneidungsfrei mit kongruenten Rechtecken und auch mit kongruenten Parallelogrammen pflastern (siehe Abbildung!).
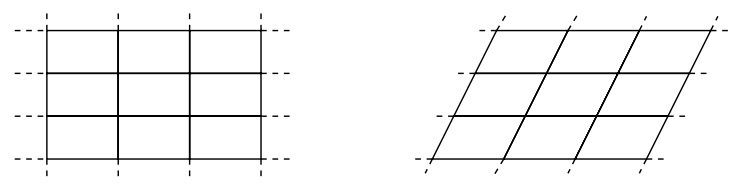

Man beweise, dass dies auch mit kongruenten Vierecken beliebiger Form möglich ist! 



\section{Aufgabenblatt 10}

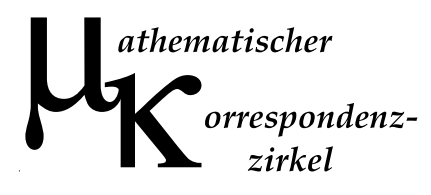

A 10.1 Frau von Schluckspecht sucht ihren Mann, der sich ganz gut in den neun Kneipen der Stadt auskennt: Von vier Abenden, die er nicht zu Hause ist, verbringt er durchschnittlich drei dort, wobei er keine dieser Kneipen bevorzugt.

Nun ist er also wieder mal nicht zu Hause, und nachdem seine Frau schon in acht der Kneipen erfolglos nach ihm gesucht hat, fragt sie sich, wie groß die Wahrscheinlichkeit ist, ihn in der letzten zu finden. Also: Wie groß ist sie?

A 10.2 Bestimme alle Quadratzahlen, die im Dezimalsystem mit lauter gleichen Ziffern geschrieben werden!

A 10.3 Tom ist ein richtiger Weiberheld. Momentan hat er neun Freundinnen (Antonia, Bertha, Claudia, Dora, Emilia, Friederike, Gerda, Hannelore und Ida; kurz: A, B, C, D, ..., I), die alle in derselben langen, schnurgeraden Straße wohnen. Etwa so:

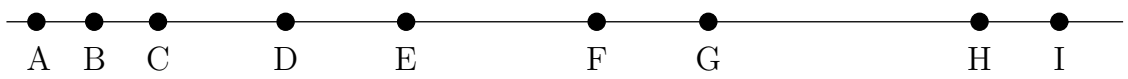

Da er jedes seiner Mädels oft besucht, möchte er jetzt auch in diese Straße ziehen. Wo sollte Tom sich seine Wohnung suchen, damit die Summe der Weglängen von ihm zu jeder der Freundinnen möglichst kurz ist?

Hinweis: Während die Reihenfolge, in der die Mädchen in der Straße wohnen, bekannt ist (siehe oben), sind die genauen Abstände der Häuser nicht bekannt. Die Skizze oben dient nur der Orientierung!

A 10.4 Sind Kassetten eigentlich „optimal" gebaut? Eine normale Musikkassette ist $100 \mathrm{~mm}$ breit und die leeren Spulen haben einen Durchmesser von $21 \mathrm{~mm}$. Ist die Position der Spulen optimal in dem Sinne, dass man bei dem in der Breite gegebenen Platz maximal viel Band in der Kassette unterbringen kann? Rechnet die optimale Position aus (wobei natürlich nach wie vor die Spulen nebeneinander liegen sollen) und vergleicht das Ergebnis mit den tatsächlichen Werten! Versucht, eventuelle Abweichungen zu erklären - es sollte doch verwundern, wenn sich die Erfinder der Kassette nicht solche Gedanken gemacht hätten! Wer Lust hat, kann dieselbe Untersuchung auch an einer Videokassette durchführen.

Tipps: Hilfreich ist es, bei den Berechnungen zwei Eckwerte zu betrachten: Zum einen, wie viel Band Platz hat, wenn es vollständig auf einer Spule aufgewickelt ist, zum anderen, für wie viel Band Platz ist, wenn die Kassette genau zur Hälfte abgespielt worden ist. Die Länge des Bandes kann man dabei schwerlich direkt 
Aufgabenblatt 10

betrachten; aber man kann mit einem äquivalenten Maß rechnen ...

Um das Problem deutlicher zu machen, sei eine nicht optimale Anordnung gezeigt: Ist der Abstand $a$ des Spulenmittelpunkts vom Rand nur $20 \mathrm{~mm}$, so kann eine voll aufgewickelte Spule maximal $40 \mathrm{~mm}$ breit sein. Dann sind in der Mitte aber $20 \mathrm{~mm}$ der Breite nie von einer Spule bedeckt, man hat also Platz verschenkt.

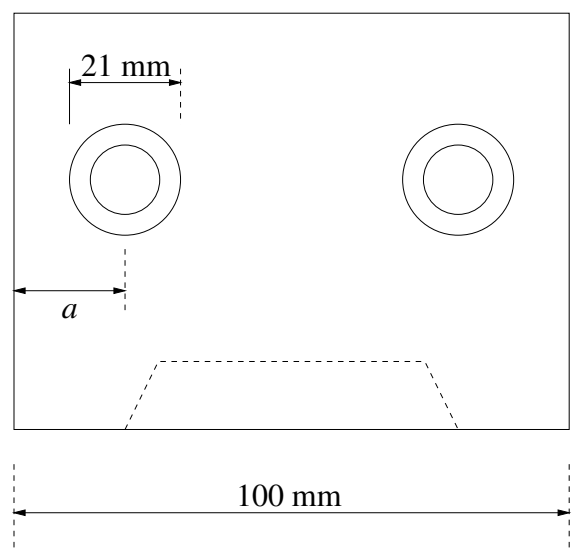




\section{Aufgabenblatt 11}

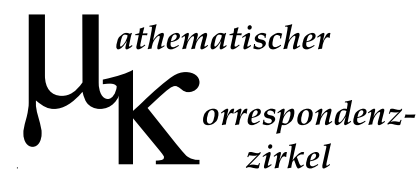

A 11.1 In dem folgenden Diagramm steht jeder Buchstabe für eine der Ziffern $0,1, \ldots, 9$, wobei verschiedene Buchstaben auch verschiedene Ziffern bezeichnen. Finde alle möglichen Lösungen, für die sämtliche Gleichungen erfüllt sind!

$$
\begin{array}{ccccc}
N E N A-B A L L & = & \text { OH } \\
: & - & - & - \\
E N+ & B A S E & = & B E T T \\
= & & = & = \\
R A N * & B A & = & T E E B
\end{array}
$$

A 11.2 Fahrradkurier Rudi Hurtig ist wieder in Neu Weende unterwegs. (Der hier wesentliche Stadtplanausschnitt ist unten abgebildet.) Er muss ein Dokument von $A$ nach $B$ bringen und möchte dies selbstverständlich auf dem kürzestmöglichen Weg erledigen. Wie viele Möglichkeiten hat er dafür? Und wie lang ist dieser Weg dann?

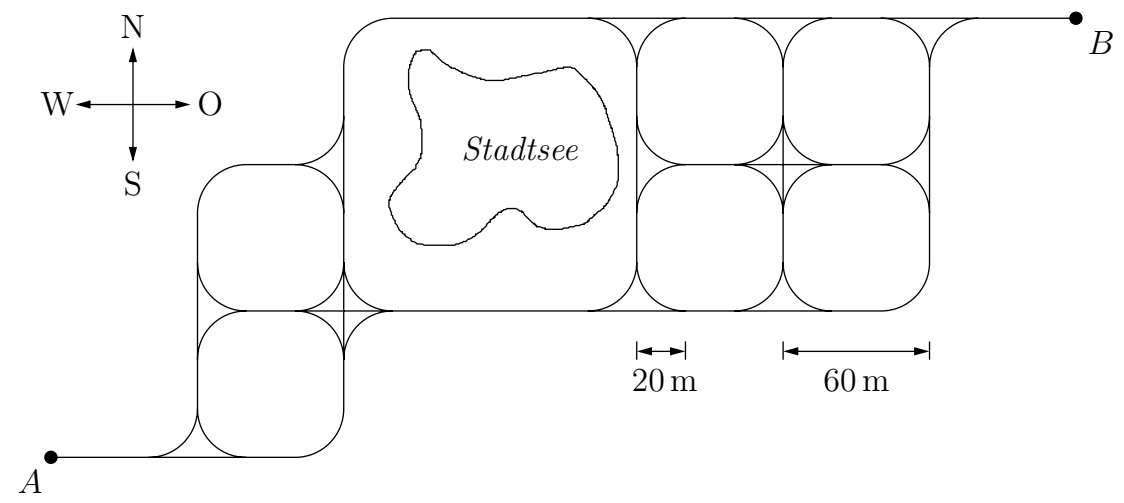

Anmerkung: Die Wege in Neu Weende sind nicht allzu breit (es fahren keine Autos), so dass man sie getrost als Striche zeichnen und auch so rechnen darf. Die Gitterbreite des Quadratgitters, auf dem die Wege angelegt wurden, beträgt $60 \mathrm{~m}$. Die Abbiegekurven zum angenehmeren Abbiegen haben sämtlich einen Radius von $20 \mathrm{~m}$. 
Aufgabenblatt 11

A 11.3 Yvonne und Zacharias spielen ein Hölzchenspiel.

a) Sie haben einen Haufen mit 100 Hölzchen und ziehen abwechselnd mindestens ein und höchstens sieben Hölzchen, wobei Yvonne beginnt (Ladies first). Verloren hat, wer das letzte Hölzchen ziehen muss.

Wer von den beiden kann den Sieg erzwingen?

b) Jetzt spielen sie mit zwei Haufen à 100 Hölzchen, wobei ein Spieler wieder ein bis sieben Hölzchen von einem der zwei Haufen zieht. Wieder darf Yvonne anfangen und wieder verliert derjenige, der das letzte Hölzchen ziehen muss.

Wer kann diesmal den Sieg erzwingen?

A 11.4 Nachtwächter Paul Sorglos soll im Museum für Moderne Kunst den Raum mit den wertvollsten Bildern beaufsichtigen. Der Raum hat nur gerade Wände, ist aber von einem dieser "modernen Architekten“ entworfen und hat somit sehr viele Ecken und ist sehr verwinkelt. Paul Sorglos sitzt nun jeden Abend auf einem Stuhl und beobachtet von dort aus, was rundherum passiert.

Obwohl Paul in einer der Nächte nicht eine Sekunde unaufmerksam war, fehlen am nächsten Morgen Bilder, und zwar von jeder Wand des Raumes eines.

Ihm wird vorgeworfen, dass er die Diebe gesehen und daher mit ihnen zusammengearbeitet haben muss. Aber Paul betont, dass er von seinem Sitzplatz aus die nun fehlenden Bilder gar nicht sehen konnte. Die folgenden Untersuchungen ergeben, dass Pauls Aussage tatsächlich stimmt.

Zeichne einen Grundriss eines Raumes, bei dem dies möglich ist, und markiere einen möglichen Sitzplatz. 


\title{
Aufgabenblatt 12
}

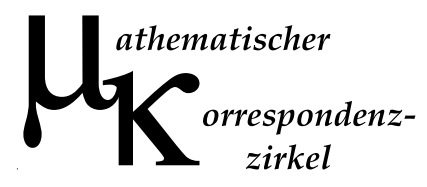

\begin{abstract}
A 12.1 Bei einem Spiel mit drei Spielern muss der Verlierer einer Runde die Punktzahlen der anderen beiden verdoppeln und verliert dabei selbst die entsprechende Zahl an Punkten. Nach drei derartigen Runden hat jeder der drei Spieler genau einmal verloren und jeder hat genau 24 Punkte.

Wie viele Punkte hatten die Spieler zu Beginn?
\end{abstract}

A 12.2 Peter hat einen Schrebergarten von der Form eines gleichseitigen Dreiecks. Statt durch Zäune ist der Garten durch Obststräucher begrenzt. Entlang der ersten Seite wachsen Himbeersträucher, entlang der zweiten Brombeeren und die dritte Seite ist mit Johannisbeeren bepflanzt. An einem sonnigen Sommernachmittag möchte er sich in seinem Garten in seinen Liegestuhl legen und faulenzen. Zwischendurch will er sich auf dem kürzesten Weg ein paar Himbeeren holen und diese im Liegestuhl essen. Wenn er die Himbeeren verspeist hat, möchte er sich genauso Brombeeren holen und später holt er sich noch Johannisbeeren. Da es recht heiß ist, möchte sich Peter aber insgesamt möglichst wenig bewegen, sprich: einen möglichst kurzen Weg zurücklegen.

Peter überlegt sich also vorher, wo er seinen Liegestuhl aufstellen muss.

Kannst du ihm helfen? Wo muss Peter seinen Liegestuhl aufstellen, damit seine Wandertour möglichst kurz wird?

Bemerkung: Der Weg von der Gartentür zum Liegestuhl und der Rückweg am Schluss nach Hause zählen nicht mit!

A 12.3 Wir haben ausreichend viele Perlen in drei Farben zur Verfügung, aus denen wir Armbänder herstellen wollen. Für ein solches Armband fädeln wir insgesamt fünf Perlen auf einen Faden und verbinden die Enden so, dass der Knoten nicht auffällt. Ein so entstandenes Armband hat natürlich keine spezielle Vorderoder Rückseite und auch keinen Anfang und kein Ende.

Wie viele verschiedene Armbänder können wir auf diese Weise herstellen? 
Aufgabenblatt 12

A 12.4 Unten stehendes Bild zeigt eine (stark vereinfachte) Karte von Deutschland. Es gibt immer wieder Streit darüber, wo denn eigentlich der "Mittelpunkt" von Deutschland sei.

Wohin würdest du ihn legen? Begründe deine Entscheidung und erkläre, wie man diesen Mittelpunkt konstruieren kann!

Zusatz: Nimm eine „echte“ Deutschlandkarte und finde den Ort, der sich entsprechend deiner Konstruktion am ehesten Mittelpunkt von Deutschland nennen dürfte!

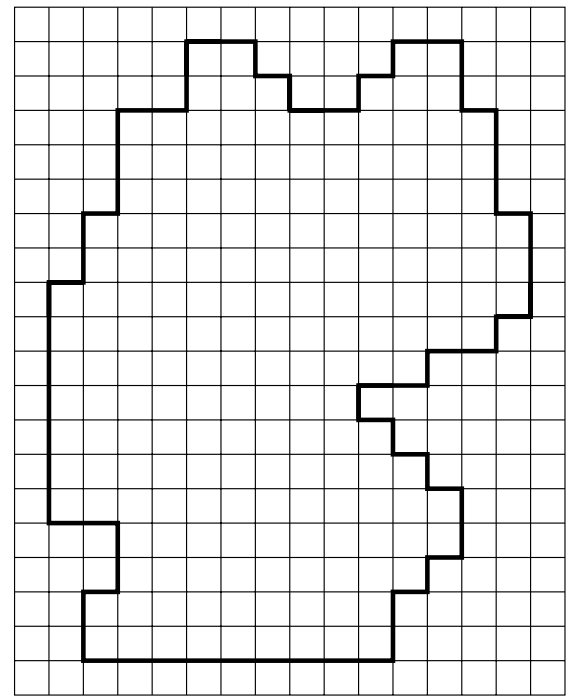




\section{Aufgabenblatt 13}

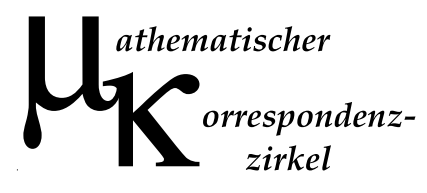

A 13.1 Die Drillinge Anton, Bert und Christoph bekommen von ihrer Oma zum Geburtstag gemeinsam $100 €$. Da sie dies unter sich nicht gerecht aufteilen können, verspricht die Oma, ihnen am nächsten Tag einen weiteren Euro, am darauf folgenden zwei weitere und an jedem weiteren Tag immer einen Euro mehr als am Vortag zu schenken; so lange, bis die drei den Gesamtbetrag unter sich aufteilen können. Wie viele Euro bekommt jeder der Enkel schließlich?

A 13.2 Tante Erna sitzt mit Onkel Heinz beim Kaffeekränzchen. Erna hat vor sich eine Tasse Kaffee stehen, natürlich schwarz, ohne Milch und Zucker. Onkel Heinz trinkt lieber ein Glas Vollmilch, wegen seines Blutdrucks.

Aus seinem fast vollen Glas nimmt er ein Löffelchen Milch, schüttet es in Tante Ernas Tasse und rührt kräftig um. Die verärgerte Tante nimmt ihm den Löffel ab und schüttet ihrerseits einen Löffel voll mit dem Gemisch aus ihrer Tasse in Onkel Heinz' Glas.

Jetzt haben also beide wieder die gleiche Menge Getränk wie am Anfang in ihren Gefäßen. Aber: Hat Onkel Heinz nun mehr Kaffee in seinem Glas als Tante Erna Milch in ihrer Tasse oder ist es umgekehrt?

Zusatz: Wenn nun Onkel Heinz und Tante Emma dieselbe Prozedur noch einmal wiederholen, also ein Löffelchen aus dem Glas in die Tasse, umrühren und danach umgekehrt, wie lautet dann die Antwort?

A 13.3 Gegeben sei ein rechtwinkliges Dreieck $A B C$ mit rechtem Winkel bei $C$. Der Radius des Umkreises des Dreiecks $A B C$ habe die Länge $R$, der Radius des Inkreises habe die Länge $r$.

Beweise, dass dann stets die Beziehung

$$
\overline{A C}+\overline{B C}=2(R+r)
$$

gilt.

A 13.4 Peter Pfiffig will sich einen Wecker bauen und dazu eine Balkenwaage (die in Ruheposition im Gleichgewicht ist) und einige Kerzen benutzen. Die Kerzen will er auf den Waagschaalen positionieren und sie gleichzeitig beim Zubettgehen um 22.00 Uhr anzünden, worauf diese mit konstanter Geschwindigkeit abbrennen und Masse verlieren. Die Kerzen sind dabei so beschaffen, dass sie in einer Stunde um genau einen Zentimeter abbrennen. An der Waage ist ein Kontakt so angebracht, dass immer bei Gleichgewicht eine Glocke läutet, die Peter wecken soll. 
Aufgabenblatt 13

Wie muss Peter die Kerzen verteilen, wie viele müssen es sein und welche Länge müssen sie haben, damit er einmal um 6.00 Uhr und zur Sicherheit nochmals um 6.20 Uhr und um 6.40 Uhr geweckt wird?

Hinweis: Die Kerzen dürfen dabei auch verschieden lang sein! 


\title{
Aufgabenblatt 14
}

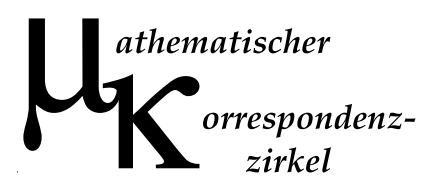

\begin{abstract}
A 14.1 Laura bastelt gerne außergewöhnliche Mikadospiele. So ein Mikadospiel besteht aus 37 Stäbchen, die jedoch nicht alle dieselbe Länge haben müssen, insbesondere können die Längen auch sehr klein oder sehr, sehr groß sein. (Außerdem kann jedes Spiel anders aussehen!) Laura behauptet nun: „Egal wie ich die Stäbe zurechtschneide, in jedem Spiel kann ich drei finden, die ich zu einem Dreieck zusammenlegen kann."

Kannst du Lauras Aussage bestätigen, oder irrt sie sich da?
\end{abstract}

A 14.2 Herr Schluckspecht ist wieder auf Kneipentour. Nach einem feucht-fröhlichen Beginn im Kummerstübchen macht er sich (mit konstanter Geschwindigkeit) auf den Weg zum Dorfkrug. Zur gleichen Zeit bricht Herr Suffkopp vom Dorfkrug in Richtung Kummerstübchen auf. Als sie sich treffen, sagt Herr Schluckspecht (wahrheitsgemäß): „Ich bin $200 \mathrm{~m}$ weiter gegangen als du!“

Es folgt eine engagierte Diskussion ...

Danach gehen sie verstimmt weiter, wegen leichter Blessuren aber jeweils mit halber Geschwindigkeit. Herr Schluckspecht braucht noch 8 min bis zum Dorfkrug, Herr Suffkopp noch 18 min bis zum Kummerstübchen.

Wie weit sind die beiden Kneipen voneinander entfernt?

A 14.3 Für die ersten natürlichen Zahlen gilt:

$$
\begin{aligned}
& 0=-1^{2}-2^{2}+3^{2}-4^{2}+5^{2}+6^{2}-7^{2} \\
& 1=+1^{2} \\
& 2=-1^{2}-2^{2}-3^{2}+4^{2} \\
& 3=-1^{2}+2^{2} \\
& 4=-1^{2}-2^{2}+3^{2}
\end{aligned}
$$

Lassen sich alle natürlichen Zahlen in der Form $\pm 1^{2} \pm 2^{2} \pm 3^{2} \pm \ldots \pm m^{2}$ darstellen?

A 14.4 Wähle dir zwei beliebige natürliche Zahlen $m$ und $n$. Zeichne dann auf kariertes Papier ein Rechteck, das genau $m$ Kästchen lang und $n$ Kästchen breit ist. Jetzt versuche einen geschlossenen Weg entlang der Kästchenkanten ohne Überschneidungen zu finden, der jeden Gitterpunkt im Inneren und auf dem Rand des Rechtecks genau einmal berührt. In der Abbildung ist ein Beispielweg für ein Rechteck mit $m=8$ und $n=5$ dargestellt.

Wenn du das für verschiedene Werte $m$ und $n$ mehrfach probiert hast, wirst du vielleicht feststellen, dass es manchmal mehrere Möglichkeiten gibt, einen solchen Weg 


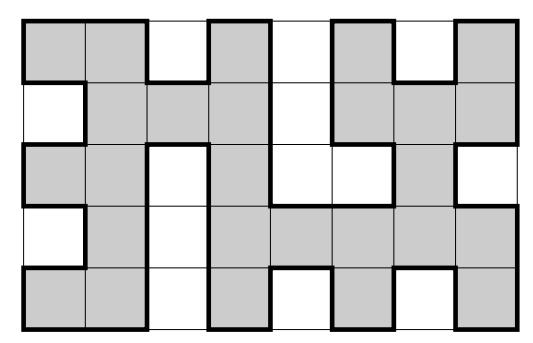

zu finden, manchmal aber auch gar keine. Untersuche, wie das mit der Wahl von $m$ und $n$ zusammenhängt, und versuche, deine Vermutung zu beweisen.

Falls es für festes $m$ und $n$ einen solchen Streckenzug gibt, dann schließt dieser einen Teil des Rechteckes ein (da der Streckenzug ja geschlossen ist). Untersuche, wie sich die Anzahl der Kästchen im Inneren ändert, wenn man bei ein und demselben Rechteck verschiedene Streckenzüge betrachtet. Kannst du deine Vermutung begründen? 


\section{Aufgabenblatt 15}

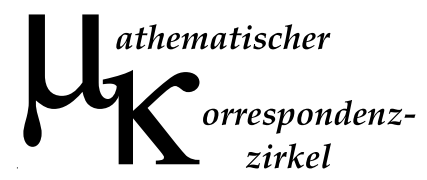

A 15.1 In der Klasse 8a sind mehr Mädchen als Jungen, in der Klasse 8b ist es umgekehrt. Ein Mädchen und ein Junge wechseln von der 8a in die 8b.

Zeige, dass sich dadurch der Anteil der Mädchen in beiden Klassen erhöht!

A 15.2 Jemand addiert alle sechsstelligen Zahlen, die keine der Ziffern 0, 1, 4, 6, 7 oder 9 enthalten. Was ist das Ergebnis?

A 15.3 Drei Freunde wollen das Körbewerfen beim Basketball üben.

Dazu sagt der erste: „Ich wette, dass wenigstens einer von euch beim ersten Versuch keinen Korb wirft."

"Ich wette“, antwortet der zweite, „dass, wenn du beim ersten Mal triffst, du deine Wette gewinnen wirst."

Der dritte sagt daraufhin: „Und ich wette ganz einfach, dass wir alle beim ersten Wurf treffen."

Sie fangen an zu üben. Ist es möglich, dass der zweite und der dritte Freund beide ihre Wette gewonnen oder verloren haben?

A 15.4 Ein Rechteck mit den Seitenlängen $5 \mathrm{~cm}$ und $9 \mathrm{~cm}$ wird in kleinere Rechtecke mit ganzzahligen Seitenlängen (in $\mathrm{cm}$ ) zerlegt.

Bestimme eine Zerlegung mit möglichst vielen Rechtecken, von denen keine zwei deckungsgleich sind. 



\section{Aufgabenblatt 16}

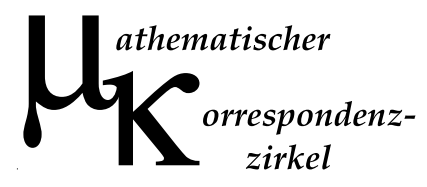

A 16.1 Bei Wintereinbruch haben sich Anton (a), Beate (b) und Christoph (c) als Erste auf das Eis des zugefrorenen Dorfsees gewagt. Sie befinden sich an den in der Abbildung dargestellten Orten auf dem See.

Die Mütter der Kinder (A, B und C) sind zum See geeilt, weil sie in den Nachrichten folgende Meldung gehört haben:

Achten Sie auf Ihre Kinder! Das Eis des Sees ist noch so dünn, dass es bei zweimaligem Betreten einer Stelle in jedem Fall bricht.

Können die Kinder unter diesen Bedingungen auf dem See Eis laufend zu ihren jeweiligen Müttern gelangen, ohne dass eines von ihnen einbricht?

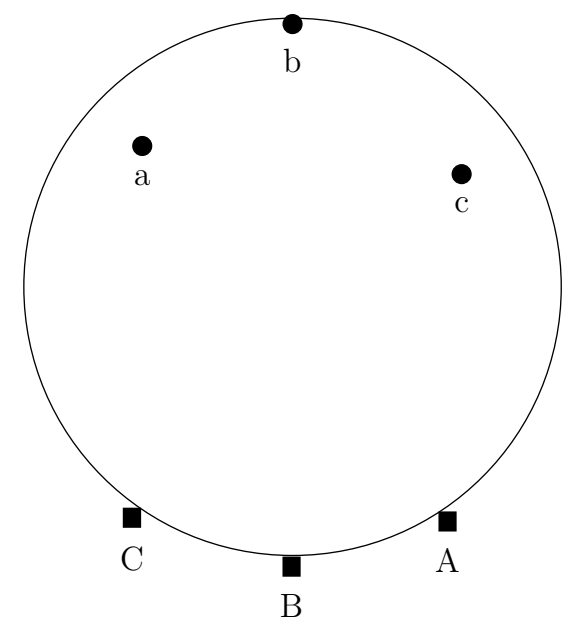

Hinweis: Die Kinder seien zu ihren jetzigen Standorten gelangt, ohne das Eis an einer anderen Stelle zu betreten, zum Beispiel indem sie vom Rand dorthin gesprungen sind.

A 16.2 Ist $p$ eine Primzahl und auch $p+2$ eine Primzahl, so nennt man das Paar $(p, p+2)$ einen Primzahlzwilling.

Man betrachte solche Primzahlzwillinge, bei denen jede der beiden Primzahlen größer als 5 ist. Beweise, dass man dann die Summe der beiden Primzahlen stets als Produkt von wenigstens 4 natürlichen Zahlen, die größer oder gleich 2 sind, schreiben kann!

Beispiel: 107 und 109 sind beide Primzahlen und es gilt: $107+109=216=3 \cdot 3 \cdot 4 \cdot 6$. 
Aufgabenblatt 16

A 16.3 Ein Mann erzählt einem Reporter folgende spannende Geschichte: „... Ich war an diesem Tag zunächst von meiner Hütte aus $10 \mathrm{~km}$ nach Süden gelaufen, als mich ein Sturm überraschte. Sowie dieser abflaute, entschied ich mich, nun weiter in Richtung Osten zu laufen. Dies tat ich $10 \mathrm{~km}$ weit, als plötzlich ein riesiges Tier ganz in meiner Nähe auftauchte - zum Glück hatte ich meine Tarnjacke an, so dass mich das Tier nicht bemerkte. Nach diesem Schreck machte ich mich auf den Weg nach Norden und nach einer $10 \mathrm{~km}$ langen Wanderung in dieser Richtung erreichte ich wieder meine Hütte. ..."

Versuche, alle Orte auf der Erde zu bestimmen, an denen die Hütte des Abenteurers entsprechend dieser Geschichte stehen kann (es gibt mehrere!), und bestimme die Farbe der Jacke!

A 16.4 Weihnachten steht vor der Tür und somit auch die jedes Jahr kurz nach Weihnachten stattfindende Rentieriade. Alle Weihnachtsmänner messen sich dabei im Rentierschlittenrennen auf einer Stadionrunde.

Die Rentiere - von Natur aus faule und gefräßige Tiere - haben hierbei immer einen Futterkorb vor dem Maul, und sie laufen nur, solange darin noch Futter ist. Entlang der Stadionrunde sind in unregelmäßigen Abständen 17 Futterstationen eingerichtet, an denen die Futterkörbe nachgefüllt werden können. Auf diese Stationen hat die Jury (die Osterhasen) Futter verteilt, das insgesamt genau für eine Stadionrunde reicht. Die Menge an jeder Station legt sie für jeden Teilnehmer beliebig neu fest, und zwar insbesondere unabhängig davon, wie weit die jeweils nächste Station entfernt ist.

Jedem Teilnehmer wird sein Schlitten mit leerem Futterkorb an einem Punkt seiner Wahl bereitgestellt. Kann er stets - egal wie das Futter verteilt ist - eine der Stationen als Startpunkt so aussuchen, dass er mit seinem Schlitten die volle Stadionrunde schafft? 


\section{Aufgabenblatt 17}

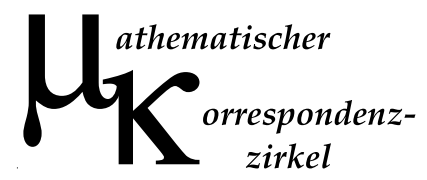

A 17.1 Frau Porta hat in ihren 1 Meter breiten Flur zwei Türen eingebaut. Die Türen sind an gegenüberliegenden Ecken befestigt und nehmen jeweils die volle Breite ein (vergleiche Skizze). Glücklicherweise blockieren sich die beiden Türen nicht gegenseitig.

Wie lang ist ihr Flur mindestens?

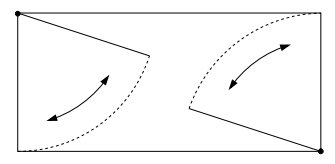

A 17.2 Ein handelsüblicher Dominostein besteht aus zwei Hälften, auf denen jeweils $0,1, \ldots, 5$ oder 6 Punkte markiert sind. Zu einem Spiel gehören 28 Steine, wobei jede Kombination von Punktezahlen genau einmal auftritt. Kann man mit allen Dominosteinen eine Kette so basteln, dass aufeinander stoßende Steinhälften stets die gleichen Punktezahlen tragen?

Gibt es eine solche Kette, bei der an den freien Enden die Punktezahlen jeweils 1 sind? Kann man erreichen, dass an einem Ende eine 1 und am anderen eine 6 liegt?

A 17.3 Kann man die folgende Figur durch einen Schnitt entlang der Kästchenkanten in zwei kongruente Teile zerlegen, bei denen sich Ecke $A$ und Ecke $B$ entsprechen?

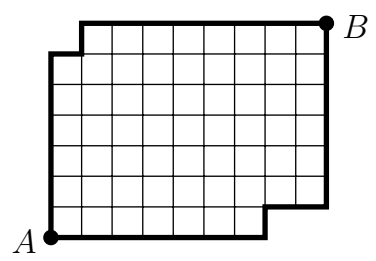

A 17.4 Jemand rechnet das Produkt $42 !=42 \cdot 41 \cdot \ldots \cdot 2 \cdot 1$ aus und schreibt das Ergebnis auf einen Zettel. Nach einigen Tagen stellt er fest, dass der Zettel nass geworden ist und drei Ziffern nicht mehr lesbar sind:

$$
42 !=140500611775287989854 \text { 苜14260624:511569936384\$00000000 }
$$

Kann man die fehlenden Ziffern rekonstruieren, ohne das Produkt erneut ausrechnen zu müssen? 



\section{Aufgabenblatt 18}

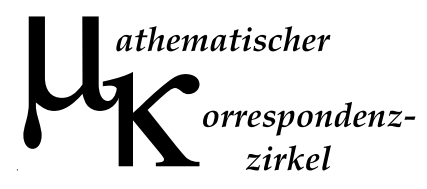

A 18.1 Jemand bildet aus den Ziffern 1,2,3,4,5 und 6 sechsstellige natürliche Zahlen, wobei jede der Ziffern genau einmal benutzt wird.

Kann unter diesen Zahlen eine Quadratzahl sein?

A 18.2 Ein Holzwürfel hat die Kantenlänge $30 \mathrm{~cm}$ und soll in 27 kleinere Würfel mit der Kantenlänge $10 \mathrm{~cm}$ zersägt werden. Beim Zersägen wird in jedem Schritt ein gerader Schnitt ausgeführt, wobei aber eventuell auch mehrere vorhandene Teile übereinandergestapelt und somit gleichzeitig durchgesägt werden können.

Wie viele Sägeschnitte muss man mindestens machen, um den großen Würfel vollständig in die kleinen zu zerlegen?

A 18.3 Ingolf und Anke wollen am Strand spielen und schlagen hierzu ihre Lager an den in der Lageskizze angegebenen Orten auf. Während Anke eine Sandburg bauen will, hat Ingolf nur deren Zerstörung im Sinn. Es geschieht Folgendes:

Anke baut zunächst ihre Sandburg irgendwo am Strand und begibt sich dann wieder zu ihrem Lager. Ingolf versucht nun, mit seinem Fußball die Sandburg von seinem Standort aus zu treffen. Der Ball bewegt sich dabei auf einer geraden Linie mit einer konstanten Geschwindigkeit von $12 \frac{\mathrm{m}}{\mathrm{s}}$. Sowie ihn Ingolf auf seine zerstörerische Reise geschickt hat, rennt Anke mit einer konstanten Geschwindigkeit von $4 \frac{\mathrm{m}}{\mathrm{s}}$ los, um den Ball aufzuhalten.

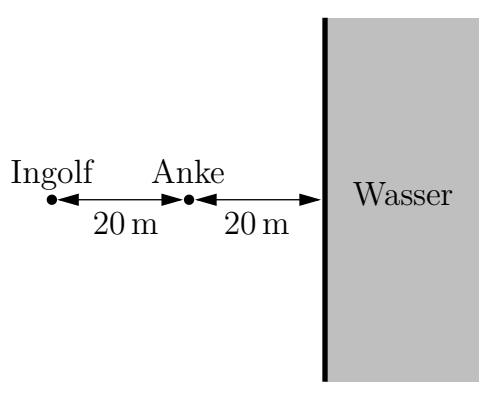

Versuche zunächst, einige Orte am Strand zu finden, an denen Anke bedenkenlos ihre Sandburg bauen kann, ohne deren Zerstörung befürchten zu müssen (weil sie den Ball rechtzeitig abfangen kann)!

Welches ist das größte Gebiet, das Anke auf die beschriebene Weise von ihrem Platz aus verteidigen kann? 
Aufgabenblatt 18

A 18.4 Asterix und Obelix werfen eine Münze (die neue Ein-Euro-Sesterze) und schreiben auf, in welcher Reihenfolge Adler $(A)$ oder Zahl $(Z)$ oben liegen. Asterix hat sich die Dreierfolge $A A Z$ ausgesucht und Obelix die Folge $Z A A$. Sie werfen die Münze, bis eine der beiden Dreierfolgen auftaucht. Es gewinnt, wessen Folge erschienen ist.

a) Wirf selbst ein paar Mal eine Münze und schau nach, wer öfter gewinnt.

b) Berechne die Wahrscheinlichkeit, mit der Asterix gewinnt.

c) Wie ändern sich die Chancen, wenn Obelix als Folge $A Z A$ wählt? 


\section{Aufgabenblatt 19}

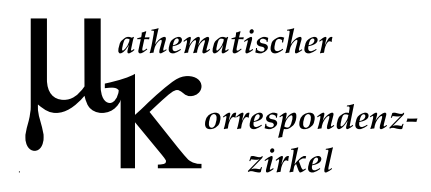

\section{A 19.1}

a) In dem „Buch der Wahrheit“ stehen merkwürdige Dinge:

Auf der ersten Seite steht: „In diesem Buch steht genau eine falsche Aussage.“ Auf der zweiten Seite steht: „In diesem Buch stehen genau zwei falsche Aussagen."

Und so weiter, bis schließlich auf Seite 2002, der letzten Seite, steht: „In diesem Buch stehen genau 2002 falsche Aussagen."

Wie viele falsche Aussagen stehen tatsächlich in diesem Buch, und wo stehen sie gegebenenfalls?

b) Das „Buch der Lügen“ ist etwas allgemeiner gefasst:

Auf der ersten Seite steht: „In diesem Buch steht mindestens eine falsche Aussage."

Auf der zweiten Seite steht: „In diesem Buch stehen mindestens zwei falsche Aussagen."

Und so weiter, bis schließlich auf Seite 2002, ebenfalls der letzten Seite, steht: "In diesem Buch stehen mindestens 2002 falsche Aussagen."

Wie viele falsche Aussagen stehen nun in diesem Buch, und wo stehen sie gegebenenfalls?

A 19.2 Wie viele Möglichkeiten gibt es, vier Türme so auf ein Schachbrett zu stellen, dass keiner einen anderen bedroht?

A 19.3 Um ein konvexes Polygon, das einen Umfang $U$ hat, wird ein Rahmen gezeichnet, dessen Außenlinien in einem Abstand $b$ parallel zu den Polygonseiten verlaufen (vgl. Zeichnung).

Beweise, dass die Fläche des Rahmens größer als $b(U+\pi b)$ ist.

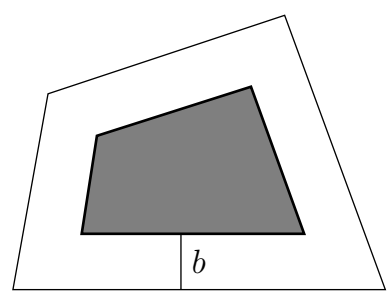

Hinweis: Ein konvexes Polygon ist ein Vieleck, in dem alle Innenwinkel kleiner als $180^{\circ}$ sind. 
Aufgabenblatt 19

A 19.4 Diesmal eine Fortsetzungsaufgabe! (Keine Sorge, dazu muss man die Aufgabe vom letzten Zettel nicht gelöst haben.)

Zur Erinnerung: Asterix und Obelix spielen ein Spiel. Dabei werfen sie eine Münze und schreiben auf, in welcher Reihenfolge Adler $(A)$ oder Zahl $(Z)$ oben liegen. Jeder hat sich eine Dreierfolge ausgesucht, und das Spiel wird beendet, sobald in der (bis dahin evtl. recht langen) Folge der Ergebnisse eine der beiden Dreierfolgen auftaucht. Es gewinnt natürlich, wessen Folge erschienen ist.

a) Man hätte zunächst denken mögen, dass keiner der beiden einen Vorteil hat, weil ja jede Dreierfolge mit gleicher Wahrscheinlichkeit einmal geworfen wird. Warum kann man dieses Argument hier eigentlich nicht anwenden?

b) Auf dem letzten Zettel wurde errechnet, dass $Z A A$ gegen $A A Z$ mit $3 / 4$ zu $1 / 4$ und $A A Z$ gegen $A Z A$ mit $2 / 3$ zu $1 / 3$ gewinnt. Gewinnt also auch im Mittel $Z A A$ gegen $A Z A$ ? 


\section{Aufgabenblatt 20}

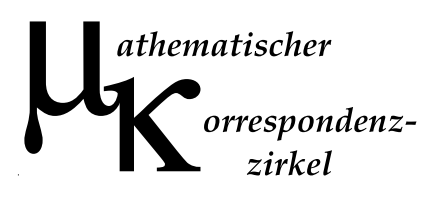

A 20.1 Gegeben sei folgendes Gleissystem (ein Kreis mit einem Abstellgleis):

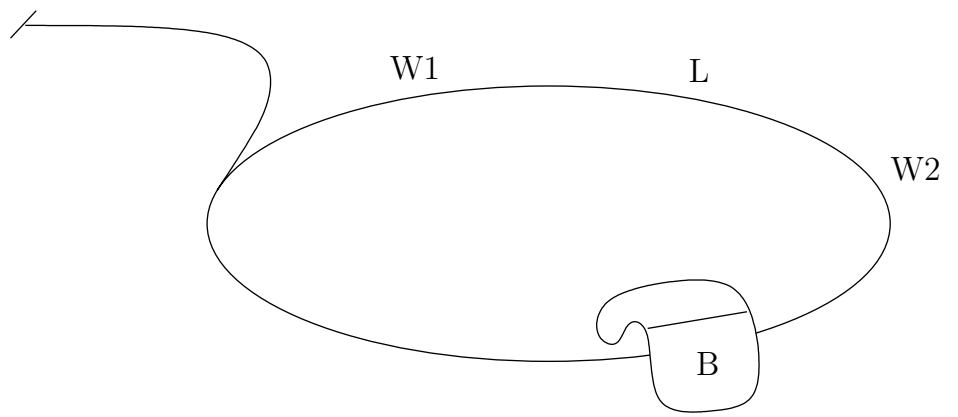

Auf dem Kreis stehen eine Lok (L) und zwei Wagen (W1 und W2). Aufgabe ist es, die beiden Wagen durch Rangieren zu vertauschen. Die Lok darf dabei schieben und ziehen. Durch die Brücke (B) passt aber nur die Lok und dort darf sie auch höchstens zweimal durch. Ganz wichtig: Zum Schluss muss die Lok wieder am Ausgangspunkt stehen.

A 20.2 Wie kann man aus den Ziffern 1, 5, 6 und 7 die Zahl 21 darstellen? Jede Ziffer darf und muss genau einmal benutzt werden, allerdings in beliebiger Reihenfolge. Außerdem dürfen jeweils beliebig häufig die vier Grundrechenarten (Addition, Subtraktion, Multiplikation und Division) verwendet und Klammern gesetzt werden. Andere Rechenzeichen sind nicht erlaubt.

Beispiel: Die Zahl 22 ließe sich auf diese Weise wie folgt darstellen: $22=7 \cdot(5-1)-6$.

A 20.3 Man nehme eine beliebige vierstellige Zahl, deren Ziffern nicht alle gleich sind. Dann ordne man die Ziffern der Größe nach um, einmal mit der größten und einmal mit der kleinsten beginnend. Die beiden so erhaltenen Zahlen ziehe man voneinander $\mathrm{ab}$.

Beispiel: Beginnt man mit 1990, so erhält man 9910 bzw. 0199, als Differenz ergibt sich $9910-0199=9711$.

Mit der als Differenz erhaltenen Zahl wiederhole man die gleiche Prozedur (gegebenenfalls fülle man vorher die vorderen Stellen mit Nullen auf, wenn die Differenz nicht vierstellig war) usw.

Was passiert nach einigen Wiederholungen? Was ergibt sich bei anderen vierstelligen Startzahlen? Stelle eine Vermutung auf und versuche, diese zu beweisen. 
A 20.4 Nach welchem Algorithmus entstand das folgende Muster?

(Quelle: http://www.math 4 u.de)

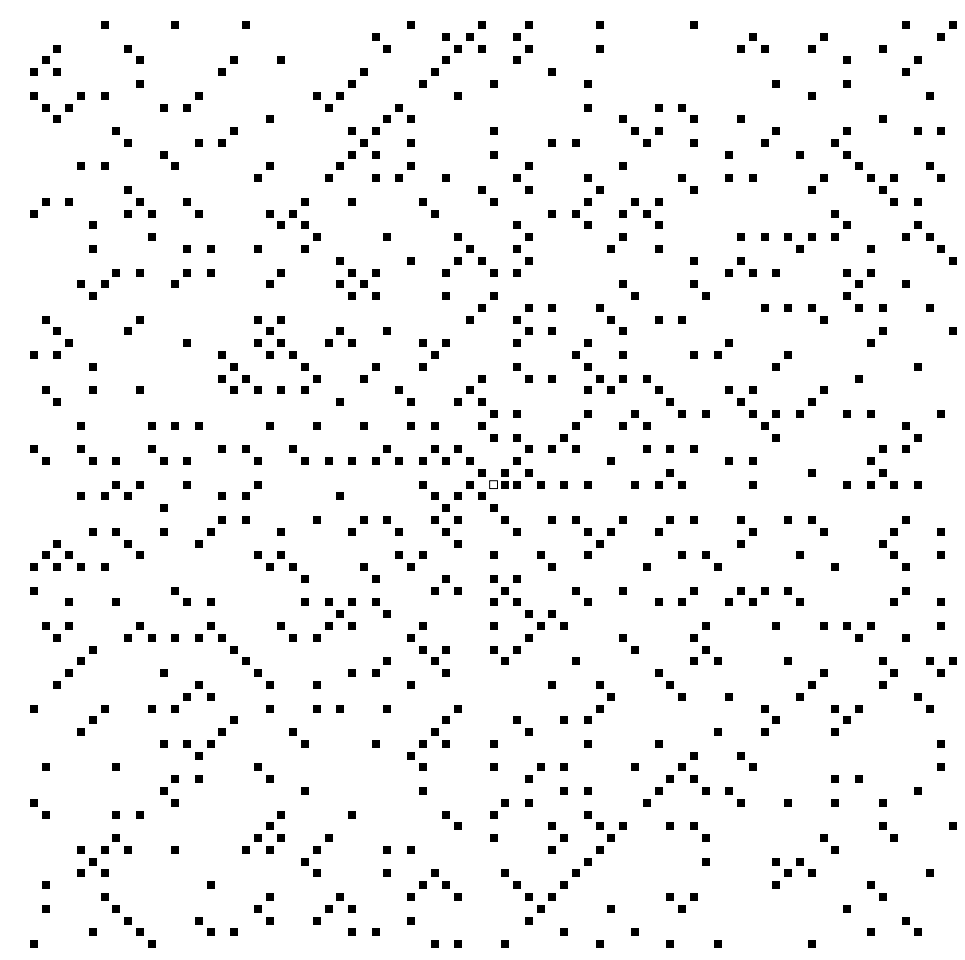

Zusatz: Wie lassen sich die weißen, ungefähr von der Mitte ausgehenden Streifen erklären? Kannst du erklären, wie die schwarzen Diagonalen entstehen? 


\section{Aufgabenblatt 21}

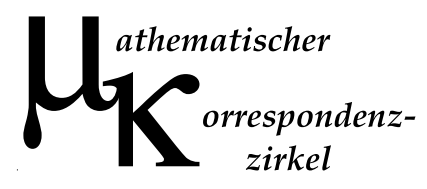

A 21.1 Aus der Folge $1, \frac{1}{2}, \frac{1}{3}, \ldots$ der Kehrwerte der natürlichen Zahlen kann man leicht eine dreigliedrige arithmetische Teilfolge auswählen: $\frac{1}{2}, \frac{1}{3}, \frac{1}{6}$. Ebenso gibt es arithmetische Teilfolgen mit 4 Gliedern, z. B. $\frac{1}{3}, \frac{1}{4}, \frac{1}{6}, \frac{1}{12}$.

a) Finde arithmetische Teilfolgen mit 5 Gliedern und mit 6 Gliedern.

b) Ist es möglich, für jede natürliche Zahl $k$ eine arithmetische Teilfolge mit $k$ Gliedern zu finden?

Hinweis: Eine Folge $a_{1}, a_{2}, \ldots, a_{k}$ heißt arithmetisch, wenn die Differenz aufeinander folgender Glieder konstant ist, d. h. wenn $a_{1}-a_{2}=a_{2}-a_{3}=\ldots=a_{k-1}-a_{k}$ ist.

A 21.2 Aus einem Quadrat mit Seitenlänge $a$ werden drei deckungsgleiche gleichschenklige Dreiecke, so wie es die Skizze zeigt, herausgeschnitten.

Welchen Flächeninhalt hat das Reststück?

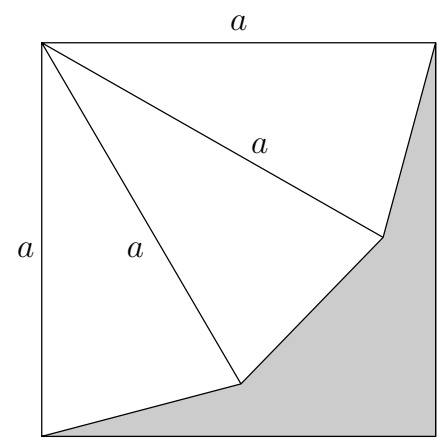

Hinweis: Gesucht ist - der Schönheit wegen - eine Lösung, die ohne Benutzung der Winkelfunktionen (sin, cos, tan, cot) auskommt.

A 21.3 Paul fährt in den Urlaub nach Möwenland. Er weiß, dass es dort fünf Städte gibt, die durch ein Eisenbahnnetz verbunden sind, und zwar durch genau vier Strecken, die jeweils eine der Städte mit einer anderen verbinden. (Es kann auch sein, dass sich zwei Strecken kreuzen, weil dort eine Brücke errichtet wurde Möwenland hat bekanntlich einige Berge.)

Wie viel verschiedene solcher Eisenbahnnetze kann es geben? 
Aufgabenblatt 21

A 21.4 Unser Grill ist zu klein: Auf dem Rost haben maximal zwei Steaks gleichzeitig Platz, wir wollen aber insgesamt drei Steaks grillen - und zwar in möglichst kurzer Zeit!

Für die einzelnen Teilschritte haben wir folgende Zeiten ermittelt:

Ein Steak braucht genau zweieinhalb Minuten, um von einer Seite gar zu werden, und noch einmal zweieinhalb Minuten für die andere Seite. Wir benötigen 15 Sekunden, um ein Steak auf den Grill zu legen oder vom Grill zu nehmen, genauso lange benötigt man auch, um ein Steak direkt auf dem Grill (also ohne Herunternehmen) zu wenden. Und ganz wichtig: Die Steaks müssen noch einseitig mit einer speziellen Sauce bestrichen werden, dazu müssen sie aber mindestens von einer Seite vollständig gegrillt sein. Das Bestreichen eines Steaks dauert genau eine Minute, kann allerdings nicht ausgeführt werden, solange das Steak auf dem Grill liegt (schließlich ist es über dem Grill ziemlich heiß). Man darf es aber anschließend wieder auf den Grill legen, wenn es noch nicht vollständig von der anderen Seite gegrillt ist. Es ist nicht möglich, zwei Steaks gleichzeitig zu bestreichen.

$\mathrm{Zu}$ Beginn ist der Grill schon heiß. Wie lange würdest du brauchen, bis alle drei Steaks einseitig mit Sauce bestrichen und beidseitig gegrillt sind? 


\section{Aufgabenblatt 22}

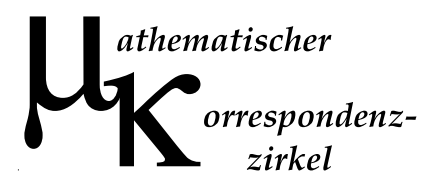

A 22.1 Leonard zeichnet mehrere gerade Linien auf ein Blatt Papier, jeweils von einer Kante des Blattes zu einer anderen. Bei näherem Hinschauen sieht er, dass er die Linien so gezeichnet hat, dass jede Linie jede andere schneidet und keine drei Linien sich in einem Punkt schneiden. Er zählt nun nach, dass auf diese Weise das Blatt in 22 Teile aufgeteilt wurde.

a) Wie viele Linien hat Leonard gezeichnet?

b) Erhält man immer 22 Teile, wenn man genauso viele Linien wie Leonard zeichnet, die sich auf dieselbe Weise schneiden?

c) Wie lautet die Antwort bei mehr oder weniger Linien? Versuche eine Formel anzugeben, um die Anzahl der Teile zu berechnen.

A 22.2 Für Lenas Glückszahl $x$ gilt

$$
3=\sqrt{x+\sqrt{x+\sqrt{x+\ldots}}}
$$

Welche Zahl bringt Lena Glück?

A 22.3 Bauer Hauser hat 4 Söhne, unter denen er sein großes Feld, das die Form eines konvexen Vierecks hat, aufteilen möchte. Hierzu verwendet er folgende Methode (Bezeichnungen wie in der Skizze): Er misst die Mitten $M_{a}$ bis $M_{d}$ der Seiten und die Mitte $M$ der Diagonale BD ab. Der erste Sohn bekommt dann das Viereck $A M_{a} M M_{d}$, der zweite das Viereck $B M_{b} M M_{a}$, der dritte $C M_{c} M M_{b}$ und der vierte $D M_{d} M M_{c}$.

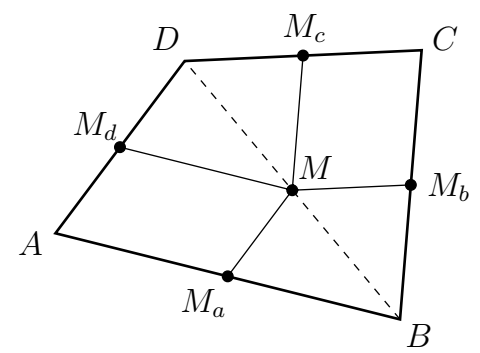

Ist diese Aufteilung gerecht, bzw. unter welchen Umständen wird welcher Sohn bevorteilt? 
Aufgabenblatt 22

A 22.4 Monika rechnet etwas mit Zahlen herum. Sie geht dabei folgendermaßen vor:

Sie beginnt mit vier natürlichen Zahlen (alle größer als 0). Sie bildet alle möglichen Paare dieser Zahlen (erste und zweite Zahl, erste und dritte Zahl, ..., vorletzte und letzte Zahl). Falls die Zahlen eines Paares verschieden sind, subtrahiert sie die kleinere von der größeren. Alle erhaltenen Differenzen schreibt sie auf ein neues Blatt Papier.

Mit den Zahlen dieses neuen Blattes geht sie nun genauso vor: Paare bilden, Differenzen ausrechnen, Differenzen auf ein neues Blatt schreiben.

Dies macht sie so lange, bis auf einem Blatt entweder nur noch genau eine Zahl oder gar keine Zahl mehr steht.

a) Zeige, dass Monika nur endlich viele Blatt Papier verwenden muss.

b) Gibt es Anfangszahlen, bei denen am Schluss eine Zahl übrig bleibt, oder endet Monikas Prozedur immer mit einem leeren Blatt?

Beispiel für Monikas Rechnung:

Sie beginnt mit den Zahlen 2, 5, 5 und 8 und erhält als Differenzen: $5-2=3,5-2=$ $3,8-2=6,8-5=3$ und $8-5=3$ ( 5 und 5 sind nicht verschieden; das Paar $(5,5)$ wird also nicht weiter betrachtet). Auf dem neuen Blatt Papier stehen also die Zahlen 3,3,3,3 und 6 .

Nach erneuter Ausführung der Prozedur stehen auf dem dritten Blatt die Zahlen $3,3,3$ und 3 .

In allen sechs Paaren (jeweils bestehend aus 3 und 3) dieser Zahlen sind die beiden Zahlen gleich. Somit bleibt das nächste Blatt leer und Monika kann nicht mehr weiterrechnen. 


\section{Aufgabenblatt 23}

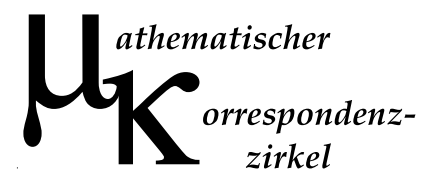

A 23.1 Auf einer Geburtstagsparty sind 97 Gäste eingeladen. Manche davon kennen sich, andere wiederum nicht (wie das meistens der Fall ist).

Zeige, dass es unter diesen Gästen (mindestens) zwei gibt, die die gleiche Zahl von Gästen kennen.

A 23.2 Marlene entdeckt eine hübsche Eigenschaft der Zahl 2002:

2002 liegt zwischen den Quadratzahlen $1936=44^{2}$ und $2025=45^{2}$, d.h. $1936 \leq 2002<2025$. Die Zahl $2002-(2002-1936)(2025-2002)=484=22^{2}$ ist wieder eine Quadratzahl.

Welche natürlichen Zahlen haben die gleiche hübsche Eigenschaft?

A 23.3 Zur Geburt seines Sohnes pflanzt Freiherr vom Kiesel auf seinem Anwesen eine Eiche, und zwar an einer Stelle, die von der Nordwestecke der großen rechteckigen Rasenfläche 210 Meter und von der Südostecke 180 Meter entfernt ist. Von der Nordostecke hat der Baum einen Abstand von 60 Metern.

Wie weit ist es von der Eiche bis zur vierten Ecke des Rasens?

A 23.4 Jemand schreibt in einem ersten Schritt die Zahl 1 auf ein Blatt Papier und fügt dann schrittweise immer die Ziffern der nächsten natürlichen Zahl an. Nach 11 Schritten steht auf dem Papier also die Zahl 1234567891011. Wie oft während der ersten 99 Schritte steht auf dem Papier eine durch 11 teilbare Zahl? 



\section{Aufgabenblatt 24}

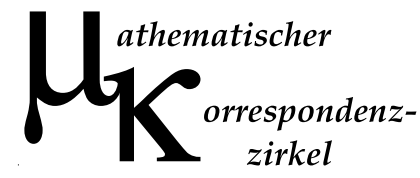

A 24.1 Man beweise, dass man ein Quadrat für jede Zahl $n \geq 6$ in genau $n$ kleinere Quadrate zerlegen kann!

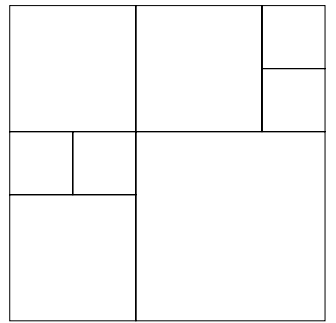

Eine Zerlegung für $n=8$

A 24.2 Kurz vor Weihnachten plant der Weihnachtsmann die Route, auf der er am Weihnachtsabend mit seinem von Rentier Rudolf gezogenen Schlitten einige der 64 Häuser des Städtchens Schneeberg besuchen will. Er fährt entlang des Straßennetzes auf gerader Linie von Haus zu Haus und weiß aus der Erfahrung der vergangenen Jahre, dass es, je leichter der Schlitten wird, immer schwerer wird, den Rentierschlitten mit dem immer enthusiastischer werdenden Rudolf abzubremsen. Deswegen muss er die Route so planen, dass der Weg vom aktuellen zum nächsten Haus immer länger ist als der Weg vom vorherigen Haus zum aktuellen.

Wie viele verschiedene Häuser kann der Weihnachtsmann auf diese Weise höchstens besuchen und wie lang ist sein Weg dabei maximal?

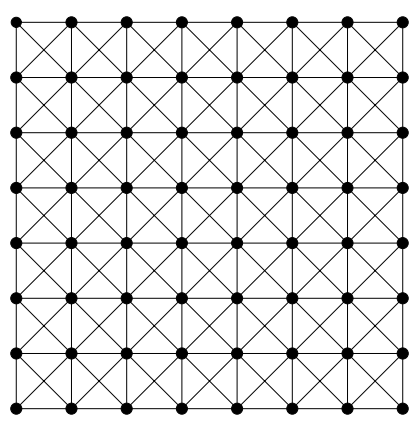

Das Straßennetz in Schneeberg (die Punkte stellen die Häuser dar) 
A 24.3 Unten stehendes Bild zeigt ein multiplikativ-magisches Quadrat. Man sieht, dass die Produkte der drei Zahlen in jeder Zeile, jeder Spalte und den beiden Diagonalen gleich sind, und zwar 64 .

\begin{tabular}{|l|l|l|}
\hline 8 & 4 & 2 \\
\hline 1 & 4 & 16 \\
\hline 8 & 4 & 2 \\
\hline
\end{tabular}

Diese magische Konstante 64 ist interessanterweise eine Kubikzahl, also $64=4^{3}$. Finde weitere multiplikativ-magische Quadrate mit ganzzahligen Einträgen und bestimme jeweils die magische Konstante!

Versuche zu beweisen, dass diese magische Konstante immer eine Kubikzahl ist!

A 24.4 Wir nennen ein Rechteck, dessen Seiten $a$ und $b$ im Verhältnis $a: b=(n+1)^{2}: n^{2}$ stehen, wobei $n$ eine beliebige natürliche Zahl ist, ein ZerlegeRechteck.

Zeige, dass man jedes Zerlege-Rechteck in zwei Teile zerschneiden kann, die sich zu einem Quadrat zusammenfügen lassen. 


\section{Aufgabenblatt 25}

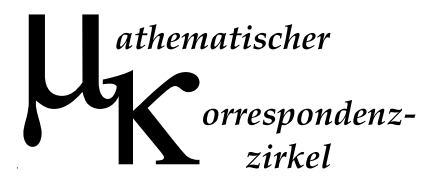

A 25.1 Ein Bleistift, ein Radiergummi und ein Spitzer kosten zusammen genau einen Euro. Außerdem kostet ein Spitzer mehr als zwei Bleistifte, drei Bleistifte kosten mehr als vier Radiergummi und drei Radiergummi kosten mehr als ein Spitzer. Wie viel kostet ein Bleistift, wie viel ein Radiergummi und wie viel ein Spitzer?

Hinweis: Alle Preise sind natürlich ganzzahlige Cent-Beträge.

A 25.2 Neunundzwanzig Bowlingkugeln rollen in einer langen Röhre hintereinander (nicht notwendig in gleichem Abstand) mit konstanter Geschwindigkeit von links nach rechts. Ihnen rollen weitere neunundzwanzig Bowlingkugeln mit derselben Geschwindigkeit von rechts nach links entgegen.

Sobald zwei Bowlingkugeln zusammenstoßen, macht es laut „Pling“ und die beiden Kugeln rollen in entgegengesetzter Richtung mit derselben Geschwindigkeit weiter. Wie viele „Plings" kann ein aufmerksamer Zuhörer registrieren, wenn keine zwei „Plings" gleichzeitig erklingen?

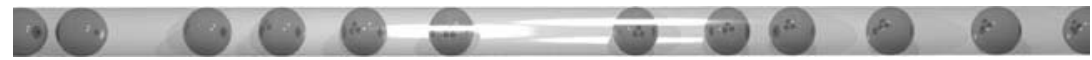

A 25.3 Ein rechtwinkliges Dreieck aus Papier wird entlang einer Geraden so gefaltet, dass eine Ecke des Dreiecks auf der Ecke mit dem rechten Winkel zu liegen kommt. Der Umriss der so entstandenen Figur ist dann ein Viereck. In welchem Verhältnis schneiden sich die Diagonalen dieses Vierecks?

A 25.4 Finde alle fünfstelligen natürlichen Zahlen $n$ mit der Eigenschaft, dass die vierstellige Zahl, die aus $n$ durch Streichen der mittleren Ziffer entsteht, ein Teiler von $n$ ist. 

Teil II

Lösungen 



\section{Lösungen zu Aufgabenblatt 1}

L 1.1 Behauptung: Herr Schulze kommt unter den gegebenen Voraussetzungen genau 10 Minuten früher an als gewöhnlich!

Erster Beweis (rechnerisch): In der ersten Situation kommen die beiden $20 \mathrm{Mi}-$ nuten früher an, also hat der Chauffeur auf jedem seiner beiden Fahrwege (hin und zurück) genau 10 Minuten gespart. Insbesondere trifft er Herrn Schulze schon um 16.50 Uhr. Herr Schulze ist also in den 50 Minuten ab 16.00 Uhr genau den Weg gelaufen, den der Chauffeur in 10 Minuten gefahren wäre. Daraus folgt aber, dass der Chauffeur 5-mal so schnell ist wie Herr Schulze.

In der zweiten Situation, in der Herr Schulze 30 Minuten früher ankommt, treffen sich die beiden $t$ Minuten vor 17.00 Uhr irgendwo zwischen Bahnhof und zu Hause. Herr Schulze läuft also 30-t Minuten. Da er 5-mal so langsam ist wie der Chauffeur, gilt

$$
30-t=5 t \Rightarrow t=5
$$

Der Chauffeur spart auf jedem Weg 5 Minuten, Herr Schulze kommt also 10 Minuten früher an.

Zweiter Beweis (grafisch): Man kann sich die Bewegungen von Herrn Schulze und seinem Chauffeur in einem sogenannten Weltiniendiagramm veranschaulichen. Dieses ist ein Koordinatensystem, auf dessen $x$-Achse die Zeit und auf dessen $y$ Achse der Ort eingetragen wird. Für eine Bewegung kann man nun zu jeder Zeit den Ort feststellen und man erhält eine Kurve. Bei Bewegungen mit konstanter Geschwindigkeit sind dies Geraden. Je schneller eine solche Bewegung verläuft, umso steiler verlaufen die Geraden.

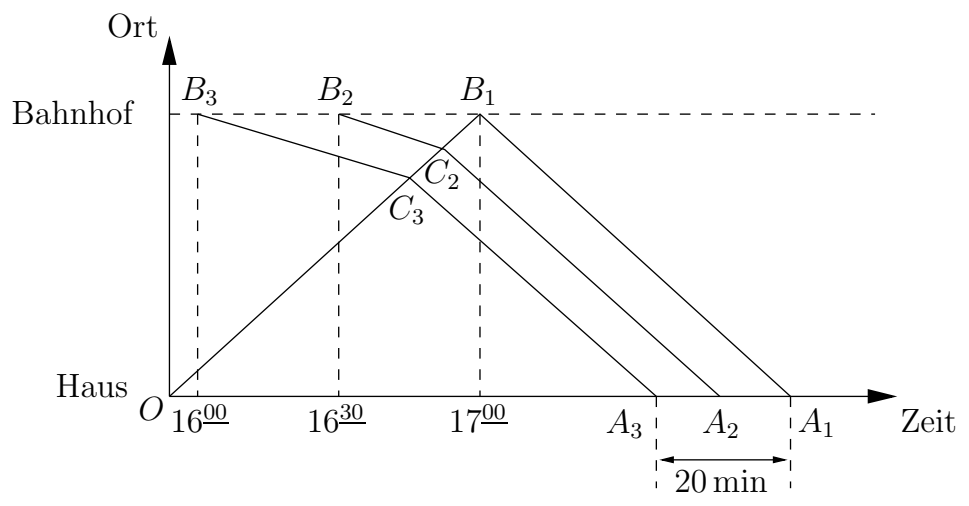

Abbildung 1.1: Weltliniendiagramm 
In Abbildung 1.1 sind die Weltlinien von Herrn Schulze und seinem Chauffeur dargestellt. Im Normalfall fährt der Chauffeur bei $O$ los, trifft am Bahnhof bei $B_{1}$ auf Herrn Schulze und fährt nach $A_{1}$ zurück. In den beiden speziellen Situationen trifft Herr Schulze schon bei $B_{3}$ bzw. $B_{2}$ am Bahnhof ein, läuft los, begegnet dem Chauffeur bei $C_{3}$ bzw. $C_{2}$ und kommt dementsprechend bei $A_{3}$ bzw. $A_{2}$ zu Hause an. Da sich Herr Schulze und sein Chauffeur stets mit der gleichen Geschwindigkeit bewegen, sind ihre Weltlinien parallel, also $B_{3} C_{3} \| B_{2} C_{2}$ und $B_{1} A_{1}\left\|C_{2} A_{2}\right\| C_{3} A_{3}$. Nach Voraussetzung ist nun $\overline{B_{3} B_{2}}=\overline{B_{2} B_{1}}$. Daraus folgt dann nach Strahlensatz auch $\overline{C_{3} C_{2}}=\overline{C_{2} B_{1}}$ und hieraus ebenfalls nach Strahlensatz $\overline{A_{3} A_{2}}=\overline{A_{2} A_{1}}$. Da aber der Strecke $A_{3} A_{1}$ nach Voraussetzung 20 Minuten entsprechen, entspricht der Strecke $A_{2} A_{1}$ genau die Hälfte, also 10 Minuten. Das war zu zeigen.

L 1.2 Die Lösung stammt von Julian aus dem Felix-Klein-Gymnasium in Göttingen:

„Bei jeder Teilung wird das Schokoladenstück, das gerade geteilt wird, in zwei Teile geteilt. Nach einem Schnitt gibt es also genau 1 Schokoladenteil mehr als vorher. Will man also aus dem anfangs vorhandenen Schokoladenteil 16 machen, so ist 15mal eine Erhöhung der Anzahl um 1 nötig, es muss also genau 15-mal geschnitten werden."

Noch eine Anmerkung: Für die Lösung der Aufgabe war es (wie bei vielen Aufgaben) entscheidend, den Aufgabentext genau zu lesen und dabei zu verstehen, was mit einem „Schnitt“ gemeint ist. Dort stand, dass bei einem Schnitt immer ein Teil genommen wird und in zwei Teile zerbrochen wird, deshalb hat man nach jedem Schnitt genau ein Teil mehr. Es ist also völlig egal, wie man schneidet und welche Form die Schnitte haben, man braucht immer genau 15 Schnitte. Es geht nicht mit weniger, aber auch nicht mit mehr.

L 1.3 Zunächst einmal gibt es zwei Möglichkeiten, Palindrome zu erzeugen (siehe Abbildung 1.2).

Variante 1: Man kann sich $m$ Stück unter den Ziffern 0, 1, ., 9 wählen (durchaus auch einige mehrfach oder gar nicht) und diese dann in umgekehrter Reihenfolge noch einmal hinten anfügen. Hierbei sind führende Nullen zu streichen; hat man also etwa für $m=3$ die Ziffern 014 gewählt, so wird daraus 1441, nicht etwa 014410 (=14410, was ja kein Palindrom wäre). Somit entstehen hierbei alle Palindrome mit einer geraden Stellenzahl, die kleiner oder gleich $2 m$ ist.

Variante 2: Man wählt sich $m$ Ziffern und fügt an die $m$-te die $(m-1)$-te, dann die $(m-2)$-te usw. bis zur ersten noch einmal an (wiederum ohne führende Nullen). Hierbei entstehen alle Palindrome mit einer ungeraden Stellenzahl, die kleiner oder gleich $2 m-1$ ist.

Man habe nun eine Zahl $N$ mit $n$ Stellen gegeben und außerdem sei zunächst $n=2 k$ eine gerade Zahl. Nach Variante 2 kann man dann mit $m=k$ alle Palindrome mit ungerader Stellenzahl kleiner oder gleich $N$ erzeugen. Hierzu wählt man sich wie 

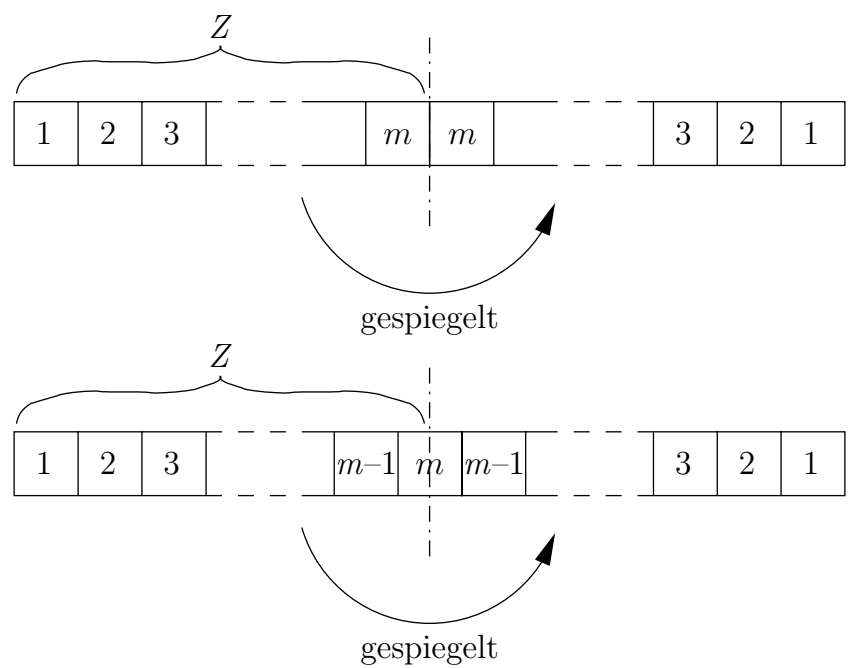

Abbildung 1.2: Erzeugung von Palindromen mit gerader Stellenanzahl (oben) und ungerader Stellenanzahl (unten)

beschrieben $k$-mal eine der 10 Ziffern. Dazu hat man genau

$$
\underbrace{10 \cdot 10 \cdot \ldots \cdot 10}_{\mathrm{k}-\mathrm{mal}}=10^{k}
$$

Möglichkeiten. Die Null, die hierbei auch als Palindrom entsteht, soll dabei nicht mitgezählt werden (ist keine natürliche Zahl), also ist die Anzahl $U(N)$ dieser ungeradzahlstelligen Palindrome

$$
U(N)=10^{k}-1
$$

Nun fehlen noch die Palindrome mit gerader Stellenzahl. Diese werden nach Variante 1 mit $m=k$ erzeugt. Man muss hierbei jedoch aufpassen, dass die entstehenden Palindrome kleiner bleiben als $N$. Dies ist auf jeden Fall dann der Fall, wenn die aus den ersten $m$ Ziffern von $N$ gebildete Zahl $\hat{N}$ größer ist als die Zahl $Z$, die aus den bei Variante 1 gewählten $m$ Ziffern gebildet wird. Für die Wahl dieser Ziffern (bzw. der Zahl Z) hat man also hier

$$
G(N)=\hat{N}-1
$$

Möglichkeiten. Bilden die gewählten $m$ Ziffern genau die Zahl $\hat{N}$, so muss man schauen, ob das entstehende Palindrom wirklich kleiner als $N$ ist. Ist zum Beispiel $N=1947$, so hat man $\hat{N}=19$ und das zu dieser Ziffernwahl gehörige Palindrom 
1991. Dieses dürfte nicht mitgezählt werden, da es größer als $N$ ist. Für $N=1999$ hingegen ist ebenfalls $\hat{N}=19$, und weil $1991 \leq 1999$, wird dieses Palindrom noch mitgezählt. Je nachdem, ob dieses bei dem gegebenen $N$ klappt oder nicht, muss man also noch ein Palindrom mehr zählen oder nicht. Man setzt hierfür $V(N)=1$, wenn es klappt, oder $V(N)=0$ sonst („V“ steht für „vielleicht noch eines“). Zusammen ergibt dies dann

$$
P(N)=U(N)+G(N)+V(N)=10^{k}-1+\hat{N}-1+V(N) .
$$

Genauso geht man vor, wenn $N$ eine ungerade Stellenzahl $n=2 k+1$ hat. Dann gibt es von den geradzahlstelligen Palindromen kleiner als $N$ gerade $G(N)=10^{k}-1$ Stück (gebildet nach Variante 1 mit $m=k$ ). Bei den Palindromen ungerader Stellenzahl gebildet nach Variante 2 (mit $m=k+1$ ) muss man wieder aufpassen, dass die aus den ersten $(k+1)$ Stellen von $N$ gebildete Zahl $\hat{N}$ größer ist als die aus den gewählten Ziffern gebildete Zahl $Z$, also $U(N)=\hat{N}-1$. Ebenso hat man wieder das $V(N)$. Zusammen ergibt sich genau wieder Formel (1.1). Damit ist man eigentlich fertig.

Um nun noch die beiden Fälle ( $n$ gerade oder ungerade) unter einen Hut zu bringen, kann man die sogenannte Gaußklammer-Funktion $\lfloor$.$\rfloor benutzen. Bei positiven Zah-$ len tut sie nichts weiter, als alle Nachkommastellen zu streichen, wie zum Beispiel $\lfloor 2,31\rfloor=2,\lfloor 9,9\rfloor=9$ oder $\lfloor 6\rfloor=6$.

Unter anderem gilt dann für $n=2 k$ und $n=2 k+1$ :

$$
\left\lfloor\frac{n}{2}\right\rfloor=k .
$$

Um $\hat{N}$ zu erhalten, muss man in beiden Fällen die letzten $k$ Stellen der Zahl $N$ streichen. Dies gelingt durch

$$
\hat{N}=\left\lfloor\frac{N}{10^{k}}\right\rfloor .
$$

(Zum Beispiel ist bei $N=1943$ die Zahl $k=2$, also $\frac{N}{10^{2}}=19,43$. Das heißt aber $\left\lfloor\frac{N}{10^{k}}\right\rfloor=\lfloor 19,43\rfloor=19=\hat{N}$.) Setzt man diese beiden Ergebnisse noch in Gleichung (1.1) ein, so bekommt man die für alle $N$ gültige Formel $^{1}$

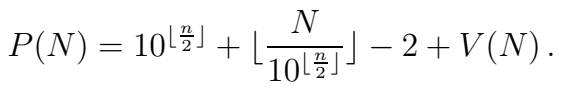

Hiermit kann man nun rechnen, zum Beispiel $P(150)=10+15-2+0=23$ wie in der Aufgabe angegeben. Oder aber auch $P(1234567890)=10^{5}+12345-2+1=112344$.

\footnotetext{
${ }^{1}$ Diese kann man natürlich noch „verbessern“, indem man zum Beispiel $n=\left\lfloor\log _{10} N\right\rfloor+1$ einsetzt. Sogar für $V(N)$ kann man eine nur von $N$ abhängige Formel finden, aber der Aufwand hiefür ist groß und bringt nicht viel.
} 
Um die geforderte Ungleichung zu beweisen kann man nun $\lfloor x\rfloor>x-1$ benutzen. Damit gilt nämlich

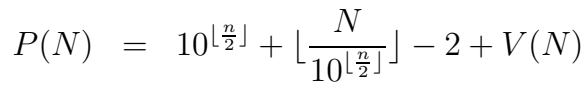

$$
\begin{aligned}
& >10^{\left\lfloor\frac{n}{2}\right\rfloor}+\frac{N}{10^{\left\lfloor\frac{n}{2}\right\rfloor}}-3+V(N) \\
& \geq 10^{\left\lfloor\frac{n}{2}\right\rfloor}+\frac{N}{10^{\left\lfloor\frac{n}{2}\right\rfloor}}-3 \\
& =\left(\sqrt{10^{\left\lfloor\frac{n}{2}\right\rfloor}}-\sqrt{\frac{N}{10^{\left\lfloor\frac{n}{2}\right\rfloor}}}\right)^{2}+2 \sqrt{N}-3 \\
& \geq 2 \sqrt{N}-3
\end{aligned}
$$

Das war zu zeigen. Ein genauerer Vergleich der beiden Funktionen zeigt, dass sie sich an unendlich vielen Stellen $N$ sehr nahe kommen, nämlich an allen Zahlen $N=9999 \ldots 998$ mit einer geraden Anzahl von Stellen. Dies kann man auch anhand obiger Abschätzung beweisen.

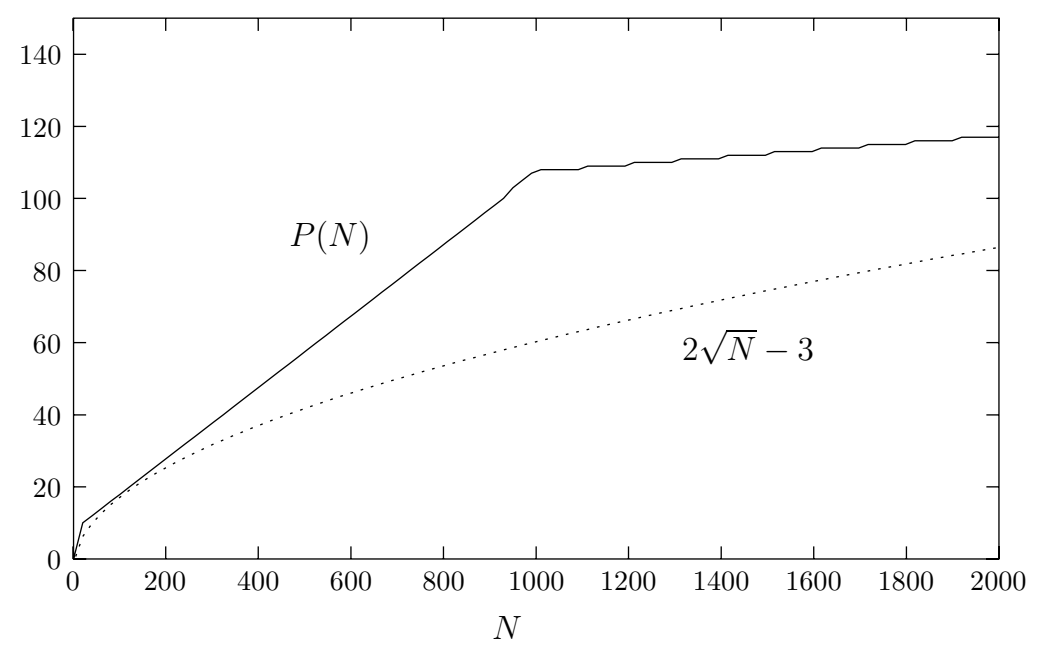

Abbildung 1.3: Vergleich von $P(N)$ mit $2 \sqrt{N}-3$

L 1.4 Vorweg: Einige von euch haben den Vorschlag gemacht, für Kindwurm einen Schlafsack zu nähen. Ein Wurm ist ja recht dünn (sagen wir mal, er habe einen kreisförmigen Querschnitt mit Radius $r$ ), und ein zylinderförmiger Schlafsack hat ja nur eine Oberfläche von $2 \pi r$ (Einheiten wie $\mathrm{m}^{2}$ werden hier der Einfachheit 
halber meist weggelassen). Selbst für $r=0,1$ (dann wäre Würmchen schon ziemlich dick) wären das nur $0,2 \cdot \pi$, also nur ein Fünftel unseres Vorschlages. Wenn man aber so anfängt, muss man auch bedenken, dass der Schlauch an einem Ende geschlossen werden muss, was noch etwas mehr Stoff sowie mehr Nähgeschick erfordert, außerdem ist das Hineinkriechen recht unangenehm, Reißverschlüsse sind jedoch fast unerschwinglich ... Und schließlich ist Deckenstoff im Wurmland deswegen so teuer, weil es ein besonders gut warm haltender Stoff ist (für Wurmkinder sogar gesetzlich vorgeschrieben), der allerdings den Nachteil hat, dass er sich sehr schlecht biegen lässt. (Man hätte ja sonst auch einen recht dünnen Streifen Stoff von einem Meter Länge nehmen und ihn so zurechtbiegen können, dass er die Form der Wurms nachvollzieht. Da wir den Wurm als vernachlässigbar dünn annehmen wollen, bräuchte man also im Prinzip gar keinen Stoff!)

So war das natürlich nicht gemeint, und fast alle haben das ja auch erkannt. Die meisten von euch haben auch die naheliegendste recht gute Lösung angegeben: Eine Kreisscheibe mit Radius $1 / 2$ reicht ebenfalls aus, um Kindwurm zu bedecken (siehe Abbildung 1.4).

Das sieht man am besten folgendermaßen ein: Wenn man die Decke so auf den Wurm legt, dass ihr Mittelpunkt auf der Mitte des Wurms zu liegen kommt, so ist kein Punkt des Wurms mehr als einen halben Meter von diesem Mittelpunkt entfernt, daher liegt er vollständig innerhalb der Kreisscheibe (bzw. ggf. auf deren Rand, der aber zur Kreisscheibe gehören soll). Leider haben viele von euch nicht begründet, warum man Kindwurm mit so einer Decke in jedem Fall zudecken kann; das hätte zur Lösung natürlich eigentlich dazugehört. Das Ergebnis ist auf jeden Fall, dass man für diese Decke nur $(1 / 2)^{2} \cdot \pi \mathrm{m}^{2} \approx 0,785 \mathrm{~m}^{2}$ Stoff benötigt, also nur ein Viertel im Vergleich zu der von uns angegebenen Decke.

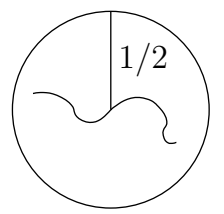

Abbildung 1.4: Decke mit $r=1 / 2$

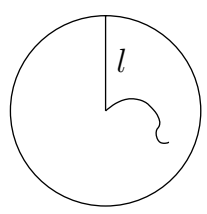

Abbildung 1.5: Ein Wurmende fest

Will man noch bessere Ergebnisse erzielen, muss man mehr Überlegungen anstellen. Auf den Bildern 1.8 und 1.9 seht ihr mögliche andere Deckenformen. Man kann sich den Wurm (nur gedanklich!) in einzelne Abschnitte unterteilen und sich überlegen, welche Form man braucht, um einen dieser Abschnitte bei einer vorgegebenen Lage der beiden oder eines Endpunkte(s) zu überdecken. Der Fall, dass man die Lage nur eines Endpunktes als fix betrachtet, ist oben bzw. auf dem Aufgabenblatt schon gelöst worden: Geht von einem Punkt $A$ ein Wurmstück der Länge $l$ aus, so liegt dieses garantiert vollständig in einer Kreisscheibe um $A$ mit dem Radius $l$. 
Etwas kniffliger wird es, wenn man die Lage beider Endpunkte $A$ und $B$, die einen Abstand $d$ voneinander haben mögen, gegeben hat: Dann liegt das Wurmstück (wieder mit der Länge $l$ ) garantiert in einer Ellipsenscheibe mit den Brennpunkten $A$ und $B$ und mit großer Halbachse $l / 2$. Daraus ergibt sich nach Pythagoras eine kleine Halbachse der Länge $\sqrt{(l / 2)^{2}-(d / 2)^{2}}$. Mit diesem Wert lässt sich nicht mehr so schön rechnen, aber für unsere Abschätzungen reicht es zu wissen, dass die kleine Halbachse nicht länger als $l / 2$ ist. Man denke sich nun die Punkte des Wurmes bei einem Viertel $(A)$ sowie bei drei Vierteln $(B)$ seiner Länge markiert und lege den Wurm so auf eine gegebene Achse, dass $A$ auf dem (selbst gewählten) Nullpunkt der Achse liegt, $B$ rechts davon ebenfalls auf der Achse.

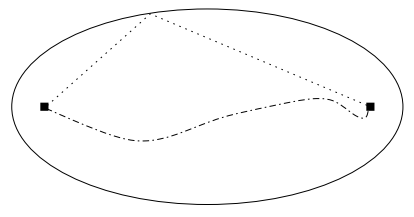

Abbildung 1.6: Beide Wurmenden fest

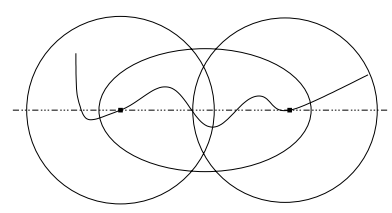

Abbildung 1.7: Wurm bei 1/4 und 3/4 fest

Dann liegt der Wurm garantiert in der Figur, die durch die Überlagerung zweier Kreisscheiben mit Radius 1/4 um $A$ und $B$ sowie der Ellipsenscheibe mit den Brennpunkten $A$ und $B$ und der großen Halbachse 1/4 entsteht. Man beachte, dass die Ellipse rechts und links nicht aus den Kreisscheiben „herausstoßen“ kann, da ihr Mittelpunkt zwischen $A$ und $B$ liegt und die große Halbachse nur genauso groß wie die Kreisradien ist. Ebenso liegt kein Punkt der Ellipse weiter als $1 / 4$ von der Achse entfernt, weil die kleine Halbachse kleiner oder gleich $1 / 4$ ist. In den beiden Extremfällen sind $A$ und $B$ gleich bzw. liegen im Abstand von 1/2 zueinander.

Das bedeutet aber, dass man den Wurm, egal wie er sich gekrümmt hat, vollständig von der Figur in Abbildung 1.8 bedecken kann, da in ihr alle möglichen oben konstruierten Kreis-Ellipse-Kreis-Gebilde (also für jeden möglichen Abstand zwischen $A$ und $B$ ) enthalten sind: Ein Quadrat mit der Seitenlänge 1/2, daran anliegend an zwei gegenüberliegenden Seiten je ein Halbkreis mit Radius 1/4. Der Flächeninhalt dieser Figur beträgt $(0,5)^{2}+2 \cdot 0,5 \cdot \pi(0,25)^{2} \approx 0,446$, was

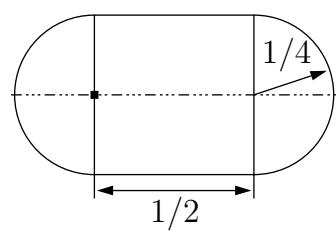

Abbildung 1.8: Decke mit $A \approx 0,446$ noch deutlich besser ist als der kleinere Kreis. Bis zu welcher Länge der kleinen Halbachse (bei großer Halbachse 1/2) eine reine Ellipsenform eine mögliche Lösung ist, ist uns nicht bekannt. Zumindest geht es noch für 0,319, weil dann noch der unten vorgestellte Halbkreis in der Figur enthalten ist. Ein Vorschlag eines Teilnehmers war, für die kleine Halbachse die Länge $1 /(2 \pi)(\approx 0,159)$ zu nehmen. (In diese Ellipse würde der Wurm immerhin genau hineinpassen, wenn er sich zu einem Kreis 
rollt.) Wer Lust hat, kann versuchen, sich zu überlegen, dass dies zu klein ist. (Tipp: Der Wurm kann nicht überdeckt werden, wenn er sich zu einem rechten Winkel mit zwei Schenkeln der Länge $1 / 2$ legt.)

Die beste uns bekannte Lösung ist genau halb so groß wie der Kreis mit Radius 1/2, es ist nämlich genau ein Halbkreis davon. Der Beweis, dass dies reicht, benutzt wie oben die Prinzipien der Kreis- bzw. der Ellipsenscheibe (siehe Abbildung 1.9).
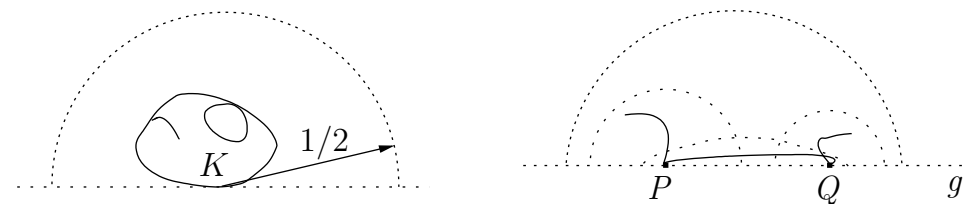

Abbildung 1.9: Zum Beweis für die kleinste uns bekannte Decke $(A \approx 0,393)$

Eine Gerade, die den Wurm berührt, aber nicht schneidet, heiße Stützgerade. Wenn es nur Stützgeraden gibt, die den Wurm in genau einem Punkt berühren, so liegt dieser in der Form einer konvexen Schleife mit eventuell zusätzlich nach innen hin liegenden Abschnitten, also mit einem Umfang kleiner gleich 1. Man wähle sich eine der Stützgeraden aus, und der Berührpunkt heiße $K$. Jeder Punkt des Wurms hat einen Abstand von kleiner oder gleich $1 / 2$ von $K$. (Man kann sich einem Punkt auf dem Rand ja nach Belieben von beiden Seiten nähern und den kürzeren Weg wählen; für innere Punkte ist die Aussage dann klar.) Damit liegt Kindwurm in einem Kreis um $K$ mit Radius 1/2. Da er aber vollständig auf einer Seite der Geraden liegt, liegt er auch in einem Halbkreis mit Radius $1 / 2$.

Wenn der eben behandelte Fall nicht eintritt, gibt es eine Stützgerade $g$, die den Wurm in zwei Punkten $P$ und $Q$ (und eventuell noch weiteren) berührt. Diese teilen ihn der Reihe nach in drei Teilstücke $A, B$ und $C$ mit den Längen $a, b$ und $c$ (mit $a+b+c=1)$ ein. Der Abstand $d$ zwischen $P$ und $Q$ ist offensichtlich $d \leq b$. Den oben durchgeführten Überlegungen entsprechend liegt $A$ in einem Kreis um $P$ mit Radius $a$. Da $A$ aber auf nur einer Seite von $g$ liegt, liegt es auch in einem Halbkreis um $P$ mit Radius $a$. Genauso liegt $C$ in einem Halbkreis (auf der gleichen Seite von $g$ wie der andere) um $Q$ mit Radius $c$. Und genauso entsprechend liegt $B$ in einer halben Ellipse mit den Brennpunkten $P$ und $Q$ und großer Halbachse $b$.

Der ganze Wurm liegt also innerhalb einer Figur, die aus der Überlappung zweier Halbkreise und einer Halbellipse entsteht. Seien $X$ und $Y$ die Extremalpunkte dieser Figur auf $g$. Da die Halbellipse die Breite $b$ hat und die Halbkreise die Radien $a$ und $c$, ist der Abstand zwischen $X$ und $Y$ kleiner gleich $a+b+c=1$. Nun ist die Halbellipse „flacher" als ein Halbkreis der gleichen Breite, und alle Halbkreise haben einen Radius kleiner als 1/2, daher liegt die gesamte Figur in einem Halbkreis mit Durchmesser 1 , was zu beweisen war.

Wer sich noch mehr für dieses Thema interessiert, kann in dem Buch „Spiel, Satz, Sieg für die Mathematik" von Ian Stewart weiterlesen. 


\section{Lösungen zu Aufgabenblatt 2}

L 2.1 Die eigentliche Aufgabe war durch Rückwärtsrechnen lösbar: Der letzte Räuber lässt 78 Münzen übrig. Diese entsprechen drei Vierteln der Anzahl an Münzen, die er glatt teilen konnte (das waren also $\frac{4}{3} \cdot 78=104$ ), und um überhaupt glatt teilen zu können, hatte er eine Münze entfernen müssen, also fand er $104+1=$ 105 Münzen vor. Entsprechend fanden der dritte Räuber $\left(\frac{4}{3} \cdot 105\right)+1=141$, der zweite $\left(\frac{4}{3} \cdot 141\right)+1=189$ und der erste Räuber $\left(\frac{4}{3} \cdot 189\right)+1=253$ Münzen vor. Es waren also ursprünglich 253 Münzen in dem Schatz.

In der Zusatzaufgabe war nun gefordert, eine Anzahl Münzen zu finden, die fünf Räuber unter sich auf die gleiche Weise aufteilen würden, das heißt, dass jeder der fünf erst einmal eine Münze entfernen muss, bevor er den (Rest-)Schatz glatt aufteilen kann. Weil die Anzahl an Münzen damit noch nicht eindeutig bestimmt ist (denn man kann beliebig oft noch eine Anzahl Münzen dazutun, die bei der Räuber-Teilung jedes Mal ohne Rest aufgeteilt werden kann [zum Beispiel $5^{5}$; man kann sich sogar überlegen, dass dafür genau die Vielfachen von $5^{5}$ geeignet sind]), war zusätzlich gefordert, die Anzahl zu finden, die den kleinsten Rest übrig lässt (was natürlich gleichbedeutend damit ist, die kleinstmögliche Anfangsanzahl zu finden). Der naheliegendste Lösungsansatz ist, auch hier rückwärts zu rechnen. (Später kommt noch eine elegantere Variante.) Es bleibe also eine Anzahl $r$ an Münzen übrig. Dann hat der letzte Räuber $\frac{5}{4} r+1=\frac{5 r+4}{4}$ Münzen gefunden, der vierte $\frac{5}{4}\left(\frac{5 r+4}{4}\right)+1=\frac{5^{2} r+5 \cdot 4+4^{2}}{4^{2}}$, der dritte $\frac{5^{3} r+5^{2} \cdot 4+5 \cdot 4^{2}+4^{3}}{4^{3}}$, der zweite $\frac{5^{4} r+5^{3} \cdot 4+5^{2} \cdot 4^{2}+5 \cdot 4^{3}+4^{4}}{4^{4}}$ und der erste $\frac{5^{5} r+5^{4} \cdot 4+5^{3} \cdot 4^{2}+5^{2} \cdot 4^{3}+5 \cdot 4^{4}+4^{5}}{4^{5}}=\frac{5^{5} r+4 \cdot 2101}{4^{5}}$. Jetzt muss man also das $r$ so bestimmen, dass $5^{5} r+4 \cdot 2101$ durch $4^{5}$ teilbar ist.

Weil $5^{5}$ nicht durch 4 teilbar ist, muss $r=4 s$ mit einem ungeraden $s$ sein. Nun könnte man für $s$ der Reihe nach 1, 3, 5, 7 usw. einsetzen und feststellen, dass (erst!) $s=255$ eine Lösung ist. Es geht aber etwas einfacher, wenn man sich die letzte Teilaufgabe umformt:

$4^{5}=1024$ teilt $5^{5} r+4 \cdot 2101=3125 \cdot 4 s+4 \cdot 2101=(12 \cdot 1024+4 \cdot 53) s+8 \cdot 1024+4 \cdot 53$

$\Leftrightarrow 4^{5}$ teilt $4 \cdot 53 s+4 \cdot 53$

$\Leftrightarrow 4^{4}$ teilt $53 s+53$

$\Leftrightarrow 4^{4}$ teilt $s+1$.

Daher ist das kleinste (positive) $s$, das eine Lösung ist: $s=4^{4}-1=255$. Es folgt $r=4 \cdot 255=1020$, das ist also die kleinste mögliche Anzahl an übrig bleibenden Münzen im Schatz. Am Anfang waren dann übrigens $\frac{3125 \cdot 1020+4 \cdot 2101}{1024}=3121$ Münzen vorhanden.

Nun noch die elegante Methode: Es kann einem auffallen, dass bei der ersten Aufgabe gilt: $253=4^{4}-3$ sowie $78=3^{4}-3$. Also könnte man auf die Idee kommen, dass man bei fünf Räubern ähnliche Zahlen, namentlich $5^{5}-4=3121$ und $4^{5}-4=1020$ 
herausbekommt. Daher rechne man jetzt nicht mit $r$, sondern mit $\tilde{r}:=r+4$. (Es sollen also am Schluss $\tilde{r}-4$ Münzen übrig bleiben.) Dann hat der fünfte Räuber $\frac{5}{4}(\tilde{r}-4)+1=\frac{5}{4} \tilde{r}-4$ Münzen vorgefunden. Entsprechend der vierte $\frac{5^{2}}{4^{2}} \tilde{r}-4$, der dritte $\frac{5^{3}}{4^{3}} \tilde{r}-4$, der zweite $\frac{5^{4}}{4^{4}} \tilde{r}-4$ und der erste $\frac{5^{5}}{4^{5}} \tilde{r}-4$. Hier sieht man sofort, dass die kleinste Lösung $\tilde{r}=4^{5}=1024$ ist. Die kleinste Restmünzenanzahl ist also wie vermutet $1024-4=1020$.

L 2.2 Da die Treppe den Fernsehturm auf seiner vollen Höhe von $100 \mathrm{~m}$ genau zwanzig Mal umrundet, umrundet sie ihn auf $\frac{100 \mathrm{~m}}{20}=5 \mathrm{~m}$ genau einmal. Da der Durchmesser des Turmes $10 \mathrm{~m}$ beträgt und der Läufer in einem Meter Abstand die Treppe hochlaufen soll, liegt sein Weg auf einem Zylinder mit dem Durchmesser $(1+10+1) \mathrm{m}$. Nun stelle man sich vor, daß man ein fünf Meter hohes Teilstück dieses Zylinders aufschneiden und abrollen kann. Man erhält so ein Rechteck mit den Seitenlängen $a=12 \pi \mathrm{m}$ und $b=5 \mathrm{~m}$. Zeichnet man noch den Weg des Läufers auf der Treppe ein, so erhält man ihn als Diagonale in dem Rechteck.
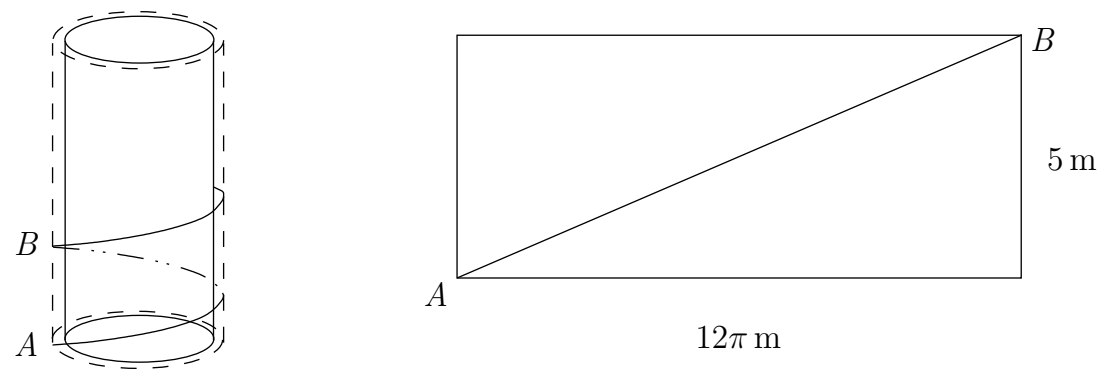

Nach dem Satz des Pythagoras $\left(c^{2}=a^{2}+b^{2}\right)$ berechnet man die Strecke $c$ des Läufers auf diesem $5 \mathrm{~m}$ hohen Teilstück. Die Gesamtstrecke $s$ des Läufers beträgt dann $s=20 c$.

$$
c^{2}=(5 \mathrm{~m})^{2}+(12 \pi \mathrm{m})^{2}
$$

bzw.

$$
s=20 c=20 \sqrt{25+(12 \pi)^{2}} \mathrm{~m} \approx 760,58 \mathrm{~m} .
$$

Der Läufer legt auf seinem Weg nach oben also 760,58 m zurück.

Die zurückgelegte Wegstrecke ist also abhängig von der Höhe des Turms. Einige Einsender hatten das fälschlicherweise nicht berücksichtigt und so getan, als wäre die Strecke genauso lang, wenn der Läufer 20-mal unten um den Turm läuft. Die Länge dieser Strecke beträgt ca. 753 Meter, und das Ergebnis würde sich nicht ändern, wenn die Treppe 20-mal um einen Turm der (gedachten) Höhe von 1000 Meter führt. Das hieße aber, dass man auf der Treppe schneller nach oben käme als auf direktem Wege senkrecht an der Wand hoch, was nicht sein kann. 


\section{2.3}

a) Wenn man anfängt zu probieren, stellt man bald Folgendes fest: Für die meisten Zahlen ist die Summe der Quadrate der Ziffern kleiner als die Zahl selbst. Zum Beispiel ist $1^{2}+3^{2}=10<13,3^{2}+9^{2}+6^{2}=126<396$. Nur für "wenige“ Zahlen ist es anders, ein Beispiel ist $3^{2}+8^{2}=73>38$. Die Idee besteht nun darin zu beweisen, dass ab einer bestimmten natürlichen Zahl $n$ alle Zahlen, die größer sind, die erste Eigenschaft haben. Dann wäre klar, dass keine Zahl größer als $n$ gleich der Summe der Quadrate ihrer Ziffern ist. Die Zahlen kleiner als $n$ probiert man einzeln durch, z. B. mit einem Computer. Auch ohne Computer ist dies mit vertretbarem Zeitaufwand möglich, wenn man dabei noch Hilfsüberlegungen anstellt, mit denen man jeweils einige Fälle gleichzeitig bearbeiten kann.

Für vierstellige natürliche Zahlen $x$ gilt: $x \geq 1000$. Außerdem ist jede Ziffer von $x$ eine der Zahlen $0,1,2, \ldots, 9$. Die Summe $s$ der Quadrate der Ziffern ist also höchstens $4 \cdot 9^{2}=324$ und damit immer kleiner als 1000 .

Allgemein gilt so für eine Zahl $x$ mit $m$ Stellen: $10^{m-1} \leq x$, für die Summe $s$ der Quadrate der Ziffern gilt $s \leq m \cdot 9^{2}$. Und für $m \geq 4$ gilt $^{1}$ :

$$
m \cdot 9^{2}<10^{m-1}
$$

und damit $s<x$. Also kann bei keiner vier- oder mehrstelligen Zahl die Summe der Quadrate der Ziffern gleich der Zahl selbst sein. Die Zahlen kleiner 1000 probiert man einzeln durch. Wenn man noch bedenkt, dass $3 \cdot 9^{2}=243$ ist, braucht man also nur die Zahlen bis $243 \mathrm{zu}$ testen. (Warum?) Dabei stellt man fest, dass nur bei der Zahl 1 die Forderung erfüllt ist.

b) Hier geht man völlig analog vor. Für $m \geq 5$ gilt:

$$
m \cdot 9^{3}<10^{m-1}
$$

Fünf- und mehrstellige Zahlen können somit nicht gleich der Summe der Kuben ihrer Ziffern sein. Beachtet man noch $4 \cdot 9^{3}=2916$, so reicht es, alle Zahlen kleiner 2916 durchzutesten. Man stellt fest, dass die Bedingung genau für die fünf Zahlen

$$
1,153,370,371 \text { und } 407
$$

erfüllt ist, z. B. ist $3^{3}+7^{3}+0^{3}=27+343+0=370$.

L 2.4 Vornweg: Die Aufgabe (bzw. das Ergebnis) ist als Buffons Nadelproblem bekannt und wird gelegentlich im Rahmen von Kursen zur Wahrscheinlichkeitsrechnung in der Oberstufe am Gymnasium oder auch an der Uni gestellt. Das Ergebnis

\footnotetext{
${ }^{1}$ Für Interessierte der Beweis durch Induktion: Induktionsanfang für $m=4: 4 \cdot 9^{2}=324<$ $1000=10^{4-1} ;$ Induktionsschritt: $(m+1) \cdot 9^{2}<10^{m-1}+9^{2}$ (Ind.-Voraussetzung) $<$ $10^{m-1+1}\left(\right.$ da $9^{2}=81<10^{m-1}$ für $m \geq 3$ )
} 
Lösungen zu Aufgabenblatt 2

ist recht verblüffend und wird meist mit Hilfe der Integralrechnung bewiesen (wie es auch einige der älteren Einsender taten). Es gibt jedoch auch einen Beweis, der ohne solche „komplizierten“ Hilfsmittel auskommt und auch für jüngere Schüler verständlich sein sollte. Im Folgenden werden beide vorgestellt:

Ergebnis: Die Wahrscheinlichkeit, dass die Nadel eine Linie trifft, ist $p=\frac{2}{\pi} \approx$ 0,63662. Damit ist dann $\alpha=\frac{2}{p}=\pi \approx 3,14159265$.

Beweis 1: Die möglichen Positionen der Nadel kann man durch zwei Parameter beschreiben: den Abstand $x$ des Mittelpunktes $M$ der Nadel zu den Gitterlinien (und zwar den minimalen) und den Winkel $\phi$, um den die Nadel dazu aus der Parallellage im Gitter gedreht ist.

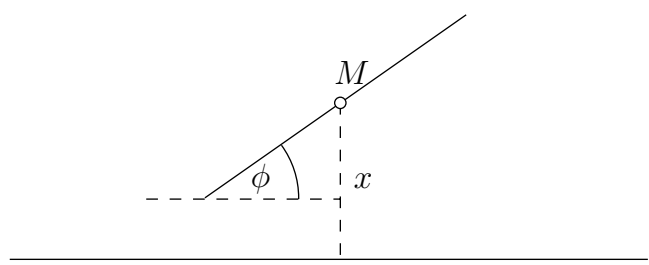

Hierbei kann $x$ alle Werte zwischen 0 und $\frac{l}{2}$ und $\phi$ alle Werte zwischen 0 und $\pi$ (also zwischen $0^{\circ}$ und $180^{\circ}$ ) annehmen. Welches sind nun die Parameter, bei denen die Nadel eine Linie trifft? Obigem Bild folgend trifft die Nadel genau dann eine Linie, wenn

$$
x \leq \sin \phi \cdot \frac{l}{2}
$$

ist. Man kann sich diese Bedingung auch in einem Koordinatensystem veranschaulichen.

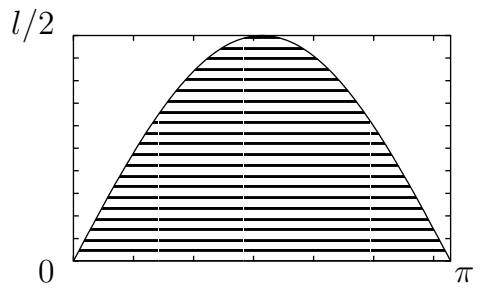

Die schraffierte Fläche unter der Sinuskurve kennzeichnet dabei genau die Punkte, die (2.1) erfüllen. Geht man nun davon aus, dass wirklich jeder dieser Punkte gleich wahrscheinlich ist (dass also jeder mögliche Winkel und jeder mögliche Abstand unabhängig voneinander gleich wahrscheinlich sind), so ist die gesuchte Trefferwahrscheinlichkeit gerade der Anteil der schraffierten Fläche an der Gesamtfläche. Hier 
kommt nun die Integration ins Spiel ...

$$
p=\frac{\text { Fläche }_{\text {schraffiert }}}{\text { Fläche }_{\text {gesamt }}}=\frac{\int_{0}^{\pi} \frac{l}{2} \sin \phi d \phi}{\frac{l}{2} \cdot \pi}=\frac{-\left.\cos \phi\right|_{0} ^{\pi}}{\pi}=\frac{2}{\pi}
$$

Und nun der Beweis, der ohne Integration auskommt:

Beweis 2: Hierbei werfe man zunächst eine Nadel beliebiger Länge $L$ auf das Gitter der Breite $l$. Dabei kann die Nadel das Gitter nun durchaus auch mehrfach treffen. Sei $X$ die Anzahl der Schnittpunkte bei einem Wurf. Mit einer gewissen Wahrscheinlichkeit $p_{0}$ trifft die Nadel keine Linie (in Formeln: $P(X=0)=p_{0}$ ), mit einer anderen Wahrscheinlichkeit $p_{1}$ trifft die Nadel genau eine Linie (in Formeln: $\left.P(X=1)=p_{1}\right)$ usw. Wie viele Schnittpunkte erwartet man nun durchschnittlich pro Wurf? Dies gibt gerade der sogenannte Erwartungswert

$$
E(X)=0 \cdot p_{0}+1 \cdot p_{1}+2 \cdot p_{2}+\ldots=p_{1}+2 p_{2}+3 p_{3}+\ldots\left(=\sum_{k=0}^{\infty} P(X=k) \cdot k\right)
$$

wieder. $^{2}$

Für unsere Nadel, die ja genau so lang ist, wie das Gitter breit ist, gilt $0=p_{2}=$ $p_{3}=\ldots$, da ja nie mehr als ein Schnittpunkt auftreten kann. Hier ist also der Erwartungswert $E(X)=p_{1}$ gerade die gesuchte Wahrscheinlichkeit. Um diese berechnen zu können, müssen wir einen kleinen „Umweg“ machen. Dabei ist es nötig, beliebig gebogene Drähte (die man sich als aus vielen kleinen Nadeln zusammengesetzt vorstellen kann) auf das Gitter zu werfen. Die Schritte auf dem Weg dahin sind in folgenden Punkten zusammengefasst:

- Der Erwartungswert $E(X)$ ist abhängig von der Länge $L$ der Nadel, also eine Funktion von $L$. Man kann dies als $E(X)=E(L)$ schreiben. Ebenso sollte klar sein, dass $E(L)$ eine monoton steigende Funktion ist, also je länger die Nadel, umso höher der Erwartungswert für die Trefferzahl.

- Man betrachte nun zwei Nadeln der Längen $L_{1}$ und $L_{2}$, die man getrennt auf das Gitter wirft, und $X_{1}$ und $X_{2}$ seien wie bisher die Anzahlen der Schnittpunkte eines Wurfes. Will man nun die Summe der Treffer bei beiden Nadeln betrachten, so ist es sicher nicht schwer, die Formel

$$
E\left(X_{1}+X_{2}\right)=E\left(X_{1}\right)+E\left(X_{2}\right)
$$

zu glauben. Die Zahl, die man im Mittel als Trefferanzahl pro Wurf mit beiden Nadeln erwartet, ist sicher gerade die Summe der einzelnen Erwartungswerte.

\footnotetext{
${ }^{2}$ Man überlege sich dies am Beispiel des Würfelwurfs $X$. Hierbei gibt es die sechs möglichen Ergebnisse $X=1$ bis $X=6$ und für jedes mögliche $k$ ist $P(X=k)=\frac{1}{6}$. Der Erwartungswert hierfür ist also gerade $E(X)=\frac{1}{6} \cdot 1+\ldots+\frac{1}{6} \cdot 6=3,5$. Im Mittel erwartet man also das Ergebnis 3,5 .
} 
Etwas mehr Überlegung muss man investieren, um Folgendes einzusehen: Die letzte Formel gilt auch noch, wenn man die beiden Nadeln nicht getrennt wirft, sondern an ihren Enden starr verbindet, ganz egal wie - ob im Winkel oder gestreckt.

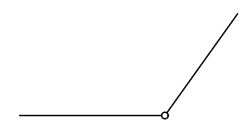

Eine mögliche Argumentation hierfür wäre: Beim Werfen und anschließenden Auswerten beachte man die jeweils andere Nadel einfach nicht, man betrachte sie als "Anhängsel". Damit sollte es zunächst egal sein, ob man die Nadeln einzeln nacheinander oder zusammengeklebt zweimal wirft und dabei jeweils nur eine der Nadeln beachtet. Im letzten Schritt muss man sich klarmachen, dass der Erwartungswert sich dann auch nicht mehr ändert, wenn man die Trefferzahlen der beiden Nadeln sofort bei nur einem Wurf auswertet. (Diesen Sachverhalt - Linearität des Erwartungswertes - kann man ganz allgemein beweisen. Hier sollte diese Argumentation aber genügen.)

- Aus der letzten Überlegung folgt nun für die Abhängigkeit von den Längen:

$$
E\left(L_{1}+L_{2}\right)=E\left(L_{1}\right)+E\left(L_{2}\right) .
$$

Wenn dies für zwei Nadelstücke gilt, so sicher auch für jede beliebige Anzahl $n$ :

$$
E\left(L_{1}+L_{2}+L_{3}+\ldots+L_{n}\right)=E\left(L_{1}\right)+E\left(L_{2}\right)+\ldots+E\left(L_{n}\right)
$$

Noch einmal: Letztere Formel gilt für $n$ Nadeln der entsprechenden Längen, die irgendwie zu einem Streckenzug verbunden wurden. Die einzigen Funktionen $E$, die sowohl monoton sind als auch die Gleichung (2.2) erfüllen, sind die so genannten linearen Funktionen ${ }^{3}$

$$
E(L)=c \cdot L
$$

mit einer Konstanten $c$. Der Trick besteht nun darin, dass man mit beliebig vielen kurzen Nadeln einen Kreis mit Durchmesser $l$ beliebig genau annähern kann. Für diesen muss also auch die Formel (2.3) gelten. Hier kennt man aber sowohl $L=\pi \cdot l$ als auch $E=2$, denn egal wie man einen Kreis mit Durchmesser $l$ auch auf das Gitter wirft, er trifft die Linien stets genau zweimal.

\footnotetext{
${ }^{3}$ Beweis: Setzt man $f(1)=: c$, so gilt $f(2)=f(1)+f(1)=2 c, f(3)=3 c$ und allgemein $f(k)=k \cdot c$. Für Brüche folgt dann: $n \cdot f\left(\frac{m}{n}\right)=f(m)=m \cdot c$, also $f\left(\frac{m}{n}\right)=\frac{m}{n} \cdot c$. Damit gilt $f(x)=c \cdot x$ für alle positiven rationalen Zahlen. Für die negativen folgt es wegen $f(x)+f(-x)=f(0)=0$ (weil $f(0)+f(0)=f(0)$ ). Aus der Monotonie folgt dann, dass $f$ keine „Sprünge" machen darf, also $f(x)=c \cdot x$ für alle reellen Zahlen.
} 


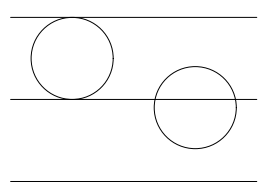

Der Erwartungswert ist also genau 2. Setzt man dies in Formel (2.3) ein, so folgt:

$$
2=c \cdot l \cdot \pi
$$

bzw.

$$
c=\frac{2}{l \pi}
$$

Die allgemeine Formel ist also

$$
E(L)=\frac{2}{l \pi} \cdot L
$$

Für unser Ausgangsproblem mit nur einer Nadel der Länge $l$ erhält man damit

$$
p=E(l)=\frac{2}{\pi}
$$

Nachwort: Einige von euch haben erkannt, dass der berechnete Wert $\alpha$ ungefähr $\pi$ war. Bei vielen gab es aber recht große Abweichungen. Dies ist nicht weiter verwunderlich, da es sich ja immer nur um Wahrscheinlichkeiten handelt. Man muss schon sehr oft werfen, damit sich die Häufigkeit der Wahrscheinlichkeit gut annähert. Nimmt man alle eure Würfe zusammen - 4179 Stück -, so gab es 2724 Treffer. Damit erhält man den Wert

$$
\alpha \approx 3,068 \text {. }
$$

Auch dieser erinnert noch nicht unbedingt an $\pi$. Es gibt aber zum Beispiel Computerprogramme, die das Werfen simulieren. Mit diesen erhält man ganz gute Werte für $\pi$. 


\section{Lösungen zu Aufgabenblatt 3}

L 3.1 Die Lösung stammt u. a. von Manuel und Robert:

Von 1000 Menschen haben 35 zu hohen Blutdruck, 965 nicht.

80 Prozent derjenigen mit hohem Blutdruck trinken, das sind 28 Personen.

60 Prozent derjenigen ohne hohen Blutdruck trinken ebenfalls, das sind 579 Personen.

Zusammen trinken also 607 Personen regelmäßig.

Da von diesen $28 \mathrm{zu}$ hohen Blutdruck haben, entspricht das

$$
\frac{28}{607} \cdot 100 \approx 4,61 \% \text {. }
$$

Die gesuchte Zahl ist also 4,61 Prozent.

L 3.2 Behauptung: Die gesuchte Zahl ist 2519.

Beweis: Das kleinste gemeinsame Vielfache von $n$ ganzen Zahlen $x_{1}, x_{2}, \ldots, x_{n}$ ist die kleinste positive ganze Zahl, die durch jede der Zahlen $x_{1}, x_{2}, \ldots, x_{n}$ ohne Rest teilbar ist. Wir schreiben für diese Zahl $\mathrm{kgV}\left(x_{1}, \ldots, x_{n}\right)$.

$\mathrm{Da} \operatorname{kgV}(2,3,4,5,6,7,8,9,10)=9 \cdot 8 \cdot 7 \cdot 5=2520$ gerade ohne Rest durch $2,3,4,5$, $\ldots, 9,10$ teilbar ist, lässt 2519 bei Division durch $k$, wie gefordert, den Rest $k-1$ für jede der Zahlen $k=2,3, \ldots, 9,10$.

Und dies ist auch die kleinste derartige Zahl, denn: Sei $n$ eine positive ganze Zahl, die bei Division durch 2 den Rest 1, durch 3 den Rest 2, durch 4 den Rest 3, durch 5 den Rest 4, durch 6 den Rest 5, durch 7 den Rest 6, durch 8 den Rest 7, durch 9 den Rest 8 und durch 10 den Rest 9 lässt.

Dann ist $n+1$ durch 2,3,4,5, ., 9, 10 jeweils ohne Rest teilbar (d.h. mit Rest 0 ), muss also größer/gleich dem kleinsten gemeinsamen Vielfachen der Zahlen sein. Also ist 2519 tatsächlich die kleinste derartige Zahl.

L 3.3 Behauptung: Der geometrische Ort der Höhenschnittpunkte ist der Kreis $k^{\prime}$, der aus $k$ durch Spiegelung an der Geraden $(A B)$ entsteht.

Beweis: Im linken Bild sei $C$ ein beliebiger Punkt auf $k$ so, dass das Dreieck $A B C$ nicht spitzwinklig ist, und $H$ der Höhenschnittpunkt dieses Dreiecks.

Mit den Winkelbezeichnungen in der Skizze gilt dann:

$$
\begin{aligned}
& \text { Im Dreieck } A B D: \quad \alpha+\angle C B A+90^{\circ}=180^{\circ} \Rightarrow \alpha=90^{\circ}-\angle C B A \\
& \text { Im Dreieck } A B E: \quad \beta+\angle B A C+90^{\circ}=180^{\circ} \Rightarrow \beta=90^{\circ}-\angle B A C
\end{aligned}
$$

Daraus folgt, dass im Dreieck $A B H$ gilt:

$$
\delta+\alpha+\beta=180^{\circ}
$$



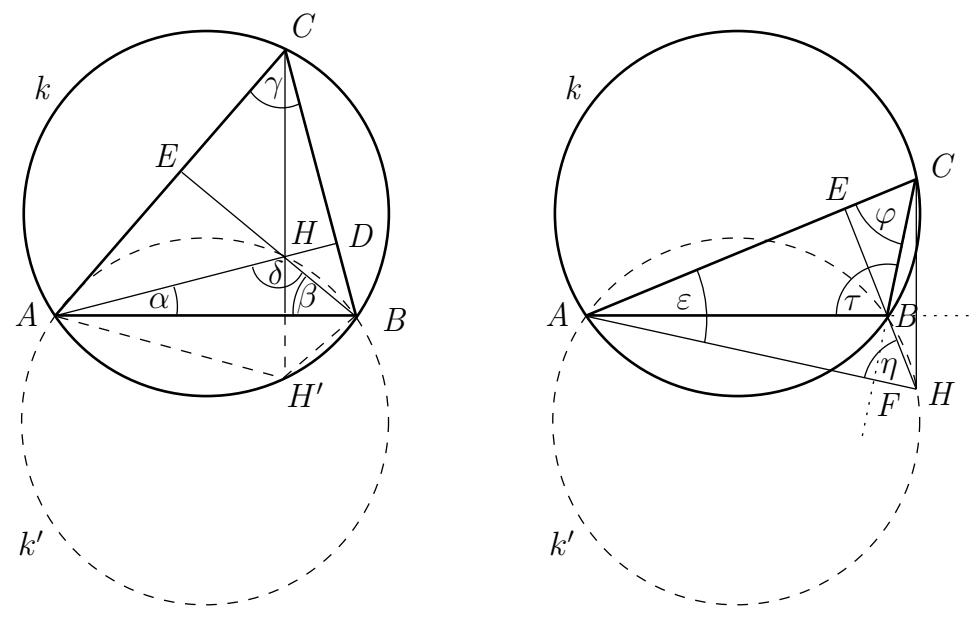

$$
\Rightarrow \delta=180^{\circ}-\left(90^{\circ}-\angle C B A\right)-\left(90^{\circ}-\angle B A C\right)=\angle C B A+\angle B A C
$$

Wegen $\angle C B A+\angle B A C=180^{\circ}-\gamma$ folgt also schließlich:

$$
\delta=180^{\circ}-\gamma
$$

Nach der Umkehrung des Satzes vom Sehnenviereck ${ }^{1}$ gilt dann, dass $H^{\prime}$, der an $(A B)$ gespiegelte Punkt $H$, auf dem Kreis $k$ liegen muss (siehe Bild). Anders ausgedrückt heißt das, dass $H$ auf dem Kreis $k^{\prime}$ liegt, der aus $k$ durch Spiegelung an $(A B)$ hervorgeht.

Sei nun das Dreieck $A B C$ stumpfwinklig mit stumpfem Winkel bei $B$ (für den Fall, dass der stumpfe Winkel bei $A$ liegt, funktioniert die Argumentation genau entsprechend) - siehe dazu die rechte Skizze. Hier gilt:

$$
\begin{array}{ll}
\text { Im Dreieck } A F C: & \varphi+\varepsilon+90^{\circ}=180^{\circ} \Rightarrow \varphi=90^{\circ}-\varepsilon \\
\operatorname{Im} \text { Dreieck } A H E: & \varepsilon+\eta+90^{\circ}=180^{\circ} \Rightarrow \eta=90^{\circ}-\varepsilon=\varphi .
\end{array}
$$

Nach der Umkehrung des Peripheriewinkelsatzes liegt folglich $H$ auf einem Kreis, der durch $A$ und $B$ verläuft und den gleichen Radius wie der Umkreis des Dreiecks $A B C$ hat. Aus den Lagebeziehungen folgt, dass dies nicht $k$ selbst sein kann, also liegt $H$ wiederum auf $k^{\prime}$.

Für den dritten und letzten Fall, dass nämlich das Dreieck bei $C$ einen stumpfen Winkel hat, kann man wieder die linke Skizze verwenden: Man denke sich $C$ am Ort von $H^{\prime}$ liegend und dazu das Dreieck $A B C$ mit seinen Höhen an $(A B)$ gespiegelt.

\footnotetext{
${ }^{1}$ Ist in einem Viereck die Summe gegenüberliegender Winkel $180^{\circ}$, so handelt es sich um ein Sehnenviereck (also ein Viereck, dessen vier Ecken alle auf einem Kreis liegen).
} 
Lösungen zu Aufgabenblatt 3

Das Bild von $C$ ist dann offenbar der Höhenschnittpunkt, und dieser liegt erneut auf $k^{\prime}$.

Man erkennt somit insgesamt, dass der Höhenschnittpunkt in jedem Fall auf die gleiche Art bestimmt werden kann, nämlich durch die Verschiebung von $C$ um eine feste Strecke senkrecht zu $(A B)$. Da $C$ alle Punkte auf $k$ einnehmen kann, wird von den Höhenschnittpunkten auch jeder Punkt von $k^{\prime}$ erreicht. (Wenn man es genau nimmt, müssten wir zwei Punkte auslassen: diejenigen, die entstehen, wenn man $A$ bzw. $B$ verschiebt, weil das Dreieck dann entartet wäre. Das wollen wir dieses Mal aber der Schönheit der Lösung wegen zulassen.)

L 3.4 Es lassen sich maximal jeweils acht Damen, acht Türme (wie gesagt), 14 Läufer, 16 Könige und 32 Springer so aufstellen, dass sie sich nicht gegenseitig bedrohen.

Dass sie sich so aufstellen lassen, sieht man an den unten folgenden Darstellungen sowie an der in der Aufgabenstellung.

Zur vollständigen Bearbeitung der Aufgabe gehört allerdings auch noch zu zeigen, dass dies jeweils die maximal mögliche Zahl ist. Bei den Türmen und Damen ist dies recht einfach: Jede dieser Figuren bedroht eine komplette Spalte, in jeder Spalte darf daher maximal eine Figur stehen, und es gibt genau acht Spalten, also kann man maximal acht dieser Figuren unterbringen.

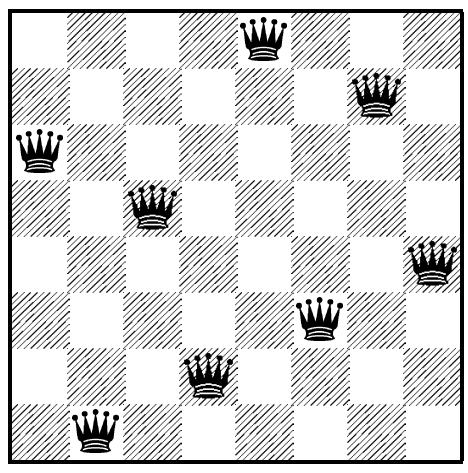

8 Damen ohne Bedrohung

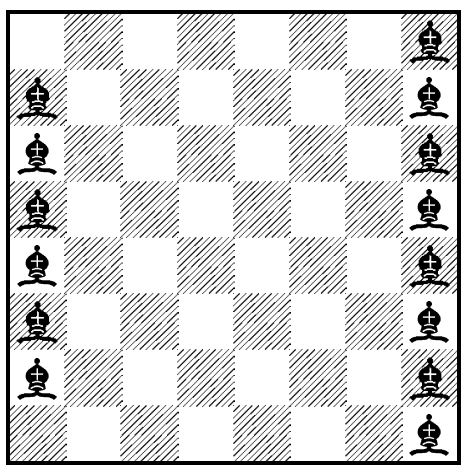

14 Läufer ohne Bedrohung

Bei den Läufern muss man die Diagonalen einer Richtung betrachten, zum Beispiel die Diagonalen von links unten nach rechts oben. Davon gibt es genau 15 (die beiden, die aus nur einem Feld bestehen, müssen mitgezählt werden). Jeder Läufer bedroht in jeder Richtung genau eine Diagonale komplett. Das heißt schon einmal, dass man nicht mehr als 15 Läufer unterbringen kann. Nun ist es aber so, dass ein Läufer, der auf einer der äußeren (nur ein Feld langen) Diagonalen steht, über die lange Diagonale in der anderen Richtung auch die andere äußere Diagonale vollständig 
bedroht. Daher kann man maximal 14 Läufer so auf dem Brett unterbringen, dass sie sich nicht gegenseitig bedrohen.

Um sich die Situation bei den Königen klar zu machen, denke man sich das Brett in 16 Teilbretter der Größe $2 \times 2$ unterteilt. Auf jedem dieser Teilbretter kann maximal ein König stehen, da er dann alle restlichen drei Felder bedroht, also können insgesamt höchstens 16 Könige auf dem Schachbrett stehen.

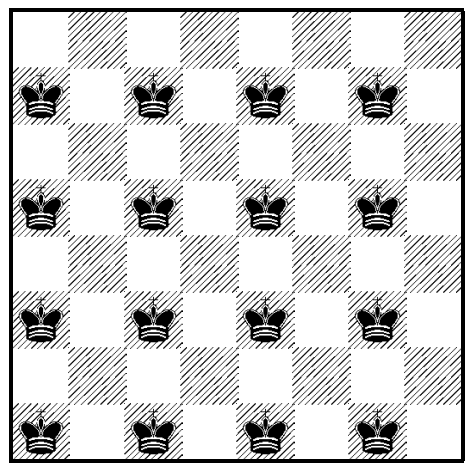

16 Könige ohne Bedrohung

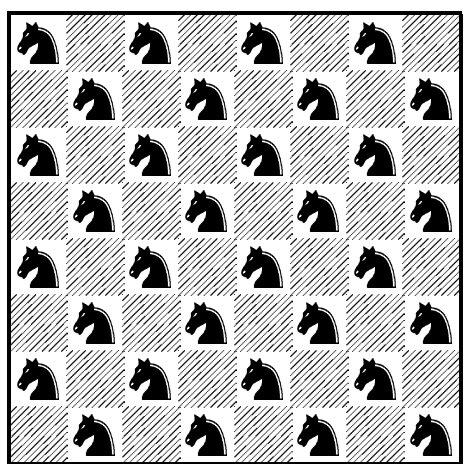

32 Springer ohne Bedrohung

Am trickreichsten ist der Nachweis bei den Springern, obwohl es recht einfach ist festzustellen, dass sich die 32 gezeigten Springer nicht bedrohen, da sie alle nur Felder der anderen Farbe bedrohen. Man denke sich das Schachbrett in acht Teilbretter der Größe $4 \times 2$ unterteilt und betrachte die mit A, B, C und D bezeichneten Paare von Feldern.

$$
\begin{array}{|l|l|l|l|}
\hline \mathbf{A} & \mathbf{C} & \mathbf{B} & \mathbf{D} \\
\hline \mathbf{B} & \mathbf{D} & \mathbf{A} & \mathbf{C} \\
\hline
\end{array}
$$

Sie sind jeweils auf „Rösselsprung-Abstand“, und daher kann jeweils nur ein Springer auf einem Paar von Feldern stehen, insgesamt also nicht mehr als 32. 


\section{Lösungen zu Aufgabenblatt 4}

L 4.1 1. Fall: Genau einer der vier hat die Wahrheit gesagt:

Wir gehen von den letzten beiden Aussagen aus. Carlo und Ede widersprechen sich, d. h. wenn Carlo die Wahrheit sagt, so lügt Ede und umgekehrt. Da es insgesamt nur eine wahre Aussage gibt, ist sie unter diesen beiden und Benno lügt. Also kann höchstens Benno der Täter sein.

Damit haben wir aber noch nicht alle Informationen ausgewertet und wissen somit noch nicht, ob das Problem überhaupt eine Lösung besitzt. Deshalb machen wir eine Probe: Wenn Benno der Täter ist, so lügen Alfred, Benno und Carlo. Ede sagt dann als einziger die Wahrheit.

2. Fall: Genau einer der vier hat gelogen:

Da Carlo und Ede sich widersprechen, sagt genau einer von ihnen die Wahrheit und der andere lügt. Da es insgesamt nur einen Lügner gibt, sagen die anderen beiden die Wahrheit. Also kann nur Carlo der Bösewicht sein.

Auch hier muss wieder eine Probe gemacht werden: Wenn Carlo der Bösewicht ist, so sagen Alfred, Benno und Ede die Wahrheit, Carlo jedoch lügt.

L 4.2 Bei solchen Aufgaben ist es nützlich, ein sogenanntes Weltliniendiagramm zu erstellen, ähnlich wie auf Blatt 1. Dies ist ein Koordinatensystem, auf dessen $x$-Achse die Zeit und auf dessen $y$-Achse der Ort aufgetragen wird. Der Schluss der Schlange befindet sich gemäß Aufgabenstellung zum Zeitpunkt Start am Ort 0 km, zum Zeitpunkt Ende am Ort $1 \mathrm{~km}$, die entsprechenden Punkte $A$ und $B$ werden ins Diagramm eingezeichnet. Gleichförmige Bewegungen (d.h. solche mit konstanter Geschwindigkeit) entsprechen Geraden im Weltliniendiagramm. Verbindet man also die Punkte $A$ und $B$ im Diagramm, so kann man die Position des Schlusses der Schlange zu jedem Zeitpunkt zwischen Start und Ende ablesen.

Genauso zeichnet man die Punkte $C$ und $D$ ein, die die Position der Spitze der Schlange beschreiben. Nachdem man beide Punkte durch eine Gerade (gleichförmige Bewegung!) verbunden hat, hat das Weltliniendiagramm die Gestalt in Abbildung 4.1 .

Jetzt muss noch die Weltlinie von Tick eingezeichnet werden. Diese wird durch drei Punkte bestimmt: den Punkten $A$ und $B$, bei denen Tick zum selben Zeitpunkt am selben Ort ist wie der Schluss der Schlange, und einem Punkt $U$ auf der Weltlinie der Spitze der Schlange, dessen Position zunächst unbestimmt ist. Die zu $U$ gehörige Zeit wird mit Umkehr bezeichnet, der Ort mit $x$. Man erhält Abbildung 4.2.

Wo genau liegt nun der Punkt $U$ auf $C D$ ? Nun, es gibt noch eine Bedingung: Tick läuft stets mit der (betragsmäßig) gleichen Geschwindigkeit, nur vor und nach der Umkehr in entgegengesetzte Richtungen. Der Anstieg von $A U$ ist somit gleich dem 


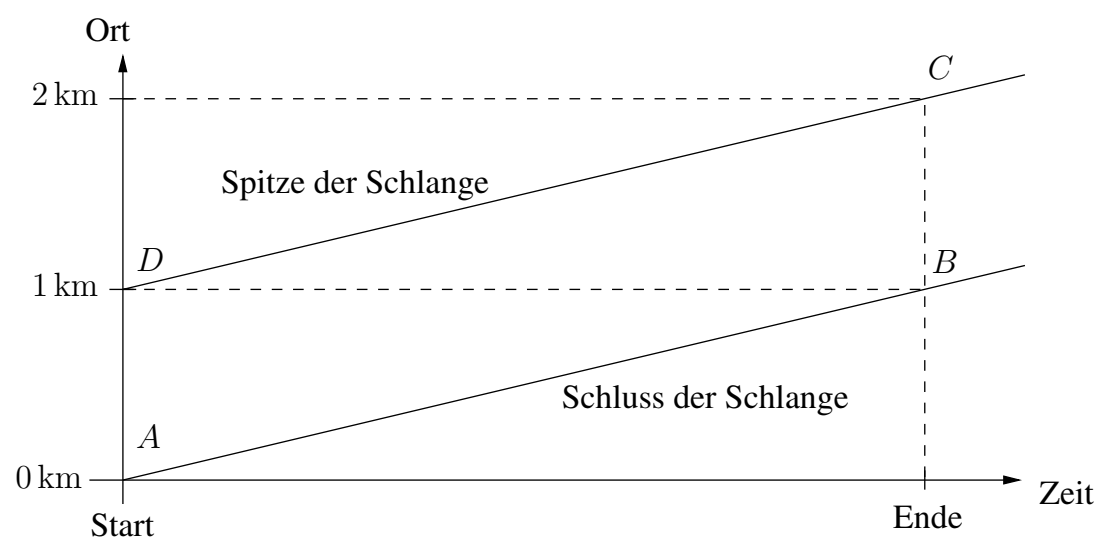

Abbildung 4.1: Weltliniendiagramm mit Spitze und Schluss der Schlange

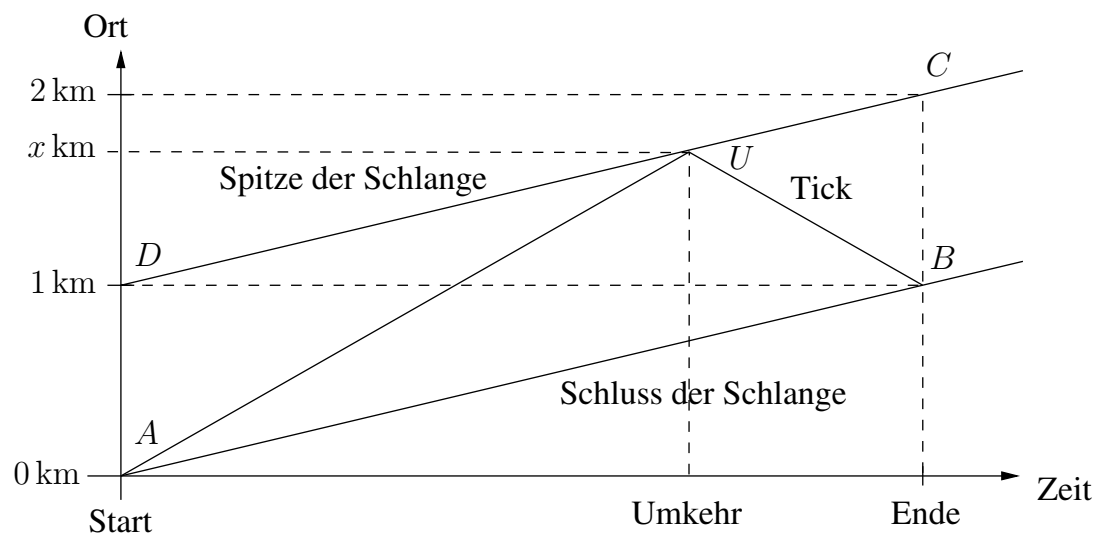

Abbildung 4.2: Weltliniendiagramm mit Schlangenenden und Tick 
negativen Anstieg von $U B$, als Gleichung:

$$
\frac{x}{t_{\text {Umkehr }}-t_{\text {Start }}}=-\frac{-(x-1)}{t_{\text {Ende }}-t_{\text {Umkehr }}}=\frac{x-1}{t_{\text {Ende }}-t_{\text {Umkehr }}} .
$$

Ebenso erhält man aus der Weltlinie der Spitze der Schlange, deren Anstieg vor und nach dem Treffen gleich ist, eine Gleichung:

$$
\frac{x-1}{t_{\text {Umkehr }}-t_{\text {Start }}}=\frac{2-x}{t_{\text {Ende }}-t_{\text {Umkehr }}} .
$$

Setzt man beide Gleichungen zusammen, so erhält man

$$
\frac{x}{x-1}=\frac{t_{\text {Umkehr }}-t_{\text {Start }}}{t_{\text {Ende }}-t_{\text {Umkehr }}}=\frac{x-1}{2-x} \text {. }
$$

Damit erhält man eine quadratische Gleichung für $x$ :

$$
x(2-x)=(x-1)(x-1) \Leftrightarrow 2 x-x^{2}=x^{2}-2 x+1 \Leftrightarrow 0=2 x^{2}-4 x+1
$$

Diese Gleichung hat die zwei Lösungen

$$
x_{1,2}=1 \pm \sqrt{\frac{1}{2}}
$$

wie man dem Diagramm aber entnimmt, liegt $x$ zwischen 1 und 2, also kommt nur die Lösung $x_{1}=1+\frac{1}{\sqrt{2}}$ in Betracht.

Jetzt ist es nicht mehr schwer, Ticks Weglänge $s$ zu bestimmen. Denn es gilt

$$
s=x+(x-1)=2 x-1=2\left(1+\frac{1}{\sqrt{2}}\right)-1=1+\frac{2}{\sqrt{2}}=1+\sqrt{2} \approx 2,41 .
$$

Tick legt also eine Wegstrecke von ca. 2,41 Kilometern zurück.

L 4.3 Verwendet wird der (unter anderem von den Musterlösungen zum letzten Blatt) bekannte Satz vom Sehnenviereck, nach dem die vier Ecken eines Vierecks genau dann auf einem Kreis liegen, wenn gegenüberliegende Innenwinkel zusammen $180^{\circ}$ ergeben.

Sei nun $M$ der Schnittpunkt der Umkreise von Dreieck $A R Q$ und Dreieck BPR. Wenn man zeigen kann, dass $M$ auch auf dem dritten Umkreis liegt, ist die Aufgabe gelöst.

Es gilt aber nach dem eben erwähnten Sehnenvierecksatz:

$$
\alpha+\alpha^{\prime}=180^{\circ}
$$

und

$$
\beta+\beta^{\prime}=180^{\circ}
$$




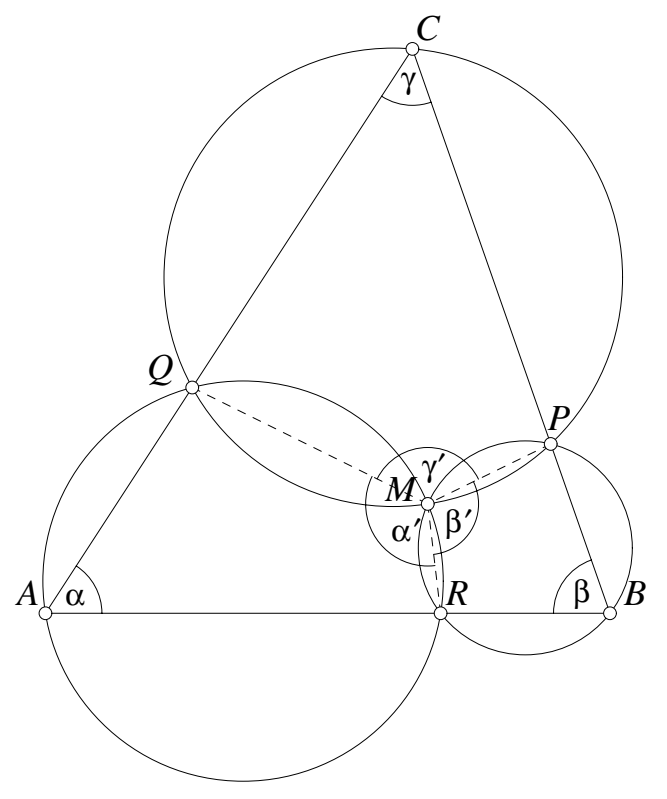

Abbildung 4.3: Skizze zum Beweis der Aufgabe 4.3

Deswegen gilt auch:

$$
\gamma^{\prime}=360^{\circ}-\alpha^{\prime}-\beta^{\prime}=360^{\circ}-\left(180^{\circ}-\alpha\right)-\left(180^{\circ}-\beta\right)=\alpha+\beta .
$$

Weil die Innenwinkelsumme im Dreieck $A B C$ auch $180^{\circ}$ ist, folgt schließlich

$$
\gamma^{\prime}=180^{\circ}-\gamma
$$

Somit ist das Viereck $M P C Q$ ein Sehnenviereck und der Umkreis von $P C Q$ geht auch durch $M$.

L 4.4 Bei dieser Experimentieraufgabe ging es wieder in erster Linie darum, dass man sich ein wenig mit dem Problem beschäftigt und vielleicht ein gewisses Gefühl dafür bekommt, worauf es bei der Aufgabe ankommt. - Abbildung 4.4 zeigt die optimale Einteilung des Kellers: Sie benötigt eine Wandlänge von $\frac{2}{3}+\frac{\pi}{6}+\frac{\sqrt{3}}{4}=$ $1,623278 \ldots$. (wenn die Seitenlänge des Quadrats gleich 1 ist). Wie kommt man auf so eine schöne, aber etwas willkürlich erscheinende Formel für die Länge? Sie ergibt sich aus den Konstruktionsmerkmalen der Figur: Sie ist symmetrisch (womit die mittlere Linie schon einmal ein Geradenstück ist), und die beiden seitlichen Linienzüge sind Kreisbögen, und zwar so, dass sie im rechten Winkel auf die Seitenwand stoßen und 


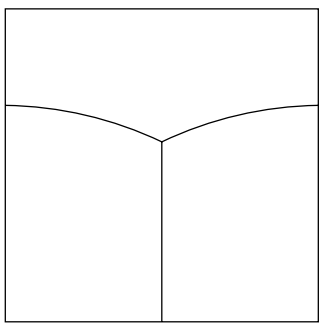

Abbildung 4.4: Die optimale Einteilung

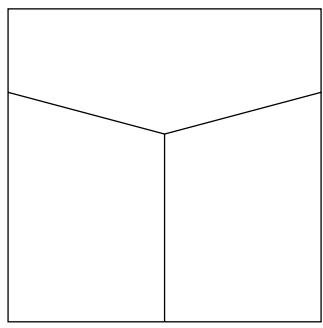

Abbildung 4.5: Die beste Einteilung mit nur geraden Wänden

in der Mitte mit 120-Grad-Winkeln ankommen. Dabei ergibt sich nebenbei gesagt auch, dass der Radius der Kreisbögen genau 1 ist. Übrigens ist erwiesen, dass bei derlei Problemen nur Strecken und Kreisbögen zum Einsatz kommen und dass sie rechtwinklig auf Seitenwände stoßen müssen. Ein allgemeiner Beweis für das 120Grad-Aufeinandertreffen von drei Linienzügen ist uns nicht bekannt, es erscheint aber aus verschiedenen Gründen recht logisch zu sein.

Will man nur gerade Mauern zulassen (etwa weil der Maurer keine Kurven mauern möchte), so ist die Einteilung in Abbildung 4.5 die beste; sie ist wiederum symmetrisch und hat eine Mauerlänge von $\frac{2}{3}+\frac{\sqrt{15}}{4}=1,634912 \ldots$

Das herauszufinden erfordert einigen Aufwand. Vielleicht fällt einem eher die Einteilung in Abbildung 4.6 ein, die mit einer Wandlänge von $1 \frac{2}{3}=1,666 \ldots$ im Vergleich noch ganz gut da steht. Für's Auge hübsch, aber nicht ganz so sparsam sind auch noch die Einteilungen in Abbildung 4.7 mit Gesamtlängen von 1,75 bzw. 2.

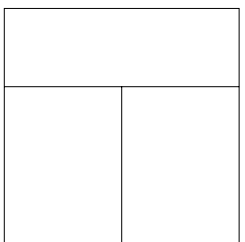

Abbildung 4.6: Noch ganz gut
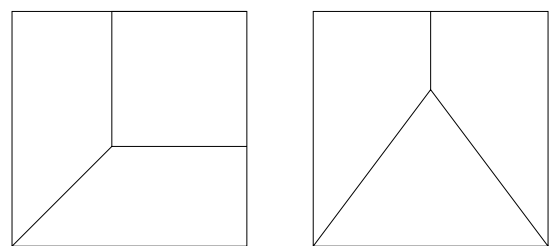

Abbildung 4.7: Auch ganz hübsch 


\section{Lösungen zu Aufgabenblatt 5}

L 5.1 Die zweite Zahl ist die größere.

Wie fast alle bemerkt hatten, kann man eine allgemeinere Aussage beweisen. Es gilt nämlich für alle natürlichen Zahlen $n$ :

$$
\frac{n}{n+3}<\frac{n+1}{n+4} .
$$

Wie beweist man dies?

Dazu gehen wir von einer „offensichtlich“ wahren Aussage aus, die für alle natürlichen Zahlen $n$ gilt:

$$
n+3<n+4 .
$$

Multiplizieren wir beide Seiten mit -1 , so kehrt sich das Relationszeichen um (da $-1<0)$ :

$$
-(n+3)>-(n+4) .
$$

Jetzt dividieren wir beide Seiten durch $n+3$, dabei bleibt das Relationszeichen erhalten, da $n+3>0$ für alle natürlichen Zahlen $n$ gilt:

$$
-1>-\frac{n+4}{n+3} \text {. }
$$

Dann teilen wir beide Seiten durch $n+4$, dabei bleibt das Relationszeichen wieder erhalten, da auch $n+4>0$ :

$$
-\frac{1}{n+4}>-\frac{1}{n+3} .
$$

Wir multiplizieren beide Seiten mit 3:

$$
-\frac{3}{n+4}>-\frac{3}{n+3} .
$$

Nun muss man noch auf beiden Seiten 1 addieren, auch dies ändert an der Relation nichts:

$$
1-\frac{3}{n+4}>1-\frac{3}{n+3} .
$$

Voilà! Sieht man genau hin, so erkennt man darin die gesuchte Ungleichung. Denn nach Erweitern gilt:

$$
\frac{n+4}{n+4}-\frac{3}{n+4}>\frac{n+3}{n+3}-\frac{3}{n+3}
$$

und damit

$$
\frac{n+1}{n+4}>\frac{n}{n+3} \text {. }
$$


Lösungen zu Aufgabenblatt 5

Jetzt setzt man noch $n=7777776$ und hat das gesuchte Ergebnis.

Wer die allgemeinen Umformungen anzweifelt, der kann die Rechnung auch nachvollziehen, indem er überall $n=7777776$ einsetzt, das reicht ja zur Lösung der Aufgabenstellung.

L 5.2 Die natürliche Zahl $n$ habe in Dezimalschreibweise die Ziffern $a_{k}, a_{k-1}, \ldots$ $a_{0}$ in dieser Reihenfolge (wobei $a_{k} \neq 0$ sein soll), so dass also

$$
n=a_{k} \cdot 10^{k}+a_{k-1} \cdot 10^{k-1}+\ldots+a_{1} \cdot 10+a_{0}
$$

gilt. Für die Zahl $m$, die aus $n$ durch Streichen der letzten Ziffer $a_{0}$ und Voranstellen dieser Ziffer entsteht, gilt dann:

$$
m=a_{0} \cdot 10^{k}+a_{k} \cdot 10^{k-1}+\ldots+a_{2} \cdot 10+a_{1}
$$

und die Bedingung der Aufgabe heißt dann $m=4 \cdot n$. Setzt man hier die Darstellungen für $m$ und $n$ ein, so folgt:

$a_{0} \cdot 10^{k}+a_{k} \cdot 10^{k-1}+\ldots+a_{2} \cdot 10+a_{1}=4 \cdot\left(a_{k} \cdot 10^{k}+a_{k-1} \cdot 10^{k-1}+\ldots+a_{1} \cdot 10+a_{0}\right)$

oder umgeformt

$$
\left(10^{k}-4\right) \cdot a_{0}=39 \cdot a_{1}+390 \cdot a_{2}+\ldots+\left(39 \cdot 10^{k-1}\right) \cdot a_{k}
$$

Auf der rechten Seite kann man $39=3 \cdot 13$ ausklammern:

$$
\left(10^{k}-4\right) \cdot a_{0}=3 \cdot 13 \cdot\left(a_{1}+10 \cdot a_{2}+\ldots+10^{k-1} \cdot a_{k}\right)
$$

Da 13 eine Primzahl und $a_{0}$ eine Ziffer zwischen 0 und 9 ist, muss 13 also $10^{k}-4$ teilen. Geht man der Reihe nach die ersten Möglichkeiten für $k$ durch, so findet man, dass 13 keine der Zahlen 6, 96, 996, 9996 teilt. Es gilt aber 13.7692 = 99996=105 -4 . Der kleinstmögliche Wert für $k$ ist also 5 . Setzt man dies in die letzte Gleichung ein, so folgt:

$$
7692 \cdot a_{0}=3 \cdot\left(a_{1}+10 \cdot a_{2}+\ldots+10^{4} \cdot a_{5}\right)
$$

oder, weil $3 \cdot 2564=7692$ ist:

$$
2564 \cdot a_{0}=a_{1}+10 \cdot a_{2}+\ldots+10^{4} \cdot a_{5} .
$$

Der kleinstmögliche Wert für $a_{0}$, für den $2564 \cdot a_{0}$ eine fünfstellige Zahl ist, ist $a_{0}=4$. Damit ist dann

$$
a_{1}+10 \cdot a_{2}+\ldots+10^{4} \cdot a_{5}=4 \cdot 2564=10256 .
$$

Deswegen ist der kleinstmögliche Wert, den ein $n$ mit den geforderten Bedingungen annehmen kann, $n=102564$. 
Die (an dieser Stelle wichtige) Probe zeigt, dass diese Zahl wegen $4 \cdot 102564=410256$ auch wirklich die gesuchte ist.

Ein kleiner Trick kann bei dieser Aufgabe hilfreich sein. Man denke sich nämlich die Zahl $x$ als diejenige rationale Zahl, die die Ziffern unserer gesuchten Zahl $n$ als Periode hat. Also

$$
x=0, a_{k} a_{k-1} \ldots a_{1} a_{0} a_{k} a_{k-1} \ldots a_{1} a_{0} \ldots=0, \overline{a_{k} a_{k-1} \ldots a_{1} a_{0}} .
$$

Dann kann man zunächst

$$
x+a_{0}=a_{0}, a_{k} a_{k-1} \ldots a_{1} a_{0} a_{k} a_{k-1} \ldots a_{1} a_{0} \ldots=a_{0}, \overline{a_{k} a_{k-1} \ldots a_{1} a_{0}}
$$

berechnen. Dividiert man diese Zahl durch 10, so verschiebt sich das Komma um eine Stelle, also:

$$
y:=\frac{1}{10}\left(x+a_{0}\right)=0, a_{0} a_{k} a_{k-1} \ldots a_{1} a_{0} a_{k} a_{k-1} \ldots a_{1} a_{0} \ldots=0, \overline{a_{0} a_{k} a_{k-1} \ldots a_{1}} .
$$

Diese Zahl $y$ hat also die Ziffern der Zahl $m=4 n$ als Periode, so dass nach Bedingung der Aufgabe $y=4 \cdot x$ folgt. Setzt man die Gleichung für $y$ hier ein, so findet man:

$$
\frac{1}{10}\left(x+a_{0}\right)=4 \cdot x
$$

oder umgeformt nach $x$ :

$$
x=\frac{a_{0}}{39} .
$$

Da $n$ nicht mit einer Ziffer 0 beginnen darf, muss die Periode von $x$ auch mit einer von 0 verschiedenen Ziffer beginnen. Die kleinste Möglichkeit hierfür ergibt sich im Fall $a_{0}=4$, nämlich:

$$
x=\frac{4}{39}=0,102564102564102564 \ldots
$$

Dies führt ebenfalls zur Lösung $n=102564$.

L 5.3 Behauptung: Die kleinstmögliche Anzahl an E-Mails, bei der jede der Frauen alles erfährt, ist $2(n-1)$.

Beweis: Eine mögliche Strategie (es gibt auch andere!):

Eine Frau wird zur Chefin gewählt. Am Samstagabend schicken alle anderen Frauen ihre Gewinnmeldung an die Chefin. Nach diesen $n-1$ E-Mails weiß die Chefin alles. Sie schreibt alle Informationen in einer E-Mail zusammen, die sie nacheinander den anderen $n-1$ Frauen schickt. Insgesamt sind dann 2(n-1) E-Mails versendet worden. Da dies aber nur einer von vielen denkbaren Wegen ist, bleibt die Frage: Kommt man auch mit weniger E-Mails aus? 
Wir gehen davon aus, dass keine zwei E-Mails gleichzeitig abgesendet werden. Dann gibt es einen Zeitpunkt $x$, zu dem erstmalig eine Frau alle Informationen erhalten hat. Dazu muss aber jede der anderen ihre Meldung mindestens einmal verschickt haben, d. h. es sind mindestens schon $n-1$ E-Mails verschickt worden. Da aber pro E-Mail jeweils nur eine Frau etwas dazuerfahren kann, muss nach dem Zeitpunkt $x$ jede der anderen $n-1$ Frauen noch mindestens eine weitere E-Mail erhalten.

Also müssen insgesamt mindestens $2(n-1)$ E-Mails verschickt werden.

L 5.4 Allen anders lautenden Behauptungen zum Trotz: Es gibt eine Lösung. Zum Beweis geben wir ganz einfach den entsprechenden Schnitt an. Der rechte Teil ist um den eingezeichneten Punkt um 90 Grad im Uhrzeigersinn zu drehen, dann passt er genau an den linken und es entsteht ein Quadrat.
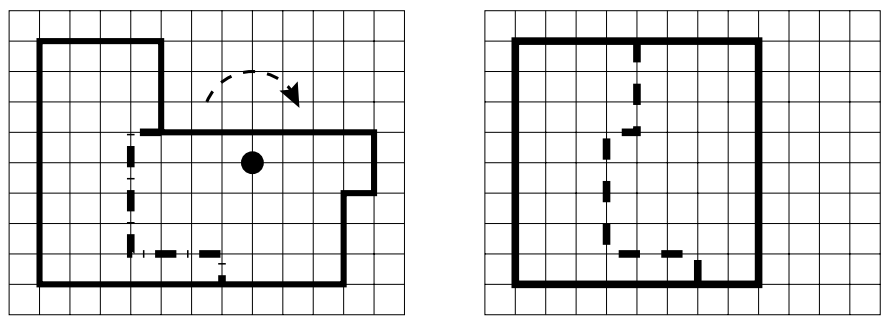

Abbildung 5.1: Schnitt, der die Figur in ein Quadrat überführt

Warum stellen wir eine solche Aufgabe in unserem Mathezirkel? Weil man die Aufgabe nicht nur durch Probieren, sondern auch durch logisches Denken lösen und dabei gleichzeitig beweisen kann, dass es nur diese eine Lösung gibt. (Als Mathematiker möchte man ja immer alles sehr genau wissen ...)

Um es ganz formal zu beginnen: Wir nehmen einmal an, dass es eine Lösung gibt. (Diese formelle Annahme hat einen Vorteil: Sollten wir jetzt nach einigen logischen Folgerungen zu einem Widerspruch kommen, so muss unsere Annahme falsch sein. Und das bedeutet dann, dass wir bewiesen haben, dass es keine Lösung gibt.)

Zunächst schauen wir uns die rechte Spitze der Figur an, in der Abbildung 5.2 mit Ziffer 1 markiert. Sie gehört zu dem einen Teil (er heiße A), den wir durch einen Schnitt aus der Figur erhalten. Da in einem $8 \times 8$-Quadrat keine zwei kleinen Quadrate mehr als sieben Schritte in eine Richtung voneinander entfernt sind, gehören alle mit einer 2 bezeichneten Quadrate zu dem anderen Teil, B. Schaut man sich nun z.B. die linke untere Ecke an, so merkt man entsprechend, dass alle mit 3 bezeichneten Felder zu A gehören.

Da Teil B nun schon acht Felder hoch ist, kann Teil A nur rechts oder links daran angelegt werden. Auf der linken Seite geht das aber nicht, da A dann rechteckig sein müsste. Jetzt kann man sich überlegen, wie A wohl gedreht werden kann, damit es 

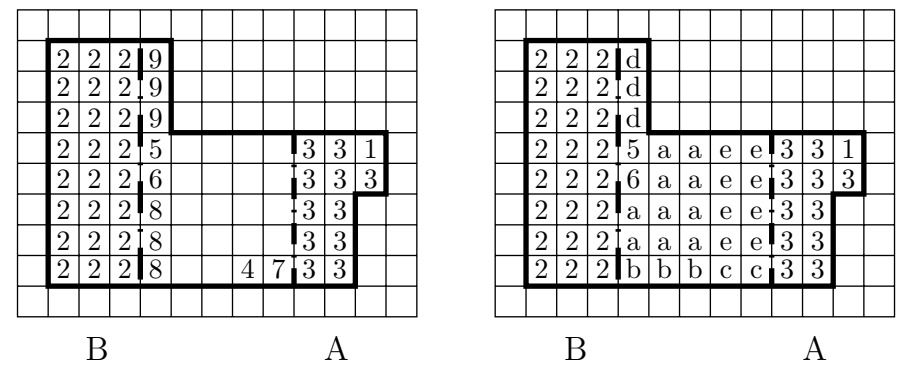

Abbildung 5.2: Bezeichnungen, die im Beweis verwendet werden

an die rechte Seite von B passt. Wir lassen B so liegen, wie es ist. A muss die rechte obere Ecke des Quadrats stellen, weil dort noch nichts ist, deswegen kann es weder nur verschoben werden noch um 90 Grad gegen den Uhrzeigersinn gedreht werden, weil dann die kleine $2 \times 1$-,,Spitze“ über den Rand herausragt.

Soll A um 180 Grad gedreht werden, muss das Feld mit der Nummer 4 die rechte obere Ecke werden, weil einerseits B mindestens drei Felder breit ist und A daher maximal fünf Felder breit sein darf, andererseits ja A im dann oberen Teil mindestens vier Kästchen breit sein muss. Da die Felder 5 und 6 von A überdeckt würden, dürfen sie nicht zu B gehören, machen andererseits damit A zu breit - Widerspruch!

Also wird A um 90 Grad im Uhrzeigersinn gedreht. Jetzt nehmen wir schließlich an, Feld 5 gehöre zu B. Dann kann A in der nach der Drehung rechten Spalte nicht mehr volle acht Kästchen hoch sein; da es aber die rechte obere Ecke belegen muss, muss Feld 7 zu B gehören. Felder 6 und 8 auch, weil sonst A über den oberen Rand hinausragen würde. Felder 9 können nicht mehr zu A gehören, weil sie nur noch an Felder grenzen, die bereits zu B gehören. Damit kann A aber nicht mehr die fünf Kästchen breit sein, die es schon sicher breit ist (Felder 3) - Widerspruch!

Damit muss Feld 5 zu A gehören, das dann die rechte obere Ecke bilden wird. Nun werden langsam die Bezeichnungen unübersichtlich, daher gilt jetzt die rechte Figur. Weil A die 3×4-Lücke oben füllen muss, gehören die Felder 6 und a zu A. Die Felder b können von A nicht bedeckt werden, sie gehören zu B. Weil 5 und 6 in B ,fehlen“, müssen Felder c auch zu A gehören. Schließlich bleiben noch die Felder d bei B und e bei A.

Damit haben wir aus der Annahme, es gebe (irgendeine) Einteilung, genau die oben angegebene Schnittweise hergeleitet, somit also bewiesen, dass es genau diese eine Lösung gibt.

Völlig richtig wurde von einer Teilnehmerin angemerkt, dass es nicht bei jeder Figur mit 64 Kästchen möglich ist, sie mit einem Schnitt in zwei Teile zu zerlegen, die zusammengelegt ein Quadrat ergeben. Das dürfte sogar nur in den wenigsten Fällen machbar sein. Wir haben die Aufgabe in diesem Punkt etwas „vorlaut" formuliert. 


\section{Lösungen zu Aufgabenblatt 6}

L 6.1 Es gibt keine Quadratzahl, deren Quersumme 6 ist.

Dazu erinnern wir uns an die Teilbarkeitsregeln für 3 und 9: Eine Zahl ist genau dann durch 3 teilbar, wenn auch ihre Quersumme durch 3 teilbar ist. Eine Zahl ist genau dann durch 9 teilbar, wenn auch ihre Quersumme durch 9 teilbar ist.

Wir nehmen an, wir hätten eine Quadratzahl $n=m^{2}$ mit Quersumme 6 .

Da die Quersumme 6 durch 3 teilbar ist, ist unsere Zahl $n$ durch 3 teilbar. Weil 3 eine Primzahl ist, muss schon $m$ durch 3 teilbar sein, also kann man mit einer natürlichen Zahl $k$ schreiben: $m=3 k$.

Damit ist $n=m^{2}=(3 k)^{2}=9 k^{2}$ durch 9 teilbar. Dann muss aber auch die Quersumme von $n$ durch 9 teilbar sein. 6 ist nicht durch 9 teilbar, also gibt es keine Quadratzahl mit Quersumme 6.

L 6.2 Die Veranstalter brauchen sich keine Sorgen zu machen! Egal, wie weit die Bäume voneinander entfernt stehen: dort, wo sich die Bänder kreuzen, sind sie 2,40 Meter über der Erde.

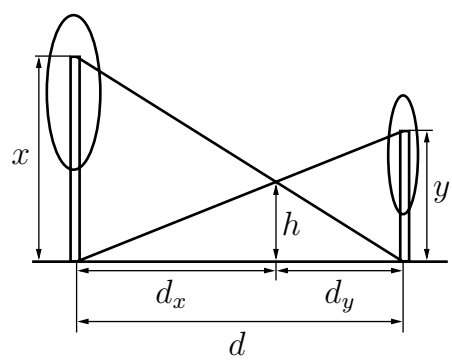

Abbildung 6.1: Skizze zum Beweis der Bänderaufgabe

Zum Beweis benötigt man kaum mehr als die Strahlensätze (nämlich für die Gleichungen (6.1) und (6.2)). Die Skizze definiert die Bezeichnungen für die Lösung. Nun gilt:

$$
\begin{aligned}
\frac{x}{d} & =\frac{h}{d_{y}}, \\
\frac{y}{d} & =\frac{h}{d_{x}} \quad \text { sowie } \\
d_{x}+d_{y} & =d .
\end{aligned}
$$


(6.3) in (6.1) eingesetzt ergibt

$$
\frac{x}{d}=\frac{h}{d-d_{x}},
$$

und (6.2) ist äquivalent zu

$$
d_{x}=\frac{h \cdot d}{y} .
$$

Setzt man dies in (6.4) ein, so erhält man

$$
\begin{aligned}
\frac{x}{d} & =\frac{h}{d-\frac{h \cdot d}{y}}=\frac{h \cdot y}{d \cdot y-d \cdot h} \\
\Leftrightarrow x & =\frac{h \cdot y}{y-h} .
\end{aligned}
$$

Hochmultiplizieren des Nenners

$$
\begin{aligned}
& x y-x h=h y \\
\Leftrightarrow \quad & x y=h(x+y)
\end{aligned}
$$

und Auflösen nach $h$ ergibt

$$
h=\frac{x \cdot y}{x+y} .
$$

Setzt man schließlich die gegebenen Werte ein, bekommt man wie behauptet:

$$
h=\frac{6 \cdot 4}{6+4} \mathrm{~m}=2,4 \mathrm{~m} .
$$

Selbst wenn man davon ausgeht, dass die Schmuckbänder in Wirklichkeit noch ein wenig durchhängen, ist das völlig ausreichend dafür, dass noch ein Mensch darunter durchgehen kann.

L 6.3 Ein (wirklich schlauer!) potentieller Dieb kommt mit nur fünf Versuchen aus.

Der Grund dafür, dass man überhaupt mit weniger als neun Versuchen auskommen kann, ist folgender: Peters ursprünglicher Zahlencode sei $(A, B, C)$, wobei $A, B, C$ jeweils eine der Ziffern 1, 2 oder 3 sind. Stellt der Dieb nun einen Versuchscode $(a, b, c)$ ein und das Schloss öffnet sich, ist er glücklich. Wenn es sich aber nicht öffnet, so kommen auf einen Schlag sieben (!!!) mögliche Codes nicht mehr für $(A, B, C)$ in Frage, nämlich die Codes $(a, b, x),(a, x, c)$ und $(x, b, c)$, wobei $x$ wieder eine beliebige der drei Ziffern 1, 2 oder 3 ist. Wäre nämlich einer dieser Codes gleich $(A, B, C)$, so hätte der Code $(a, b, c)$ mit Peters Zahlencode $(A, B, C)$ wenigstens zwei Ziffern gemeinsam, das defekte Schloss hätte sich also öffnen müssen. 
Wie viele Möglichkeiten hat Peter überhaupt für seinen Code $(A, B, C)$ ?

Für jede der drei Stellen kommen genau drei verschiedene Werte in Frage, also gibt es genau $3^{3}=27$ Möglichkeiten.

Davon kann der Dieb mit nur einem Versuch genau sieben ausschließen!

Insbesondere kann er das Schloss mit drei Versuchen höchstens zufällig, aber nicht sicher (und das war ja gefragt!) knacken, da er bis dahin höchstens $3 \cdot 7=21$ Codes als Peters Zahlencode ausschließen kann. Ist einer der übrigen $27-21=6$ Codes der richtige, so hat sich das Schloss nach den drei Versuchen noch nicht geöffnet.

Aber vier Versuche könnten doch theoretisch reichen, oder?

Schließlich sind $4 \cdot 7=28$ Codes mehr, als es überhaupt gibt, also muss Peters Code dabeigewesen sein, oder?

Nein, leider nicht. Zwar kann der Dieb mit jedem der vier Versuche sieben Codes ausschließen, aber diese müssen nicht alle verschieden sein (und sind es auch nicht). Würde der Dieb zum Beispiel dummerweise viermal denselben Code versuchen, würde er trotzdem nur sieben und nicht $4 \cdot 7=28$ Codes ausschließen. Folgendermaßen sieht man, dass es bei vier Versuchen mindestens zwei solche Überschneidungen gibt, also wenigstens $27-28+2=1$ möglicher Peter-Code unausgeschlossen bleibt:

Für die erste Stelle $a$ eines Codes $(a, b, c)$ hat man drei Möglichkeiten. Unter vier verschiedenen Codes $C_{1}, C_{2}, C_{3}$ und $C_{4}$ muss es also zwei geben, die die gleiche erste Stelle haben. Sei also zum Beispiel $C_{1}=(a, b, c)$ und $C_{2}=(a, d, e)$. Dann sind unter den jeweils sieben Codes, die $C_{1}$ und $C_{2}$ ausschließen, auch die beiden $(a, d, c)$ und $(a, b, e)$, die von beiden ausgeschlossen werden - man hat also mindestens zwei Überschneidungen unter den durch vier Versuche ausgeschlossenen Codes, und es werden insgesamt nicht mehr als $4 \cdot 7-2=26$ Peter-Codes ausgeschlossen. Mit vier Versuchen ist man also auch nicht auf der sicheren Seite, denn es könnte immer noch einer der nicht ausgeschlossenen Codes der richtige sein; dann bliebe das Schloss nach diesen vier Versuchen geschlossen!

Dass es schließlich mit fünf Versuchen sicher klappt, zeigt man zum Beispiel mit folgender Tabelle:

\begin{tabular}{|c|c|l|}
\hline Nr. & Code & Für den richtigen Code dadurch ausgeschlossen: \\
\hline 1 & $(1,1,2)$ & $(1,1,1),(1,1,2),(1,1,3),(1,2,2),(1,3,2),(2,1,2),(3,1,2)$ \\
2 & $(2,2,1)$ & $(2,2,1),(2,2,2),(2,2,3),(2,1,1),(2,3,1),(1,2,1),(3,2,1)$ \\
3 & $(3,3,2)$ & $(3,3,1),(3,3,2),(3,3,3),(3,2,2),(2,3,2)$ \\
4 & $(1,3,3)$ & $(1,3,1),(1,3,3),(1,2,3),(2,3,3)$ \\
5 & $(3,1,3)$ & $(3,1,1),(3,1,3),(3,2,3),(2,1,3)$ \\
\hline
\end{tabular}

Spätestens nach diesen fünf Versuchen ist das Schloss offen, denn in der letzten Spalte der Tabelle kommen alle 27 Möglichkeiten vor. 
Wie man auf die Tabellenlösung kommt? Eine Möglichkeit besteht darin, sich jeden Code als Punkt in einem $3 \times 3 \times 3$-Würfel vorzustellen. Dann schließt jeder Code gerade die Punkte im Würfel aus, die auf einer gemeinsamen achsenparallelen Geraden mit diesem Code liegen.

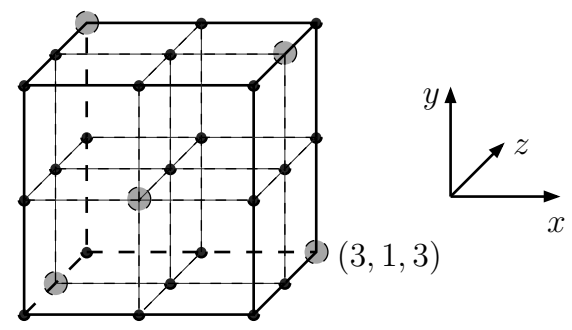

Abbildung 6.2: Die fünf Versuche

In Abbildung 6.2 sind die fünf Codes der Tabelle durch große Punkte markiert. Man erkennt, dass jeder andere Punkt auf einer gemeinsamen achsenparallelen Geraden mit irgendeinem der fünf markierten Punkte liegt. Um solche fünf Punkte zu finden, muss man aber trotzdem noch ein wenig probieren. (Insbesondere wird man, wie zuvor bewiesen, mit vier Punkten nicht auskommen!)

L 6.4 Zunächst einmal eine kleine Geschichte: Am Anfang des zwanzigsten Jahrhunderts gab es noch keine Taschenrechner oder Computer, mit denen Wissenschaftler ihre Berechnungen durchführen konnten. Stattdessen bediente man sich sogenannter Logarithmentafeln; vielleicht habt ihr so etwas schon mal in älteren Formelsammlungen gesehen. Mit diesen konnte man dann auch kompliziertere Berechnungen vereinfachen. Man muss sich das als Buch vorstellen, in dem in einer großen Tabelle für sehr viele Zahlen zwischen 1 und 10 (also z. B. in 1/10000-Schritten) der Logarithmus dieser Zahlen angegeben war, also die Zahl, die man heute beim Taschenrechner durch einfaches Drücken der log-Taste erhält. Wenn ihr das mal ausprobiert, werdet ihr feststellen, dass diese Tabelle ausreicht, um den Logarithmus von allen Zahlen mit einer bestimmten Genauigkeit zu ermitteln (Vergleicht einfach mal die Werte von $\log 1,2, \log 12$ und $\log 120$ !).

Ein amerikanischer Physiker namens Frank Benford, der sein Logarithmentafelbuch sehr oft benutzt haben muss, stellte 1938 fest, dass die vorderen Seiten viel mehr abgegriffen waren als die hinteren. In seinen Berechnungen mussten also sehr viel häufiger Zahlen vorgekommen sein, die mit einer 1 oder 2 angefangen haben, als solche, die mit einer 8 oder 9 beginnen. Diese Feststellung wurde später nach ihm „Benfords Gesetz" oder auch „First-Digit-Gesetz" genannt, obwohl sie auch schon 1881 von Simon Newcomb gefunden wurde:

Die Wahrscheinlichkeit, dass eine Zahl mit 1 beginnt, ist viel größer (ca. 30 Prozent) als die Wahrscheinlichkeit, dass eine Zahl mit 9 beginnt (ca. 
5 Prozent). Genauer gesagt ist die Wahrscheinlichkeit, dass eine Zahl mit der Ziffer $n$ beginnt, $\log \left(\frac{n+1}{n}\right)$ (siehe Schaubild 6.3).

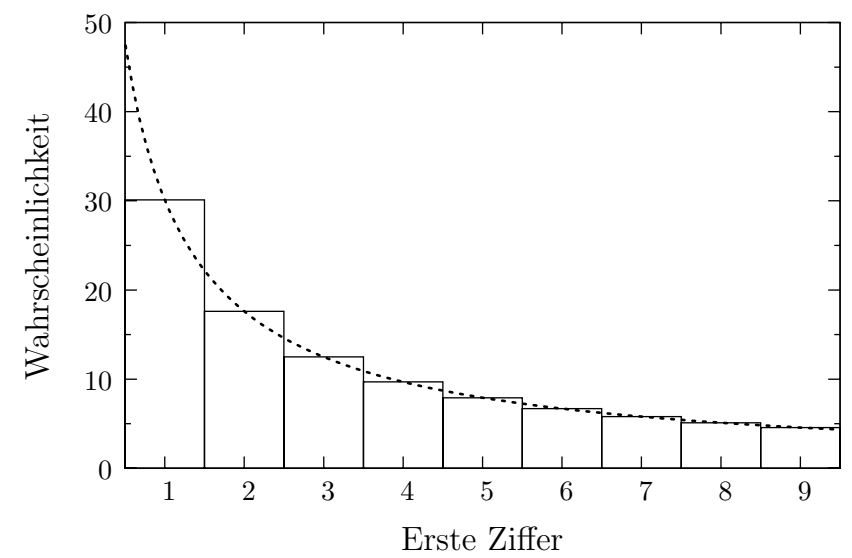

Abbildung 6.3: Von Benfords Gesetz vorausgesagte Verteilung der Anfangsziffern

Kann man dieses „Gesetz" irgendwie begründen?

Die wichtigste Feststellung ist, dass die meisten Vorgänge weniger in absoluten Zahlen als vielmehr in Größenordnungen einzuordnen sind. Es gibt viel mehr Pfützen als Tümpel, mehr Tümpel als Seen und mehr Seen als Meere. Wenn man also die Flächen der Gewässer betrachtet, so gibt es mehr zwischen einem und zwei Quadratmetern als zwischen zwei und drei Quadratmetern, ebenso zwischen 100000 Quadratmetern und 200000 Quadratmetern mehr als zwischen 200000 und 300000. Doch man kann noch genauer analysieren: Die Anzahl der Gewässer mit Größe zum Beispiel zwischen einem und zwei Quadratmetern ist gleich der Anzahl der Gewässer mit Größen zwischen zwei und vier Quadratmetern und der Anzahl mit Größen zwischen vier und acht Quadratmetern. Dies ist die Voraussetzung dafür, dass die Verteilung der Anfangsziffern so aussieht wie im Schaubild angegeben. Denn dann ist der Balken bei der Eins gerade so groß wie die von Zwei und Drei zusammen und so groß wie die von Vier, Fünf, Sechs und Sieben zusammen. Man nennt diese Eigenschaft „Skaleninvarianz".

Solche Verteilungen findet man insbesondere bei exponentiellen Wachstumsvorgängen, was nichts anderes bedeutet, als dass sich eine bestimmte Größe in einem gewissen Zeitintervall (zum Beispiel ein Jahr) jeweils vervielfacht (also zum Beispiel verdoppelt, verdreifacht, halbiert). So etwas findet man in der Umgebung sehr häufig, auf der einen Seite bei Populationen von Bakterien, auf der anderen Seite bei Sparguthaben oder Aktienindizes. Das Erstaunliche ist, dass dieses Gesetz auch bei Größen gilt, für die diese Art von Wachstum nicht so offensichtlich auftritt, zum 
Beispiel bei Dateigrößen auf einer Festplatte oder beliebig ausgewählten Zahlen in einer Zeitung. Es scheint sich also um eine Verteilung der Verteilungen zu handeln! Und wer die Dateigrößen auf seiner Festplatte untersucht hat, wird festgestellt haben, dass diese bei genügend großer Anzahl gut dem Gesetz gehorchen, die Abweichung beträgt meist nur ein paar Prozent. Aber man stellt auch meistens bei der einen oder anderen Zahl einen „Ausreißer" fest. Und solche Ausreißer deuten darauf hin, dass auf dieser Festplatte nicht nur irgendwelche Daten liegen, sondern eine größere Anzahl einer ganz bestimmten Sorte, zum Beispiel Abspeicherungen eines Programms.

Damit hat das Verfahren auch eine praktische Bedeutung: In den USA untersucht man Steuererklärungen großer Unternehmen, indem man die Häufigkeit der Anfangsziffern zählt. Genügen sie Benfords Gesetz, scheint alles in Ordnung. Hat aber jemand die Bilanzen gefälscht, so weicht die Verteilung ab, da die meisten Menschen dazu tendieren, eher Zahlen, die mit 5 oder 6 beginnen, auszuwählen und damit Benfords Gesetz zu verletzen.

Auch bei den von euch untersuchten Supermarktpreisen kann man diese GesetzmäBigkeit feststellen, siehe Schaubild 6.4.

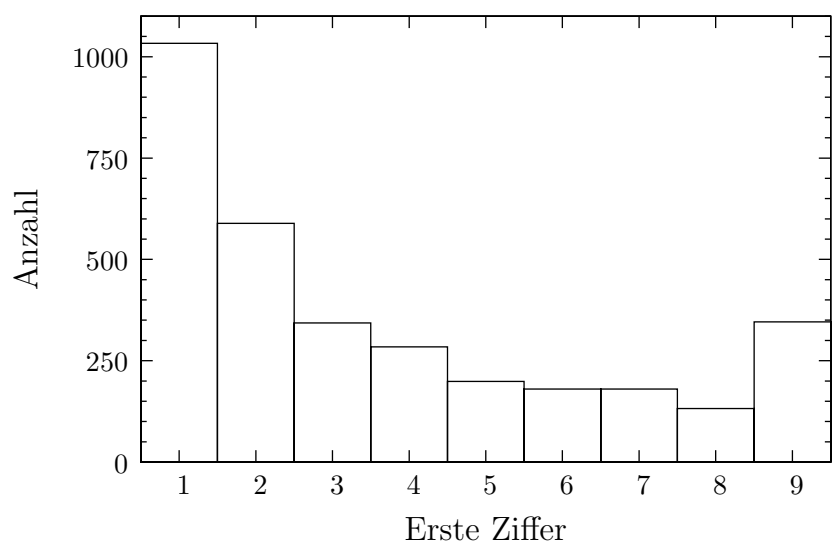

Abbildung 6.4: Verteilung der Anfangsziffern der untersuchten Supermarktpreise

Der Verlauf der Kurve entspricht für die Anfangsziffern 1 bis 8 Benfords Gesetz. Andererseits sieht man deutlich, dass viel mehr Preise, als man nach diesem Gesetz erwarten würde, mit einer 9 beginnen, vermutlich aus psychologischen Gründen. Viel mehr zu diesem Thema findet man im Internet, zum Beispiel unter

- http://de.wikipedia.org/wiki/Gesetz_von_Benford und

- http://www.zmija.de/ziffernanalyse.htm 


\section{Lösungen zu Aufgabenblatt 7}

L 7.1 Wie in der Aufgabe seien $a_{n}$ und $b_{n}$ (mit $a_{n}>b_{n}$ ) die Seitenlängen des DIN-A(n)-Blattes (ohne Angabe der Einheiten).

Die erste Bedingung sagt, dass für alle $n$

$$
\frac{a_{n}}{b_{n}}=\frac{a_{n+1}}{b_{n+1}}
$$

gilt. Die zweite Bedingung lautet

$$
a_{n+1}=b_{n} \quad \text { und } \quad b_{n+1}=\frac{1}{2} a_{n} .
$$

Setzt man (7.2) in (7.1) ein, so erhält man

$$
\frac{a_{n}}{b_{n}}=\frac{a_{n+1}}{b_{n+1}} \stackrel{(7.2)}{=} \frac{b_{n}}{\frac{1}{2} a_{n}}=2 \frac{b_{n}}{a_{n}} .
$$

Multiplizieren mit $a_{n}$ und mit $b_{n}$ liefert

$$
a_{n}^{2}=2 b_{n}^{2} .
$$

Da alle Zahlen positiv sind, liefert Wurzelziehen

$$
a_{n}=\sqrt{2} b_{n} .
$$

Damit ergibt sich für die Seitenverhältnisse

$$
\frac{a_{n}}{b_{n}}=\sqrt{2} .
$$

Mit der dritten Bedingung

$$
a_{0} b_{0}=1
$$

kann man noch die Seitenlängen ausrechnen:

$$
1 \cdot \sqrt{2}=a_{0} b_{0} \sqrt{2}=a_{0} \frac{1}{\sqrt{2}} a_{0} \sqrt{2}=a_{0}^{2},
$$

also

$$
a_{0}=\sqrt[4]{2} \quad \text { und } \quad b_{0}=\frac{1}{a_{0}}=\frac{1}{\sqrt[4]{2}} .
$$

Die Seiten des DIN-A0-Blattes sind also $\sqrt[4]{2} \mathrm{~m} \approx 1,1892 \mathrm{~m}$ und $\frac{1}{\sqrt[4]{2}} \mathrm{~m} \approx 0,8409 \mathrm{~m}$ lang. 
Es gilt außerdem

$$
a_{4} \stackrel{(7.2)}{=} b_{3} \stackrel{(7.2)}{=} \frac{1}{2} a_{2} \stackrel{(7.2)}{=} \frac{1}{2} b_{1} \stackrel{(7.2)}{=} \frac{1}{4} a_{0}=\frac{1}{4} \sqrt[4]{2}
$$

und zusammen mit den ausgerechneten Seitenverhältnissen auch

$$
b_{4}=\frac{1}{\sqrt{2}} a_{4}=\frac{1}{4} \frac{1}{\sqrt{2}} a_{0}=\frac{1}{4} b_{0}=\frac{1}{4} \frac{1}{\sqrt[4]{2}} .
$$

Damit sind die Seiten des DIN-A4-Blattes $\frac{1}{4} \sqrt[4]{2} \mathrm{~m} \approx 29,73 \mathrm{~cm}$ und $\frac{1}{4} \frac{1}{\sqrt[4]{2}} \mathrm{~m} \approx 21,02 \mathrm{~cm}$ lang.

L 7.2 Es seien $a_{1}$ und $a_{2}$ Zahlen, die die Arbeitsleistungen der Bauarbeiter widerspiegeln, und zwar in der Weise, dass $a_{i} \cdot t$ angibt, welchen Anteil des Sandberges Arbeiter Nr. $i$ in der Zeit $t$ wegschaufelt. Wenn $t_{1}$ und $t_{2}$ die Zeit in Stunden darstellen, die die Arbeiter jeweils brauchen, um den Sandberg alleine wegzuschaufeln, gilt also

$$
t_{1} a_{1}=1 \text { und } t_{2} a_{2}=1 .
$$

Nach der zweiten Voraussetzung der Aufgabenstellung gilt, weil beide zusammen den gesamten Haufen in genau 12 Stunden abtragen:

$$
12 \cdot\left(a_{1}+a_{2}\right)=1
$$

Die erste Voraussetzung besagt dann:

$$
\frac{t_{1}}{2}+\frac{t_{2}}{2}=25
$$

Unter Verwendung von (7.4) kann man (7.5) schreiben als

$$
12 \cdot\left(\frac{1}{t_{1}}+\frac{1}{t_{2}}\right)=1
$$

und mit weiteren Umformungen erhält man:

$$
1=12 \cdot\left(\frac{1}{t_{1}}+\frac{1}{t_{2}}\right)=12 \cdot \frac{t_{2}+t_{1}}{t_{1} t_{2}} \stackrel{(7.6)}{=} \frac{600}{t_{1} t_{2}},
$$

also

$$
t_{1} t_{2}=600 \text {. }
$$

Das liefert zusammen mit $(7.6)\left(t_{2}=50-t_{1}\right)$ :

$$
t_{1}^{2}-50 t_{1}+600=0 .
$$


Bisher war in keiner Weise unterschieden, welcher Arbeiter wie schnell schaufelt. Daher müssen die beiden Lösungen der quadratischen Gleichung, nämlich $t_{1}=25 \pm$ $\sqrt{25^{2}-600}=25 \pm 5$, auch schon die beiden gesuchten $t_{i}$ sein; bestimmt man Arbeiter Nr. 1 als den schnelleren, bekommt man demnach:

$$
t_{1}=20 \text { sowie } t_{2}=30 \text {. }
$$

Sie brauchen also 20 bzw. 30 Stunden, wenn sie den Sand alleine wegschaufeln.

L 7.3 Zunächst einmal kann man, wie in der Aufgabe gefordert, feststellen, dass ein auf 12 beginnender 21-Frosch letztlich auf der 3 landet und der 35-Frosch, der auf 11 beginnt, am Ende auf der 1 sitzt.

Nach einer Reihe weiterer Beispiele stellt man schließlich fest, dass der Frosch jedes Mal nach einiger Hüpferei auf einer Zahl sitzen bleibt. Das ist von vornherein keineswegs offensichtlich, schließlich macht der Frosch gelegentlich auch auf dem Zahlenstrahl ziemlich weite Sprünge in Richtung größerer Zahlen. Man muss also erst einmal beweisen, dass der Frosch in jedem Fall irgendwann zur Ruhe kommt. Dies könnte man wie folgt tun:

Man gibt einem $m$-Frosch auf dem Feld $n$ einen gedanklichen Wert $m+n$ und überlegt sich, wie sich dieser Wert des Frosches bei seinem Gehüpfe ändert. Im Fall $m<n$ springt der Frosch nach links und landet auf $n-m$, dann ist sein Wert auf $m+(n-m)=n$ geschrumpft. Ist jedoch $m>n$, so springt der Frosch nach rechts zum Feld $m$, wird dafür aber zum $n$-Frosch; sein Wert bleibt also gleich $m+n$. Im nächsten Schritt allerdings springt er dann als $n$-Frosch nach $m-n$ und sein Wert wird kleiner. Man sieht also: Bei fortwährender Springerei wird der Wert des Frosches wenigstens jeden zweiten Schritt kleiner. Da der Frosch am Anfang nur einen endlichen Wert hat, und der Wert (offensichtlich) nicht negativ werden kann, muss der Frosch nach einiger Zeit aufhören zu springen - was er genau dann tut, wenn $m=n$ ist.

Aber wo hört er auf?

Hier hilft nur, durch viele Beispiele Ideen zu sammeln. Mit der richtigen Idee ist es dann nicht schwer, Folgendes zu beweisen:

Steht ein m-Frosch vor Beginn eines Schrittes auf $n$ und ist nach diesem Schritt zum $m^{\prime}$-Frosch geworden (das kann sich ja geändert haben) und steht auf $n^{\prime}$, so gilt in jedem Fall $\operatorname{ggT}(n, m)=\operatorname{ggT}\left(n^{\prime}, m^{\prime}\right)$.

Hierbei steht $\operatorname{ggT}(a, b)$ für den größten gemeinsamen Teiler der Zahlen $a$ und $b$.

Der Beweis hierzu ist nicht schwer. Ist $m>n$, so tauschen $m$ und $n$ nur die Rollen und es ist klar, dass $\operatorname{ggT}(m, n)=\operatorname{ggT}(n, m)=\operatorname{ggT}\left(m^{\prime}, n^{\prime}\right)$ gilt. Ist $m=n$, so bleibt der Frosch sitzen, und dann ändert sich auch am ggT nichts. Der einzig interessante Fall ist $m<n$. Dann ist zu zeigen, dass $\operatorname{ggT}(m, n)=\operatorname{ggT}(m, n-m)$ gilt. Das gilt aber, denn ist $t$ ein Teiler von $m$ und $n$, so ist $n=n_{1} \cdot t, m=m_{1} \cdot t$, also $n-m=\left(n_{1}-m_{1}\right) \cdot t$. Damit ist $t$ auch ein Teiler von $n-m$. Ist umgekehrt $t$ Teiler 
von $n-m$ und $m$, also $n-m=u \cdot t$ und $m=v \cdot t$, so ist $n=(n-m)+m=(u+v) \cdot t$, folglich teilt $t$ auch $n$. Man sieht daher, dass die gemeinsamen Teiler von $n$ und $m$ genau die gemeinsamen Teiler von $m$ und $n-m$ sind. Also ist auch der größte gemeinsame Teiler derselbe.

Hört der Frosch nun irgendwann als $m$-Frosch auf einem Feld $n$ auf zu springen (und das tut er, wie oben bewiesen!), so ist $n=m$. Ist der Frosch ursprünglich als $a$-Frosch bei $b$ gestartet, so ist dann $\operatorname{ggT}(a, b)=\operatorname{ggT}(m, n)=\operatorname{ggT}(n, n)=n$.

Der Frosch landet also in jedem Fall auf dem Feld $\operatorname{ggT}(a, b)$.

Da 1000000 nur die Primfaktoren 2 und 5 hat, die 123456789 sicherlich nicht hat, landet der von uns betrachtete 1000000 -Frosch, der bei 123456789 startet, letztendlich bei $\operatorname{ggT}(1000000,123456789)=1$.

PS: Vielleicht kennt der eine oder die andere den Euklidischen Algorithmus zur Bestimmung des größten gemeinsamen Teilers zweier natürlicher Zahlen - der Frosch führt im Prinzip genau diesen Algorithmus aus!

L 7.4 Das Problem wurde schon im 18. Jh. von Leonhard Euler unter dem Namen der orthogonalen Lateinischen Quadrate untersucht. Ihm zu Ehren wird heute eine Lösung auch als Eulersches Quadrat bezeichnet.

Eine Möglichkeit, die 16 Karten anzuordnen, ist in der Abbildung 7.1 dargestellt. Man kann leicht überprüfen, dass in keiner Zeile oder Spalte eine Farbe oder ein Bild doppelt vorkommt und dass jede Karte genau einmal verwendet wurde.

Zwar gibt es viele andere mögliche Lösungen, aber sie lassen sich alle auf diese eine zurückführen. Zum einen ist es nämlich so, dass eine Lösung schon eindeutig durch fünf geeignet gewählte Karten festgelegt ist. (Das ist nicht offensichtlich und der Beweis ist auch recht lang, weshalb wir hier darauf verzichten.) Aber daran liegt es, dass man beim Suchen einer Lösung leicht auf Schwierigkeiten stößt, denn wenn man dann bei der sechsten Karte nicht zufällig die richtige legt, kann man keine Anordnung mehr finden und stößt früher oder später auf einen Widerspruch.

Andererseits erhält man aus einer beliebigen Lösung wieder eine Lösung, wenn man Zeilen, Spalten oder auch Farben oder Bilder untereinander vertauscht. Durch diese „Umformungen“ kann man aus einer beliebigen Lösung eine Lösung erzeugen, die mit der Abbildung in den fünf oben gewählten Karten übereinstimmt. (Auch dies soll hier nicht bewiesen werden.) Und dann stimmt sie auch mit der gesamten Abbildung überein, denn es gibt ja, wie oben gesagt, nur eine Lösung mit diesen fünf Karten an diesen Stellen.

Die Aufgabe kann verallgemeinert werden, indem man $n^{2}$ Karten mit $n$ verschiedenen Werten aus einem Spiel mit $n$ verschiedenen Farben nimmt. Damit hat sich Euler beschäftigt und 1779 vermutet, dass es für $n=6$ keine Lösung gibt. Das wurde 1900 bewiesen. Eulers Vermutung, dass es für alle durch 2, aber nicht durch 4 teilbaren Zahlen keine Lösung gibt, war allerdings ziemlich falsch: Seit Mitte des 20. Jh. weiß man, dass es außer für $n=2$ und $n=6$ für alle natürlichen Zahlen $n$ eine Lösung gibt. 
Wenn ihr Lust habt, könnt ihr mal ein Computerprogramm schreiben, das dieses Problem untersucht und zu gegebener Kartenanzahl eine oder alle Lösungen ermittelt.

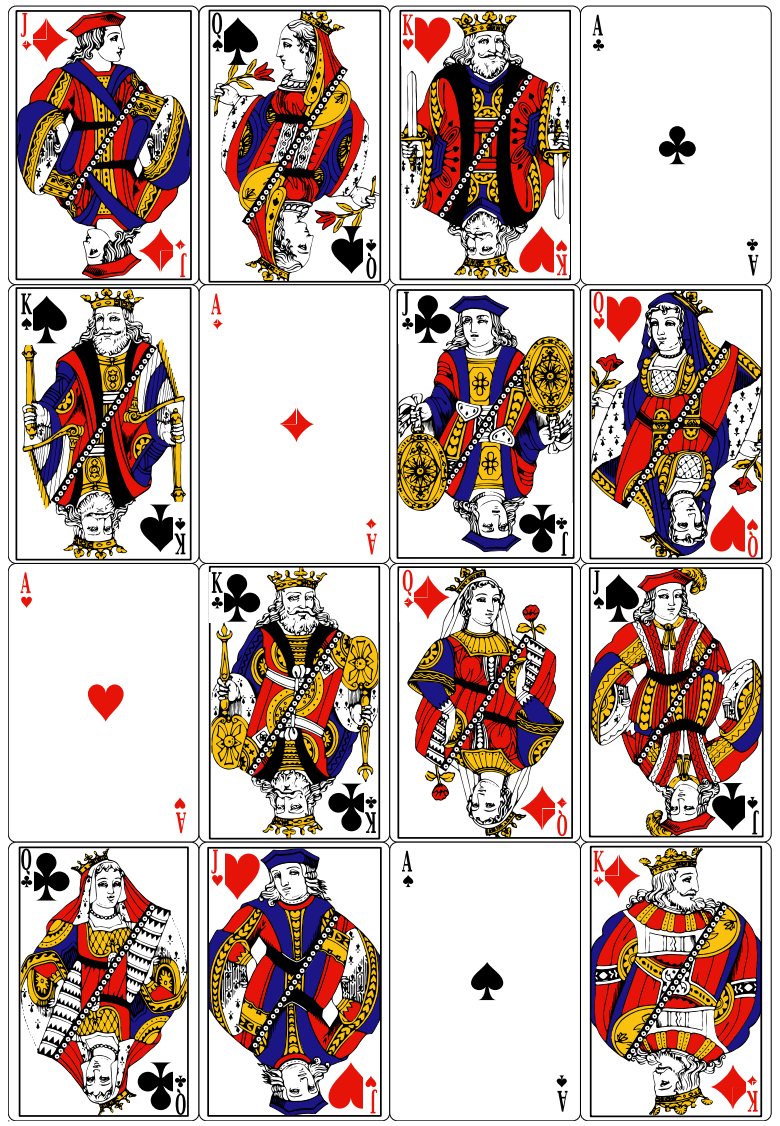

Abbildung 7.1: Das Eulersche Quadrat

(Kartendesign nach David Bellot, http://david.bellot.free.fr/svg-cards/) 


\section{Lösungen zu Aufgabenblatt 8}

L 8.1 Einer der Enkel muss sich verzählt haben. Das kann man folgendermaßen einsehen:

Man zählt die Gesamtsumme $S$ an Geld, die die Oma an den vier Tagen insgesamt verschenkt hat, auf zwei verschiedene Arten.

Wenn der Enkel $i$ insgesamt $a_{i}$ Euro bekommen hat (wobei $1 \leq i \leq 17$ ist), dann gilt einerseits sicherlich

$$
a_{1}+a_{2}+\ldots+a_{17}=S .
$$

Andererseits verschenkt die Oma an jedem der vier Tage insgesamt $1+2+\ldots+17=$ $\frac{1}{2} \cdot(17 \cdot 18)=153$ Euro, an vier Tagen zusammen somit eine Gesamtsumme von

$$
S=4 \cdot 153=612 \text { Euro. }
$$

Damit gilt also

$$
a_{1}+a_{2}+\ldots+a_{17}=612 .
$$

Wären nun alle $a_{i}$ ungerade, so wäre auch die Summe dieser 17 ungeraden Zahlen wieder ungerade, also ungleich 612. Das kann nicht sein.

Bemerkung: In obiger Rechnung wurde die berühmte Gaußsche Summenformel $1+$

\begin{tabular}{|c|c|c|c|c|c|c|c|c|c|c|}
\hline$s$ & $:=$ & 1 & + & 2 & + & $\ldots$ & + & $(n-1)$ & + & $n$ \\
\hline$s$ & $=$ & $n$ & + & $(n-1)$ & + & & + & 2 & + & 1 \\
\hline $2 s$ & $=$ & $(n+1)$ & 1 & $(n+1)$ & + & & & $(n+1)$ & + & $(n+1)$ \\
\hline $2 s$ & $=$ & $n \cdot(n+$ & & & & & & & & \\
\hline
\end{tabular}
$2+\ldots+n=\frac{1}{2} \cdot n \cdot(n+1)$ verwendet. Diese erhält man leicht, wenn man die Summe wie folgt zweimal addiert:

also $s=\frac{1}{2} \cdot n \cdot(n+1)$, wie behauptet.

L 8.2 Wir bezeichnen die Länge der Leiter $\overline{B S}$ mit $l$ und den Abstand $\overline{H S}$ von der hinteren Kante $H$ des Tisches bis zum Punkt $S$, an dem die Leiter an der Wand lehnt, mit $x$ (alle Längenangaben in Metern).

Das Dreieck VHS ist rechtwinklig, also gilt nach dem Satz des Pythagoras:

$$
(\overline{V S})^{2}=(\overline{H S})^{2}+(\overline{V H})^{2}=x^{2}+1 .
$$

Die Tischplatte ist parallel zum Boden, es gilt also $\overline{V H} \| \overline{B E}$, deshalb können wir den Strahlensatz anwenden: 


$$
\frac{\overline{B S}}{\overline{E S}}=\frac{\overline{V S}}{\overline{H S}} .
$$

Setzen wir beide Gleichungen zusammen, so ergibt sich

$$
\frac{(\overline{B S})^{2}}{(\overline{E S})^{2}}=\frac{(\overline{V S})^{2}}{(\overline{H S})^{2}}=\frac{x^{2}+1}{x^{2}} .
$$

Wir multiplizieren die Gleichung mit $(\overline{E S})^{2}$ und erhalten

$$
(\overline{B S})^{2}=\frac{x^{2}+1}{x^{2}} \cdot(\overline{E S})^{2} .
$$

Jetzt setzen wir $\overline{B S}=l$ und $\overline{E S}=x+1$

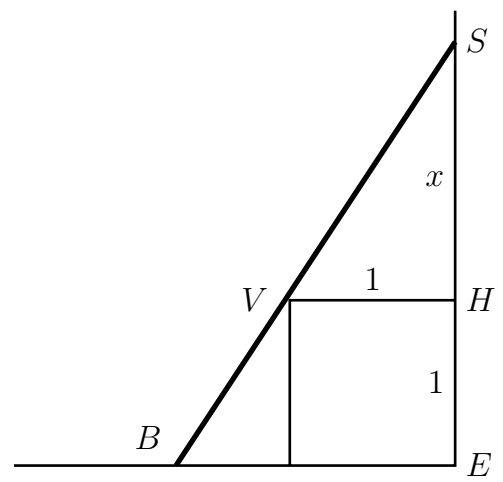
ein:

$$
l^{2}=\frac{x^{2}+1}{x^{2}} \cdot(x+1)^{2}=\frac{x^{2}+1}{x} \cdot \frac{x^{2}+2 \cdot x+1}{x} .
$$

Kürzt man in beiden Brüchen ein $x$, so ergibt sich:

$$
l^{2}=\left(x+\frac{1}{x}\right)\left(x+2+\frac{1}{x}\right) .
$$

Jetzt kommt ein entscheidender Schritt: Wir ersetzen $x+\frac{1}{x}$ durch die neue Variable $u$. Dadurch erhalten wir folgende Gleichung:

$$
l^{2}=u(u+2)
$$

bzw.

$$
u^{2}+2 \cdot u-l^{2}=0 .
$$

Das ist eine quadratische Gleichung in $u$ mit den beiden Lösungen

$$
u_{1,2}=-1 \pm \sqrt{1+l^{2}} .
$$

Wir sind aber eigentlich nicht an dem Ergebnis für $u$ interessiert, sondern an $x$. Deshalb müssen wir die obige Ersetzung nun wieder rückgängig machen.

Aus $u=x+\frac{1}{x}$ folgt durch Multiplikation mit $x$ :

$$
x^{2}-u \cdot x+1=0 .
$$

Das ist wieder eine quadratische Gleichung mit den Lösungen

$$
x_{1,2}=\frac{u}{2} \pm \sqrt{\frac{u^{2}}{4}-1} .
$$


Damit eine reelle Lösung existiert, darf der Ausdruck unter der Wurzel nicht negativ sein, d. h. es muss $u^{2} \geq 4$ gelten. Vorsicht!! Das ist nicht gleichbedeutend mit $u \geq 2$, sondern nur mit $|u| \geq 2$.

Außerdem soll $x$ positiv sein, da die Leiter oberhalb des Tisches an der Wand lehnt. Das geht aber wegen (8.2) nur, wenn $u$ positiv ist, da für $|u| \geq 2$ stets $\left|\frac{u}{2}\right|>\sqrt{\frac{u^{2}}{4}-1}$ gilt. Damit kommt nur die positive Lösung für $u$ in Gleichung (8.1) in Frage.

Weiterhin erhält man aus $u \geq 2$ und Gleichung (8.1) noch eine Bedingung an die Länge der Leiter:

$$
\sqrt{1+l^{2}} \geq 3
$$

Da die Länge der Leiter größer als null ist, folgt daraus

$$
l \geq \sqrt{8}
$$

Gleichheit ist gerade die Situation in Aufgabenteil a). Eine kürzere Leiter kann nicht so angelehnt werden, dass sie Boden, Tisch und Wand gleichzeitig berührt.

Für alle längeren Leitern erhält man durch Gleichung (8.2) zwei mögliche Werte für $x$. Beide sind möglich: Einmal lehnt die Leiter eher flach und einmal eher steil an der Wand. Die Höhe $h$ über dem Boden erhält man durch $h=x+1$.

Konkret ergibt sich für die beiden Aufgabenteile:

a)

$$
u=-1+\sqrt{1+(\sqrt{8})^{2}}=2, \quad x_{1,2}=1 \pm \sqrt{\frac{2^{2}}{4}-1}=1
$$

Die Leiter berührt die Wand 2 Meter über dem Boden.

b)

$$
\begin{gathered}
u=-1+\sqrt{1+6^{2}}=-1+\sqrt{37}, \quad x_{1,2}=\frac{-1+\sqrt{37}}{2} \pm \sqrt{\frac{(-1+\sqrt{37})^{2}}{4}-1} \\
x_{1} \approx 4,88 ; \quad x_{2} \approx 0,20
\end{gathered}
$$

Die Leiter berührt die Wand entweder 5,88 Meter oder 1,20 Meter über dem Boden.

L 8.3 Die Idee zu dieser Lösung lieferte uns Manuel aus Bergfeld.

Seien $r, b$ bzw. $s$ die Anzahl der Züge, bei denen ein roter, blauer bzw. schwarzer Stein hinzukommt. Dann sind am Schluss $17+r-b-s$ rote, $20+b-r-s$ blaue und $24+s-r-b$ schwarze Steine vorhanden. Damit ist die Summe übrig gebliebener blauer und schwarzer Steine $20+b-r-s+24+s-r-b=44-2 r$ eine gerade Zahl. 
Lösungen zu Aufgabenblatt 8

Wenn der letzte Stein schwarz oder blau wäre, so wäre die Summe der blauen und schwarzen Steine $1+0=1$ ungerade. Da dies aber nicht sein kann, muss der letzte Stein ein roter gewesen sein.

Das Folgende war hier zwar nicht gefragt, aber man kann sich noch überlegen, dass ein Spiel tatsächlich so enden kann.

Eine mögliche Zugfolge ist:

\begin{tabular}{l|ccc} 
& BESTAND & Rot & Blau \\
Hinzufügen von & 17 & 20 & 24 \\
\hline \hline Blau & 16 & 21 & 23 \\
Blau & 15 & 22 & 22 \\
\hline 21 Mal Rot & 36 & 1 & 1 \\
\hline Schwarz & 35 & 0 & 2 \\
Blau & 34 & 1 & 1 \\
17 weitere Mal abwechselnd Schwarz und Blau & 0 & 1 & 1 \\
\hline Rot & 1 & 0 & 0
\end{tabular}

L 8.4 Wir können uns unseren Garten als ein Schachbrett vorstellen, dem zwei gegenüberliegende Ecken fehlen. Dazu teilen wir den Garten in $1 \mathrm{~m} \times 1 \mathrm{~m}$ große Quadrate ein und färben (der Natur zuliebe nur in Gedanken) die Quadrate abwechselnd weiß und schwarz ein.

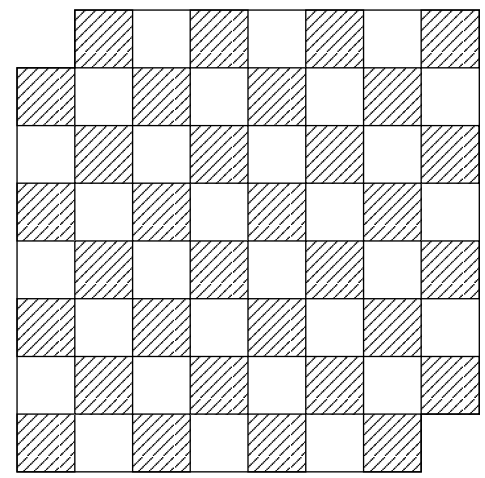

Abbildung 8.1: (Gedankliche) Färbung des Rasens 
Um das linke untere kleine Quadrat vollständig zu bedecken, muss es eine Rasensode geben, deren eine Ecke mit der linken unteren Gartenecke übereinstimmt. Damit kommen auch zwei Kanten der Sode auf Gartengrenzen zu liegen. So mit jeder neu entstandenen Ecke fortfahrend, erhält man:

Will man den Garten vollständig auslegen, so muss jede der Soden genau zwei der kleinen nebeneinander liegenden Quadrate bedecken, also genau ein weißes und genau ein schwarzes.

Könnte man den Garten vollständig mit den 31 Soden auslegen, so wären 31 schwarze und 31 weiße kleine Quadrate verdeckt.

Bei unserer Färbung besteht der Garten aber aus 32 kleinen Quadraten in Schwarz und 30 in Weiß, die Verteilung der Grassoden im Garten ist also ohne Zurechtschneiden nicht möglich. 


\section{Lösungen zu Aufgabenblatt 9}

L 9.1 Um alle Zeitpunkte, an denen eine solche Verwechslung möglich ist, exakt zu bestimmen, muss man wohl oder übel ein wenig rechnen. Zunächst einmal bezeichne $m$ die Position des Minutenzeigers, wobei $0 \leq m<60$ sei, und genauso sei $s$ die Position des Stundenzeigers mit $0 \leq s<12$. (Es reicht offensichtlich, die erste Tageshälfte zu betrachten, da sich die Situation in der zweiten exakt wiederholt.) Welche Bedingung muss man an $m$ und $s$ stellen, damit sich eine sinnvolle Uhrzeit ergibt? (Zum Beispiel wäre $m=10$ und $s=0$ sicherlich unsinnig, weil Punkt zwölf Uhr auch der Minutenzeiger bei $m=0$ stehen muss.)

Das Problem ist, dass allein der Stundenzeiger die genaue Uhrzeit bestimmt. Wenn er zum Beispiel genau auf einer vollen Stunde steht, muss $m=0$ sein, wenn er genau zwischen zwei vollen Stunden steht, ist $m=30$ usw. In Formeln muss man also zunächst den Bruchteil der angefangenen Stunde berechnen, den der Stundenzeiger anzeigt, und das Ergebnis dann mit 60 multiplizieren, was die aktuellen Minuten ergeben muss. Also:

$$
m=60 \cdot(s-\lfloor s\rfloor) .
$$

$(\lfloor$.$\rfloor ist die sogenannte Gaußklammer. Bei positiven Zahlen tut sie nichts weiter, als$ alle Nachkommastellen zu streichen, wie z. B. $\lfloor 2,31\rfloor=2,\lfloor 9,9\rfloor=9$ oder $\lfloor 6\rfloor=6$.) Interpretiert man nun versehentlich die Zeigerbedeutung um, so entspricht die Stellung $m$ der Minuten offenbar der Stellung $s^{\prime}=\frac{m}{5}$ eines Stundenzeigers und außerdem die Stellung $s$ des Stundenzeigers der Stellung $m^{\prime}=5 \cdot s$ eines Minutenzeigers. Damit auch diese Stellung sinnvoll ist, muss analog

$$
m^{\prime}=60 \cdot\left(s^{\prime}-\left\lfloor s^{\prime}\right\rfloor\right)
$$

gelten. Also

$$
5 \cdot s=60 \cdot\left(\frac{m}{5}-\left\lfloor\frac{m}{5}\right\rfloor\right) .
$$

Setzt man hierin noch (9.1) ein, so erhält man die gesuchte Bedingung an die Stellung $s$ des großen Zeigers:

$$
s=12 \cdot(12 \cdot(s-\lfloor s\rfloor)-\lfloor 12 \cdot(s-\lfloor s\rfloor)\rfloor)
$$

Dies entspricht der Gleichung

$$
\begin{aligned}
s= & 144 s-144\lfloor s\rfloor-12 \cdot\lfloor 12 s-12\lfloor s\rfloor\rfloor \\
= & 144 s-144\lfloor s\rfloor-12 \cdot(\lfloor 12 s\rfloor-12\lfloor s\rfloor) \\
& \quad(\text { weil } 12\lfloor s\rfloor \text { eine ganze Zahl ist) } \\
= & 144 s-12\lfloor 12 s\rfloor
\end{aligned}
$$


und es gilt „nur“ noch, deren Lösungen unter der Einschränkung $0 \leq s<12$ zu finden. Eben wegen jener Einschränkung gibt es aber genau eine natürliche Zahl $k$ mit $0 \leq k<144$ so, dass $\frac{k}{12} \leq s<\frac{k+1}{12}$ ist. (Wenn man die gesamten zwölf Stunden in Fünf-Minuten-Intervalle unterteilt, so gibt $k$ gerade die Nummer des Intervalls an, in welches die Uhrzeit fällt, wobei man bei 0 anfangen muss zu zählen.) Der Vorteil dieses $k$ ist dann gerade, dass $\lfloor 12 s\rfloor=k$ gilt. Setzt man dies in die letzte Gleichung ein, so folgt:

$$
s=\frac{12}{143} \cdot k \text {. }
$$

Diese Gleichung liefert nun für jedes $k \in\{0,1, \ldots, 142\}$ tatsächlich einen Wert $s$, für den die Bedingung $\frac{k}{12} \leq s<\frac{k+1}{12}$ gilt (für $k=143$ ist das nicht der Fall, also ergibt sich hier keine Lösung). Man hat also in zwölf Stunden genau 143 Zeitpunkte, an denen man die beiden Zeiger „mit Sinn“ vertauschen könnte, an einem ganzen Tag geht das also 286-mal. („24 Uhr“ zählt schon zum nächsten Tag!) Für $s$ sind das die gerundeten Dezimalzahlen $0 ; 0,083 ; 0,167$; usw. und in der gewohnten Uhrzeitangabe entspricht dies etwa 00.00.00 Uhr, 00.05.02 Uhr, 00.10.04 Uhr usw.

L 9.2 Am einfachsten kommt man zur Lösung, wenn man feststellt, dass der angegebene Term dem Produkt $(n+1)^{4}=n^{4}+4 n^{3}+6 n^{2}+4 n+1$ ähnelt. Dann ergibt sich mit der dritten binomischen Formel:

$$
\begin{aligned}
4 n^{3}+6 n^{2}+4 n+1 & =(n+1)^{4}-n^{4} \\
& =\left((n+1)^{2}+n^{2}\right)\left((n+1)^{2}-n^{2}\right) \\
& =\left((n+1)^{2}+n^{2}\right)(2 n+1) .
\end{aligned}
$$

Man hat also eine Zerlegung der Zahl in zwei Faktoren, wobei offensichtlich der zweite Faktor der kleinere ist. Für $n \geq 1$ ist dieser Faktor größer als eins, also hat man zwei echte Teiler der Zahl gefunden, die demnach nicht prim ist. Wenn null auch zu den natürlichen Zahlen gezählt werden soll (die Gelehrten streiten sich darüber), hat man ebenfalls keine Probleme: Für $n=0$ ist der Term gleich eins, und das ist nach Definition keine Primzahl.

L 9.3 Zunächst die nackten Zahlen: Die sieben Zwerge können ohne Wiederholung der Reihenfolge 544 Tage zur Arbeit gehen, mit Schneewittchen sind es 2770 Tage.

Nun der Lösungsweg: Die Hinzunahme von Schneewittchen ist - mathematisch betrachtet - nichts anderes, als hätte man acht verschieden große Zwerge statt sieben. Für sieben Zwerge kann man die Anzahl der verschiedenen Möglichkeiten mit etwas Geduld noch per Hand auszählen, indem man einige Fallunterscheidungen macht und z. B. ausnutzt, dass der kleinste Zwerg logischerweise ja an einer „Tief“-Position stehen muss und der zweitkleinste eine „Hoch“-Position allenfalls an einem Ende der Schlange einnehmen kann. Das bedeutet aber schon einige Arbeit, und mit acht Personen ist das ohne massive Zuhilfenahme von Notizen auf Papier wohl nicht mehr 
möglich. Einfacher ist es da, sich Schritt für Schritt an die Lösung(en) heranzutasten, indem man sich die Zahlen rekursiv herleitet, angefangen mit ganz wenigen Zwergen.

Dazu seien folgende Bezeichnungen eingeführt: $H_{n}$ stehe für die Anzahl an Möglichkeiten, eine Zwergenreihe aus $n$ Zwergen so zu bilden, dass vorne eine "Hoch"Position ist (das heiße eine $H$-Reihe), $T_{n}$ entsprechend für die Anzahl mit einer "Tief"-Position vorne ( $T$-Reihe). Eine kurze Überlegung zeigt, dass immer $H_{n}=T_{n}$ gelten muss, denn indem man den größten Zwerg durch den kleinsten ersetzt, den zweitgrößten durch den zweitkleinsten usw., kann man jeder $H$-Reihe genau eine $T$-Reihe zuordnen und umgekehrt.

Mit nur einem Zwerg kann man lediglich genau eine Reihenfolge bilden, die sowohl eine $H$ - als auch eine $T$-Reihe ist $\left(H_{1}=T_{1}=1\right)$. Bei zwei Zwergen hat man für eine $H$-Reihe auch keine Wahlmöglichkeit: der größere der beiden muss nach vorne. Also: $H_{2}=T_{2}=1$. Ab drei Zwergen wird es etwas interessanter: Für eine $H$-Reihe muss der kleinste Zwerg in die Mitte - aber die anderen beiden können wählen, wer vorne und wer hinten geht. Das macht $H_{3}=2$.

$\mathrm{Ab}$ vier Zwergen soll nun anders vorgegangen werden: Wir betrachten speziell den größten Zwerg. In einer $H$-Reihe kann er an erster oder dritter Stelle gehen (oder, wenn es mehr Zwerge sind, an fünfter, siebter, ... Stelle). Bei der Aufteilung der anderen Zwerge auf zwei Gruppen, die vor bzw. hinter dem größten Zwerg gehen, muss man sich zuerst überlegen, wie viele Möglichkeiten es dafür gibt. (Die genaue Reihenfolge der anderen Zwerge soll noch nicht beachtet werden.) Das kann man bei den relativ kleinen Zahlen, um die es hier geht, zur Not auch noch von Hand machen. Besser ist es aber auch hier mit einer Systematik. (Einige von euch werden sicherlich schon mit Binomialkoeffizienten gearbeitet haben, sie können die nachfolgenden Erklärungen ggf. überspringen.) Für die Gruppe von $n-1$ übrig bleibenden Zwergen muss also ermittelt werden, wie viele Möglichkeiten es gibt, $k$ Zwerge als "Vorweggehende“ auszuzeichnen. (Dass die anderen dann hinten gehen, ergibt sich natürlich von selbst.) Für die erste „Auszeichnung“ hat man sicher $n-1$ Möglichkeiten, für die zweite $n-2$ usw. Das macht $(n-1)(n-2) \cdot \ldots \cdot(n-k)$ Varianten. Da aber die Reihenfolge der „Auszeichnungen“ hier ohne Bedeutung ist, muss man noch herausdividieren, wie viele Möglichkeiten es für eine bestimmte Gruppe gibt, ausgezeichnet worden zu sein (bzgl. der Reihenfolge). In einer Gruppe von $k$ Leuten gibt es (gleiches Verfahren) $k$ Möglichkeiten für das Mitglied, das als erstes ausgezeichnet wurde, $k-1$ für das zweite usw. Das heißt also, dass immer $k(k-1) \cdot \ldots \cdot 1$ Auszeichnungsreihenfolgen genau zur gleichen Gruppenbildung führen. Insgesamt gibt es also $\frac{(n-1)(n-2) \cdot \ldots \cdot(n-k)}{k(k-1) \cdot \ldots \cdot 1}$ Möglichkeiten, eine Teilmenge von $k$ Zwergen aus $n-1$ Zwergen zu bilden. (Der eben genannte Bruch ist ein so genannter Binomialkoeffizient, aber mehr Erklärungen dazu würden an dieser Stelle zu weit führen.)

Damit die gesamte Zwergenreihe eine $H$-Reihe wird, müssen die $k$ Zwerge, die vor dem größten Zwerg gehen, natürlich selbst eine $H$-Reihe bilden (wofür es bekanntlich 
$H_{k}$ Varianten gibt). Der größte Zwerg muss an einer ungeraden Position gehen und die $n-1-k$ Zwerge, die hinter dem größten gehen, müssen eine $T$-Reihe bilden ( $T_{n-1-k}$ Möglichkeiten). Das heißt also, dass man jetzt, wenn man die $H_{k}$ und $T_{k}$ für $k \leq n$ kennt, $H_{n+1}$ (und damit auch $T_{n+1}$ ) berechnen kann. Und genau das machen wir nun. (Wenn der größte Zwerg ganz vorne oder hinten geht, braucht man sich um die Aufteilung der anderen Zwerge nach vorne und nach hinten natürlich keine Gedanken zu machen, da gibt es nur eine Möglichkeit.)

Bekannt ist: $H_{1}=T_{1}=1, \quad H_{2}=T_{2}=1, \quad H_{3}=T_{3}=2$. Also ist

$$
\begin{aligned}
H_{4} & =T_{3}+\frac{3 \cdot 2}{2 \cdot 1} H_{2} T_{1}=2+3 \cdot 1 \cdot 1=5 \\
H_{5} & =T_{4}+\frac{4 \cdot 3}{2 \cdot 1} H_{2} T_{2}+H_{4}=5+6 \cdot 1 \cdot 1+5=16 \\
H_{6} & =T_{5}+\frac{5 \cdot 4}{2 \cdot 1} H_{2} T_{3}+\frac{5 \cdot 4 \cdot 3 \cdot 2}{4 \cdot 3 \cdot 2 \cdot 1} H_{4} T_{1}=16+10 \cdot 1 \cdot 2+5 \cdot 5 \cdot 1=61 \\
H_{7} & =T_{6}+\frac{6 \cdot 5}{2 \cdot 1} H_{2} T_{4}+\frac{6 \cdot 5 \cdot 4 \cdot 3}{4 \cdot 3 \cdot 2 \cdot 1} H_{4} T_{2}+H_{6} \\
& =61+15 \cdot 1 \cdot 5+15 \cdot 5 \cdot 1+61=272 \\
H_{8} & =T_{7}+\frac{7 \cdot 6}{2 \cdot 1} H_{2} T_{5}+\frac{7 \cdot 6 \cdot 5 \cdot 4}{4 \cdot 3 \cdot 2 \cdot 1} H_{4} T_{3}+\frac{7 \cdot 6 \cdot 5 \cdot 4 \cdot 3 \cdot 2}{6 \cdot 5 \cdot 4 \cdot 3 \cdot 2 \cdot 1} H_{6} T_{1} \\
& =272+21 \cdot 1 \cdot 16+35 \cdot 5 \cdot 2+7 \cdot 61 \cdot 1=1385
\end{aligned}
$$

Damit gibt es für die sieben Zwerge $H_{7}+T_{7}=2 \cdot H_{7}=544$ und für die Zwerge mit Schneewittchen $2 \cdot H_{8}=2770$ verschiedene Möglichkeiten, auf Arbeit zu gehen.

L 9.4 Mit ein wenig Probieren findet man schnell die richtige Idee, wie man die Ebene auch mit allgemeinen Vierecken pflastern kann. Sie ist in der folgenden Abbildung dargestellt:

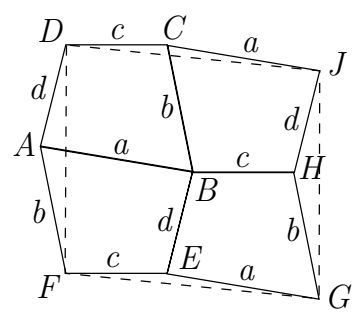

Abbildung 9.1: Die Pflasterung

Hierbei wird nichts weiter getan, als dass irgendein schon vorhandenes Viereck an irgendeiner der Mittelpunkte der vier Seiten um $180^{\circ}$ gedreht wird. So entsteht zum Beispiel das Viereck $B A F E$ aus dem Viereck $A B C D$ durch eine $180^{\circ}$-Drehung um den Mittelpunkt der Seite $A B$.

Aber warum funktioniert dieses Verfahren und füllt die Ebene lückenlos und ohne Überschneidungen? 
Wenn man bei der Erklärung ein wenig auf Exaktheit verzichtet, so kann man anschaulich argumentieren:

Hierzu betrachte man das eben schon erwähnte Beispiel der $180^{\circ}$-Drehung um den Mittelpunkt der Seite $A B$. Dabei wird $B$ auf $A$ und $A$ auf $B$ abgebildet - die Seite $A B$ also insgesamt auf sich selbst. Es wird demnach entlang der Seiten nie irgendwelche Überstände geben.

Und warum schließt sich die Figur exakt, wenn man einmal die vier Vierecke (siehe Abbildung) „im Kreis" gespiegelt hat?

Die Antwort hierauf ist ganz einfach: In jedem Viereck addieren sich die Innenwinkel $\mathrm{zu} 360^{\circ}$, also zu einem Vollwinkel, und wie man sich an der Abbildung leicht überzeugt, treffen bei dem beschriebenen Verfahren stets genau die vier verschiedenen Innenwinkel an einem Punkt aufeinander (im Bild ist dies der Punkt B). Übrigens gelten diese Überlegungen genauso für nicht konvexe Vierecke („eingedellte Drachen“); ein entsprechendes Bildchen könnte wie in Abbildung 9.2 aussehen. Wenn

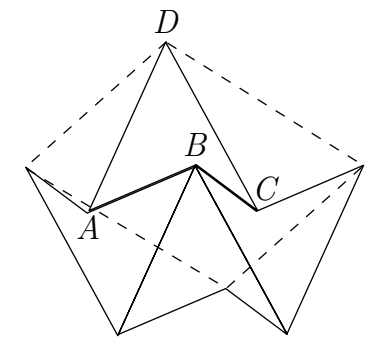

Abbildung 9.2: Die Pflasterung mit „Drachen“

man mehr auf mathematische Exaktheit bedacht ist, kann man den Sachverhalt auch formal beweisen. Das Verfahren hierzu sei aber nur kurz angedeutet:

Man betrachte noch einmal die erste Abbildung. Man erkennt leicht, dass die Dreiecke $A F D$ und $H G J$ kongruent sind, da sie in zwei Seiten ( $b$ und $d$ ) und dem eingeschlossenen Winkel übereinstimmen. Deswegen gilt dann auch, dass die Strecken $F D$ und $G J$ gleich lang sind. Genauso kann man auch zeigen, dass die Strecken $F G$ und $D J$ gleich lang sein müssen, so dass letztendlich das Viereck FGJD ein Parallelogramm ist.

Nun argumentiert man folgendermaßen: Wir wissen schon, dass man mit Parallelogrammen die Ebene lückenlos pflastern kann, also auch mit zum Parallelogramm FGJD kongruenten Parallelogrammen. In dieser Pflasterung kann man dann auch problemlos bei jedem Parallelogramm das Dreieck GJH „herausschneiden“ und als das kongruente Dreieck $F D A$ wieder anfügen. Genauso verfährt man mit den Dreiecken, die zu $F G E$ bzw. DJC kongruent sind.

Auf diese Weise erhält man eine Pflasterung mit den gegebenen Vierecken. 


\section{Lösungen zu Aufgabenblatt 10}

L 10.1 Herr von Schluckspecht befindet sich mit einer Wahrscheinlichkeit von $25 \%$ in der letzten Kneipe.

An jedem Abend, den er nicht zu Hause verbringt, befindet sich Herr von Schluckspecht mit einer Wahrscheinlichkeit von $\frac{3}{4}=\frac{9}{12}$ in einer der neun Kneipen $A, \ldots, I$, die auch seiner Frau bekannt sind. Also befindet er sich mit einer Wahrscheinlichkeit von $\frac{1}{4}=\frac{3}{12}$ woanders. Ohne das Problem zu verändern, kann man annehmen, dass er an den Abenden, die er weder zu Hause noch in einer der Kneipen $A, \ldots, I$ verbringt, einen von drei Orten $X, Y, Z$ aufsucht, von denen er dann auch keinen bevorzugt.

An besagtem Abend befindet sich Herr von Schluckspecht also mit einer Wahrscheinlichkeit von $100 \%$ an einem der Orte $A, \ldots, I, X, Y, Z$. Für jeden der Orte ist die Wahrscheinlichkeit $p$, mit der er sich dort aufhält, gleich groß, nämlich $\frac{1}{12}$.

Herr von Schluckspecht bleibt die ganze Nacht an dem Ort, für den er sich am Abend entschieden hat. Daher weiß seine Frau, nachdem sie die ersten acht Kneipen in alphabetischer Reihenfolge erfolglos durchsucht hat, dass er sich mit einer Wahrscheinlichkeit von $100 \%$ an einem der Orte $I, X, Y$ oder $Z$ befindet. Bevor sie losgegangen war, wusste sie, dass er sich mit einer Wahrscheinlichkeit $q=\frac{1}{3} \quad\left(q=\frac{1}{12}+3 \cdot \frac{1}{12}\right)$ für eine dieser vier Lokalitäten entschieden hatte.

Um nun herauszufinden, mit welcher Wahrscheinlichkeit $r$ er sich in der letzten ihr bekannten Kneipe befindet, muss sie also nur das Verhältnis $r=\frac{1}{\frac{12}{3}}=\frac{1}{4}$ bestimmen. Also befindet er sich mit einer Wahrscheinlichkeit von $25 \%$ in der letzten Kneipe. Hinweis: Herr von Schluckspecht befindet sich also nicht mit einer Wahrscheinlichkeit von $75 \%$ in der letzten Kneipe. Damit dieser Fall eintreten würde, müssten die Voraussetzungen anders gewählt werden.

Zum Beispiel so: Herr von Schluckspecht entscheidet sich für einen der zwölf Orte. Statt dass Frau von Schluckspecht ihn nun suchen geht und damit bei jeder Kneipe aufs Neue die Chance hat, ihn zu finden, nennt ihr ein „Informant“ acht Stammkneipen, in denen er sich nicht befindet. Dann weiß Frau von Schluckspecht, dass ihr Mann sich mit einer Wahrscheinlichkeit von $75 \%$ in der letzten befindet.

L 10.2 Die einzigen solchen Quadratzahlen sind 1, 4 und 9 (und 0), d. h. genau die einstelligen Quadratzahlen.

Die Endziffer eines Produktes zweier Zahlen ist gerade die Endziffer des Produktes der Endziffern der beiden Zahlen: $(10 n+k)(10 m+t)=10(10 n m+t+k)+k t$.

Wegen $0 \cdot 0=\mathbf{0}, 1 \cdot 1=\mathbf{1}, 2 \cdot 2=\mathbf{4}, 3 \cdot 3=\mathbf{9}, 4 \cdot 4=1 \mathbf{6}, 5 \cdot 5=25,6 \cdot 6=3 \mathbf{6}$, $7 \cdot 7=49,8 \cdot 8=64$ und $9 \cdot 9=81$ enden alle Quadratzahlen auf $0,1,4,5,6$ oder 9 . 
Ist $n=2 k$ eine gerade Zahl, so ist ihr Quadrat $n^{2}=(2 k)^{2}=4 k^{2}$ durch 4 teilbar; ist $n=2 k+1$ eine ungerade Zahl, so ist $n^{2}=(2 k+1)^{2}=4\left(k^{2}+k\right)+1$, also ist $n^{2}-1$ durch 4 teilbar. Demnach ist eine Quadratzahl entweder selbst durch 4 teilbar oder ihr Vorgänger ist durch 4 teilbar.

Eine Zahl ist genau dann durch 4 teilbar, wenn die Zahl, die aus ihren beiden Endziffern gebildet wird, durch 4 teilbar ist. Da $11-1=10$ nicht durch 4 teilbar ist, ist auch jede Zahl 1..11-1 mit mindestens zwei Ziffern nicht durch 4 teilbar, deshalb ist $1 \ldots 11$ keine Quadratzahl. Genauso sind $55-1,55,66,66-1,99-1$ und 99 nicht durch 4 teilbar, also gibt es auch keine Quadratzahl mit mindestens zwei Stellen, die nur aus einer der Ziffern 5, 6 oder 9 besteht.

Bleiben also die einstelligen Quadratzahlen und Zahlen, die sich nur mit der Ziffer 4 schreiben lassen. Sei $n=k^{2}$ eine nur aus der Ziffer 4 bestehende Quadratzahl, dann ist $\frac{n}{4}=\frac{k^{2}}{2^{2}}=\left(\frac{k}{2}\right)^{2}$ eine Quadratzahl, die nur aus Einsen besteht. Deshalb ist $\frac{n}{4}=1$ (denn es gibt ja keine zwei- oder mehrstellige Quadratzahl nur aus der Ziffer 1, wie wir oben schon gezeigt haben) bzw. $n=4$.

Also sind alle Quadratzahlen, die sich mit lauter gleichen Ziffern schreiben lassen, einstellig.

Ach ja, der Vollständigkeit halber: Die einzige Zahl aus lauter Ziffern 0 ist natürlich die Null selbst.

L 10.3 Der Gesamtweg von Tom zu seinen Freundinnen ist am kürzesten, wenn Tom im gleichen Haus wie Emilia wohnt.

Wir beginnen links von A, nach rechts zu laufen, und schauen immer, wie sich die Summe der Weglängen ändert.

Wenn wir um eine Strecke $x$ nach rechts laufen und uns noch links von A aufhalten, werden die Weglängen zu jedem der Mädchen um $x$ kürzer, d.h. die Summe der Weglängen wird kürzer.

Wenn wir zwischen A und B um eine Strecke $x$ nach rechts laufen, werden die 8 Abstände zu den Mädchen B bis I jeweils um $x$ kürzer, während der Abstand zu A um $x$ länger wird. Zwischen $\mathrm{B}$ und $\mathrm{C}$ werden noch 7 Abstände um $x$ kürzer und schon 2 Abstände um $x$ länger. Aber immer noch wird die Abstandssumme kürzer, wenn wir nach rechts laufen. Genauso auf dem Weg von C nach D (6 Abstände um $x$ kürzer und 3 um $x$ länger) und von $\mathrm{D}$ nach $\mathrm{E}(5 \mathrm{zu} 4)$.

Wenn wir aber zwischen $\mathrm{E}$ und $\mathrm{F}$ um $x$ nach rechts laufen, werden 5 Abstände um $x$ länger und nur 4 Abstände um $x$ kürzer, d. h. die Abstandssumme wird um $x$ länger. Noch weiter zwischen $\mathrm{F}$ und $\mathrm{G}$ werden schon 6 Abstände um $x$ länger und nur noch 3 Abstände um $x$ kürzer, so dass die Summe der Abstände auch wieder größer wird, und so weiter, wenn wir noch weiter nach rechts gehen.

Die Summe der Abstände ist also gerade dann am kürzesten, wenn wir genau bei E sind. 
L 10.4 Wie gesagt, mit der Länge des Bandes kann man so direkt wenig anfangen. Proportional zur Länge des Bandes ist aber die Fläche der (ziemlich dünnen) Seite des Bandes. Also ist mit der Fläche des Bandes auf den Spulen zu rechnen: sie muss offensichtlich in der Summe immer gleich bleiben.

Sei wie in der Skizze beim Aufgabenblatt $a$ der Abstand des linken Spulenmittelpunkts vom linken Rand (und entsprechend auf der rechten Seite). Bei einem idealen Aufbau der Kassette hat eine volle Spule den Radius $a$, dann wird nach außen hin kein Platz verschenkt. Wird eine Kassette abgespielt, so nimmt, bis man bei der Hälfte angelangt ist, auf der rechten Spule der Radius schneller zu, als er links abnimmt, denn wegen des immer noch kleineren Radius auf der rechten Seite passt pro Umdrehung offensichtlich weniger Bandlänge auf die rechte Spule als auf die linke. Folglich ist die Summe der Radien genau in der Mittenposition des Bandes maximal, und für genau diesen Fall muss der Abstand der Spulen voneinander konzipiert sein: Im Idealfall ist dann kein messbarer Platz mehr zwischen den Spulen. Anders gesagt: die Spulen reichen dann genau bis zur Mitte der Kassette und haben also einen Radius von $50-a$.

Die Flächen des Bandes müssen in beiden Fällen gleich sein. Deren Berechnung erfolgt einfach über die Flächenformel des Kreises, wobei beachtet werden muss, dass die Fläche der Spulenkerne abgezogen wird. Es ergibt sich die Gleichung

$$
\begin{array}{rlrl} 
& \pi a^{2}-\pi 10,5^{2} & =2 \cdot\left(\pi(50-a)^{2}-\pi 10,5^{2}\right) \\
\Leftrightarrow & \pi a^{2}+\pi 10,5^{2} & = & 5000 \pi-200 \pi a+2 \pi a^{2} \\
\Leftrightarrow & 0 & = & a^{2}-200 a+4889,75 \\
\Leftrightarrow & a_{1,2} & =100 \pm \sqrt{10000-4889,75} \approx 100 \pm 71,486
\end{array}
$$

Da natürlich $a<100$ gelten muss, ist $a \approx 28,5 \mathrm{~mm}$ die optimale Lösung.

Im Rahmen der Messgenauigkeit ist das der reale Wert. In der Praxis muss man zwar noch Sicherheitsabstände einbeziehen, aber das muss nicht viel ändern: Gewährt man an der Seite und in der Mitte je $1 \mathrm{~mm}$ Reserve für die Spulen, erhält man $a \approx 28,6 \mathrm{~mm}$, was im Rahmen unserer Genauigkeit nichts ändert.

Für Videokassetten übrigens, die $188 \mathrm{~mm}$ breit sind und einen Spulenkern mit einem Radius von $13 \mathrm{~mm}$ haben, ergibt eine entsprechende Rechnung einen idealen Abstand von $a \approx 54,4 \mathrm{~mm}$. Real sind es aber nur etwa $48 \mathrm{~mm}$. Dieser große Unterschied ist wohl mit einer anderen Konstruktionsweise von Videokassetten zu erklären, deren Spulen noch Scheiben zur Bandführung haben. Da sich diese in der Mitte der Kassette nicht in die Quere kommen dürfen, kann $a$ bei maximalem Spulenradius nur ein Viertel der Kassettenbreite betragen. Und das ist ziemlich genau der Fall. 


\section{Lösungen zu Aufgabenblatt 11}

L 11.1 Zur Lösung der Aufgabe konzentrieren wir uns zunächst ganz auf die Buchstaben $N, A, B$ und $E$. Aus den Einerstellen der ersten Spalte (NENA : $E N=R A N)$ ergibt sich, dass $A$ die Einerstelle von $N \cdot N$ ist. Ebenso ergibt sich aus der dritten Zeile, dass $B$ die Einerstelle von $N \cdot A$ ist. Mit der Wahl von $N$ sind also $B$ und $A$ festgelegt, dafür fertigen wir uns eine Tabelle an:

\begin{tabular}{c|c|c|c|c|c|c|c|c|c|c}
$N$ & 0 & 1 & 2 & 3 & 4 & 5 & 6 & 7 & 8 & 9 \\
\hline$A$ & 0 & 1 & 4 & 9 & 6 & 5 & 6 & 9 & 4 & 1 \\
\hline$B$ & 0 & 1 & 8 & 7 & 4 & 5 & 6 & 3 & 2 & 9 \\
\hline \hline & - & - & + & + & - & - & - & + & + & -
\end{tabular}

Verschiedene Buchstaben stehen für verschiedene Ziffern, deshalb scheiden alle mit "- " gekennzeichneten Spalten aus.

Als Nächstes betrachten wir die zweite Zeile. Dort wird die zweistellige Zahl EN zu $B A S E$ hinzuaddiert, dabei kann sich die Hunderterstelle höchstens um eins erhöhen. Also ist $E=A+1$. Wir können somit unsere Tabelle noch um eine Zeile erweitern, wobei wir jetzt nur die oben noch nicht ausgeschlossenen Spalten berücksichtigen:

\begin{tabular}{l|l|l|l|l}
$N$ & 2 & 3 & 7 & 8 \\
\hline$A$ & 4 & 9 & 9 & 4 \\
\hline$B$ & 8 & 7 & 3 & 2 \\
\hline$E$ & 5 & 0 & 0 & 5 \\
\hline \hline & + & - & - & +
\end{tabular}

$E$ darf nicht 0 sein, z. B. wegen der Einerstelle in der ersten Zeile, deshalb sind die beiden mit Minus gekennzeichneten Spalten nicht möglich. Somit ist $E=5$ und $A=4$.

Schließlich betrachten wir noch die Tausenderstellen in der ersten Zeile und der letzten Spalte des Kryptogramms. Wegen der letzten Spalte ist $O$ größer als $B$ und $T$ und damit mindestens 2. Aus dem gleichen Grund ist wegen der ersten Zeile $N$ größer als $O$, also ist $N \geq 3$. Damit scheidet auch die erste Spalte der Tabelle aus. Übrig bleiben damit $N=8$ und $B=2$.

Den Rest kann man leicht vervollständigen, indem man die schon bestimmten Buchstaben durch die entsprechenden Zahlen ersetzt und die sich dadurch ergebenden 
Operationen ausführt.

Es ergibt sich als einzige Lösung:

$$
\begin{array}{ccccc}
8584 & - & 2499 & = & 6085 \\
: & & - & & - \\
58 & + & 2475 & = & 2533 \\
= & & = & & = \\
148 & * & 24 & & \\
14852
\end{array}
$$

Die abschließende Probe zeigt, dass auch wirklich alle sechs Gleichungen des Kryptogramms erfüllt sind. (Die Probe ist wichtig, denn wenn z. B. aus einer Gleichung $N=8$ folgt, heißt das ja noch nicht, dass dies nicht in einer anderen Gleichung zu einem Widerspruch führt, deshalb muss man am Ende überprüfen, ob auch wirklich alle Gleichungen wahre Aussagen sind.)

L 11.2 Der Zielpunkt $B$ liegt nordöstlich von $A$, und zwar $3 \cdot 60 \mathrm{~m}=180 \mathrm{~m}(3$ Rastereinheiten) nördlich und $7 \cdot 60 \mathrm{~m}=420 \mathrm{~m}$ (7 Rastereinheiten) östlich von $A$. In der Summe von Ost- und Nord-Richtung muss Rudi also $600 \mathrm{~m}$ zurücklegen. Es ist an sich klar, dass es für Rudi nur Sinn macht, sich im Raster der Stadt in nördlicher oder in östlicher Richtung zu bewegen. (Am Ende wird das noch einmal kritisch untersucht.)

Grundsätzlich gibt es für einen beliebigen Abschnitt von Rudis Fahrtstrecke zwei Möglichkeiten:

- Entweder befindet er sich auf einem geraden Abschnitt in Nord- oder OstRichtung. Dann legt er pro gefahrenen Meter auch genau einen Meter in der Summe von Ost- und Nord-Richtung zurück.

- Oder er befährt eine der Abbiegekurven, die ihn zwischen diesen Richtungen wechseln lässt. Diese haben eine Länge von $\frac{1}{4}(2 \pi \cdot 20 \mathrm{~m}) \approx 31,416 \mathrm{~m}$, und er legt dabei je $20 \mathrm{~m}$ in Nord- und in Ost-Richtung zurück, in der Summe der beiden Richtungen also $40 \mathrm{~m}$ und demnach mehr, als er gefahren ist.

Er sollte daher so viele Kurven wie möglich befahren.

Nun liegt an jeder Kurve, die Rudi sinnvoll befahren kann, im Raster der Stadt ein gerader Abschnitt in nördlicher Richtung und einer in östlicher Richtung. Da er drei Rasterabschnitte nach Norden fahren muss, kann er also maximal sechs Kurven befahren, denn an jeden Abschnitt stoßen ja höchstens zwei Kurven.

Der Stadtsee bildet im Verkehrsnetz ein natürliches Hindernis. Fährt Rudi südlich daran vorbei, so muss er vorher zwei Kurven befahren haben (wofür es zwei Möglichkeiten gibt) und hat danach, wie man leicht durch Probieren feststellen kann, drei 


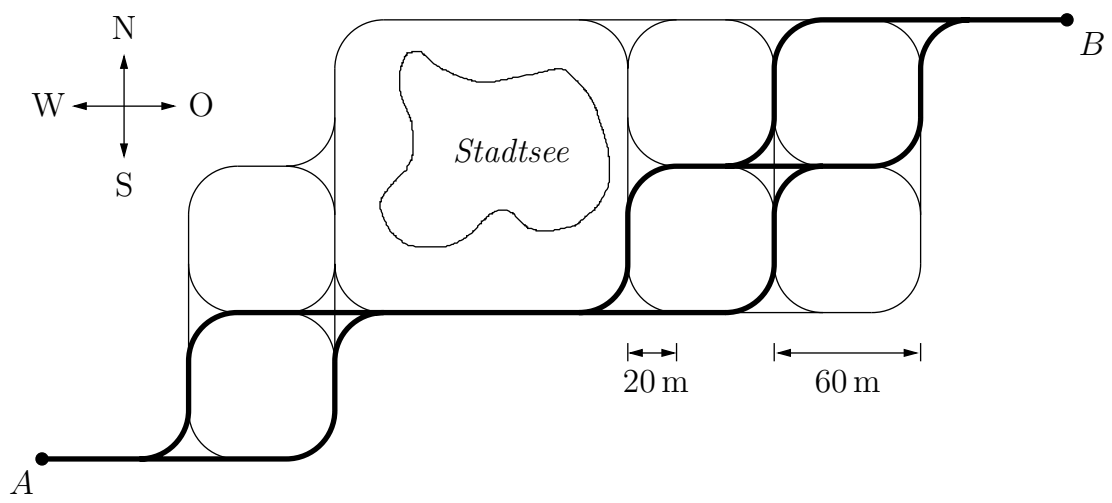

Abbildung 11.1: Rudis kürzeste Tour durch Neu-Weende

Möglichkeiten, um mit vier weiteren Kurven zum Ziel zu kommen. Die verschiedenen Verzweigungen sind in der Skizze oben verzeichnet, deren Darstellungsweise der Lösung von Julia entnommen wurde. Auf der südlichen Route hat Rudi also $2 \cdot 3=6$ Varianten.

Wenn Rudi nördlich am See vorbeifahren will, kann er danach nur noch geradeaus fahren. Davor kann er aber maximal vier Kurven genutzt haben, also ist dieser Weg grundsätzlich länger.

Er hat also 6 Möglichkeiten für einen kürzestmöglichen Weg, und diese haben eine Länge von ca. $600 \mathrm{~m}-6 \cdot 40 \mathrm{~m}+6 \cdot 31,416 \mathrm{~m} \approx 548,5 \mathrm{~m}$.

Um deutlich zu machen, dass es sich lohnt, sich noch einmal Gedanken über die grundsätzlichen Fahrtmöglichkeiten von Rudi zu machen, sei ein anderes Straßennetz gezeigt (siehe Abbildung 11.2):

Hier existiert zwar ein Weg, der der kürzeste wäre, wenn die Ecken im Straßengitter nicht abgerundet wären (über $C$, Länge: 1062,8 m). Aber wegen der vielen Kurven ist der Weg über $D(1045,5 \mathrm{~m})$ tatsächlich kürzer, auch wenn er im nicht-abgerundeten Fall offensichtlich länger als der andere wäre.

Man muss also Folgendes betrachten:

- Wenn Rudi eine der Kurven, die für ihn eigentlich günstig wären, in der Gegenrichtung befährt, verliert er $40 \mathrm{~m}$ in der Entfernungssumme, obwohl sich seine Fahrtstrecke um weitere $31,416 \mathrm{~m}$ verlängert.

- Auf den übrigen Kurven gewinnt er in der einen Richtung zwar 20 m, verliert diese aber dafür in der anderen Richtung.

- Fährt er auf einem geraden Abschnitt in südlicher oder westlicher Richtung, verliert er pro gefahrenem Meter in der Summe von Ost- oder Westrichtung genau einen Meter. 


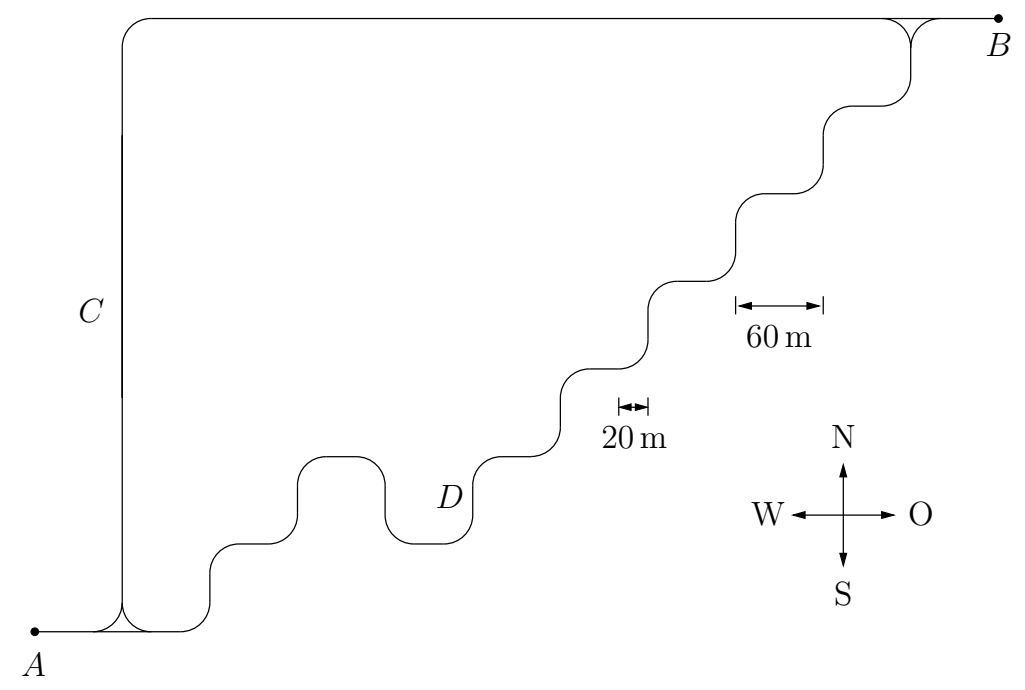

Abbildung 11.2: Straßennetz, in dem ein „Umweg“ eine Abkürzung ist

Alle diese Möglichkeiten sind also ungünstiger als die oben angeführten. Aber es kann unter Umständen sein (wie oben), dass solche „schlechten“ Abschnitte durch viele Nord-Ost-Kurven wieder ausgeglichen werden.

In Neu Weende ist nun die Situation auf die Art „angenehm“, dass es Wege gibt, die sowohl auf dem Quadratgitter minimal kurz wären als auch die (auf dem vollständigen Gitter) maximal mögliche Zahl von Abbiegevorgängen enthalten (hier also sechs, dadurch gegeben, dass Rudi nur drei Rasterabschnitte nach Norden fahren muss). Wenn er hier mehr Kurven in nordöstlicher Richtung fahren wollte, so müsste er nach den obigen Überlegungen also noch einen Abschnitt mehr in nördlicher Richtung fahren. Dafür muss er dann aber irgendwann einmal zum Ausgleich einen Abschnitt nach Süden fahren und braucht dafür natürlich auch noch zwei Kurven, um dorthin ein- und wieder auszuschwenken. Das ergibt also zumindest vier Kurven, die zu fahren sind, um noch maximal zwei Kurven mit dem besten Entfernungsgewinn-zuStreckenlängen-Verhältnis zu benutzen. Diese bedeuten einen Fahrweg von 125,7 m, Rudi gewinnt dabei aber maximal $80 \mathrm{~m}$ Entfernung (in östlicher Richtung). Also macht es in Neu Weende keinen Sinn, mehr als sechs Kurven zu benutzen.

L 11.3 a) Yvonne kann den Gewinn erzwingen.

Wir betrachten zuerst Spielvarianten mit weniger Hölzchen. Bei einem Hölzchen verliert natürlich der Spieler, der am Zug ist (d.h. der anziehende Spieler), weil er das Hölzchen wegnehmen muss. Bei zwei bis acht Hölzchen gewinnt der anziehende Spieler, da er gerade so viel wegnehmen kann, dass noch ein Hölzchen übrig bleibt, das 
Lösungen zu Aufgabenblatt 11

dann der andere wegnehmen muss. Bei neun Hölzchen verliert dagegen der Anziehende wieder. Er kann ja nur ein bis sieben Hölzchen wegnehmen und dann entsteht die Situation mit zwei bis acht Hölzchen, die wir gerade hatten, nur dass diesmal der andere in dieser Situation beginnt. Man sieht hier schon, dass man die Situation rekursiv bewerten kann, und zwar folgendermaßen: Bei $n$ Hölzchen verliert der Anziehende (im Folgenden Verluststellung genannt), wenn er nur Situationen herstellen kann, in denen der Anziehende (der ja dann der andere ist) gewinnt. Andererseits gewinnt der Anziehende (so genannte Gewinnstellung), wenn man die Möglichkeit hat, in eine Verluststellung zu ziehen. Die Tabelle 11.1 zeigt die Bewertungen für ein bis 26 Hölzchen. ( $\mathrm{G}$ bedeutet Gewinnstellung, V bedeutet Verluststellung.)

\begin{tabular}{c|ccccccccccccc} 
Hölzchen & 1 & 2 & 3 & 4 & 5 & 6 & 7 & 8 & 9 & 10 & 11 & 12 & 13 \\
\hline Stellung & V & G & G & G & G & G & G & G & V & G & G & G & G \\
Hölzchen & 14 & 15 & 16 & 17 & 18 & 19 & 20 & 21 & 22 & 23 & 24 & 25 & 26 \\
\hline Stellung & G & G & G & V & G & G & G & G & G & G & G & V & G
\end{tabular}

Tabelle 11.1: Bewertung der Stellungen im Aufgabenteil a)

Wir haben also immer eine Verluststellung und dann sieben Gewinnstellungen, dann wieder eine Verluststellung und so weiter. Also haben wir genau dann eine Verluststellung, wenn die Anzahl der Hölzchen geteilt durch 8 den Rest 1 lässt.

Daraus können wir dann folgern, dass bei 100 Hölzchen (die in der Aufgabe vorgegeben sind) Yvonne gewinnt, da $100: 8=12$ Rest 4, und zwar nimmt sie am Anfang 3 Hölzchen und dann immer 8 minus die Anzahl der Hölzchen, die Zacharias genommen hat.

b) Hier kann Zacharias den Gewinn erzwingen.

Wir finden eine Gewinnstrategie ähnlich wie in Teil a). Es wird nur ein bisschen schwieriger, weil wir jetzt zwei Haufen haben. Wenn ein Haufen kein Hölzchen enthält, haben wir die Situationen von Teil a), bei denen wir schon wissen, ob es Gewinn- oder Verluststellungen sind. Nach den gleichen Überlegungen wie in Teil a) (Verluststellung, wenn man nur in Gewinnstellungen ziehen kann, und Gewinnstellung, wenn man die Möglichkeit hat, in eine Verluststellung zu ziehen) erhalten wir Tabelle 11.2, wobei die Zahlen in der ersten Zeile für die Anzahl der Hölzchen des einen Haufens und die Zahlen in der ersten Spalte für die Anzahl der Hölzchen im anderen Haufen stehen.

Man muss hier natürlich beachten, dass man bis zu 7 Hölzchen vom einen oder vom anderen Haufen ziehen kann, d. h. wir setzen ein V an eine Stelle, wenn sowohl an den sieben Stellen darüber als auch an den sieben Stellen links davon nur G stehen, und 


\begin{tabular}{c|cccccccc|ccccc} 
& 0 & 1 & 2 & 3 & 4 & 5 & 6 & 7 & 8 & 9 & 10 & 11 & 12 \\
\hline 0 & & V & G & G & G & G & G & G & G & V & G & G & G \\
1 & V & G & G & G & G & G & G & G & V & G & G & G & G \\
2 & G & G & V & G & G & G & G & G & G & G & V & G & G \\
3 & G & G & G & V & G & G & G & G & G & G & G & V & G \\
4 & G & G & G & G & V & G & G & G & G & G & G & G & V \\
5 & G & G & G & G & G & V & G & G & G & G & G & G & G \\
6 & G & G & G & G & G & G & V & G & G & G & G & G & G \\
7 & G & G & G & G & G & G & G & V & G & G & G & G & G \\
\hline 8 & G & V & G & G & G & G & G & G & G & V & G & G & G \\
9 & V & G & G & G & G & G & G & G & V & G & G & G & G \\
10 & G & G & V & G & G & G & G & G & G & G & V & G & G
\end{tabular}

Tabelle 11.2: Bewertung der Stellungen im Aufgabenteil b)

falls an einer dieser Stellen ein V steht, setzen wir ein G. Wieder kann man erkennen, dass sich die Verlust- und Gewinnstellungen in Achterschritten wiederholen, und zwar sowohl nach rechts als auch nach unten.

Als Ergebnis erhält man, dass man sich in einer Verluststellung befindet, wenn die Reste der Hölzchenanzahl der Haufen beim Teilen durch 8 gleich sind und eine der Zahlen $2,3, \ldots, 7$ oder wenn der eine Rest gleich 0 und der andere gleich 1 ist.

In der Aufgabe haben beide Haufen die Größe $100=8 \cdot 12+4$, d. h. beide Anzahlen geben beim Teilen durch 8 den Rest 4 . Wir haben somit eine Verluststellung. Da Yvonne anfängt, kann Zacharias den Gewinn erzwingen. Hier gibt es mehrere Spielstrategien für Zacharias. Eine wäre nach unserer Überlegung folgende: Solange Yvonne von einem Haufen zieht, der mehr als 8 Hölzchen enthält, zieht Zacharias vom gleichen Haufen gerade 8 minus die Anzahl der von Yvonne gezogenen Hölzchen. Auf diese Weise tritt irgendwann die Situation ein, dass ein Haufen 4 Hölzchen und der andere $4+8 k$ Hölzchen enthält (wir hatten ja zu Anfang je $100=4+8 \cdot 12$ Hölzchen).

Zieht Yvonne von dem 4er-Haufen nun 1 oder 2 Hölzchen, so zieht Zacharias 1 bzw. 2 Hölzchen von dem anderen Haufen und sie gelangen in eine Situation wie $(3,11)$ bzw. $(2,10)$. Zieht Yvonne von dem 4er-Haufen aber 3 oder 4 Hölzchen, so zieht Zacharias von dem anderen Haufen 4 bzw. 3 Hölzchen, so dass sie in eine Situation wie $(1,8)$ bzw. $(0,9)$ gelangen. Zieht Yvonne von dem größeren Haufen (mit mehr 
Lösungen zu Aufgabenblatt 11

als acht Hölzchen), so zieht Zacharias auch wieder vom gleichen Haufen den Rest zu 8 Hölzchen. Usw.

Bemerkung: Es ist nicht der richtige Weg, seine Gewinnstrategie auf Grund von Annahmen über die Spielstrategie seines Gegners zu machen. Die Gewinnstrategie muss so sein, dass der Gegner bei jeglicher Spielweise verliert.

L 11.4 Es gibt viele Möglichkeiten, wie der Raum aussehen könnte und an welcher Stelle Paul Sorglos sitzt. Eine Möglichkeit ist in der Abbildung 11.3 dargestellt.

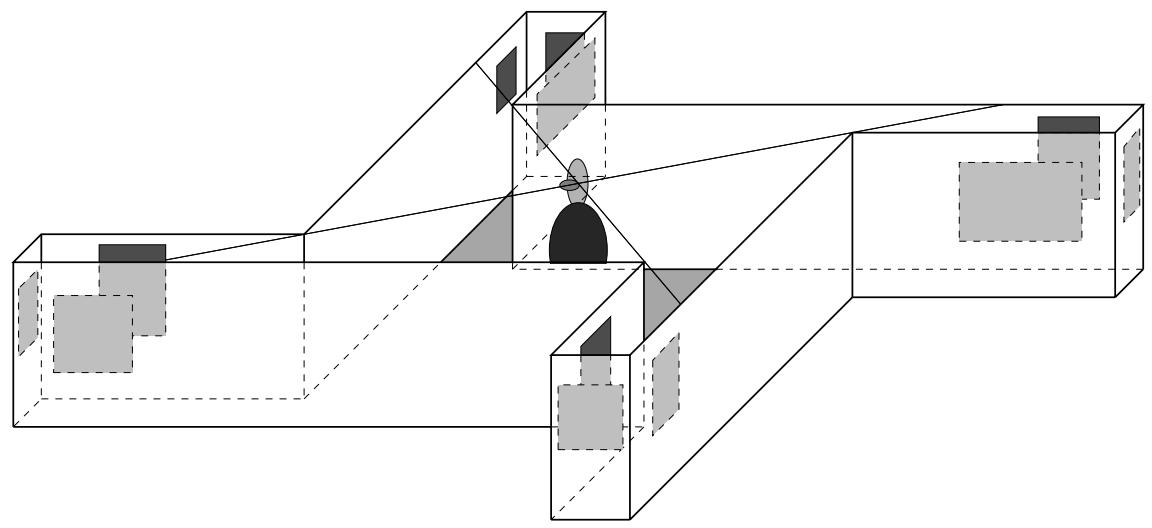

Abbildung 11.3: Ein möglicher Raum

An jeder Wand dieses Raumes ist die Position eines Bildes angedeutet, die Paul von seinem Sitzplatz im Zentrum des Raumes nicht einsehen kann.

Damit ist die Aufgabe gelöst. Es gibt aber eine Reihe interessanter Fragestellungen, die sich anschließen könnten:

- Man sieht schnell, dass die abgebildete Variante mit zwölf Wänden (also zwölf entwendeten Bildern) nicht der Raum mit der kleinsten Anzahl an Wänden ist, den man sich denken kann - zum Beispiel kann man in obigem Bild in den vier „kleinen Seitenräumen“ die „kleinen“ Außenwände immer kleiner machen, bis die Seitenräume nur noch Spitzen sind. Der resultierende Raum hat dann nur noch acht Wände. Geht man von einer gleichseitig dreieckigen Grundform des Raumes aus, so kann man eine ähnliche Konstruktion machen und der entstehende Raum hat nur noch sechs Wände.

Kann man einen solchen Raum mit weniger als sechs Wänden finden? 
- Kann man den Raum so bauen, dass zusätzlich folgende Aussage gilt:

Es gibt innerhalb des Raumes einen Weg, auf dem die Diebe ihre Beute einsammeln konnten, ohne dass Paul Sorglos sie bemerken konnte.

Wie viele Wände muss ein solcher Raum dann mindestens haben?

- In einer fernen Zukunft „schweben“ die Besucher durch die beliebig verwinkelten dreidimensionalen Räume, das heißt, es gibt in diesen Museen keine Decke und keinen Boden mehr - an jeder Innenfläche der räumlichen Gebilde hängen Bilder.

Ist es möglich, dass ein Enkel von Paul Sorglos dasselbe Unheil wie sein Opa erlebt? Oder: Gibt es einen Polyeder (also einen Vielflächner im Raum), in dessen Innerem es einen Punkt gibt, von dem aus man keine der Seitenflächen vollständig sieht? Und wie viele Flächen muss solch ein Polyeder dann mindestens haben? 


\section{Lösungen zu Aufgabenblatt 12}

L 12.1 Wir bezeichnen den Spieler, der die erste Runde verloren hat, mit $A$, den Verlierer der zweiten Runde mit $B$ und den der dritten Runde mit $C$. Diese Zuordnung ist eindeutig, da jeder Spieler genau eine Runde verloren hat. Nach der dritten Runde hat jeder Spieler genau 24 Punkte:

\begin{tabular}{l|l|l|l} 
Spieler & $A$ & $B$ & $C$ \\
\hline Punkte nach der dritten Runde & 24 & 24 & 24
\end{tabular}

Spieler $C$ hat die dritte Runde verloren, also wurden die Punktzahlen von $A$ und $B$ verdoppelt. Folglich hatten diese beiden Spieler vor der dritten Runde jeweils 12 Punkte, jeder hat 12 Punkte dazubekommen. Diese 24 Punkte wurden $C$ abgezogen, also hatte $C$ vor der dritten Runde 48 Punkte:

\begin{tabular}{l|c|c|c} 
Spieler & $A$ & $B$ & $C$ \\
\hline Punkte nach der zweiten Runde & 12 & 12 & 48 \\
Punkte nach der dritten Runde & 24 & 24 & 24
\end{tabular}

In der zweiten Runde hatte $B$ verloren, also wurden die Punktzahlen von $A$ und $C$ verdoppelt. Spieler $A$ hatte also vorher 6 Punkte und Spieler $C$ hatte 24 Punkte. Die $6+24=30$ Punkte wurden Spieler $B$ in der zweiten Runde abgezogen, also hatte er vorher $12+30=42$ Punkte:

\begin{tabular}{l|c|c|c} 
Spieler & $A$ & $B$ & $C$ \\
\hline Punkte nach der ersten Runde & 6 & 42 & 24 \\
Punkte nach der zweiten Runde & 12 & 12 & 48 \\
Punkte nach der dritten Runde & 24 & 24 & 24
\end{tabular}

Spieler $A$ hat die erste Runde verloren, folglich hatte $B$ vor der ersten Runde 21 und $C$ hatte 12 Punkte. Spieler $A$ wurden $12+21=33$ Punkte abgezogen, er hatte also vorher $6+33=39$ Punkte:

\begin{tabular}{l|c|c|c} 
Spieler & $A$ & $B$ & $C$ \\
\hline Punkte zu Beginn: & 39 & 21 & 12 \\
Punkte nach der ersten Runde & 6 & 42 & 24 \\
Punkte nach der zweiten Runde & 12 & 12 & 48 \\
Punkte nach der dritten Runde & 24 & 24 & 24
\end{tabular}


Antwort: Der Spieler, der die erste Runde verlor, hatte zu Beginn 39 Punkte, der Verlierer der zweiten Runde hatte zu Beginn 21 Punkte und der Verlierer der dritten Runde hatte 12 Punkte. Bei dieser Ausgangskonstellation und diesem Spielverlauf haben nach drei Runden alle 24 Punkte.

L 12.2 Es ist völlig egal, wo Peter seinen Liegestuhl aufstellt.

Betrachten wir eine Skizze des Gartens in Abbildung 12.1. Die Eckpunkte seien A, $B$ und $C$ und die Länge der Seiten sei $a$.

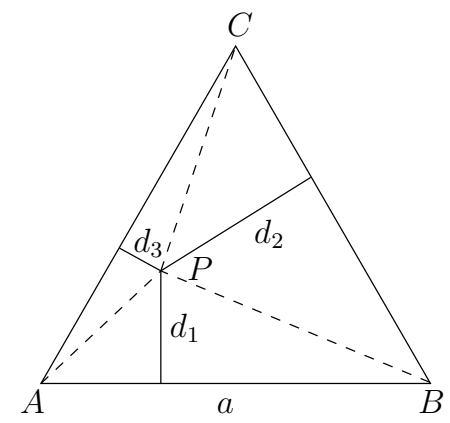

Abbildung 12.1: Skizze von Peters Garten

$P$ bezeichne den Platz, an dem Peter seinen Liegestuhl aufstellen könnte. Wir berechnen nun die Strecke, die Peter zurücklegen müsste.

Offensichtlich ist Peters Weg zu einem der Zäune am kürzesten, wenn er direkt senkrecht darauf zu läuft. Bezeichnen wir also die Abstände von $P$ zu den Seiten mit $d_{1}, d_{2}$ und $d_{3}$ (vgl. Zeichnung).

Peters Gesamtweg ist dann $2 d_{1}+2 d_{2}+2 d_{3}$.

Der große Trick:

Wir zerlegen das Dreieck $A B C$ in die kleinen Dreiecke $A B P, B C P$ und $C A P$. Die Flächeninhalte dieser kleinen Dreiecke sind dann $F_{1}=\frac{1}{2} d_{1} a, F_{2}=\frac{1}{2} d_{2} a$ bzw. $F_{3}=\frac{1}{2} d_{3} a$. Zusammen ergeben sie aber gerade das Dreieck $A B C$, d. h.

$$
F_{1}+F_{2}+F_{3}=\frac{1}{2} h a,
$$

wobei $h$ die Höhe des Dreiecks $A B C$ bezeichne. Und daraus folgt:

$$
\frac{1}{2} d_{1} a+\frac{1}{2} d_{2} a+\frac{1}{2} d_{3} a=\frac{1}{2} h a,
$$

also

$$
d_{1}+d_{2}+d_{3}=h .
$$

Somit beträgt der Weg, den Peter zurücklegen muss, immer $2 h$. 
Lösungen zu Aufgabenblatt 12

L 12.3 Aus Perlen in drei Farben kann man genau 39 verschiedene Armbänder à 5 Perlen fertigen.

Einfarbige Armbänder

$(5,0,0)^{1}$

Da es Perlen in drei Farben gibt, kann man genau drei unterschiedliche jeweils einfarbige Armbänder herstellen.

Armbänder in zwei Farben

$(4,1,0)$

Bei vier gleichfarbigen Perlen und einer andersfarbigen Perle ist es egal, an welcher Stelle im „Kreis“ die andersfarbige Perle ist. Für die erste Farbe hat man drei Möglichkeiten, und für jede dieser Wahlen kann man sich aus den zwei verbleibenden Farben noch die andersfarbige Perle aussuchen.

6

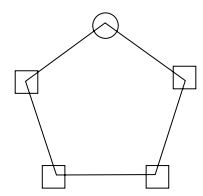

$(3,2,0)$

Nimmt man drei Perlen einer ersten und zwei Perlen einer zweiten Farbe, so kann man sich aussuchen, ob man die beiden Perlen der zweiten Farbe nebeneinander aufreiht oder ob man sie trennt (im zweiten Fall befinden sich zwischen ihnen auf der einen Seite zwei Perlen und auf der anderen Seite eine Perle der ersten Farbe). Auch hier gibt es wieder 6 Möglichkeiten, die Farbkombination zu wählen. $\mathbf{2} \cdot \mathbf{6}=\mathbf{1 2}$

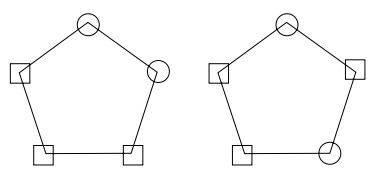

Armbänder in allen drei Farben

$(3,1,1)$

Nimmt man drei Perlen einer Farbe und jeweils eine Perle der anderen beiden Farben, so hat man genau wie im letzten Fall die beiden Möglichkeiten, die beiden einzelnen Perlen zu trennen oder sie zusammen zu lassen. Hier kann man sich aber nur die Farbe der drei gleichfarbigen Perlen aussuchen.

$2 \cdot 3=6$

${ }^{1}$ Dieses Tripel gibt jeweils den Typ der Farbverteilung an, hier: 5 Kugeln von einer ersten Farbe, 0 von einer zweiten und 0 von der letzten. 


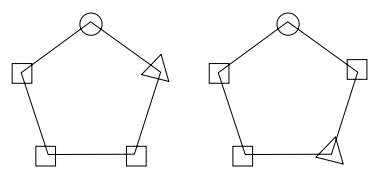

$(2,2,1)$

Nimmt man zwei Perlen einer ersten, zwei Perlen einer zweiten und eine Perle einer dritten Farbe, so gibt es wieder nur drei Möglichkeiten für die Farbwahl, nämlich bei der Farbe der einzelnen Perle.

Hier hat man jeweils die vier Möglichkeiten, beide Paare gleichfarbiger Perlen, keines der beiden, nur das erste oder nur das zweite zu trennen.

$3 \cdot 4=12$
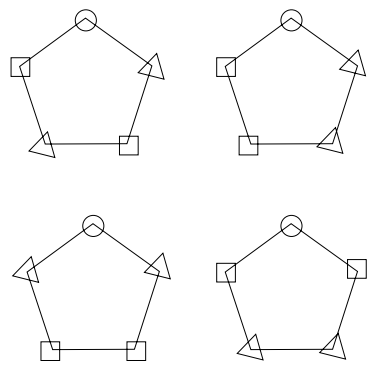

Das Ergebnis

Addieren liefert $3+6+12+6+12=39$ Möglichkeiten.

L 12.4 Wie die Aufgabenstellung schon vermuten lässt, gibt es hier nicht die richtige Lösung, sondern viele Möglichkeiten. Einige davon sind die folgenden:

1. Eine erste Idee könnte sein, die maximale Nord-Süd- und Ost-West-Ausdehnung zu bestimmen und dann diese Abstände jeweils zu halbieren. Das ist eine recht „grobe“ Methode in dem Sinne, dass sie nur wenige Informationen der Karte nutzt. Jede Karte mit denselben Ausdehnungen liefert denselben Mittelpunkt. Deutschland ohne Bayern würde zum Beispiel dasselbe Ergebnis liefern.

Aber im Ergebnis erhält man einen dem Auge als Mittelpunkt gefälligen, wenn auch anscheinend etwas zu weit östlich gelegenen Punkt mit den Koordinaten (8;10) (wenn der Ursprung des Koordinatensystems links unten liegt), siehe Abbildung 12.2.

Auf einer echten Deutschlandkarte liegt dies in der Nähe von Meiningen in Thüringen. 


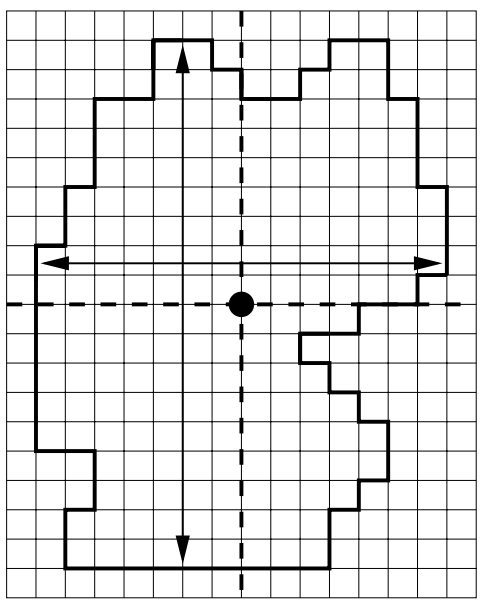

Abbildung 12.2: Erste Methode

2. Als Abwandlung dieser Methode kann man nun noch berücksichtigen, dass bei zwei Karten mit der gleichen Nord-Süd-Ausdehnung, jedoch verschiedener Verteilungen der Flächen in dieser Richtung (zum Beispiel könnte die eine nach unten hin, die andere nach oben hin schmaler werden) auch die Mittelpunkte verschieden sein sollten. Vom Mittelpunkt aus gesehen sollte in beiden Richtungen gleich viel Fläche sein (nicht Abstand, wie im ersten Versuch). Man kann also die Gesamtzahl der Kästchen der Karte zählen - das sind 186 Stück - und dann, ebenfalls durch Zählen, genau die senkrechte und waagerechte Linie bestimmen, die die Fläche halbiert (siehe Abbildung 12.3).

Im Ergebnis der ersten Methode schien der Punkt zu weit im Osten zu liegen. Ursache hierfür war, dass diese Methode die geringere Landmasse im Osten nicht beachtete. Dies wurde hier augenscheinlich besser berücksichtigt. Der erhaltene Punkt $(7,41 ; 10,23)$ liegt bei Kaltennordheim in der Nähe der thüringisch-hessischen Grenze.

3. Man kann auch ein wenig physikalisch an die Fragestellung herangehen und versuchen, den Schwerpunkt der Landmassenverteilung zu bestimmen. Der Schwerpunkt ist dabei bildlich der Punkt, an dem man die Karte, als Pappscheibe gedacht, so aufhängen kann, dass diese waagerecht hängt. Diesen zu bestimmen hat man wiederum mehrere Möglichkeiten:

a) Man tut genau das, was eben beschrieben wurde: Aus Pappe ausschneiden und versuchen, an einem Punkt waagerecht aufzuhängen. 


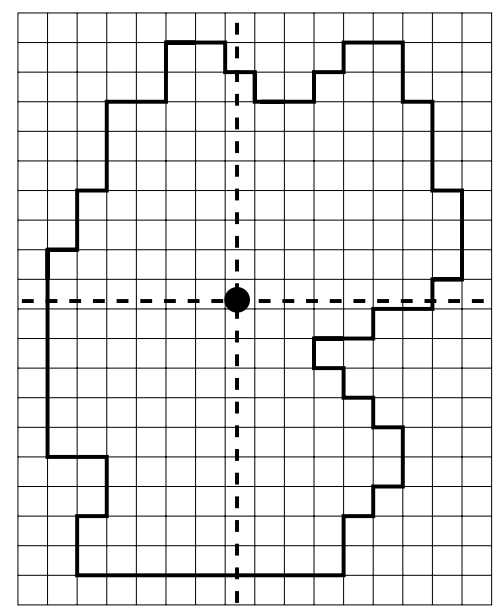

Abbildung 12.3: Zweite Methode

b) Man schneidet die Karte aus Pappe aus und hängt die Scheibe an irgendeinem (Rand-)Punkt auf. Dann liegt der Schwerpunkt irgendwo direkt unterhalb des Aufhängepunktes. Zeichnet man also auf der Karte diese Gerade nach unten und wiederholt die Prozedur mit einem zweiten Aufhängepunkt, so schneiden sich die entstehenden Geraden im Schwerpunkt.

c) Man rechnet: Hierbei denkt man sich formal an jedem kleinen Quadrat eine Masse $m$ (die kleinen Quadrate haben ja gleich große Flächen, daher die gleiche Masse). Im Unterschied zur Methode Nummer 2 gehen bei der Berechnung des Schwerpunktes jetzt nicht nur die Größe der links und rechts gelegenen Fläche, sondern auch deren Abstand vom Schwerpunkt ein - weit weg gelegene Flächenstücke zählen mehr. Sind $\left(x_{i} ; y_{i}\right)$ mit $1 \leq i \leq N$ die Positionen der Massen (Mittelpunkte der Quadrate), so ist der Schwerpunkt gerade der Mittelwert dieser Massenpunkte:

$$
\begin{aligned}
S & =\left(\frac{1}{N \cdot m}\left(m \cdot x_{1}+\ldots+m \cdot x_{N}\right), \frac{1}{N \cdot m}\left(m \cdot y_{1}+\ldots+m \cdot y_{N}\right)\right) \\
& =\left(\frac{1}{N}\left(x_{1}+x_{2}+\ldots+x_{N}\right), \frac{1}{N}\left(y_{1}+y_{2}+\ldots+y_{N}\right)\right) .
\end{aligned}
$$

Für eine allgemeine Verteilung von $N$ Massenpunkten $\left(x_{i} ; y_{i}\right)$ verschiedener Massen $m_{i}$ mit der Gesamtmasse $M=m_{1}+m_{2}+\ldots+m_{N}$ ist der 
Schwerpunkt übrigens:

$$
S=\left(\frac{1}{M}\left(m_{1} \cdot x_{1}+\ldots+m_{N} \cdot x_{N}\right), \frac{1}{M}\left(m_{1} \cdot y_{1}+\ldots+m_{N} \cdot y_{N}\right)\right) .
$$

All diese Methoden sollten einen etwa bei $(7,53 ; 9,84)$ gelegenen Schwerpunkt ergeben (siehe Abbildung 12.4).

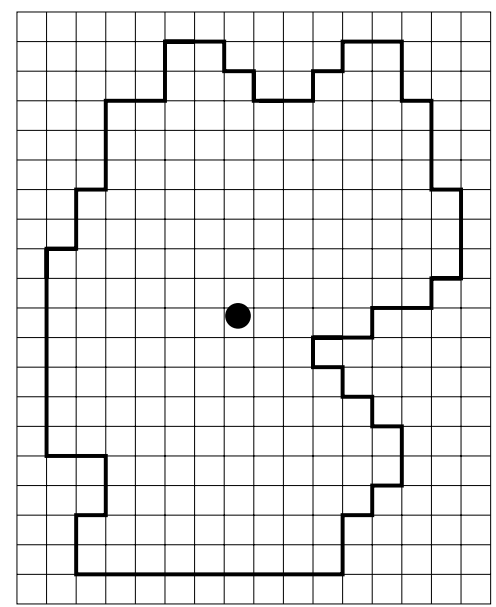

Abbildung 12.4: Dritte Methode

Der Punkt liegt etwa an der gleichen Ost-West-Koordinate, aber etwas südlicher als der bei der zweiten Methode bestimmte Punkt. Der Grund hierfür wurde schon verraten: Die waagerechte Flächenhalbierungsgerade liegt näher am oberen Rand der Karte als am südlichen - für den Schwerpunkt fallen aber weit weg gelegene Teile mehr ins Gewicht.

Mehr zum Thema Mittelpunkt Deutschlands findet man im Internet zum Beispiel unter http://www.eichsfeld-online.de/mittelpunkt/. Dort werden mit ähnlichen Methoden wie hier beschrieben insgesamt fünf verschiedene Orte bestimmt, die alle von sich behaupten, Mittelpunkt Deutschlands zu sein. 


\section{Lösungen zu Aufgabenblatt 13}

L 13.1 Die Drillinge können den Gesamtbetrag niemals unter sich aufteilen. An den ersten Tagen ist die geschenkte Summe nicht durch 3 teilbar, wie folgende Tabelle zeigt:

\begin{tabular}{c|c|c} 
Tag $t$ & Summe $S(t)$ & Rest bei Division durch 3 \\
\hline 0 & 100 & 1 \\
1 & 101 & 2 \\
2 & 103 & 1 \\
\hline 3 & 106 & 1 \\
4 & 110 & 2 \\
5 & 115 & 1 \\
\hline 6 & 121 & 1 \\
7 & 128 & 2 \\
8 & 136 & 1
\end{tabular}

Betrachtet man die Tabelle genauer, so liegt die Vermutung nahe, dass sich die Reste in Dreiergruppen wiederholen.

Hierzu berechnen wir, um wie viel sich die Summe am $(n+3)$-ten Tag von der Summe am $n$-ten Tag unterscheidet:

$$
\begin{aligned}
S(n+3) & =100+1+2+\cdots+(n+3) \\
& =100+1+2+\cdots+n+((n+1)+(n+2)+(n+3)) \\
& =S(n)+(3 n+6) \\
& =S(n)+3(n+2)
\end{aligned}
$$

Da 3(n+2) durch 3 teilbar ist, lässt $S(n+3)$ den gleichen Rest wie $S(n)$ bei Division durch 3. Daher treten nur Reste auf, die in unserer ersten Dreiergruppe vorkommen, also die Reste 1 und 2.

Demnach ist der Betrag nie unter den Dreien aufteilbar.

Bemerkung: Interessant ist nun, wie man die entstandene prekäre Situation auslegen soll (Juristen würden sich freuen!). Manuel drückt sich diplomatisch aus: „Wie viel die Enkel bekommen, hängt ganz vom Testament der Oma ab - das ursprüngliche Geburtstagsgeschenk werden sie nämlich nie erhalten, da die Zahlungsbedingung 
niemals eintritt." Das macht den Eindruck, als müsse die Oma zu Lebzeiten gar nichts zahlen. Wadim ist da ganz anderer Meinung: „Das heißt, dass [...] die Oma für den Rest ihres Lebens nach gegebener Vorschrift ihren Enkeln Geld bringen muss. Im ersten Jahr muss sie $66.895 €$ bezahlen."

Auch wir können das Problem nicht abschließend lösen; fest steht nur, dass die Enkel das Geld auf diese Art nie unter sich aufteilen werden können. Die Frage ist, ob sie zwischenzeitlich Teilbeträge aufteilen dürfen. Wenn ja, wird die Oma schnell arm, wenn dies aber nicht der Fall ist, braucht die Oma eigentlich auch nichts zu zahlen (es ist nicht geklärt, ob sie das Geld zwischenzeitlich einem Treuhänder übergibt), und man könnte böswillig vermuten, dass die Oma eine Betrügerin ist, weil sie sich falscher Versprechungen bedient, um sich die Gunst der Kinder zu erschleichen.

L 13.2 Bei dieser Aufgabe kommt es darauf an, von den gegebenen Aussagen die wirklich wichtigen herauszufiltern. Und die einzig wirklich wichtige Aussage ist, dass, nachdem jeder einmal umgeschüttet hat, in jedem Trinkgefäß jeweils die gleiche Menge Flüssigkeit ist wie zu Beginn. Wir vergessen also vorläufig den gesamten Umschüttvorgang und interessieren uns nur für den Anfangs- und den Endzustand. Onkel Heinz hat dann weniger Milch in seinem Glas, stattdessen eine bestimmte Menge Kaffee. Es fehlt in seinem Glas also gegenüber dem Ausgangszustand eine gewisse Menge Milch, und da die Gesamtmenge an Flüssigkeit im Glas gleich geblieben ist, muss die fehlende Menge Milch genauso groß sein wie die neu hinzugekommene Menge Kaffee. Und wo ist diese Milch geblieben? In dem Gemisch, welches Tante Erna in ihrer Tasse hat! Dafür fehlt in Tante Ernas Tasse diese Menge an Kaffee.

Tante Erna hat also genauso viel Milch in ihrer Tasse, wie Onkel Heinz Kaffee in seinem Glas hat. Und das ist unabhängig davon, wie oft diese Prozedur wiederholt wird und ob die Tasse größer ist als das Glas oder nicht, denn diese Größen haben wir bei unseren Überlegungen gar nicht verwendet (vorausgesetzt, die beiden verschütten nichts).

Was vielleicht irritiert, ist, dass Onkel Heinz zu Beginn einen ganzen Löffel Milch in Tante Ernas Tasse schüttet, Tante Erna aber nur einen Löffel Gemisch, also etwas weniger Kaffee als das Volumen eines Löffels. Dadurch könnte man denken, es ist mehr Milch bei Tante Erna als Kaffee bei Onkel Heinz. Aber dabei vergisst man, dass der Rest des Löffels nicht leer bleibt, sondern Milch enthält. Diese Milch wird also wieder aus der Kaffeetasse in das Milchglas zurückgeschüttet und effektiv gar nicht bewegt. Und die Menge ist genau die, die Tante Erna weniger Kaffee zurückschüttet, als sie von Onkel Heinz Milch erhalten hat, denn das Gesamtvolumen des Löffels bleibt konstant.

L 13.3 In der zu zeigenden Gleichung kommen der Umkreisradius und der Inkreisradius des Dreiecks $A B C$ vor. Aus der Schule sollte bekannt sein, dass jedes Dreieck einen Umkreis und einen Inkreis hat und der Mittelpunkt des Umkreises 
gerade der Schnittpunkt der drei Mittelsenkrechten, der Mittelpunkt des Inkreises der Schnittpunkt der Winkelhalbierenden ist.

Selten aber wird in der Schule gelehrt, wie man die Radien dieser Kreise berechnen kann. Hierzu betrachte man die Abbildung 13.1.

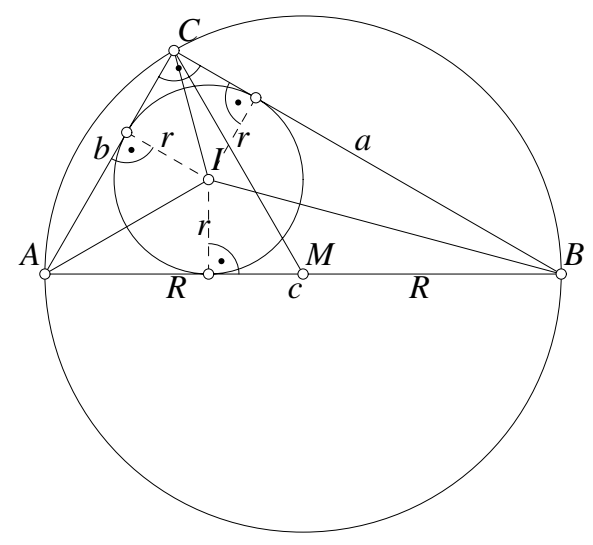

Abbildung 13.1: Zu Aufgabe 3

Im Fall des hier vorliegenden rechtwinkligen Dreiecks ist die Berechnung des Umkreisradius besonders einfach: Der Satz des Thales besagt, dass in einem Kreis jeder Umfangswinkel über einem Durchmesser ein rechter Winkel ist. Auch die Umkehrung dieses Satzes gilt: Ist ein Winkel über einem Durchmesser ein rechter, dann ist er ein Umfangswinkel, liegt also auf dem Kreis über dem Durchmesser.

In unserer Situation gilt also nach der Umkehrung des Satzes des Thales, dass der Punkt $C$ auf dem Kreis mit dem Durchmesser $A B$ liegt. Deswegen ist der Umkreisradius $R$ gerade halb so groß wie $\overline{A B}$ :

$$
R=\frac{1}{2} c .
$$

Für den Inkreisradius $r$ kann man folgende Methode anwenden: Ist $I$ der Inkreismittelpunkt, so zerlegen die Strecken $I A, I B$ und $I C$ das Dreieck $A B C$ in drei Teildreiecke. Diese haben die Flächeninhalte $\frac{1}{2} a r, \frac{1}{2} b r$ und $\frac{1}{2} c r$. Zusammen addieren sich diese Flächen zur Gesamtfläche von $A B C$, die für unser rechtwinkliges Dreieck gleich $\frac{1}{2} a b$ ist. Es gilt also:

$$
\begin{aligned}
\frac{1}{2} a b & =\frac{1}{2} a r+\frac{1}{2} b r+\frac{1}{2} c r \quad \text { bzw } . \\
r & =\frac{a b}{a+b+c} .
\end{aligned}
$$


Damit sind Um- und Inkreisradius bestimmt. Nun kann man ausrechnen:

$$
\begin{array}{rlr}
2(R+r) & =c+\frac{2 a b}{a+b+c} & \\
& =\frac{(a+b) c+c^{2}+2 a b}{a+b+c} & \\
& =\frac{(a+b) c+a^{2}+b^{2}+2 a b}{a+b+c} & \text { (nach Satz des Pythagoras) } \\
& =\frac{(a+b) c+(a+b)^{2}}{a+b+c} & \text { (nach binomischer Formel) } \\
& =\frac{(a+b)(a+b+c)}{a+b+c} & \\
& =a+b . &
\end{array}
$$

Das war zu zeigen.

L 13.4 Zunächst einmal: Vielleicht hätten wir, um deutlich zu machen, dass wir keine Pyromanen sind, die Aufgabe lieber so stellen sollen, dass auf den Waagschalen Trichter angebracht sind, aus denen ein Sandvorrat gleichmäßig auf den Boden rieselt. Aber damit bekäme die ganze Apparatur wohl ein ziemlich unrealistisches Gewicht, das ist auch nicht ideal. Also: Die Kerzen dienen nur als Modell (dass sie rückstandsfrei abbrennen, ist in der Tat fraglich), und von eigenen Versuchen zu

\begin{tabular}{|c|c|c|c|c|c|}
\hline Uhrzeit & $\begin{array}{c}\text { Länge der } \\
\text { einzeln }\end{array}$ & $\begin{array}{l}\text { Kerzen links } \\
\text { insgesamt }\end{array}$ & $\begin{array}{l}\text { Länge der } \\
\text { insgesamt }\end{array}$ & $\begin{array}{l}\text { zen rechts } \\
\text { einzeln }\end{array}$ & \\
\hline 22.00 & $8 \frac{1}{6}, 8 \frac{1}{6}, 8 \frac{2}{3}$ & 25 & 17 & $8 \frac{1}{2}, 8 \frac{1}{2}$ & \\
\hline 4.00 & $2 \frac{1}{6}, 2 \frac{1}{6}, 2 \frac{2}{3}$ & 7 & 5 & $2 \frac{1}{2}, 2 \frac{1}{2}$ & \\
\hline 6.00 & $\frac{1}{6}, \frac{1}{6}, \frac{2}{3}$ & 1 & 1 & $\frac{1}{2}, \frac{1}{2}$ & Läuten! \\
\hline 6.10 & $0,0, \frac{1}{2}$ & $\frac{1}{2}$ & $\frac{2}{3}$ & $\frac{1}{3}, \frac{1}{3}$ & \\
\hline 6.20 & $0,0, \frac{1}{3}$ & $\frac{1}{3}$ & $\frac{1}{3}$ & $\frac{1}{6}, \frac{1}{6}$ & Läuten! \\
\hline 6.30 & $0,0, \frac{1}{6}$ & $\frac{1}{6}$ & 0 & 0,0 & \\
\hline 6.40 & $0,0,0$ & 0 & 0 & 0,0 & $\begin{array}{l}\text { (Dauer-) } \\
\text { Läuten! }\end{array}$ \\
\hline
\end{tabular}
Hause wird dringend abgeraten.

Eine Lösung des Problems ist die folgende: Um 22 Uhr stelle man auf die linke Seite der Waage eine Kerze von 8 2/3 cm Länge und zwei Kerzen von $81 / 6 \mathrm{~cm}$ Länge; auf die rechte Seite kommen zwei Kerzen mit je $81 / 2 \mathrm{~cm}$ Länge. Dann ergibt sich folgendes Bild im Laufe der Nacht:

Die Weckzeiten werden also erreicht. Und dazwischen wird auch nicht geweckt, denn von 22.00 Uhr bis 6.10 Uhr brennt auf der linken Seite eine Kerze mehr als auf der 
rechten, daher neigt sich die Waage beständig nach rechts, so dass es nur zu genau einem Zeitpunkt zum Gleichgewicht kommen kann. Zwischen 6.10 Uhr und 6.30 Uhr ist es umgekehrt, da brennt auf der rechten Seite eine Kerze mehr als auf der linken, also neigt sich die Waage beständig nach links, und danach neigt sie sich wieder nach rechts, da nur noch links eine Kerze brennt.

Nun kann man sich fragen, ob man eventuell mit noch weniger Kerzenmaterial auskommt. Das ist zu verneinen, wie man sich klarmachen kann, wenn man die Aufgabe rückwärts angeht:

- Bis um 6.40 Uhr muss mindestens eine Kerze gebrannt haben. Ohne Beschränkung der Allgemeinheit dürfen wir annehmen, dass sie auf der linken Seite gestanden hat. (Um 22 Uhr muss sie also eine Länge von $82 / 3 \mathrm{~cm}$ gehabt haben.)

- Um 6.20 Uhr muss Gleichgewicht herrschen. Also muss dann auf der rechten Seite $1 / 3 \mathrm{~cm}$ Kerze vorhanden sein, um die erstgenannte Kerze auszugleichen (theoretisch eventuell auch mehr, da wir hier ja noch nicht untersuchen, ob sich so tatsächlich eine Lösung ergibt). Und da die Waage kurz vor 6.40 Uhr nach links geneigt ist (dort brennt die entscheidende Kerze) und in der Zeit zwischen 6.20 Uhr und 6.40 Uhr kein Gleichgewicht herrschen darf, muss sie sich um 6.20 Uhr (vom Gleichgewicht aus) nach links neigen. Das bedeutet, dass auf der rechten Seite mindestens eine Kerze mehr stehen muss als auf der linken, also mindestens zwei.

- Um 6.00 Uhr ist auf der rechten Seite nach den vorherigen Überlegungen (mindestens) $1 / 3 \mathrm{~cm}+2 \cdot 1 / 3 \mathrm{~cm}=1 \mathrm{~cm}$ Kerze, auf der linken $2 / 3 \mathrm{~cm}$. Also muss, um Gleichgewicht zu erhalten, auf der linken Seite noch $1 / 3 \mathrm{~cm}$ Kerze zusätzlich vorhanden sein. Und mit einem entsprechenden Argument wie eben sieht man ein, dass nun auf der linken Seite mindestens drei Kerzen stehen müssen. Demnach sind um $22 \mathrm{Uhr}$ auf der linken Seite (mindestens) $1 \mathrm{~cm}+3 \cdot 8 \mathrm{~cm}=25 \mathrm{~cm}$ Kerze, auf der rechten $1 \mathrm{~cm}+2 \cdot 8 \mathrm{~cm}=17 \mathrm{~cm}$. Und genau das hatten wir oben.

Die Lösung ist aber nicht ganz eindeutig: Fängt man mit 8 1/12, $83 / 12$ und 8 2/3 zu 8 5/12 und 8 7/12 Zentimetern an, so ist dies auch eine Lösung, die sich nur darin von der ersten unterscheidet, dass sich die Waage zwischen 6.05 und 6.15 Uhr sowie 6.25 und 6.35 Uhr nicht bewegt, weil dann auf beiden Seiten gleich viele Kerzen brennen.

Wenn die Waage empfindlich gegen große Ausschläge ist, kann man auf die rechte Seite noch eine weitere Kerze mit z. B. $75 / 6 \mathrm{~cm}$ Länge stellen. Dann beträgt der Gewichtsunterschied der beiden Seiten maximal das Gewicht von einem Sechstel Zentimeter Kerze. 


\section{Lösungen zu Aufgabenblatt 14}

L 14.1 Laura irrt sich. Es kann durchaus passieren, dass keine drei Stäbe zu einem Dreieck zusammenlegbar sind.

Wenn man drei Stäbe zu einem Dreieck zusammenlegen kann, dann erfüllen die Längen der Stäbe gewisse Bedingungen (die so genannten Dreiecksungleichungen). Nämlich: Kein Stab darf länger sein als die beiden anderen Stäbe zusammen. Denn machen wir je ein Ende der kürzeren Stäbe an den Enden des längsten fest, so muss man ja die freien Enden der kürzeren Stäbe noch zusammenheften können.

Nun können wir allerdings Stäbe basteln, die nicht die von Laura genannte Eigenschaft besitzen:

Wir beginnen mit zwei beliebigen Stäben. Den dritten Stab machen wir ein bisschen länger, als die ersten zwei zusammen lang sind. Den vierten Stab machen wir wiederum länger, als die zwei größten der bisherigen drei Stäbe zusammen lang sind, und damit ist der vierte länger als die Gesamtlänge zweier beliebiger der ersten drei. Das setzen wir nun fort. Der fünfte Stab wird wieder länger gemacht, als die zwei größten der ersten vier zusammen lang sind, der sechste wieder länger, ...

Auf die Weise können also nie drei Stäbe zu einem Dreieck zusammengelegt werden. Bemerkungen: Wenn wir ausgeartete Dreiecke (also Dreiecke, deren Flächeninhalt null ist, oder anders gesagt Dreiecke, bei denen ein Eckpunkt auf derjenigen Seite liegt, die die anderen zwei Eckpunkte verbindet) nicht zulassen, so reicht es sogar aus, den jeweils nächsten Stab genau so lang zu machen, wie die größten zwei der übrigen Stäbe zusammen lang sind.

Praktisch gesehen wird aber der von uns konstruierte Fall nie eintreten. Beginnt man nämlich mit zwei Stäben der Länge $1 \mathrm{~cm}$, so hat der nächste die Länge $2 \mathrm{~cm}$, der vierte die Länge $3 \mathrm{~cm}$, dann $5 \mathrm{~cm}, 8 \mathrm{~cm}, 13 \mathrm{~cm}, 21 \mathrm{~cm}, \ldots$

Der 37. Stab hätte dann eine Länge von $241,57817 \mathrm{~km}$. Also viel zu lang!

Wenn man genau überlegt, wird man feststellen, dass unser Kriterium (der längste Stab muss länger sein als die Gesamtlänge zweier anderer) auch notwendig dafür ist, dass man keine Dreiecke konstruieren kann. Wenn man es also genau nimmt, hat Laura, praktisch gesehen, doch Recht.

L 14.2 Die beiden Kneipen sind 1000 Meter voneinander entfernt. Die einzige physikalische Formel, die wir hier brauchen, ist

$$
v \cdot t=s
$$

Sie besagt: Wenn man sich eine Zeit $t$ [Minuten] lang mit einer Geschwindigkeit $v$ [Meter/Minute] vorwärts bewegt, so legt man eine Strecke von $s$ [Metern] zurück. 
Herr Schluckspecht legt mit seiner Geschwindigkeit $v_{1}$ in den ersten $t$ Minuten bis zum Treffen $200 \mathrm{~m}$ mehr zurück als Herr Suffkopp, der mit einer Geschwindigkeit $v_{2}$ läuft:

$$
v_{1} t=v_{2} t+200 .
$$

Bis zu ihrem Treffpunkt ist jeder genau den Teil der Strecke gelaufen, den der andere nicht gelaufen ist, daher haben sie zusammen genau einmal die Entfernung $s$ zwischen den beiden Kneipen zurückgelegt:

$$
v_{1} t+v_{2} t=s .
$$

Diese Gleichungen addieren wir und formen sie äquivalent um:

$$
\begin{aligned}
& 2 v_{1} t+v_{2} t=v_{2} t+s+200 \quad \mid-v_{2} t \\
& \Leftrightarrow \quad 2 v_{1} t=s+200 \quad \mid: 2 \\
& \Leftrightarrow \quad v_{1} t=\frac{s+200}{2}
\end{aligned}
$$

Dies setzen wir in die nach $v_{2} t$ umgestellte Gleichung (14.1) ein:

$$
v_{2} t \stackrel{(14.1)}{=} v_{1} t-200 \stackrel{(14.3)}{=} \frac{s+200}{2}-200=\frac{s-200}{2} .
$$

(Auf das Gleichheitszeichen schreiben wir manchmal auch die Nummer der angewandten Gleichung.)

Nach dem Treffen der beiden Kampfhähne braucht Herr Schluckspecht für den restlichen Weg $s-v_{1} t \stackrel{(14.2)}{=} v_{2} t$ mit halber Geschwindigkeit $\frac{v_{1}}{2}$ noch 8 min:

$$
\frac{v_{1}}{2} \cdot 8=v_{2} t
$$

Herr Suffkopp hingegen braucht für $s-v_{2} \stackrel{(14.2)}{=} v_{1} t$ Meter in seiner halben Geschwindigkeit $\frac{v_{2}}{2}$ noch 18 min:

$$
\frac{v_{2}}{2} \cdot 18=v_{1} t
$$

Jetzt formen wir (14.5) und (14.6) jeweils nach $\frac{v_{1}}{v_{2}}$ um:

$$
\frac{v_{1}}{v_{2}}=\frac{t}{4} \quad \text { und } \quad \frac{v_{1}}{v_{2}}=\frac{9}{t}
$$

und erhalten durch Gleichsetzen:

$$
\begin{aligned}
\frac{t}{4} & =\frac{9}{t} & \mid \cdot 4 t \\
\Leftrightarrow & t^{2} & =36 .
\end{aligned}
$$


Lösungen zu Aufgabenblatt 14

Von den beiden Lösungen $t=6$ oder $t=-6$ der quadratischen Gleichung ist als Zeitangabe nur die positive möglich, die beiden Freunde begegnen sich also nach

$$
t=6
$$

Minuten.

Ersetzen wir in Gleichung (14.5) die rechte Seite noch durch (14.4):

$$
\frac{v_{1}}{2} \cdot 8=\frac{s-200}{2},
$$

so haben wir zusammen mit (14.3) dann zwei Gleichungen, in denen nur noch die zwei unbekannten Variablen $s$ und $v_{1}$ vorkommen ( $t$ ist ja bereits bekannt).

Da beide Seiten von (14.3) positiv sind (dies ist ja gerade der Weg, für den Herr Suffkopp 18 Minuten braucht, also kann er nicht null oder gar negativ sein), können wir die letzte Gleichung durch (14.3) teilen:

$$
\frac{\frac{v_{1}}{2} \cdot 8}{v_{1} t}=\frac{\frac{s-200}{2}}{\frac{s+200}{2}} ;
$$

gekürzt und $t=6$ nach (14.7) eingesetzt:

$$
\begin{aligned}
& \frac{4}{6}=\frac{s-200}{s+200} \quad \mid \cdot(s+200) \\
& \Leftrightarrow \quad \frac{2}{3} s+\frac{2}{3} \cdot 200=s-200 \quad \mid-s-\left(\frac{2}{3} \cdot 200\right) \\
& \Leftrightarrow \quad-\frac{1}{3} s=-\frac{5}{3} \cdot 200 \quad \mid \cdot(-3) \\
& \Leftrightarrow \quad s=\underline{\underline{5 \cdot 200=1000}} .
\end{aligned}
$$

Da wir zwischendurch auch mal eine Wurzel gezogen haben (keine Äquivalenzumformung!), machen wir noch eine Probe, bevor wir $s=1000$ als gesichertes Ergebnis ansehen. Dazu müssen wir aber noch $v_{1}$ und $v_{2}$ berechnen.

Aus Formel (14.3) erhalten wir: $v_{1}=\frac{1200}{2 \cdot 6}=100$, Formel (14.4) sagt: $v_{2}=\frac{800}{12}=\frac{200}{3}$. Nun setzen wir alles, was wir bestimmt haben $\left(t=6, s=1000, v_{1}=100\right.$ und $v_{2}=\frac{200}{3}$ ), in die vier Ausgangsgleichungen (14.1), (14.2), (14.5) und (14.6) ein und hoffen, dass wir nur wahre Aussagen erhalten:

$$
\begin{array}{r}
v_{1} t=100 \cdot 6=600=400+200=\frac{200}{3} \cdot 6+200=v_{2} t+200 \\
v_{1} t+v_{2} t=100 \cdot 6+\frac{200}{3} \cdot 6=600+400=1000=s \\
\frac{v_{1}}{2} \cdot 8=50 \cdot 8=400=\frac{200}{3} \cdot 6=v_{2} t \\
\frac{v_{2}}{2} \cdot 18=\frac{100}{3} \cdot 18=600=100 \cdot 6=v_{1} t .
\end{array}
$$

Die Entfernung der beiden Kneipen beträgt also 1000 Meter. 
L 14.3 Es lassen sich alle natürlichen Zahlen in der gegebenen Form darstellen. Wir müssen Summen und Differenzen von Quadraten aufeinander folgender natürlicher Zahlen betrachten.

Wählen wir eine beliebige Zahl $n$ und bilden die Differenz

$$
n^{2}-(n+1)^{2}=n^{2}-n^{2}-2 n-1=-2 n-1,
$$

so hängt diese nur noch linear von $n$ ab, aber nicht mehr quadratisch. Bilden wir nun die Differenz zweier aufeinander folgender derartiger Differenzen:

$$
\begin{aligned}
n^{2}-(n+1)^{2} & -\left((n+2)^{2}-(n+3)^{2}\right) \\
& =-2 n-1-(-2(n+2)-1) \\
& =-2 n-1+2 n+4+1 \\
& =4 .
\end{aligned}
$$

Also hat jede natürliche Zahl $n$ die schöne Eigenschaft $n^{2}-(n+1)^{2}-(n+2)^{2}+(n+3)^{2}=4$.

Bekanntlich können wir jede natürliche Zahl $p$ in der Form $p=r+v \cdot 4$ darstellen, wobei $v \in \mathbb{N}$ und $r=0,1,2$ oder 3 ist, also $p=r+(4+\ldots+4)$.

Dies benutzen wir, um $p$ als Summe $\pm 1^{2} \pm \ldots \pm m^{2}$ zu schreiben.

Aus der Beispielliste können wir $r$ entnehmen (für $r=0$ nehmen wir einfach eine leere Summe), und wie wir die Summe $(4+\cdots+4)$ geeignet dazuaddieren können, haben wir oben gesehen. Damit erhalten wir für $p$ folgende Zerlegung:

$$
\begin{aligned}
\text { für } r=0: p= & \left(1^{2}-2^{2}-3^{2}+4^{2}\right)+\ldots \\
& +\left((4 v-3)^{2}-(4 v-2)^{2}-(4 v-1)^{2}+(4 v)^{2}\right) \\
\text { für } r=1: p= & 1^{2}+\left(2^{2}-3^{2}-4^{2}+5^{2}\right)+\ldots \\
& +\left((4 v-2)^{2}-(4 v-1)^{2}-(4 v)^{2}+(4 v+1)^{2}\right) \\
\text { für } r=2: p= & -1^{2}-2^{2}-3^{2}+4^{2}+\left(5^{2}-6^{2}-7^{2}+8^{2}\right)+\ldots \\
& +\left((4 v+1)^{2}-(4 v+2)^{2}-(4 v+3)^{2}+(4 v+4)^{2}\right) \\
\text { für } r=3: p= & -1^{2}+2^{2}+\left(3^{2}-4^{2}-5^{2}+6^{2}\right)+\ldots \\
& +\left((4 v-1)^{2}-(4 v)^{2}-(4 v+1)^{2}+(4 v+2)^{2}\right)
\end{aligned}
$$

Bemerkung: Wie schon in den ersten Beispielen ersichtlich, ist die Darstellung nicht eindeutig (z. B. $4=-1^{2}-2^{2}+3^{2}=1^{2}-2^{2}-3^{2}+4^{2}$ ).

L 14.4 Wie in der Aufgabenstellung schon beschrieben, findet man für die meisten Paare $(m, n)$ natürlicher Zahlen in einem $m \times n$-Rechteck einen gesuchten Streckenzug. Beginnt man mit kleinen $m$ und $n$, so schlägt die Suche nach einem solchen Streckenzug nur bei den Paaren $(2,2),(2,4),(4,2),(4,4),(2,6)$ usw. fehl. Dies legt folgende Vermutung nahe: 
Es gibt in einem $m \times n$-Rechteck genau dann einen geschlossenen, überschneidungsfreien Weg entlang der Gitterkanten, der jeden Gitterpunkt genau einmal berührt, wenn wenigstens eine der Zahlen $m$ oder $n$ ungerade ist.

Wie beweist man das?

Nun, dazu müssen zwei Dinge gezeigt werden: zum einen, dass es einen erlaubten Streckenzug gibt, falls $m$ oder $n$ ungerade ist, zum anderen, dass es keinen solchen gibt, wenn beide Zahlen gerade sind.

Ersteres kann man (einfach) durch die Angabe eines gesuchten Streckenzuges tun. Hierzu sei zum Beispiel $m$ ungerade (andernfalls muss $n$ ungerade sein und die Skizze muss nur um $90^{\circ}$ gedreht werden). Dann kann man den in Abbildung 14.1 gezeigten, alle Bedingungen erfüllenden Streckenzug in ein $m \times n$-Rechteck zeichnen.

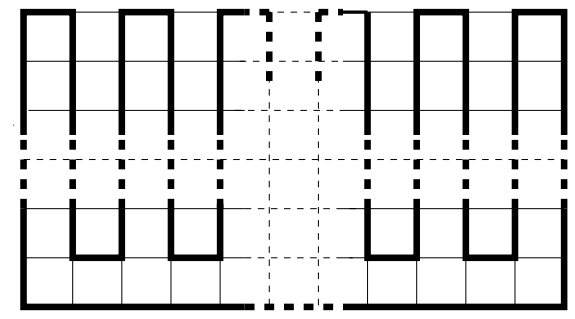

Abbildung 14.1: Möglicher Streckenzug, wenn $m$ oder/und $n$ ungerade sind

Der zweite Teil des Beweises ist etwas schwieriger (ganz allgemein ist es meist komplizierter zu beweisen, dass etwas nicht geht, als zu zeigen, dass etwas geht).

Seien also $m$ und $n$ gerade. Dann gibt es $n+1$ waagerechte und $m+1$ senkrechte Gitterlinien im Rechteck und deshalb $(n+1)(m+1)$ Gitterpunkte, die ein erlaubter Streckenzug alle genau einmal treffen müsste. Da ein solcher Streckenzug geschlossen ist, müsste der Streckenzug also auch aus genau $(n+1)(m+1)$ verschiedenen Einheitsstrecken bestehen. Nun sind aber $m$ und $n$ gerade, also $m+1$ und $n+1$ beide ungerade. Die Länge eines erlaubten Streckenzuges wäre also ungerade.

Das kann aber nicht sein!

Warum? Weil jeder geschlossene Streckenzug entlang der Gitterkanten eine gerade Länge haben muss.

Betrachtet man nämlich den Streckenzug von einem beliebigen Punkt des Streckenzuges aus und verfolgt ihn Schritt für Schritt, so verläuft er $l$-mal nach links, $r$-mal nach rechts, o-mal nach oben und $u$-mal nach unten. Letztlich soll der Streckenzug, da er geschlossen ist, aber wieder beim Ausgangspunkt ankommen. Deswegen muss er genauso oft nach rechts wie nach links gehen, also ist $l=r$, und genauso oft nach oben wie nach unten, also ist $o=u$. Damit ist die Länge des Streckenzuges $r+l+o+u=2 \cdot r+2 \cdot o=2 \cdot(r+o)$ gerade. 
Damit kann es also im Fall, dass sowohl $m$ als auch $n$ gerade sind, keinen erlaubten Streckenzug geben.

Ist nun aber $m$ oder $n$ ungerade, so gibt es, wie oben gesehen, wenigstens einen, aber meist noch viel mehr erlaubte Streckenzüge. In oben gezeichnetem Beispiel schließt dieser gerade eine Fläche von $m n-\frac{m-1}{2}(n-1)=\frac{(m+1)(n+1)}{2}-1$ (Kästchen) ein. Ist diese Fläche für einen anderen Streckenzug größer oder kleiner?

Um das herauszufinden, zerlegen wir die eingeschlossene Fläche eines erlaubten Streckenzuges schrittweise in die einzelnen Einheitsquadrate. In einem Schritt starten wir dabei bei einem Randpunkt unseres Streckenzuges und zeichnen eine Einheitsstrecke entlang der Gitterlinien in das Innere der eingeschlossenen Fläche hinein. Da jeder Gitterpunkt auf dem Streckenzug liegt, landen wir dabei wieder auf dem Rand des Streckenzuges und haben somit die Fläche in zwei Teile geteilt. Die Gesamtzahl der Randpunkte der beiden Teile ist dabei um zwei größer als die Zahl der Randpunkte des Ausgangsteiles, da die beiden Endpunkte der zum Zerteilen gezogenen Einheitsstrecke Randpunkte beider neu entstandenen Teile sind.

Dieses Zerteilen kann man offenbar so lange fortführen, wie es „innere Kanten“ in einem der Flächenteile gibt. Die Anzahl dieser inneren Kanten nimmt aber in jedem Schritt um eins ab. Irgendwann, sagen wir nach $N$ Schritten, gibt es also keine inneren Kanten mehr, das heißt es gibt nur noch Einheitsquadrate. Da bei jedem Teilungsschritt ein Flächenteil mehr entsteht, hat man also schließlich $N+1$ Einheitsquadrate mit insgesamt $(m+1)(n+1)+2 N$ Randpunkten. Es gilt also

$$
\begin{aligned}
4(N+1) & =(m+1)(n+1)+2 N \quad \text { bzw. } \\
N & =\frac{(m+1)(n+1)}{2}-2 .
\end{aligned}
$$

Die vom Streckenzug eingeschlossene Fläche ist also $N+1=\frac{(m+1)(n+1)}{2}-1$, und dies ist unabhängig von der Form des Streckenzugs.

Vielleicht kennt der eine oder andere folgenden hübschen Satz von Pick über die Fläche von Gitterpunktpolygonen:

Hat man einen geschlossenen Streckenzug (nicht unbedingt entlang der Gitterlinien), dessen Eckpunkte Gitterpunkte sind, und liegen genau $r$ Gitterpunkte auf dem Rand des Streckenzuges und genau $i$ Gitterpunkte im Inneren der eingeschlossenen Fläche, so hat die eingeschlossene Fläche $A$ die Größe $A=i+\frac{r}{2}-1$.

Mit diesem Satz wäre obige Aussage auch schnell zu beweisen, denn für einen erlaubten Streckenzug ist $r=(m+1)(n+1)$ und $i=0$. 


\section{Lösungen zu Aufgabenblatt 15}

L 15.1 Vor dem Wechsel seien in der Klasse 8a $m_{a}$ Mädchen und $j_{a}$ Jungen, in der $8 \mathrm{~b} m_{b}$ Mädchen und $j_{b}$ Jungen, wobei $m_{a}>j_{a} \geq 1$ (es wechseln ja ein Mädchen und ein Junge aus der 8a) und $0 \leq m_{b}<j_{b}$.

Der Mädchenanteil in der 8a ist also $\frac{m_{a}}{m_{a}+j_{a}}$ und der in der $8 \mathrm{~b}$ ist $\frac{m_{b}}{m_{b}+j_{b}}$.

Nun verlassen ein Junge und ein Mädchen die $8 \mathrm{a}$ und besuchen fortan die 8b. Damit verändert sich der Mädchenanteil in der $8 \mathrm{a}$ zu $\frac{m_{a}-1}{\left(m_{a}-1\right)+\left(j_{a}-1\right)}=\frac{m_{a}-1}{m_{a}+j_{a}-2}$ und in der $8 \mathrm{~b} \mathrm{zu} \frac{m_{b}+1}{\left(m_{b}+1\right)+\left(j_{b}+1\right)}=\frac{m_{b}+1}{m_{b}+j_{b}+2}$. Wir wollen zeigen, dass sich durch den Wechsel der Mädchenanteil in beiden Klassen erhöht, also

$$
\text { (a) } \frac{m_{a}}{m_{a}+j_{a}}<\frac{m_{a}-1}{m_{a}+j_{a}-2} \quad \text { und } \quad(b) \frac{m_{b}}{m_{b}+j_{b}}<\frac{m_{b}+1}{m_{b}+j_{b}+2} \text {. }
$$

$\mathrm{Zu}(a)$ : Wegen $m_{a}>j_{a} \geq 1$ gilt: $m_{a}+j_{a}, m_{a}+j_{a}-2>0$ und somit:

$$
\begin{aligned}
\frac{m_{a}}{m_{a}+j_{a}} & <\frac{m_{a}-1}{m_{a}+j_{a}-2} \\
\Leftrightarrow m_{a}\left(m_{a}+j_{a}-2\right) & <\left(m_{a}+j_{a}\right)\left(m_{a}-1\right) \\
\Leftrightarrow m_{a}^{2}+m_{a} j_{a}-2 m_{a} & <m_{a}^{2}-m_{a}+m_{a} j_{a}-j_{a} \\
\Leftrightarrow-m_{a} & <-j_{a} \\
\Leftrightarrow m_{a} & >j_{a} .
\end{aligned}
$$

Da die letzte Ungleichung nach Voraussetzung wahr ist, ist auch Ungleichung $(a)$ wahr.

$\mathrm{Zu}(b)$ : Wegen $0 \leq m_{b}<j_{b}$ gilt: $m_{b}+j_{b}, m_{b}+j_{b}+2>0$ und somit:

$$
\begin{aligned}
\frac{m_{b}}{m_{b}+j_{b}} & <\frac{m_{b}+1}{m_{b}+j_{b}+2} \\
\Leftrightarrow m_{b}\left(m_{b}+j_{b}+2\right) & <\left(m_{b}+j_{b}\right)\left(m_{b}+1\right) \\
\Leftrightarrow m_{b}^{2}+m_{b} j_{b}+2 m_{b} & <m_{b}^{2}+m_{b}+m_{b} j_{b}+j_{b} \\
\Leftrightarrow m_{b} & <j_{b} .
\end{aligned}
$$

Da die letzte Ungleichung nach Voraussetzung wahr ist, ist auch Ungleichung (b) wahr.

L 15.2 Entsprechend der Aufgabenstellung darf man zum Bilden der sechsstelligen Zahlen genau die vier Ziffern 2, 3, 5 und 8 verwenden. Wie viele dieser Zahlen haben eine 2 als Einerziffer? 
Für eine solche Zahl bleiben noch vier Möglichkeiten für die Zehnerziffer, vier für die Hunderterziffer, ... und vier für die Hunderttausenderziffer. Das sind insgesamt $4 \cdot 4 \cdot 4 \cdot 4 \cdot 4=4^{5}$ Möglichkeiten, also gibt es $4^{5}$ Zahlen, deren Einerziffer eine 2 ist. Natürlich gibt es genauso viele Zahlen, nämlich $4^{5}$, deren Einerziffer eine 3 ist, eine 5 ist bzw. eine 8 ist.

Es ändert sich auch nichts an dieser Anzahl, wenn man nach den Zahlen fragt, die eine gewisse Ziffer an der Zehner-, Hunderter- oder irgendeiner anderen festen Stelle haben - stets hat man für die übrigen fünf Stellen jeweils vier Ziffern zur Auswahl. Deswegen ergibt sich beim Addieren aller entsprechend der Aufgabe gebildeten Zahlen:

$$
\begin{aligned}
\left(4^{5} \cdot 2+4^{5} \cdot 3+4^{5} \cdot 5+4^{5} \cdot 8\right)+ & \\
\left(4^{5} \cdot 20+\ldots+4^{5} \cdot 80\right)+ & \\
\ldots+ & \\
\left(4^{5} \cdot 2 \cdot 10^{5}+\ldots+4^{5} \cdot 8 \cdot 10^{5}\right)= & 4^{5} \cdot(2+3+5+8) \cdot \\
& (1+10+\ldots+100000) \\
= & 4^{5} \cdot 18 \cdot 111111 \\
= & 2047997,952 .
\end{aligned}
$$

L 15.3 Es ist nicht möglich, dass der zweite und der dritte Freund beide ihre Wette gewonnen oder verloren haben.

Begründung: Angenommen, der dritte Freund gewinnt seine Wette. Das geht nur, wenn alle drei beim ersten Wurf getroffen haben. Damit hat allerdings der erste Freund offensichtlich seine Wette verloren. Er hat aber einen Korb geworfen, daher ist die Wette des zweiten Freundes auch verloren. Also können der zweite und dritte Freund nicht beide gewinnen.

Nun ist zu prüfen, ob sie denn beide verlieren können. Dazu sei angenommen, dass der zweite Freund seine Wette verliert. Das geht nur ${ }^{1}$, wenn der erste Freund trifft

\footnotetext{
${ }^{1} \mathrm{Da}$ es wohl nicht allen Korrespondenzzirkel-Teilnehmern bekannt ist, erläutern wir kurz, wie in der Logik mit Sätzen vom Typ: „Wenn $A$, dann $B$ “ bzw. (was äquivalent ist): „Aus $A$ folgt $B$ “ umzugehen ist: Wenn $A$ richtig ist, haben wir keine Probleme. Dann hängt es allein von $B \mathrm{ab}$, ob die Aussage richtig ist: Ist $B$ falsch, ist es auch die Aussage, ist $B$ richtig, stimmt die Aussage ebenfalls. Wenn nun $A$ falsch ist, ist die Aussage zumindest nicht falsch, denn über diesen Fall wurde ja gar nichts behauptet. Da man aber davon ausgeht, dass eine Aussage entweder falsch oder wahr sein muss (Satz vom ausgeschlossenen Dritten, als lateinischer Terminus: „tertium non datur"), ist die Aussage dann als wahr zu werten (und zwar unabhängig von der Richtigkeit von $B$ !).

In einem anderen Umfeld ist dies vielleicht einsichtiger: Angenommen, die Eltern versprechen: "Wenn du eine Eins in Mathe bekommst, gehen wir am Wochenende ins Theater/Kino". Bekommt man dann keine Eins, so kann man den Eltern nicht vorwerfen, dass sie ihr Versprechen gebrochen haben, egal, ob sie einen dann vielleicht doch aus Gnade mit ins Theater/Kino nehmen oder nicht. Meckern kann man nur dann, wenn man die Eins bekommen hat und sich die Eltern dennoch drücken.
} 
Lösungen zu Aufgabenblatt 15

und seine Wette verliert. Dann müssen aber auch die beiden anderen getroffen haben, mithin muss der dritte Freund seine Wette gewinnen - sie können also auch nicht beide verlieren.

L 15.4 Der Flächeninhalt des Rechtecks beträgt $45 \mathrm{~cm}^{2}$. Die Zahl 45 soll in eine Summe von möglichst vielen natürlichen Summanden zerlegt werden. (Alle Angaben von Seitenlängen und Flächeninhalten beziehen sich auf die Einheiten $\mathrm{cm}$ und $\mathrm{cm}^{2}$.) Bilden wir die Summe $1+2+3+4+5+6+7+8+9$, so erhalten wir gerade den Wert 45. Und tatsächlich können wir das große Rechteck in neun nicht deckungsgleiche Rechtecke mit diesen Flächeninhalten und ganzzahligen Seitenlängen zerlegen:

\begin{tabular}{|c|c|c|}
\hline \multicolumn{3}{|c|}{9} \\
\hline 1 & \multicolumn{2}{|c|}{8} \\
\hline 2 & \multicolumn{2}{c|}{7} \\
\hline \multicolumn{2}{|c|}{3} & \multicolumn{2}{c|}{6} \\
\hline \multicolumn{2}{|c|}{4} & 5 \\
\hline
\end{tabular}

Die Flächeninhalte 4 und 6 dürfen aber beispielsweise zweimal auftreten, da diese Zahlen verschiedene Produktzerlegungen $4=1 \cdot 4$ und $4=2 \cdot 2,6=1 \cdot 6$ und $6=2 \cdot 3$ besitzen. Es gibt deshalb jeweils zwei nicht deckungsgleiche Rechtecke mit den Flächeninhalten 4 und 6. Die Flächeninhalte 1,2, 3, 5, 7 können aber nur einmal vorkommen. Bilden wir nun unter diesen Voraussetzungen die Summe mit möglichst kleinen Summanden, so erhalten wir:

$$
\begin{gathered}
1+2+3+4+4+5+6+6+7=38, \\
1+2+3+4+4+5+6+6+7+8=46 .
\end{gathered}
$$

Eine Summe mit zehn Summanden mit der geforderten Eigenschaft ist also stets größer als 45 .

Daher besteht jede Zerlegung des Rechtecks aus maximal neun kleinen Rechtecken. Unser Beispiel ist also eine Lösung.

Bemerkung: Die Summe $2+3+4+4+5+6+6+7+8=45$ ist eine weitere Darstellung der Zahl 45 mit der geforderten Eigenschaft. Eine passende Zerlegung ist die folgende:

\begin{tabular}{|l|l|l|l|l|l|}
\hline \multicolumn{3}{|c|}{7} & & \\
\hline \multirow{3}{*}{8} & \multirow{2}{*}{6} & 4 & 2 & 4 & \multirow{2}{*}{5} \\
\cline { 3 - 4 } & & 3 & & \\
\cline { 2 - 4 } & \multicolumn{3}{|c|}{6} & & \\
\hline
\end{tabular}




\section{Lösungen zu Aufgabenblatt 16}

L 16.1 Die Kinder können zu ihren Müttern gelangen, ohne einzubrechen. Hierzu muss Beate aber - wie in den Abbildungen eingezeichnet - Anton und Christoph umkurven.
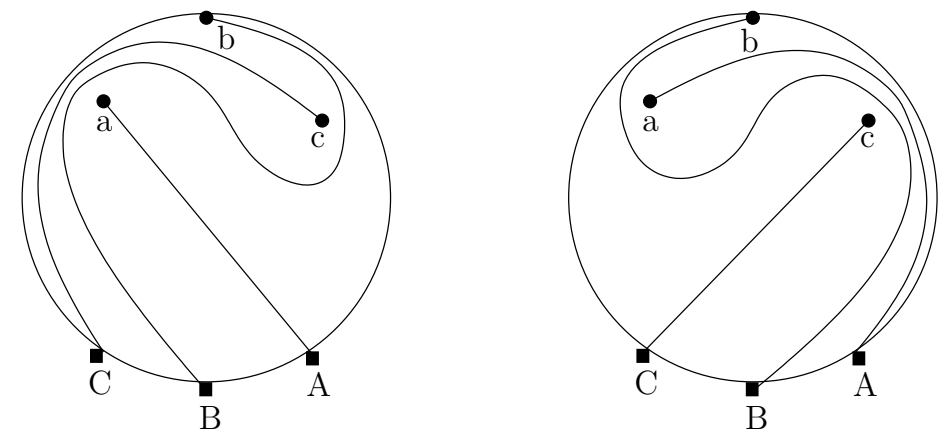

L 16.2 Nach Voraussetzung gilt $p>5$. Damit sind die beiden Primzahlen ungerade.

Für ihre Summe $S$ gilt nun: $S=p+(p+2)=2 \cdot(p+1)$. Da $p$ ungerade ist, ist $(p+1)$ gerade. Demnach ist $S$ durch $2 \cdot 2$ teilbar.

Von den drei aufeinander folgenden Zahlen $p, p+1$ und $p+2$ ist genau eine durch 3 teilbar. Da $p$ und $p+2$ Primzahlen größer als 5 sind, können die beiden nicht durch 3 teilbar sein. Deshalb muss $p+1$ durch 3 teilbar sein. Somit ist ein dritter Faktor von $S$ gefunden.

Es gilt: $S=2 \cdot 2 \cdot 3 \cdot n=12 \cdot n$ mit einem $n \in \mathbb{N}$.

Wegen $p>5$ ist $S>12$, folglich ist $n>1$.

Die Summe lässt sich also zerlegen in $p+(p+2)=2 \cdot 2 \cdot 3 \cdot n$ mit $n \in \mathbb{N}, n>1$.

L 16.3 Wenn man nach Süden oder nach Norden geht, bewegt man sich entlang eines Meridians (Längengrads). Geht der Abenteurer nach Süden, so bleibt er auf dem Meridian seiner Hütte. Wendet er sich Richtung Osten, so verlässt er ihn und landet auf einem weiteren Meridian, auf dem er dann Richtung Norden zu seinem Ausgangspunkt zurück wandert. Dies ist aber nur dann möglich, wenn die beiden Meridiane (der, auf dem er startet, und der, auf dem er zurückgeht) sich im Ausgangspunkt schneiden oder identisch sind.

Der erste Fall tritt nur ein, wenn die Hütte am Nord- oder Südpol steht. Am Südpol aber kann der Abenteurer nicht starten, da er von dort aus nicht nach Süden gehen kann. Beginnt der Abenteurer jedoch seine Expedition am Nordpol, so endet diese 
auch wieder dort, da sich beim Wandern nach Osten der Abstand zum Nordpol nicht ändert.

Der zweite Fall tritt genau dann ein, wenn unser Abenteurer auf seinem Weg nach Osten die Erde $n$-mal umrundet ( $n$ ist dabei eine natürliche Zahl), das heißt, dass der durchwanderte Breitenkreis gerade den Umfang 10/ $n \mathrm{~km}$ hat. Solche findet man nur in der Nähe der Pole. Möchte der Wanderer die Oststrecke in der Nähe des Nordpols bewältigen, so muss sein Weg nördlicher liegen als im ersten Fall. Dazu müsste seine Hütte nördlicher als der Nordpol liegen, was natürlich nicht möglich ist. In der Nähe des Südpols gibt es andererseits zu jedem $n$ einen Breitenkreis, der genau $10 / n \mathrm{~km}$ als Umfang hat und den er durch seine Wanderroute erreicht, siehe Abbildung.

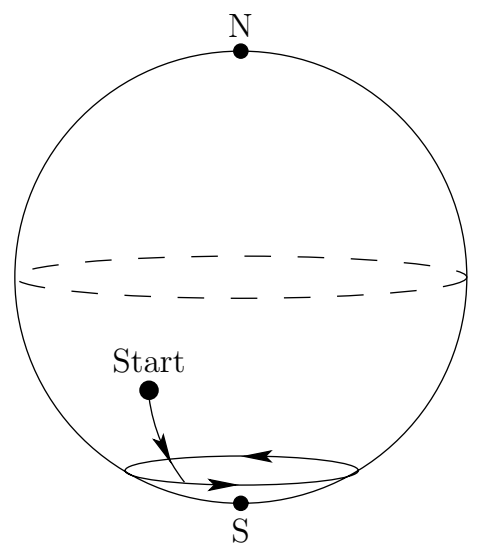

Die Tarnjacke des Abenteurers hat auf jeden Fall die Farbe weiß, egal ob er am Nordpol oder in der Nähe des Südpols startet.

L 16.4 Dem Weihnachtsmann gelingt es stets, einen Startpunkt zu finden, von dem aus er die volle Stadionrunde schafft.

Angenommen, es gäbe keinen solchen Startpunkt. Betrachten wir eine Stelle 1, von der aus der Weihnachtsmann mindestens so viele Stationen erreicht wie von jedem anderen Anfangsort. Nummerieren wir die Stationen in Laufrichtung von 1 bis 17, so gelangt der Weihnachtsmann nach unserer Annahme dabei aber nicht bis zu seinem Startpunkt, sondern nur bis zu einer Station $x$, wobei $x \leq 17$. Nach der Wahl von Station 1 liegt an Station 17 nicht genügend Futter bereit, um zu Station 1 zu gelangen. Ansonsten würde der Weihnachtsmann von Punkt 17 aus alle Stationen erreichen, die er von Station 1 startend erreicht, was unserer Auszeichnung von Punkt 1 widerspricht. An Station 16 und 17 zusammen gibt es nicht so viel Futter, wie für die Schlittenfahrt von Station 16 zu 1 nötig wäre. Der Weihnachtsmann fände sonst an Station 16 so viel Futter, dass er bis zu Station 17 käme und noch 
genug übrig hätte, um von dort aus zu Station 1 zu gelangen. Entsprechend gibt es an den Stationen 15, 16, 17 zusammen nicht ausreichend Futter, um Station 1 anzufahren, und so weiter. Schließlich steht an den Stationen $x+1, x+2, \ldots, 17$ insgesamt weniger Futter zur Verfügung, als unser Weihnachtsschlitten braucht, um die Strecke von Station $x+1$ bis 1 zurückzulegen. Außerdem liegt an den Stationen 1 bis $x$ nicht so viel Futter bereit, wie für die Strecke von Station 1 bis $x+1$ benötigt wird. Insgesamt also gibt es an allen Stationen zusammen nicht ausreichend Futter, um die Runde zurückzulegen. Dies ist ein Widerspruch zur Aufgabenstellung. Also war unsere Annahme falsch, und der Weihnachtsmann kann immer einen Startpunkt derart finden, dass er die ganze Runde durchfahren kann. 


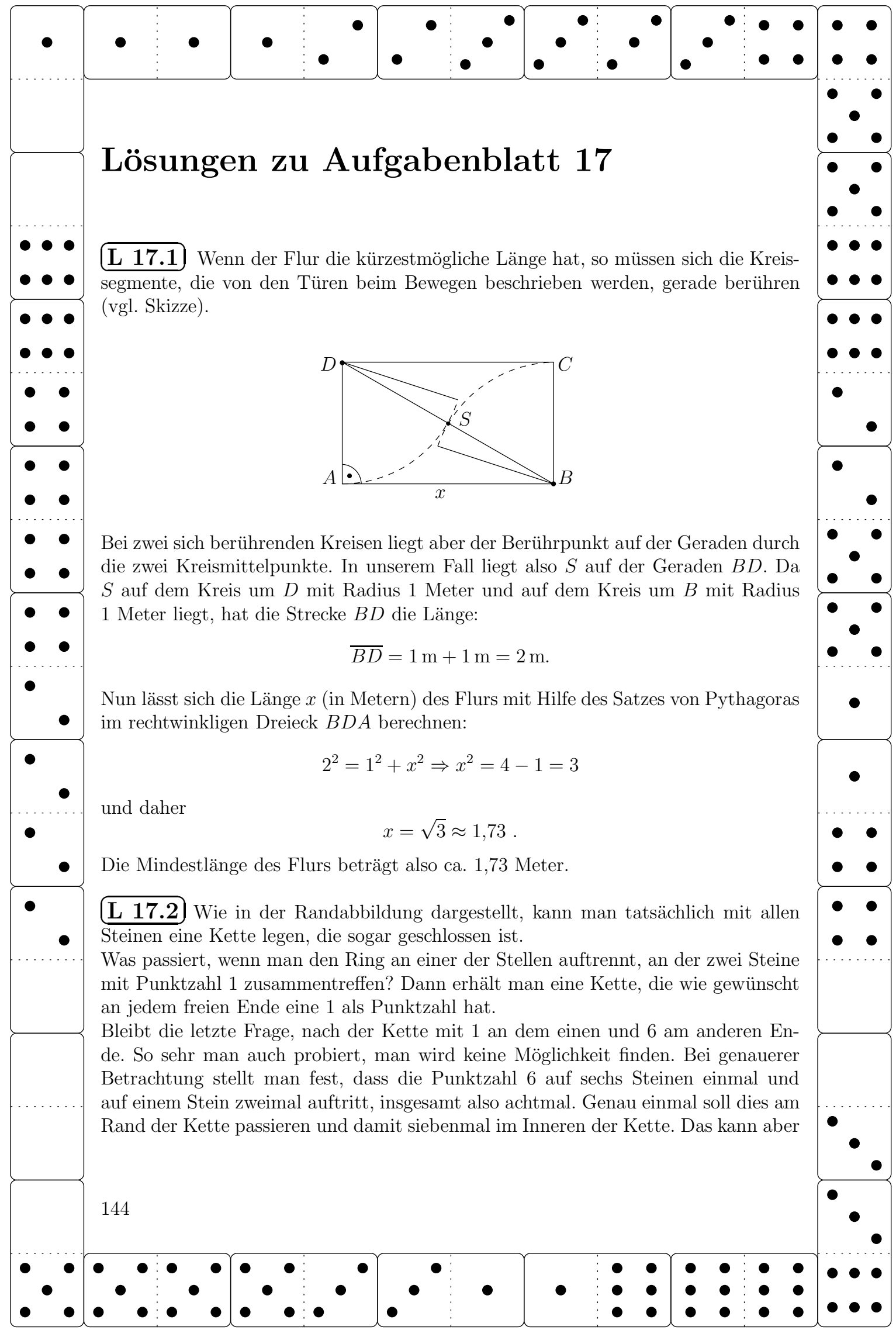


nicht gehen, denn aneinander stoßende Steinhälften müssen immer gleiche Punktzahlen tragen. Damit kann die Punktzahl 6 im Inneren der Kette nur geradzahlig oft auftreten, aber nicht siebenmal. Deshalb kann es keine Kette geben, die an einem Ende die Punktzahl 1 und am anderen Ende die Punktzahl 6 trägt.

L 17.3 Man kann die Figur der Aufgabenstellung gemäß zerlegen.

Die Lösung ist in folgender Zeichnung dargestellt:

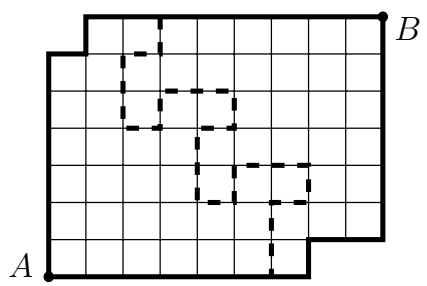

Und natürlich kann man dies auch logisch ableiten und muss sich nicht auf Glück beim Probieren verlassen; dies soll kurz erläutert werden:

Das Kästchen 1 ist in gerader Richtung sieben Kästchen von dem Kästchen bei B entfernt. Es gibt jedoch kein Kästchen, das von A aus sieben Kästchen in gerader Richtung entfernt ist. Daher muss $1 \mathrm{zu}$ A gehören. B muss ein entsprechendes Kästchen besitzen. Wenn der Figurenteil von B aus dem Figurenteil zu A nur aus einer Drehung hervorginge, müsste dies $1^{*}$ sein, was offensichtlich nicht geht. Daraus kann man aber noch nicht schließen, wie einige es getan haben, daß es keine Aufteilung gibt. Denn die Teile können auch aus einer Drehung und einer Spiegelung hervorgehen, und das entsprechende Kästchen ist dann $1^{\prime}$.

\begin{tabular}{c|c|c|c|c|c|c|c|c|c|}
$2^{*}$ & 1 & 2 & $3^{\prime}$ & $3^{\prime}$ & $3^{\prime}$ & $3^{\prime}$ & $3^{\prime}$ & $3^{\prime}$ \\
\hline 3 & 3 & $1^{\prime}$ & $3^{\prime}$ & $3^{\prime}$ & $3^{\prime}$ & $3^{\prime}$ & $3^{\prime}$ & $3^{\prime}$ \\
\hline 3 & 3 & $2^{\prime}$ & 4 & 4 & $4^{\prime}$ & $4^{\prime}$ & $4^{\prime}$ & $4^{\prime}$ \\
\hline 3 & 3 & 4 & 4 & $4^{\prime}$ & $4^{\prime}$ & $4^{\prime}$ & $4^{\prime}$ & $4^{\prime}$ \\
\hline 3 & 3 & 4 & 4 & $4^{\prime}$ & 5 & 5 & $5^{\prime}$ & $5^{\prime}$ \\
\hline 3 & 3 & 4 & 4 & 5 & 5 & $5^{\prime}$ & $5^{\prime}$ & $5^{\prime}$ \\
\hline 3 & 3 & 4 & 4 & 5 & 5 & $5^{\prime}$ & $1^{*}$ \\
\hline
\end{tabular}

Würde nun 2 zu B gehören, wäre 2* das entsprechende zu A gehörende Kästchen. Daher muss auch $2 \mathrm{zu}$ A gehören und daher $2^{\prime}$ zu B. Da es von A aus kein Kästchen oberhalb von 1 gibt, gibt es zu B kein Kästchen links von $1^{\prime}$. Also gehören alle Kästchen 3 zu A und alle Kästchen $3^{\prime}$ zu B.

Da die Kästchen $1^{\prime}, 2^{\prime}$ und $3^{\prime}$ nicht zu A gehören, gehören die Kästchen 4 nicht zu $\mathrm{B}$, also zu A, und die $4^{\prime}$ gehören zu B. Entsprechend erfolgen die Zuordnungen von 5 und $5^{\prime}$ - fertig, wie eine Probe zeigt. 
Lösungen zu Aufgabenblatt 17

L 17.4 Es ist möglich, die fehlenden Ziffern zu rekonstruieren, ohne dass man das Produkt erneut ausrechnen muss.

Hierzu muss man sich etwas zu den Teilern von 42! überlegen und gewisse Teilbarkeitsregeln kennen.

Zuerst wird die Anzahl der Nullen berechnet, auf die die Zahl endet.

Also muss man berechnen, durch welche 10er-Potenz 42! teilbar ist, d. h. wie oft man nacheinander durch 10 ohne Rest teilen kann. Hierzu braucht man die Anzahl der Faktoren 2 und 5 in der Primfaktorzerlegung von 42 !. Es ist $42 !=42 \cdot 41 \cdot \ldots \cdot 2 \cdot 1$ und daher muss man nur die Anzahlen in den einzelnen Faktoren zählen und diese addieren. Jede gerade Zahl enthält einen Faktor 2. Jede durch $4=2 \cdot 2$ teilbare Zahl jedoch sogar zwei Faktoren 2 und so weiter. Anders ausgedrückt, erhält man die Gesamtzahl, wenn man alle geraden Zahlen zählt, für jede durch 4 teilbare Zahl noch einmal 1 dazuzählt, für jede durch 8 teilbare Zahl wiederum 1 dazuzählt und so weiter. Insgesamt sind es von 1 bis 42 genau 21 gerade Zahlen, zehn durch 4 teilbare Zahlen, fünf Zahlen, die durch 8 teilbar sind, zwei durch 16 und eine durch 32 teilbare Zahl. Damit tritt der Faktor 2 in der Primfaktorzerlegung von 42! genau $21+10+5+2+1=39$-mal auf. Entsprechend erhält man acht Zahlen, die durch 5 teilbar sind, und eine, die durch $25=5 \cdot 5$ teilbar ist. 42 ! hat also $8+1=9$-mal die 5 als Primfaktor. Sie endet also auf 9 Nullen, weshalb die letzte verwischte Ziffer eine Null gewesen ist.

Erste Möglichkeit, die anderen beiden Ziffern zu bestimmen:

Hier werden die Teilbarkeitsregeln für die Neun und für die Elf verwendet.

Eine Zahl ist genau dann durch 9 teilbar, wenn ihre Quersumme durch 9 teilbar ist. Eine Zahl ist genau dann durch 11 teilbar, wenn ihre alternierende Quersumme durch 11 teilbar ist, wobei die alternierende Quersumme gebildet wird, indem man von der ersten Ziffer die zweite subtrahiert, dann die dritte Ziffer addiert, die vierte wieder subtrahiert und so weiter. Bezeichnet man nun die verwischten Ziffern mit $a$ und $x$, so ergibt sich für die Quersumme

$$
Q(42 !)=97+a+25+x+60=a+x+182
$$

und für die alternierende Quersumme

$$
A Q(42 !)=1-4+0-\ldots+4-a+1-\ldots-4+x-5+\ldots+4=-a+x-34 .
$$

Da 42! durch 11 teilbar ist und -34 beim Teilen durch 11 den Rest -1 lässt, muss $-a+x$ durch 11 geteilt den Rest 1 lassen. Somit gilt $-a+x=1$. (Wegen $0 \leq a, x \leq 9$ gilt $-9 \leq-a+x \leq 9$.) Damit gilt $x=a+1$.

Da 42! durch 9 teilbar ist und 182 beim Teilen durch 9 den Rest 2 lässt, muss $a+x$ beim Teilen durch 9 den Rest 7 lassen. Wegen $0 \leq a, x \leq 9$ gilt somit $a+x=7$ oder $a+x=16$ und mit Hilfe der ersten Gleichung $a+a+1=7$ oder $a+a+1=16$, was gleichbedeutend ist zu: $a=3$ oder $a=7,5$.

Daher ist $a=3$ und $x=4$.

Es sind somit alle drei verwischten Ziffern bestimmt. 
Zweite Möglichkeit, die anderen beiden Ziffern zu bestimmen:

Eine weitere Teilbarkeitsregel besagt, dass eine Zahl gerade ist, wenn die letzte Ziffer gerade ist, und dass eine Zahl durch 4 teilbar ist, wenn die Zahl, die aus den letzten zwei Ziffern gebildet wird, durch 4 teilbar ist. Dies liegt daran, dass eine Zahl, die auf zwei Nullen endet, durch $100=4 \cdot 25$ teilbar ist. (Z. B. ist $124=100+24$ durch 4 teilbar, weil 100 durch 4 teilbar ist und auch 24.) Daher ist eine Zahl durch $2^{n}$ teilbar, wenn die aus den letzten $n$ Ziffern bestehende Zahl durch $2^{n}$ teilbar ist. Wir haben oben gesehen, dass 42 ! durch $2^{39}$ teilbar ist. Somit ist $\frac{42 !}{10^{9}}$ durch $2^{30}$ teilbar. Wir können somit Informationen über die nächste verwischte Ziffer erhalten, weil die Zahl, die aus den letzten 15 Ziffern von $\frac{42 !}{10^{9}}$ besteht, durch $2^{15}$ teilbar sein muss. (Hier werden sogar die letzten 15 Ziffern und nicht nur die letzten 13 Ziffern betrachtet, weil sich herausstellen wird, dass man sonst die verwischte Ziffer nicht eindeutig bestimmen kann.) Bezeichnen wir die fehlende Ziffer mit $x$, so können wir die Zahl, die aus den letzten 15 Ziffern gebildet wird, schreiben als:

$$
240511569936384+x \cdot 1000000000000=240511569936384+x \cdot 10^{12}
$$

Diese Zahl soll durch $2^{15}$ teilbar sein:

$$
\begin{aligned}
& 240511569936384+x \cdot 10^{12} \\
= & 8 \cdot\left(30063946242048+x \cdot 10^{9} \cdot 5^{3}\right) \\
= & 2^{6} \cdot\left(3757993280256+x \cdot 10^{6} \cdot 5^{6}\right) \\
= & 2^{9} \cdot\left(469749160032+x \cdot 10^{3} \cdot 5^{9}\right) \\
= & 2^{12} \cdot\left(58718645004+x \cdot 5^{12}\right)
\end{aligned}
$$

Da $58718645004+x \cdot 5^{12}$ noch durch 8 teilbar sein soll und 58718645004 durch 4 teilbar ist, muss $x$ durch 4 teilbar sein, d.h. $x=4 \cdot y$ mit $y=0,1$ oder 2 , da $0 \leq x \leq 9$. Damit ist

$$
58718645004+x \cdot 5^{12}=4 \cdot\left(14679661251+y \cdot 5^{12}\right) .
$$

Da jetzt $14679661251+y \cdot 5^{12}$ gerade sein soll, ist somit $y$ ungerade, also $y=1$, und daher $x=4$.

Um die letzte verwischte Ziffer zu bestimmen, benutzt man noch die Teilbarkeitsregel der Neun: Eine Zahl ist genau dann durch 9 teilbar, wenn ihre Quersumme durch 9 teilbar ist. Bezeichnen wir die letzte fehlende Ziffer mit $a$, so ist die Quersumme von 42 !:

$$
Q(42 !)=97+a+89=186+a
$$

Da 186 beim Teilen durch 9 den Rest 6 lässt, muss also $a=3$ gelten. Es gilt daher

$$
42 !=140500611775287989854 \underline{3} 14260624 \underline{4} 511569936384 \underline{0000000000 ~ . ~}
$$

Bemerkung: Es gibt auch noch andere Möglichkeiten, die Ziffern zu bestimmen, wenn man andere Teilbarkeitsregeln benutzt. 


\section{Lösungen zu Aufgabenblatt 18}

L 18.1 Eine sechsstellige natürliche Zahl, die aus den Ziffern 1,2,3,4,5 und 6 gebildet wird, hat die Quersumme $1+2+3+4+5+6=21$. Also ist sie durch 3 teilbar, aber nicht durch 9. Ist aber eine Quadratzahl $n^{2}$ durch 3 teilbar, so ist auch $n$ durch 3 und damit $n^{2}$ sogar durch 9 teilbar. Es gibt folglich unter den betrachteten Zahlen keine Quadratzahl.

L 18.2 Sechsmal muss der Tischler sägen, um den Würfel zu zerlegen!

Der mittlere Würfel muss von allen Nachbarn getrennt werden. Für jede seiner sechs Seiten ist hierfür ein eigener Schnitt nötig. Somit braucht man mindestens sechs Schnitte.

Wir müssen nun noch zeigen, dass sechs Schnitte ausreichen. Zum Beispiel können wir den Würfel entlang der Seitenflächen des inneren Würfels zersägen, wobei wir abgetrennte Teile an ihrem Platz belassen.

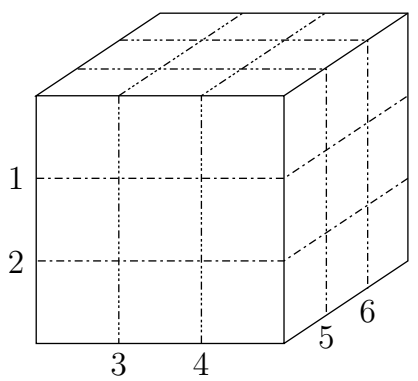

Abbildung 18.1: Zerschneiden des Würfels

L 18.3 Wir bestimmen zunächst die Punkte, die Anke und Ingolfs Ball zur gleichen Zeit erreichen (bei maximalen Geschwindigkeiten). Hierzu betrachten wir die Zeichnung in Abbildung 18.2.

$I$ und $A$ seien die Standorte von Ingolf bzw. Anke. $T$ sei ein Punkt, den Anke und Ingolfs Ball gleichzeitig erreichen, d.h. die Strecke $I T$ ist dreimal so lang wie die Strecke $A T$. Seien weiter $P$ und $Q$ die Punkte auf der Geraden $(I A)$, welche Anke und Ingolfs Ball gleichzeitig erreichen, wobei $P$ zwischen $I$ und $A$ liege. Die Parallele zu $(P T)$ durch $A$ schneide die Gerade $(I T)$ im Punkt $S$ und die Parallele zu (TQ) durch $A$ schneide die Gerade $(I T)$ im Punkt $R$. 


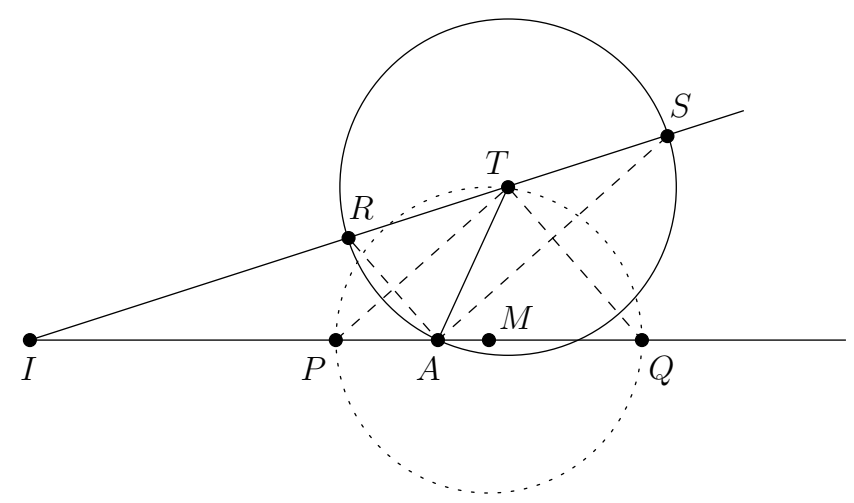

Abbildung 18.2: Berechnung der Punkte, die gleichzeitig erreicht werden

Nach dem ersten Strahlensatz gilt nun:

$$
\frac{\overline{R T}}{\overline{I T}}=\frac{\overline{A Q}}{\overline{I Q}}=\frac{1}{3} \quad \Longrightarrow \overline{R T}=\frac{1}{3} \cdot \overline{I T}=\overline{A T} .
$$

und

$$
\frac{\overline{S T}}{\overline{I T}}=\frac{\overline{A P}}{\overline{I P}}=\frac{1}{3} \quad \Longrightarrow \overline{S T}=\frac{1}{3} \cdot \overline{I T}=\overline{A T} .
$$

$R$ und $S$ liegen also auf dem Kreis um $T$ mit Radius $\overline{A T}$ oder anders betrachtet liegt dann $A$ auf dem Kreis mit Durchmesser $\overline{R S}$. Nach dem Satz des Thales ist also der Winkel SAR ein rechter Winkel.

Da aber $P T$ parallel zu $A S$ und $Q T$ parallel zu $A R$ sind, ist somit auch der Winkel $P T Q$ ein rechter Winkel. $T$ liegt somit auf dem Kreis mit Durchmesser $\overline{P Q}$. Umgekehrt kann man auch leicht zeigen, dass jeder Punkt auf diesem Kreis genau dreimal so weit von $I$ wie von $A$ entfernt ist.

Also bilden die Punkte, die Anke und Ingolfs Ball gleichzeitig erreichen, einen Kreis mit Durchmesser $\overline{P Q}$.

Berechnungen der Strecken:

$$
\begin{array}{ll}
\overline{I A}=\overline{I P}+\overline{P A}=\overline{I P}+\frac{1}{3} \cdot \overline{I P}=\frac{4}{3} \cdot \overline{I P} \quad \Longrightarrow \overline{I P}=\frac{3}{4} \cdot \overline{I A}=15 \mathrm{~m} \\
\overline{I A}=\overline{I Q}-\overline{A Q}=\overline{I Q}-\frac{1}{3} \cdot \overline{I Q}=\frac{2}{3} \cdot \overline{I Q} \quad \Longrightarrow \overline{I Q}=\frac{3}{2} \cdot \overline{I A}=30 \mathrm{~m} \\
\overline{I M}=\frac{1}{2} \cdot(\overline{I Q}+\overline{I P})=22,5 \mathrm{~m} \\
\overline{M P}=\frac{1}{2} \cdot \overline{P Q}=\frac{1}{2} \cdot(\overline{I Q}-\overline{I P})=7,5 \mathrm{~m} .
\end{array}
$$




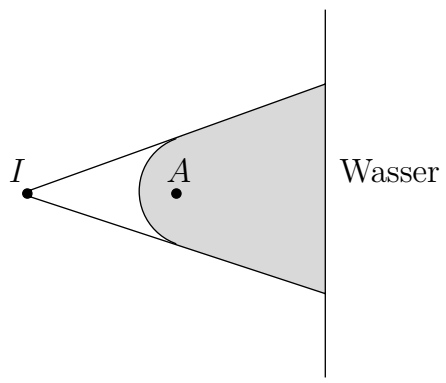

Abbildung 18.3: Der geschützte Bereich

Alle Punkte innerhalb dieses Kreises kann Anke schneller erreichen als der Ball. Aber auch die Punkte, die zusätzlich in der Zeichnung schraffiert sind, sind sichere Standorte, da Anke den bösen Ball auf seinem Weg dorthin, der den Kreis in jedem Fall schneidet, rechtzeitig abfangen kann.

Bemerkung: Sind zwei verschiedene Punkte $A$ und $B$ gegeben, so bilden diejenigen Punkte $P$, für die das Verhältnis der Abstände zu $A$ und $B$ einen konstanten Wert annimmt, einen Kreis, den sogenannten Kreis des Apollonius (Apollonius von Perga, 262-190 v. u. Z.).

L 18.4 Wenn Asterix die Dreierfolge $A A Z$ und Obelix die Dreierfolge $Z A A$ hat, gewinnt Asterix mit der Wahrscheinlichkeit 1/4. Das sieht man am besten wie folgt ein: Wenn zu Anfang zwei $A$ hintereinander geworfen werden (das geschieht offensichtlich mit der Wahrscheinlichkeit 1/4), gewinnt Asterix, nämlich sowie das erste $Z$ fällt. Fängt die Folge aber anders an, ist demnach schon ein $Z$ geworfen worden. Sobald nun zum ersten Mal zwei $A$ hintereinander geworfen werden (was für einen Gewinn von Asterix notwendig wäre), ist vorher schon ein $Z$ geworfen worden, daher gewinnt nun Obelix.

Falls jetzt Obelix die Folge $A Z A$ gewählt hat, sieht die Sache etwas komplizierter aus. Es bezeichne $x$ die Wahrscheinlichkeit, mit der Asterix gewinnt. Wie oben gewinnt Asterix, wenn zu Anfang zwei $A$ geworfen werden (Wahrscheinlichkeit 1/4). Wenn zu Anfang $A Z A$ fällt, gewinnt Obelix. Fällt $A Z Z$ (Wahrscheinlichkeit 1/8), so kann keiner der beiden einen Rest dieser Kombination nutzen (da beide Gewinnkombinationen auf $A$ anfangen) und daher ist die Wahrscheinlichkeit für einen Gewinn wie am Anfang. Gleiches gilt auch schon, wenn gleich zu Anfang ein $Z$ geworfen wird (Wahrscheinlichkeit 1/2). Daher gilt:

$$
\begin{aligned}
x & =\frac{1}{4}+0+\frac{1}{8} x+\frac{1}{2} x \\
\Longleftrightarrow \quad x & =\frac{2}{3} .
\end{aligned}
$$

Hier gewinnt also Asterix mit größerer Wahrscheinlichkeit. 


\section{Lösungen zu Aufgabenblatt 19}

\section{19.1}

a) Können im „Buch der Wahrheit“ zwei oder mehr richtige Aussagen stehen? Das würde dann bedeuten, dass sowohl eine Aussage „In diesem Buch stehen genau $x$ falsche Aussagen“ als auch eine andere Aussage „In diesem Buch stehen genau y falsche Aussagen" gleichzeitig wahr sein müssten. Das ist aber wegen des Wörtchens ,genau“ ein Widerspruch.

Ist andererseits überhaupt keine Aussage im Buch wahr, dann müssten alle 2002 Aussagen falsch sein. Dann wäre aber doch eine Aussage wahr, nämlich die auf Seite 2002, also führt dies ebenfalls zum Widerspruch.

Es verbleibt als letzte und einzige Möglichkeit, dass genau eine Aussage wahr ist, und die anderen 2001 falsch. Dies führt zu keinem Widerspruch: Die wahre Aussage ist die auf Seite 2001, alle anderen 2001 Aussagen sind falsch.

b) Hier kommt es auf das Wörtchen „mindestens“ an. Wenn die Aussage auf einer bestimmten Seite richtig ist (z. B. Seite 10), dann sind die Aussagen auf allen vorhergehenden Seiten ebenfalls korrekt (in diesem Fall dann die Seiten 1 bis 9). Es gibt also in diesem Buch höchstens eine „magische“ Seite: Die Aussagen auf allen Seiten davor und auf der Seite selbst sind richtig, alle Aussagen danach sind falsch. Die Seitennummer dieser magischen Seite entspricht somit der Anzahl der auf diese Seite folgenden Seiten des Buches, diese Seitennummer ist also gerade die Hälfte der Gesamtseitenzahl. Folglich hat die magische Seite die Seitennummer 1001. Genau 1001 Aussagen des Buches sind falsch, dies sind alle Aussagen der folgenden 1001 Seiten. Die Aussagen der ersten 1001 Seiten sind somit alle wahr.

Bemerkung: Es kann korrekt nur von höchstens einer magischen Seite gesprochen werden, weil die Aufgabe für ein Buch mit ungerader Seitenzahl unlösbar ist: Die Aussage auf der mittleren Seite kann weder wahr noch falsch sein.

L 19.2 Zwei Türme bedrohen sich bekanntlich genau dann, wenn sie in einer gemeinsamen Zeile oder einer gemeinsamen Spalte des Schachbrettes stehen.

Um vier Türme ohne gegenseitige Bedrohung zu platzieren, muss man also zunächst vier verschiedene Zeilen und unabhängig davon vier verschiedene Spalten aus den jeweils acht zur Verfügung stehenden auswählen. Hierfür hat man je $\left(\begin{array}{l}8 \\ 4\end{array}\right)=\frac{8 \cdot 7 \cdot 6 \cdot 5}{4 \cdot 3 \cdot 2 \cdot 1}$ Möglichkeiten ${ }^{1}$.

\footnotetext{
${ }^{1}$ Allgemein geben die so genannten Binomialkoeffizienten $\left(\begin{array}{l}n \\ k\end{array}\right)=\frac{n \cdot(n-1) \cdot \ldots \cdot(n-k+1)}{k \cdot(k-1) \cdot \ldots \cdot 1}$ gerade die Anzahl der Möglichkeiten, $k$ Elemente aus einer $n$-elementigen Menge auszuwählen, an. Siehe dazu auch die Erklärungen auf dem Aufgabenblatt 9 zur „Zwergenaufgabe“.
} 
Schließlich darf man in jeder der vier ausgewählten Zeilen die Spalte wählen, in die der Turm soll. Für die erste Zeile hat man vier Spalten zur Verfügung, für die zweite nur noch drei, für die dritte noch zwei und die letzte Spalte steht dann fest. Das sind noch einmal $4 \cdot 3 \cdot 2 \cdot 1$ Möglichkeiten, die Türme in die gewählten Zeilen und Spalten zu stellen.

Insgesamt ergeben sich also

$$
\left(\begin{array}{l}
8 \\
4
\end{array}\right)^{2} \cdot 4 \cdot 3 \cdot 2 \cdot 1=\frac{8^{2} \cdot 7^{2} \cdot 6^{2} \cdot 5^{2}}{4 \cdot 3 \cdot 2 \cdot 1}=117600
$$

Möglichkeiten.

Alternativ kann man auch so zählen: Für den ersten Turm hat man 64 mögliche Felder. Da er genau 15 Felder bedroht (inklusive des eigenen), bleiben für den zweiten Turm noch 49 mögliche Felder. Der zweite Turm bedroht genau 13 weitere Felder, so dass man für den dritten Turm noch 36 Felder zur Verfügung hat und schließlich für den letzten Turm noch 25.

Da es auf die Reihenfolge der Türme nicht ankommt, erhält man jede Stellung hierbei $4 \cdot 3 \cdot 2 \cdot 1 \mathrm{Mal}$, so dass sich auch hier wieder 117600 Möglichkeiten ergeben.

\section{19.3}

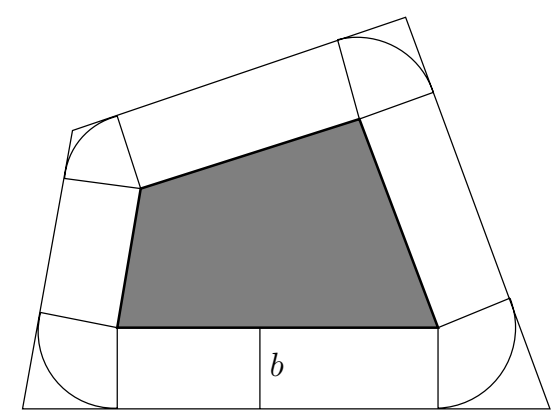

Um die Fläche des Rahmens abzuschätzen, werden zuerst die Senkrechten zu den Polygonseiten durch die Eckpunkte des Polygons gezeichnet (vgl. Skizze). Dadurch wird der Rahmen in Rechtecke und kleine Eckstücke zerlegt. Die Rechtecke überschneiden sich nicht, weil das Polygon konvex ist; sie haben alle eine Breite von $b$ und die Summe der Längen der Rechtecke ist gerade der Umfang $U$ des Polygons, weshalb sie zusammen einen Flächeninhalt von $b \cdot U$ haben. Nun sind noch die kleinen Eckstücke zu betrachten. In den Rahmen lassen sich Kreisbögen mit Radius $b$ einbeschreiben. Die Winkel der entstehenden Kreissegmente addieren sich gerade zu $360^{\circ}$, weshalb die Summe der Flächen der Eckstücke größer ist als eine Kreisfläche mit Radius $b$, d. h. größer als $\pi \cdot b^{2}$.

Die Rahmenfläche ist also größer als $b \cdot U+\pi \cdot b^{2}=b(U+\pi b)$. 
L 19.4 Zu a): Das Argument ist deswegen nicht anwendbar, weil die Bedingungen, unter denen mit dem Werfen der Münze aufgehört wird, mit dem Auftreten der Tripel zusammenhängen. Würden immer gleich lange Ketten geworfen werden (oder auch mit einer jedes Mal von den Würfen unabhängig per Zufall bestimmten Länge) und dann gezählt, wie oft welche Kombination gekommen ist, hätte keiner einen Vorteil. Bei diesem Spiel ist es aber von Bedeutung, dass es z. B. bei der Paarung $A A Z$ gegen $Z A A$ sehr wahrscheinlich ist, dass vor dem Auftreten eines Tripels $A A Z$ ein Tripel $Z A A$ kam. Die einzige Ausnahme (und damit einzige Chance für Asterix) ist der Fall, dass gleich zu Anfang mindestens zwei $A$ geworfen werden.

„Ja, aber, ... heißt das dann nicht, dass irgendwie ZAA im Mittel früher geworfen wird als $A A Z$ ? " Das stünde in der Tat im Widerspruch zu der Tatsache, dass ohne wurfabhängige Abbruchbedingung alle Tripel gleich oft vorkommen. Aber die vermeintliche Folgerung ist auch direkter zu entkräften: Zwar kommen nach Werfen des ersten $Z$ die Tripel $Z A A$ und $A A Z$ immer paarweise vor, wobei $Z A A$ vorangeht. Aber es kann ja gleich zu Anfang $A \ldots A Z$ geworfen worden sein. Dann kommt immer $A A Z$ zuerst. Dies geschieht zwar nur mit der Wahrscheinlichkeit 1/4. Aber wenn ein $A A Z$ kommt, kann das nächste $Z A A$ frühestens zwei Stellen später vorkommen, während nach einem $Z A A$ das nächste $A A Z$ direkt folgen kann (und sozusagen „zwingend“, nämlich nach Ende der $A$-Kette erscheint). Dadurch wird der scheinbare Vorteil von $Z A A$ wieder ausgeglichen.

$\mathrm{Zu}$ b): So einfach kann man leider auch nicht schließen. (Der Mathematiker sagt: Die Relation „siegt gegen“ ist nicht transitiv.) In der Tat sind $Z A A$ und $A Z A$ gegeneinander gleichwertig:

Sei $x_{A}$ die Gewinnwahrscheinlichkeit für $A Z A$ in dem Fall, dass als Erstes ein $A$ geworfen wurde; $x_{Z}$ diejenige für den Fall, dass als Erstes ein $Z$ geworfen wurde.

Sei zunächst ein $A$ geworfen worden. Fällt ein weiteres $A$, ist das erste $A$ für beide Spieler wertlos und die Situation so, als ob nur ein $A$ liegen würde. Fällt stattdessen $Z A$, so hat $A Z A$ gewonnen. Fällt $Z Z$, so ist die Situation so, als ob nur ein $Z$ geworfen worden wäre. Also gilt:

$$
x_{A}=1 / 2 x_{A}+1 / 4+1 / 4 x_{Z} .
$$

Ist am Anfang ein $Z$ gefallen, ist Folgendes zu unterscheiden: Fällt $A A$, gewinnt $Z A A$. Fällt $A Z A$, gewinnt offensichtlich $A Z A$; fällt $A Z Z$, ist die Situation wie bei nur einem geworfenen $Z$, ebenso bei einem weiteren $Z$. Das ergibt

$$
\begin{aligned}
x_{Z} & =1 / 4 \cdot 0+1 / 8+1 / 8 x_{Z}+1 / 2 x_{Z} \\
\Leftrightarrow x_{Z} & =1 / 3 .
\end{aligned}
$$

Weiterhin folgt daraus

$$
\begin{aligned}
x_{A} & =2 \cdot(1 / 4+1 / 4 \cdot 1 / 3)=2 / 3 \text { und } \\
x & =1 / 2 x_{A}+1 / 2 x_{Z}=1 / 2
\end{aligned}
$$


Lösungen zu Aufgabenblatt 19

für die Wahrscheinlichkeit, dass $A Z A$ gewinnt. Mithin gewinnt $Z A A$ mit der gleichen Wahrscheinlichkeit.

Bemerkung: Das Phänomen der Intransitivität ist übrigens weniger verwunderlich, als es auf den ersten Blick erscheinen mag: Wenn im Sport meistens $A$ gegen $B$ und $B$ gegen $C$ gewinnt, heißt das ja noch nicht, daß meistens auch $A$ gegen $C$ gewinnt. Die Taktik von $A$ kann ja so sein, daß sie $C$ nicht beeindruckt. Ein konkretes Beispiel nannte ein Teilnehmer: Beim Spiel „Schere, Stein, Papier" wird „im Kreis" gewonnen.

Auch beim Spiel von Asterix und Obelix gibt es so eine Folge (übrigens nur genau diese eine): Es gewinnen

- $Z A A$ gegen $A A Z$ mit $3 / 4$ zu $1 / 4$,

- $A A Z$ gegen $A Z Z$ mit $2 / 3$ zu $1 / 3$,

- $A Z Z$ gegen $Z Z A$ mit $3 / 4$ zu $1 / 4$,

- $Z Z A$ gegen $Z A A$ mit $2 / 3$ zu $1 / 3$.

Das Spiel bietet noch weiteren Stoff für interessante Entdeckungen. Wenn acht Leute mit allen acht verschiedenen Tripeln mitspielen, ist es wieder ein faires Spiel, denn es wird jedesmal nach drei Würfen geendet. Was passiert jedoch „,auf dem Weg dahin“, d. h. wenn man nach und nach die Spieleranzahl erhöht? Müssen sich die Verhältnisse immer weiter angleichen? Das ist nicht der Fall. Ein Beispiel: $Z A Z$ gewinnt gegen $A A A$ mit $4 / 7 \mathrm{zu} 3 / 7$. Wenn nun zu dritt mit $Z A A$ gespielt wird (das gegen $Z A Z$ neutral spielt und gegen $A A A$ mit $7 / 8 \mathrm{zu} 1 / 8$ gewinnt), so ist das Gewinnverhältnis: $Z A Z: A A A: Z A A=7 / 16: 1 / 8: 7 / 16$. Im direkten Vergleich zwischen $Z A Z$ und $A A A$ steht es jetzt also haushohe $7 / 16$ zu $2 / 16$ ! 


\section{Lösungen zu Aufgabenblatt 20}

L 20.1 Im Folgenden eine (vermutlich die schnellste) mögliche Lösung (wahrscheinlich ist es hilfreich, wenn du die Anweisungen beim Lesen auf einem Modell ausführst):

- Die Lok schiebt Wagen 1 bis zur Brücke und zieht dabei Wagen 2 hinter sich her.

- Wagen 2 wird aufs Abstellgleis geschoben.

- Die Lok kehrt zu Wagen 1 zurück und zieht ihn anschließend bis zu seinem Ausgangspunkt.

- Die Lok fährt zum ersten Mal unter der Brücke durch und koppelt Wagen 2 an.

- Die Lok zieht zuerst Wagen 2 zurück aufs Hauptgleis und schiebt ihn dann bis zu Wagen 1.

- Nachdem Wagen 1 angekoppelt ist, werden beide Wagen gezogen und anschließend aufs Abstellgleis - Wagen 1 voran - geschoben.

- Wagen 1 wird wieder abgekoppelt und Wagen 2 bis zur Brücke gezogen.

- Anschließend fährt die Lok zum zweiten und letzten Mal unter der Brücke durch zur anderen Seite von Wagen 2.

- Die Lok fährt wieder aufs Abstellgleis, koppelt Wagen 1 an und zieht ihn bis zu Wagen 2.

- Zuletzt fährt die Lok, Wagen 1 voranschiebend und Wagen 2 hinterherziehend, zu ihrem Ausgangspunkt.

Nun sind Wagen 1 und Wagen 2 vertauscht.

L 20.2 Eine Lösung der Aufgabe ist:

$$
\frac{6}{1-\frac{5}{7}}
$$

Es ist uns nicht bekannt, ob es noch weitere Lösungen gibt. 
L 20.3 Egal bei welcher Zahl man beginnt, man erreicht im Laufe der Prozedur immer die Zahl 6174 und bleibt auch bei ihr, da $7641-1467=6174$ gilt.

Es gibt (noch) keine Methode, dies zu beweisen, ohne einige Zahlen durchzurechnen, aber es ist zumindest nicht nötig, alle 8991 vierstelligen Zahlen, die nicht aus lauter gleichen Ziffern bestehen, nachzuprüfen. Durch einige vorbereitende Überlegungen erhält man, dass es sogar ausreicht, 13 Zahlen zu überprüfen. Genauere Angaben inklusive Beweis kann man zum Beispiel unter http://www.MathePrisma.uniwuppertal.de/Module/6174/index.htm nachlesen.

L 20.4 Ausgehend von dem umrahmten Rechteck in der Mitte des Musters werden die Felder spiralförmig gegen den Uhrzeigersinn durchnummeriert. Soll heißen, das umrahmte Rechteck bekommt die Nummer 1, das rechts daneben die 2. Über der 2 kommt die 3, links von der 3 die 4 und so weiter.

Dann werden die Stellen schwarz gefärbt, an denen eine Primzahl steht. So erhält man dieses Muster.

Die weiße Diagonale vom Mittelpunkt nach rechts unten ergibt sich zum Beispiel dadurch, dass die Zahlen auf der Diagonalen alles Quadratzahlen sind und somit keine Primzahlen sein können. Da diese Quadratzahlen alle ungerade sind, liegen also rechts und links davon gerade Zahlen - außer der 2 auch keine Primzahlen -, weshalb auch diese Felder weiß bleiben. Und schon hat man eine breitere weiße Diagonale.

Ähnlich verhält es sich bei den anderen Streifen. 


\section{Lösungen zu Aufgabenblatt 21}

L 21.1 Es sei $k$ die Länge der gesuchten arithmetischen Folge. Dann kann man beispielsweise die Folge

$$
\frac{k}{k !}, \frac{k-1}{k !}, \ldots, \frac{2}{k !}, \frac{1}{k !}
$$

auswählen. Dabei ist $k$ ! (sprich „,k Fakultät“) die mathematische Schreibweise für das Produkt $1 \cdot 2 \cdot 3 \cdot \ldots \cdot k$.

Jeden dieser Brüche kann man durch den Zähler kürzen, wonach im Zähler eine 1 stehen bleibt. Da die Differenz zweier aufeinander folgender Glieder stets $\frac{1}{k !}$ ist, hat man somit eine gesuchte Folge mit $k$ Gliedern gefunden.

L 21.2 Die einzelnen Eckpunkte seien wie in nachstehender Zeichnung bezeichnet. Außerdem werden zusätzlich noch die Strecken $P B$ und $P C$ eingezeichnet. Anhand der neuen Zeichnung kann man vielleicht schon eine Vermutung aufstellen, und zwar scheinen die Dreiecke $A B P$ und $C P Q$ kongruent zu sein, weshalb das gesuchte Reststück den gleichen Flächeninhalt hätte wie das Dreieck $P B C$. Des Weiteren scheint das Dreieck $P B C$ zu dem Dreieck $A P D$ kongruent zu sein. Demnach wäre der Flächeninhalt des Reststücks genauso groß wie der Flächeninhalt des Dreiecks $A P D$. Andererseits sind nach Voraussetzung die Dreiecke $P Q D$ und $Q C D$ ebenfalls kongruent zu dem Dreieck $A P D$. Das Quadrat wäre somit in vier flächengleiche Stücke aufgeteilt. Daher betrüge der Flächeninhalt des Reststücks genau ein Viertel der Quadratfläche, also $\frac{a^{2}}{4}$.

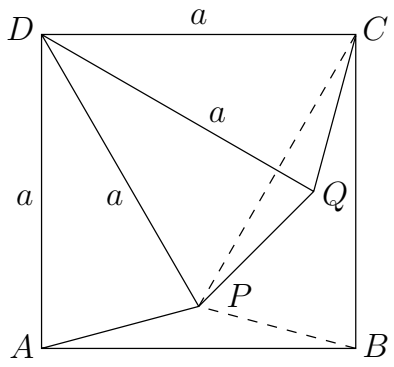

Es wird nun gezeigt, dass sowohl die Dreiecke $A B P$ und $C P Q$ als auch die Dreiecke $A P D$ und $P B C$ tatsächlich kongruent sind, womit der Flächeninhalt des Reststücks $\frac{a^{2}}{4}$ beträgt.

Nach Voraussetzung sind die Dreiecke $A P D, P Q D$ und $Q C D$ kongruent, weshalb die Winkel $\angle A D P, \angle P D Q$ und $\angle Q D C$ gleich und somit je $30^{\circ}$ groß sind, da sie zusammen den rechten Winkel $\angle A D C$ bilden. 
Das Dreieck $P C D$ ist gleichschenklig mit Scheitel $D$ und Scheitelwinkel $\angle P D C=$ $\angle P D Q+\angle Q D C=60^{\circ}$. Damit gilt für die Basiswinkel: $\angle C P D=\angle D C P=$ $\frac{1}{2} \cdot\left(180^{\circ}-60^{\circ}\right)=60^{\circ}$.

Das Dreieck $P C D$ ist also sogar gleichseitig. Daher ist die Länge der Strecke $C P$ gerade $a$ und der Winkel $\angle P C B=90^{\circ}-60^{\circ}=30^{\circ}$. Nach dem Kongruenzsatz sws sind also die Dreiecke $A P D$ und $P B C$ kongruent. Weiter erhält man, dass $\overline{P B}=\overline{A P}$ ist. Also ist $\overline{A P}=\overline{C Q}, \overline{P B}=\overline{Q P}$ und $\overline{A B}=\overline{C P}$. Somit sind auch die Dreiecke $A B P$ und $C P Q$ kongruent.

L 21.3 Die Anzahl der Eisenbahnnetze kann man mit einer Fallunterscheidung über die Zahl $N$ der maximal in einer der Städte endenden Eisenbahnstrecken bestimmen. Da es insgesamt nur vier Strecken geben soll, ist $N \leq 4$. Die vier Bahnstrecken haben insgesamt acht Endpunkte, die sich auf die fünf Städte verteilen. Daher gibt es (nach Schubfachprinzip) auch eine Stadt, in der mindestens zwei Strecken enden. Folglich ist $N \geq 2$. Wir müssen somit die Fälle $N=2, N=3$ und $N=4$ untersuchen

1. Fall: $\mathbf{N}=4$. In diesem Fall gibt es eine Stadt $A$, die direkt mit allen vier anderen Städten verbunden ist. Das Netz hat also folgende Form:

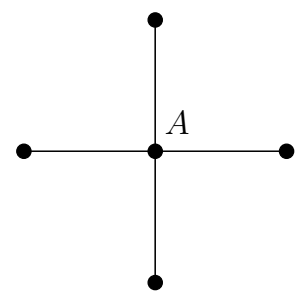

Für die Wahl von $A$ hat man hierbei fünf Möglichkeiten. Es ergeben sich demnach fünf verschiedene solcher Eisenbahnnetze.

2. Fall: $\mathbf{N}=\mathbf{3}$. Keine Stadt hat in diesem Fall vier direkte Nachbarn, aber eine Stadt $A$ hat genau drei Städte, die direkt mit ihr verbunden sind. Die fünfte noch verbleibende Stadt muss dann direkt mit einer dieser drei Städte (sie sei $B$ genannt) verbunden sein. Das Eisenbahnnetz hat dann die folgende Form: 
Hierbei kann man zunächst $A$ unter den fünf Städten beliebig wählen. Danach bleiben für $B$ noch vier Möglichkeiten. Schließlich kann man unter den übrigen drei Städten noch beliebig diejenige bestimmen, die (außer $A$ ) noch direkt mit $B$ verbunden sein soll. Zusammen ergibt dies in diesem Fall $5 \cdot 4 \cdot 3=60$ Netze der abgebildeten Form.

3. Fall: $\mathbf{N}=\mathbf{2}$. In diesem Fall bilden die fünf Städte eine Kette wie in der folgenden Abbildung:

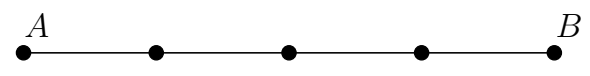

Für die erste Stadt $A$ hat man dabei wieder fünf Möglichkeiten, für die zweite Stadt noch vier usw. bis zur letzten Stadt $B$, für die es nur noch eine Möglichkeit gibt. In dieser Zählung hat man aber jede mögliche Kette genau zweimal gezählt, und zwar einmal von $A$ nach $B$ und einmal von $B$ nach $A$. Daher ergeben sich in diesem Fall genau $\frac{1}{2} \cdot(5 \cdot 4 \cdot 3 \cdot 2 \cdot 1)=60$ mögliche Eisenbahnnetze.

Zusammengenommen erhält man also $5+60+60=125$ verschiedene Eisenbahnnetze.

L 21.4 Zugegeben - dieses Grill-,,Problem“ hört sich zunächst weit hergeholt an. "Auf solche Ideen können nur Mathematiker kommen", hat vielleicht der eine oder andere gedacht, „für die Praxis ist das völlig irrelevant."

Doch damit liegt man falsch: Natürlich genießen auch die meisten Mathematiker ihren Grillabend lieber in Ruhe. Aber solche so genannten Planungsprobleme spielen in der Informatik und in der Industrie eine große Rolle. Wenn man bestimmte Arbeitsabläufe vorgegeben hat, dann möchte man diese in möglichst kurzer Zeit absolvieren, andererseits hat man aber auch nur eine begrenzte Anzahl von Maschinen zur Verfügung. Dasselbe Problem tritt auf, wenn man mehrere Computer zur gemeinsamen Lösung einer Aufgabe verwenden will, auch hier müssen die einzelnen Arbeitsschritte optimal verteilt werden, damit alle Computer möglichst immer ausgelastet sind und nicht ständig auf das Ergebnis einer anderen Rechnung warten müssen.

Für so ein allgemeines Problem eine Lösung zu finden, ist schwierig - so schwierig, dass es länger dauern kann, die optimale Lösung zu finden, als der Vorgang selbst dann überhaupt dauert. Das gilt ja auch für unser Grillproblem: Wahrscheinlich habt ihr zum Finden eurer Lösung und zum Aufschreiben mindestens genauso lange gebraucht wie die Zeit, die ihr letztlich als minimale Grillzeit ermittelt habt.

Wir wollen deshalb einen anderen Weg gehen und fragen zunächst:

Wie viel Zeit brauchen wir eigentlich mindestens?

Damit können wir dann einschätzen, wie gut unsere Ergebnisse sind. Es kann nicht kürzer dauern als die ermittelte Zeit, vermutlich gibt es überhaupt keine Lösung, die so schnell ist. Aber je näher wir an diese Zeit herankommen, desto besser. 
Lösungen zu Aufgabenblatt 21

Jedes Steak muss von beiden Seiten jeweils zweieinhalb Minuten gegrillt werden. Das ergibt bei drei Steaks insgesamt

$$
2 \cdot 2,5 \mathrm{~min} \cdot 3=15 \mathrm{~min} .
$$

Da nur zwei Steaks auf den Grill passen, brauchen wir also mindestens 7,5 Minuten. Unabhängig davon, wie auch immer der genaue Ablauf sein wird - schneller können wir es auf keinen Fall schaffen. Und wir können diese so genannte untere Schranke noch größer machen: Wenn ein rohes Steak auf den Grill gelegt oder ein fertiges heruntergenommen wird, kann gleichzeitig nur ein weiteres Steak auf dem Grill liegen. Das gleiche gilt, wenn wir ein Steak wenden. Die Transportvorgänge dauern also insgesamt mindestens

$$
3 \cdot 15 \mathrm{~s} \cdot 3=135 \mathrm{~s}=2 \min 15 \mathrm{~s} .
$$

Wieder müssen wir diese Zeit durch 2 teilen, da ja jeweils nur ein Grillplatz blockiert wird. In der Summe erhalten wir also eine Mindestzeit von

$$
(15 \min +2 \min 15 \mathrm{~s}) / 2=8 \min 37,5 \mathrm{~s} .
$$

Wichtig ist: Es muss keine tatsächliche Abfolge geben, die diese Zeit erreicht. Das wahre Optimum kann also größer sein, aber auf keinen Fall kleiner.

Jetzt können wir einfach probieren, eine Lösung zu finden, die möglichst nahe an die Mindestzeit herankommt. Wir wissen dann zwar nicht, ob diese wirklich das Optimum ist, aber wir wissen zumindest, wie weit weg es höchstens sein kann.

Zwei sehr gute Lösungen sind in der Abbildung auf der nächsten Seite dargestellt. Die obere, von Jonas ebenso wie von Hartwig, braucht 10 Minuten und 15 Sekunden, die mittlere, von Sabrina, benötigt sogar nur 10 Minuten. Man kann zeigen (und das ist dann die Aufgabe der Mathematiker), dass diese Lösung tatsächlich die beste ist, sofern man alle Seiten immer durchgehend grillt. Man sieht, dass der Grill immer voll ausgelastet ist, lediglich am Ende, wenn das dritte Steak bestrichen wird, scheinen noch Reserven zu liegen.

Mit einem „Trick" kann man diese Reserve noch vermeiden: Man grillt die eine Seite von Steak 1 zunächst nur halb, dann braucht man insgesamt nur 9 Minuten und 30 Sekunden. Das ist schon sehr nahe an der unteren Schranke von 8 Minuten und 37,5 Sekunden. Man kann sogar zeigen, dass es nicht besser geht, indem man durch weitere Überlegungen wie oben die untere Schranke auf 9 Minuten 30 Sekunden vergrößert. 
Steak auf Grill legen

Steak wenden

Steak vom Grill nehmen
Steak wird gegrillt

Steak wird bestrichen

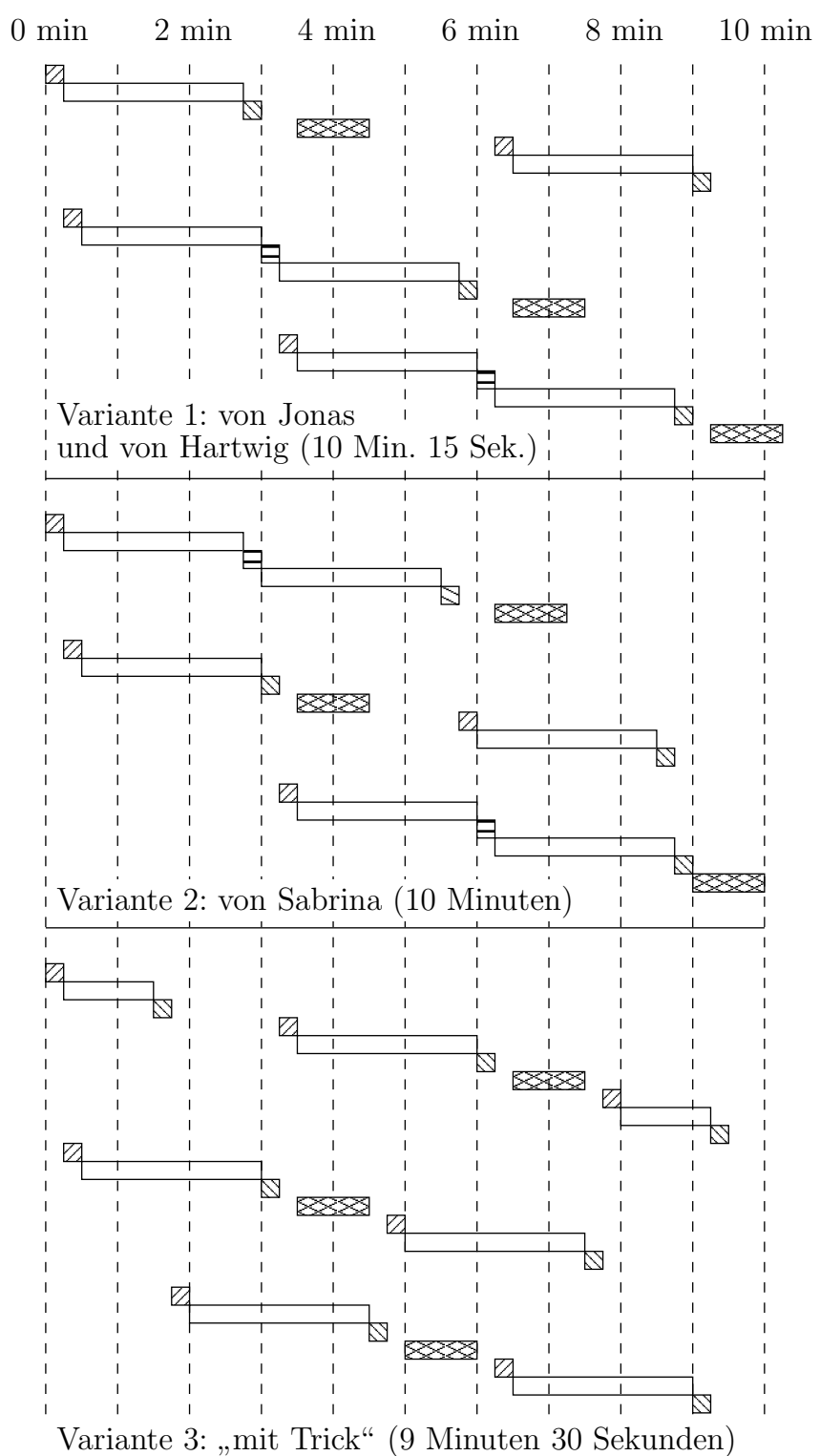




\section{Lösungen zu Aufgabenblatt 22}

L 22.1 Wir beantworten zuerst die Zusatzfrage c), wonach sich die anderen Antworten leicht geben lassen.

Gehen wir davon aus, Leonard habe schon $n-1$ Linien $(n \in \mathbb{N})$ wie in der Aufgabe angegeben gezeichnet. Nun wird gezählt, wie viele Flächenstücke hinzukommen, wenn Leonard die $n$-te Linie zeichnet. Angefangen beim Blattrand beginnt er, die erste Fläche in zwei Teile zu zerlegen. Sobald er auf eine andere Linie trifft, hat er die erste Fläche zerlegt und beginnt eine neue zu zerlegen. Nach Voraussetzung trifft er auf $n-1$ Linien, und zwar jede an einer anderen Stelle, d. h. er hat insgesamt $n-1+1=n$ Flächenstücke zerlegt.

Wenn Leonard also die $n$-te Linie gezeichnet hat, hat sich die Zahl der Flächenstücke um $n$ erhöht.

Wir können also eine rekursive Formel für die Anzahl $A(n)$ der Flächenstücke bei $n$ Linien angeben:

$$
A(n)=A(n-1)+n
$$

Außerdem gilt: $A(0)=1 \quad$ (wenn keine Linie eingezeichnet ist, haben wir eine große Fläche.)

Daraus erhält man:

$$
\begin{aligned}
A(n)= & A(n-1)+n=A(n-2)+(n-1)+n=\ldots \\
= & A(0)+1+2+\ldots+(n-1)+n \\
& \quad \text { und mit der Summenformel } 1+\ldots+n=\frac{n(n+1)}{2} \text { ergibt sich } \\
A(n)= & A(0)+\frac{n(n+1)}{2}=1+\frac{n(n+1)}{2} .
\end{aligned}
$$

Da an keiner Stelle die genaue Lage der Linien benutzt wurde, hängt die Anzahl der Flächenstücke nur von der Anzahl der Linien ab, weshalb die Frage b) mit „Ja“ zu beantworten ist.

Probiert man für $n$ die Zahlen $1,2,3, \ldots$ durch, so erhält man schließlich wegen $1+\frac{6 \cdot 7}{2}=22$, dass Leonard 6 Linien gezeichnet hat. 
L 22.2 Lenas Glückszahl hat eine schöne Eigenschaft. Quadrieren der Zahl

$$
3=\sqrt{x+\sqrt{x+\sqrt{x+\ldots}}}
$$

ergibt die Gleichung

$$
\begin{aligned}
9 & =x+\sqrt{x+\sqrt{x+\sqrt{x+\ldots}}} \\
& =x+3
\end{aligned}
$$

Also ist $x=9-3=6$.

Bemerkung: Wenn es nicht vorausgesetzt wäre, dass mindestens eine Zahl existiert, die die Gleichung erfüllt, so müsste diese Existenz noch gezeigt werden.

L 22.3 Je nach Gestalt des Feldes wird entweder der erste oder der dritte Sohn bevorteilt und der andere benachteiligt. Der zweite und der vierte Sohn bekommen auf jeden Fall je ein Viertel der Gesamtfläche.

Betrachten wir getrennt die Dreiecke $A B D$ und $B C D$.

Im Dreieck $A B D$ sind $M_{a}$ und $M$ nach Aufgabenstellung die Seitenmitten der Seiten $A B$ bzw. $B D$, d. h. bei zentrischer Streckung um $B$ mit Faktor $\frac{1}{2}$ geht das Dreieck $A B D$ in das Dreieck $M_{a} B M$ über. Die Fläche des Dreiecks $M_{a} B M$ ist also gerade $\left(\frac{1}{2}\right)^{2}=\frac{1}{4}$-mal so groß wie die Fläche des Dreiecks $A B D$. Entsprechend geht bei zentrischer Streckung um $D$ mit Faktor $\frac{1}{2}$ das Dreieck $A B D$ in das Dreieck $M_{d} M D$ über, weshalb auch dessen Fläche gerade $\frac{1}{4}$-mal so groß ist wie die des Dreiecks $A B D$.

Die gleiche Überlegung im Dreieck $B C D$ liefert, dass die Flächen der Dreiecke $B M_{b} M$ und $M M_{c} D \frac{1}{4}$-mal so groß wie die des Dreiecks $B C D$ sind.

Daraus erhält man nun folgende Flächengrößen: Der erste Sohn bekommt die Hälfte der Fläche des Dreiecks $A B D$ (zwei Viertel der Dreiecksfläche fehlen) und der dritte Sohn bekommt die Hälfte der Fläche des Dreiecks $B C D$. Für die anderen beiden Söhne bleibt je ein Viertel des Dreiecks $A B D$ und ein Viertel des Dreiecks $B C D$, also gerade ein Viertel der gesamten Fläche.

Somit wird der erste Sohn bevorteilt, wenn das Dreieck $A B D$ eine größere Fläche hat als das Dreieck $B C D$, und der dritte Sohn wird bevorteilt, wenn die Fläche des Dreiecks $A B D$ kleiner ist. Nur wenn beide Flächen gleich sind, ist die Aufteilung gerecht.

Bemerkung: Ist $P$ der Schnittpunkt der Diagonalen $A C$ und $B D$ des Vierecks $A B C D$, so treten die verschiedenen Fälle ein, wenn $\overline{A P}>\overline{P C}$ bzw. $\overline{A P}<\overline{P C}$ bzw. $\overline{A P}=\overline{P C}$ gilt. 
Lösungen zu Aufgabenblatt 22

\section{22.4}

a) Auf Monikas erstem Blatt (dies sei das mit den Anfangszahlen) gibt es eine größte Zahl $n$ (evtl. in mehrfacher Ausführung). Alle Differenzen, die mit ihr gebildet werden, werden mit natürlichen Zahlen gebildet, die größer als null sind. Daher sind diese Differenzen und damit offensichtlich auch alle anderen gebildeten Differenzen kleiner oder gleich $n-1$. Dieselbe Argumentation gilt natürlich weiter für alle anderen Blätter, womit folgt, dass auf dem $k$-ten Blatt alle Zahlen kleiner oder gleich $n-(k-1)$ sind. Da keine Zahlen kleiner gleich 0 vorkommen können, ist spätestens mit dem $(n+1)$-ten Blatt Schluss.

b) Nein, es kann nicht vorkommen, dass Monika am Ende genau eine Zahl auf einem Blatt stehen hat. Zum Beweis sei angenommen, dies sei doch der Fall. Dann müssen auf dem vorletzten Blatt genau zwei, und zwar voneinander verschiedene Zahlen gestanden haben. (Denn wenn die Zahlen alle gleich gewesen wären, wäre keine Differenz ungleich null gewesen, so dass nichts notiert worden wäre; wären es mehr als zwei Zahlen gewesen, die nicht alle gleich sind, so wären darin also mindestens drei Zahlen $a, b, c$ mit $a \neq b \neq c$ (evtl. $a=c$ ) enthalten. Diese hätten jedoch mindestens die beiden Zahlen $|a-b|$ und $|b-c|$ für den letzten Zettel geliefert.)

Um zwei verschiedene Zahlen zu erhalten, müssen auf dem drittletzten Zettel mindestens drei verschiedene Zahlenwerte gestanden haben, $a>b>c$ Diese bilden jedoch drei von null verschiedene Differenzen, $a-b, a-c$ und $b-c$, was zum Widerspruch führt. Daher ist die Annahme zu Beginn falsch, der genannte Fall kann also nicht vorkommen.

Für die richtige Lösung ist wichtig, dass gleiche Differenzen, wenn sie mehrfach auftreten, auch wie angegeben mehrfach notiert werden. Andernfalls wird immer das Ende mit einer verbleibenden Zahl eintreten, wie man sich klarmachen kann. 


\section{Lösungen zu Aufgabenblatt 23}

L 23.1 Nehmen wir an, die Gäste kennen jeweils verschiedene Anzahlen von Gästen. Da es 97 Gäste sind, kann ein Gast keinen, einen, zwei, .. oder 96 Gäste kennen. Dies sind gerade 97 Möglichkeiten. Somit muss jede dieser 97 Möglichkeiten (genau einmal) auftreten. Es gibt also einen Gast, der keinen anderen Gast kennt, und einen Gast, der 96 Gäste, d.h. alle anderen Gäste kennt. Dies ist offensichtlich nicht möglich, da diese zwei Gäste sich entweder kennen, dann kennt aber der erste Gast einen weiteren, oder sich nicht kennen, dann kennt aber der zweite Gast nicht alle anderen Gäste.

Unsere Annahme muss also falsch sein und daher gibt es zwei Gäste, die die gleiche Zahl von Gästen kennen.

L 23.2 Alle natürlichen Zahlen haben diese hübsche Eigenschaft.

Betrachten wir eine beliebige natürliche Zahl $m$. Dann liegt $m$ zwischen den Quadratzahlen $n^{2}$ und $(n+1)^{2}$, das heißt, $n^{2} \leq m<(n+1)^{2}$. Also kann man die gewünschte Zahl wie folgt berechnen:

$$
\begin{aligned}
& m-\left(m-n^{2}\right)\left((n+1)^{2}-m\right) \\
& =m-\left(m n^{2}+2 m n+m-m^{2}-n^{4}-2 n^{3}-n^{2}+m n^{2}\right) \\
& =\left(-2 m n^{2}-2 m n\right)+m^{2}+\left(n^{4}+2 n^{3}+n^{2}\right) \\
& =-2 m\left(n^{2}+n\right)+m^{2}+\left(n^{2}+n\right)^{2} \\
& =\left(\left(n^{2}+n\right)-m\right)^{2} .
\end{aligned}
$$

Also ist diese Zahl für alle natürlichen Zahlen eine Quadratzahl.

L 23.3 Nach dem Satz des Pythagoras gelten folgende Gleichungen (Bezeichnungen siehe Skizze):

$$
\begin{aligned}
a^{2}+b^{2} & =210^{2} \mathrm{~m}^{2}=44100 \mathrm{~m}^{2} \\
b^{2}+c^{2} & =60^{2} \mathrm{~m}^{2}=3600 \mathrm{~m}^{2} \\
c^{2}+d^{2} & =180^{2} \mathrm{~m}^{2}=32400 \mathrm{~m}^{2} \\
d^{2}+a^{2} & =x^{2}
\end{aligned}
$$

Also gilt zum einen

$$
a^{2}+b^{2}+c^{2}+d^{2}=44100 \mathrm{~m}^{2}+32400 \mathrm{~m}^{2}
$$




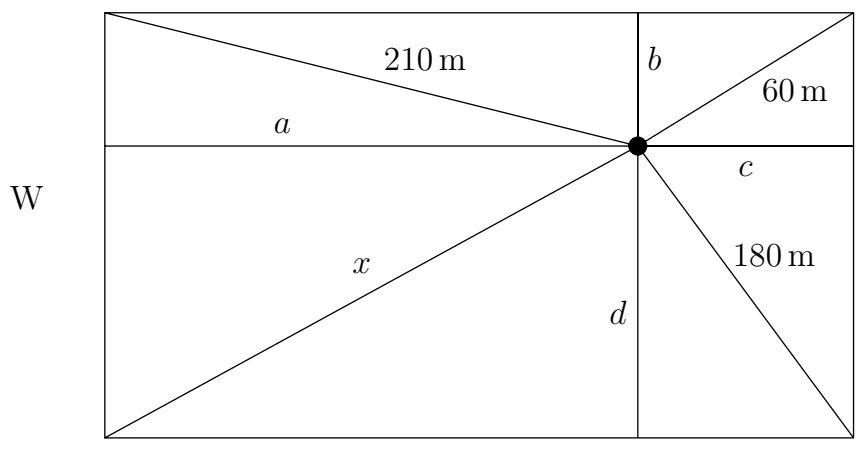

$\mathrm{O}$

S

Abbildung 23.1: Skizze des Anwesens des Freiherrn vom Kiesel

und zum anderen

$$
a^{2}+b^{2}+c^{2}+d^{2}=3600 \mathrm{~m}^{2}+x^{2}
$$

Damit: $x^{2}=44100 \mathrm{~m}^{2}+32400 \mathrm{~m}^{2}-3600 \mathrm{~m}^{2}=72900 \mathrm{~m}^{2}$.

Wegen $270^{2}=72900$ ist daher die gesuchte Länge: $x=270$ Meter.

L 23.4 Es sei aq $(n)$ die alternierende Quersumme der (natürlichen) Zahl $n$; sie wird gebildet, indem man von rechts beginnend die Ziffern der Zahl abwechselnd addiert und subtrahiert (anders gesagt: mit alternierendem Vorzeichen addiert). Beispiel: $\operatorname{aq}(44576)=6-7+5-4+4=0$.

Nun bedienen wir uns der Teilbarkeitsregel für 11 mit Hilfe der alternierenden Quersumme: Eine natürliche Zahl $n$ lässt beim Teilen durch 11 denselben Rest wie ihre alternierende Quersumme. Insbesondere ist eine Zahl genau dann durch 11 teilbar, wenn es ihre alternierende Quersumme ist. 
Die ersten gebildeten Zahlen müssen „von Hand“ getestet werden:

$$
\begin{aligned}
\operatorname{aq}(1) & =1, \\
\operatorname{aq}(12) & =1, \\
\mathrm{aq}(123) & =2, \\
\mathrm{aq}(1234) & =2, \\
\mathrm{aq}(12345) & =3, \\
\mathrm{aq}(123456) & =3, \\
\mathrm{aq}(1234567) & =4, \\
\mathrm{aq}(12345678) & =4, \\
\mathrm{aq}(123456789) & =5, \\
\mathrm{aq}(12345678910) & =4 .
\end{aligned}
$$

In den folgenden Schritten werden jeweils zwei Ziffern hinzugefügt. Das heißt, dass die vorher schon vorhandenen Ziffern denselben Anteil zur alternierenden Quersumme liefern wie beim Schritt vorher. Hinzugefügt wird der Anteil der neuen beiden Ziffern, und dies ist äquivalent dazu, den Rest beim Teilen durch 11 zu addieren. Es hat also

$$
\begin{aligned}
& 12 \ldots 1011 \text { denselben Rest wie } 4+0=4 \text {, } \\
& 12 \ldots 1112 \text { denselben Rest wie } 4+1=5 \text {, } \\
& 12 \ldots 1213 \text { denselben Rest wie } 5+2=7 \text {, } \\
& 12 \ldots 1314 \text { denselben Rest wie } 7+3=10 \text {, } \\
& 12 \ldots 1415 \text { denselben Rest wie } 10+4=14 \text {, dies entspricht 3, } \\
& 12 \ldots 1516 \text { denselben Rest wie } 3+5=8 \text {, } \\
& 12 \ldots 1617 \text { denselben Rest wie } 8+6=14 \text {, dies entspricht 3, } \\
& 12 \ldots 1718 \text { denselben Rest wie } 3+7=10 \text {, } \\
& 12 \ldots 1819 \text { denselben Rest wie } 10+8=18 \text {, dies entspricht } 7 \text {, } \\
& 12 \ldots 1920 \text { denselben Rest wie } 7+9=16 \text {, dies entspricht } 5 \text {, } \\
& 12 \ldots 2021 \text { denselben Rest wie } 5+10=15 \text {, dies entspricht } 4 \text {. }
\end{aligned}
$$

Da es jetzt wieder - wie elf Schritte zuvor - mit einer durch 11 teilbaren Zahl weitergeht und derselbe Rest vorliegt wie damals, wiederholt sich das Schema immer wieder, so dass unter den ersten 99 Zahlen keine durch 11 teilbare Zahl ist. 


\section{Lösungen zu Aufgabenblatt 24}

L 24.1 Zum Beweis geben wir für jedes $n \geq 6$ eine Zerlegung an.

Sei zunächst $n=2 m$ eine gerade Zahl mit $m \geq 3$. Dann ist folgende Zerlegung eines jeden Quadrates in $2 m$ kleinere Quadrate möglich:

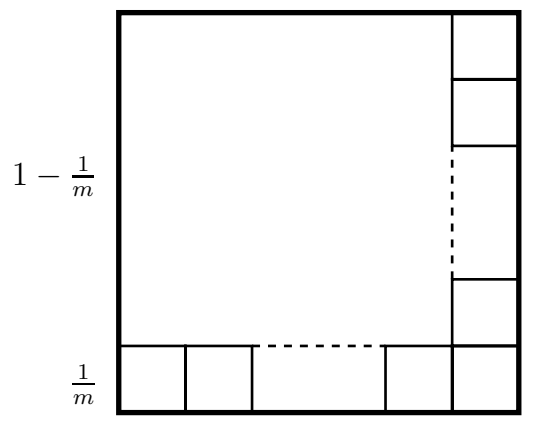

Dabei liegen unten bzw. rechts jeweils $m$ kleine Quadrate der Seitenlänge $\frac{1}{m}$ nebeneinander. Insgesamt haben wir das Quadrat somit in $2 m-1=n-1$ kleine Quadrate und ein größeres Quadrat (der Seitenlänge $1-\frac{1}{m}$ ) zerlegt.

Für ungerades $n=2 m+1$ mit $m \geq 3$ gibt es zum Beispiel folgende Zerlegung:

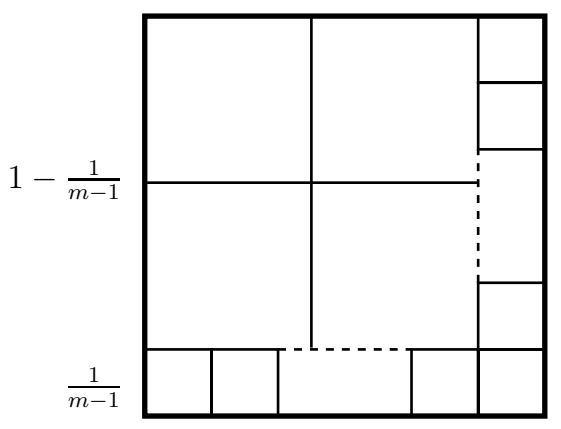

Diesmal haben die kleinen Quadrate die Seitenlänge $\frac{1}{m-1}$, so dass wir insgesamt $2(m-1)-1=n-4$ von ihnen erhalten. Das übrig bleibende große Quadrat wird in vier Quadrate unterteilt.

Damit ist alles gezeigt.

(Bemerkung: Es gibt natürlich noch viele weitere Möglichkeiten zum Zerlegen.) 
L 24.2 Entlang des Straßennetzes sind vertikale, horizontale und diagonale Bewegungen möglich. Hierbei können nur folgende Streckenlängen in Frage kommen, wobei als Einheit der Abstand zweier vertikal bzw. horizontal direkt benachbarter Häuser genommen wurde: $1, \sqrt{2}, 2,2 \sqrt{2}, 3,4,3 \sqrt{2}, 5,4 \sqrt{2}, 6,7,5 \sqrt{2}, 6 \sqrt{2}, 7 \sqrt{2}$. Wie man leicht nachrechnet, sind diese Längen schon nach wachsender Größe geordnet. Ein Weg maximaler Länge kann diese Längen also höchstens je einmal enthalten, ist demnach höchstens so lang wie die Summe dieser Strecken, d. h. $28 \cdot(1+\sqrt{2})$.

Nun wird gezeigt, dass bei einer erlaubten Route nicht alle der vier Streckenlängen $7,5 \sqrt{2}, 6 \sqrt{2}, 7 \sqrt{2}$ vorkommen können.

Kämen diese nämlich alle vor, so müssten sie in dieser Reihenfolge aufeinander folgend vorkommen. Der letzte Weg hätte daher die Länge $7 \sqrt{2}$; er verliefe somit von einer Ecke Schneebergs zur diagonal gegenüberliegenden Ecke. Der vorletzte und der drittletzte Weg könnten dann aber nur auf derselben Diagonalen verlaufen sein; der drittletzte Weg (der Länge $5 \sqrt{2}$ ) würde dann insbesondere in einem inneren Punkt (Nicht-Eckpunkt) der Diagonalen beginnen. Es gibt aber keinen Weg der Länge 7, der in einem solchen Punkt endet, denn die Länge 7 wird nur durch horizontale bzw. vertikale Wege von einem Rand des Ortes zum gegenüberliegenden Rand realisiert. Also können tatsächlich nicht alle vier der größtmöglichen Weglängen in einer Route vorkommen.

Eine erlaubte Route besteht daher höchstens aus 13 Wegen (und besucht 14 Häuser) und hat höchstens die Länge

$$
28 \cdot(1+\sqrt{2})-7=21+28 \sqrt{2}
$$

(da wir mindestens einen der Wege der Länge $\geq 7$ auslassen müssen, müssen wir von der Summe aller möglichen Teilstrecken mindestens die Länge 7 subtrahieren). Eine erlaubte Route mit diesen Werten wird im Folgenden angegeben.

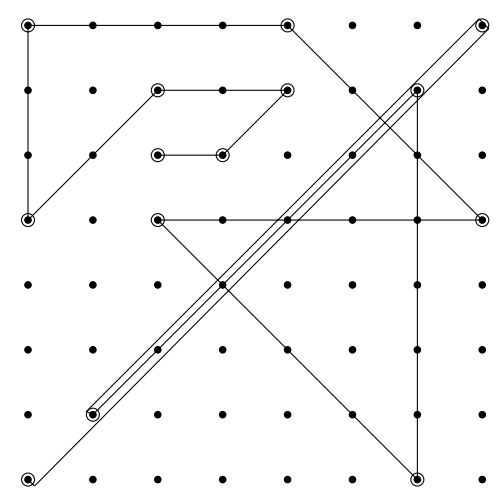

Abbildung 24.1: Eine Route der Länge $21+28 \sqrt{2}$, die 14 Häuser besucht 
Lösungen zu Aufgabenblatt 24

L 24.3 Zunächst einige Beispiele von magischen Quadraten:

\begin{tabular}{|c|c|c|}
\hline 729 & 2187 & 9 \\
\hline 3 & 243 & 19683 \\
\hline 6561 & 27 & 81 \\
\hline
\end{tabular}

\begin{tabular}{|c|c|c|}
\hline 5 & 1 & 25 \\
\hline 25 & 5 & 1 \\
\hline 1 & 25 & 5 \\
\hline
\end{tabular}

\begin{tabular}{|c|c|c|}
\hline 18 & 1 & 12 \\
\hline 4 & 6 & 9 \\
\hline 3 & 36 & 2 \\
\hline
\end{tabular}

Angenommen, wir haben ein multiplikativ-magisches Quadrat mit den ganzzahligen Einträgen $a$ bis $j$ wie in folgendem Bild und mit magischer Konstante $N>0$ :

\begin{tabular}{|l|l|l|}
\hline$a$ & $b$ & $c$ \\
\hline$d$ & $e$ & $f$ \\
\hline$g$ & $h$ & $j$ \\
\hline
\end{tabular}

Dann gelten insbesondere die folgenden Gleichungen:

$$
\begin{aligned}
a b c & =N \\
g h j & =N \\
\text { aej } & =N \\
\text { beh } & =N \\
c e g & =N .
\end{aligned}
$$

Multipliziert man die letzten drei Gleichungen, so erhält man:

$$
(a b c)(g h j) e^{3}=N^{3}
$$

Zusammen mit den ersten beiden Gleichungen folgt daraus direkt $e^{3}=N$. Also ist $N$ eine Kubikzahl.

(Bemerkung: Übrigens kann man auf ganz analogem Wege zeigen, dass die magische Konstante eines additiv-magischen Quadrates durch 3 teilbar ist.) 
L 24.4 Zunächst kann man ein solches Zerlege-Rechteck in $(n+1) n$ Rechtecke mit den Seitenlängen $\frac{a}{n+1}$ und $\frac{b}{n}$ unterteilen, indem man die Seiten $a$ und $b$ in $n+1$ bzw. $n$ gleiche Teile teilt. Auf dem dadurch entstehenden Gitter zerschneide man das Rechteck in Treppenform, wie es in folgendem Bild für den Fall $n=3$ angedeutet ist:

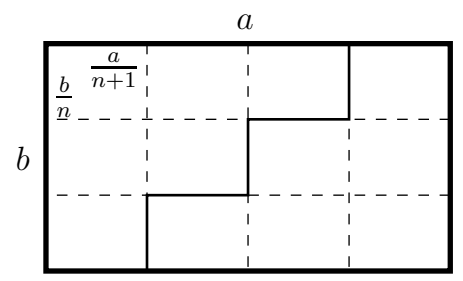

Verschiebt man nun das rechte Teil um eine "Treppenstufe“ nach links unten, so erhält man offenbar ein neues Rechteck:

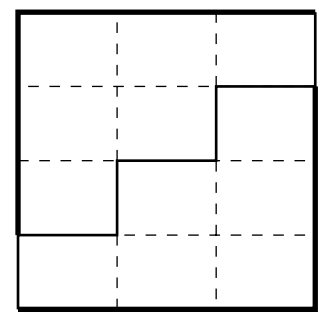

Im Fall eines allgemeinen $n$ hat dieses dann die Seitenlängen $n \cdot \frac{a}{n+1}$ und $(n+1) \cdot \frac{b}{n}$. Wegen $\frac{a}{b}=\frac{(n+1)^{2}}{n^{2}}$ gilt $n \cdot \frac{a}{n+1}=(n+1) \cdot \frac{b}{n}$. Daher ist das aus den beiden Teilen neu zusammengesetzte Rechteck ein Quadrat. 


\section{Lösungen zu Aufgabenblatt 25}

L 25.1 Seien $B, R$ und $S$ die Preise von Bleistift, Radiergummi bzw. Spitzer in Cent. Dann erhält man aus dem Text folgende Gleichung und Ungleichungen:

$$
\begin{aligned}
B+R+S & =100 \\
S & >2 \cdot B \\
3 \cdot B & >4 \cdot R \\
3 \cdot R & >S .
\end{aligned}
$$

Aus den Ungleichungen (25.2) und (25.3) erhält man noch

$$
S>\frac{8}{3} \cdot R
$$

und aus (25.2) und (25.4) erhält man

$$
B<\frac{3}{2} \cdot R
$$

Damit gilt:

$$
\begin{aligned}
100=B+R+S & >\frac{4}{3} \cdot R+R+\frac{8}{3} \cdot R=5 \cdot R \\
& \Longrightarrow \quad 20>R
\end{aligned}
$$

und

$$
\begin{aligned}
100=B+R+S & <\frac{3}{2} \cdot R+R+3 \cdot R=\frac{11}{2} \cdot R \\
& \Longrightarrow \quad R \quad>\frac{200}{11}=18+\frac{2}{11} .
\end{aligned}
$$

Da $R$ ganzzahlig ist, ist somit $R=19$.

Nun berechnen wir $S$ durch Abschätzen von oben und von unten:

$$
\begin{aligned}
100=B+R+S & >\frac{4}{3} \cdot 19+19+S \\
& \Longrightarrow \quad S \quad<100-\frac{4}{3} \cdot 19-19=55+\frac{2}{3}
\end{aligned}
$$

und

$$
\begin{aligned}
100=B+R+S & <\frac{1}{2} \cdot S+19+S \\
\Longrightarrow \quad S & >\frac{2}{3} \cdot(100-19)=54 .
\end{aligned}
$$


Somit gilt $S=55$. Aus der Gleichung (25.1) folgt nun: $B=100-19-55=26$.

Eine Probe zeigt, dass $B=26, R=19$ und $S=55$ alle drei Ungleichungen erfüllen.

L 25.2 Um die Plings besser zählen zu können, stellen wir uns die Situation ein wenig anders vor: Wenn zwei Kugeln zusammenstoßen, erzeugen sie nicht nur ein Pling, sondern sie tauschen (auf welche Weise auch immer) blitzschnell die Plätze und rollen dann einfach in ihrer vorherigen Richtung weiter.

Die Konfiguration der Kugeln mit dieser neuen Eigenschaft im Rohr ist nun zu jedem Zeitpunkt dieselbe, wie die Konfiguration der Kugeln wäre, wenn sie sich ganz normal abstoßen würden.

Dies wird sofort klar, wenn man sich überlegt, dass in beiden Fällen vor einem Pling zwei Kugeln mit einer gewissen (gleichen, aber entgegengesetzten) Geschwindigkeit aufeinander zu rollen und nach dem Pling (das in beiden Fällen zum gleichen Zeitpunkt ertönt) zwei Kugeln mit derselben Geschwindigkeit vom Ort der Erzeugung des Plings in entgegengesetzter Richtung wegrollen.

Also ist die zu bestimmende Anzahl an Plings in beiden Szenarien dieselbe. In dem Fall, dass die Kugeln einfach ihre Positionen tauschen, ist aber klar, dass jede der 29 von links kommenden Kugeln genau auf 29 von rechts kommende Kugeln trifft. Es gibt also genau $29 \cdot 29=841$ Plings.

L 25.3 Bezeichnen wir die Ecken des Dreiecks mit $A, B$ und $C$ wie in der Skizze.

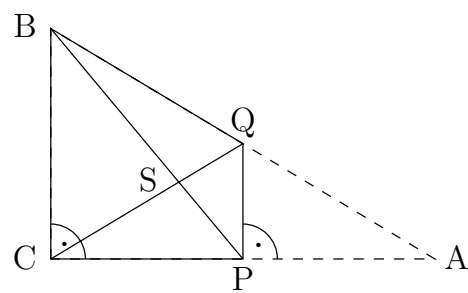

Die Ecke $A$ werde nun auf die Ecke $C$, bei der der rechte Winkel liegt, gefaltet. Die Punkte $P$ und $Q$ auf den Seiten $A C$ bzw. $A B$ sollen auf der Faltkante liegen. Der Punkt $S$ sei der Schnittpunkt der Geraden $(B Q)$ und $(C Q)$. Da beim Falten $A$ auf $C$ zu liegen kommt, ist $P$ der Mittelpunkt der Strecke $A C$ und die Gerade $(P Q)$ ist senkrecht zu $A C$. Somit ist $(P Q)$ parallel zur Strecke $B C$.

Nach dem zweiten Strahlensatz ist daher $\overline{P Q}: \overline{A P}=\overline{C B}: \overline{A C}$, d. h. $\overline{P Q}=\frac{1}{2} \cdot \overline{C B}$.

Nach dem zweiten Strahlensatz (jetzt mit Zentrum $S$ ) gilt weiter: $\overline{Q P}: \overline{S Q}=\overline{B C}: \overline{S C}$, d. h. $\overline{S Q}=\frac{1}{2} \cdot \overline{S C}$.

Genauso (oder nun nach dem ersten Strahlensatz) gilt: $\overline{S P}=\frac{1}{2} \cdot \overline{S B}$.

Die Diagonalen schneiden sich also jeweils im Verhältnis $2: 1$. 
Lösungen zu Aufgabenblatt 25

L 25.4 Sei $n$ eine der gesuchten Zahlen und seien ihre Ziffern $a, b, c, d$ und $e$. Es ist also $n=10000 a+1000 b+100 c+10 d+e$. Die Zahl, die man durch Streichen der mittleren Ziffer $(c)$ erhält, ist somit: $m:=1000 a+100 b+10 d+e$.

Diese ist jetzt ein Teiler von $n$ oder gleichbedeutend dazu ist $n$ ein Vielfaches von $m$.

Nun gilt aber:

$$
\begin{aligned}
11 \cdot m & =11000 a+1100 b+110 d+11 e \\
& =n+1000 a+100 b+100 d+10 e-100 c>n,
\end{aligned}
$$

denn $1000 a+100 b+100 d+10 e \geq 1000>100 c$.

Außerdem:

$$
9 \cdot m=9000 a+900 b+90 d+9 e=n-1000 a-100 b-100 c+80 d+8 e<n,
$$

denn $1000 a+100 b+100 c \geq 1000>800+80>80 d+8 e$.

Damit gilt: $n=10 \cdot m=10000 a+1000 b+100 d+10 e$ und daher erhalten wir durch Vergleichen der Ziffern: $e=0, d=e$ und $c=d$.

Die einzigen Zahlen $n$, die die Bedingung erfüllen können, enden also auf drei Nullen. Wie man sofort sieht, ist für diese Zahlen die Bedingung aber auch erfüllt.

Also sind dies alle fünfstelligen Zahlen, die die Bedingung erfüllen. 


\section{Index}

Abenteurer, A16.3

Abstand

der Kneipen, A14.2

Abstandssumme minimale, A12.2

Abstellgleis, A20.1

Altstadtfest, A6.2

Appolonius Kreis des, A18.3

Armbänder, A12.3

Asterix, A18.4, A19.4

Aufteilen

Schatz, A2.1

Schokolade, A1.2

Aussagenlogik, A15.3, A19.1

Balkenwaage, A13.4

Basketball, A15.3

Bauarbeiter, A7.2

Bauer Hauser, A22.3

Benfords Gesetz, A6.4

Bettdecke, A1.4

Binomialkoeffizient, A9.3

Bleistift, A25.1

Bluthochdruck, A3.1

Bowlingkugeln, A25.2

Brüche, A5.1

Buch der Wahrheit, A19.1

Buffons Nadelproblem, A2.4

Chauffeur, A1.1

Code, A6.3

Deutschland Mittelpunkt, A12.4

Diagonalen, A25.3

Differenzen, A22.4

DIN-Format, A7.1

Dominosteine, A17.2

Dreieck gleichschenkliges, A21.2 gleichsseitiges, A12.2 rechtwinkliges, A13.3, A25.3

Dreiecksungleichung, A14.1

Drillinge, A13.1

E-Mail, A5.3

Eiche, A23.3

Eis laufen, A16.1

Eisenbahnnetze, A21.3

Euklidischer Algorithmus, A7.3

Euler, A7.4

Extremalprinzip, A5.3, A16.4

Fähnlein Fieselschweif, A4.2

Färbungsprinzip, A8.4

Fakultät, A17.4, A21.1

Falten, A25.3

Familie Wurm, A1.4

Farbe, A8.3

First-Digit-Gesetz, A6.4

Fläche, A21.2

Folge

arithmetische, A21.1

Frauenstammtisch, A5.3

Freiherr vom Kiesel, A23.3

Frosch, A7.3

Futter, A16.4

Geburtstagsparty, A23.1

Geometrie, A6.2, A8.2, A9.4, A10.3, A17.1, $\mathrm{A} 18.3, \mathrm{~A} 19.3, \mathrm{~A} 21.2, \mathrm{~A} 22.3, \mathrm{~A} 24.2$ am Dreieck, A12.2, A13.3, A25.3

am Rechteck, A23.3, A24.4

am Zylinder, A2.2

auf der Kugel, A16.3

experimentelle, A1.4, A4.4, A10.4

kombinatorische, A5.4, A11.2, A11.4,

A12.4, A14.1, A15.4, A17.3, A24.1

mit Dreieck und Kreisen, A4.3

mit Kreis und Dreiecken, A3.3

topologische, A16.1

Geometrischer Ort, A3.3

Gewinnstellung, A11.3 
$\operatorname{ggT}, \mathrm{A} 7.3$

Gitterwege, A14.4

Gleichung, A1.1, A4.2, A7.1, A7.2, A9.1, A13.2, A13.4, A14.2, A24.3, A25.1 mit Betrag, A10.3

quadratische, A8.2, A10.4

Glückszahl, A22.2

Göttinger Fernsehturm, A2.2

Grassoden, A8.4

Grillen, A21.4

Haufen, A11.3

Herr Schluckspecht, A10.1, A14.2

Herr Schulze, A1.1

Höhenschnittpunkt, A3.3

Hölzchenspiel, A11.3

Holzstäbchen, A14.1

Holzwürfel, A18.2

Inkreisradius, A13.3

Invariantenprinzip, A8.3

Kaffee, A13.2

Kassette, A10.4

Kerzen, A13.4

Kohlenkeller, A4.4

Kombinatorik, A5.3, A6.3, A8.3, A8.4, A11.3, A13.2, A13.4, A14.4, A16.4, A17.2, A18.4, A19.4, A20.1, A21.4, A23.1, A25.2

abzählende, A9.3, A12.3, A21.3, A22.1

geometrische, A18.2

mit Schach, A3.4, A19.2

mit Spielkarten, A7.4

Kryptogramm, A11.1

Kubikzahl, A24.3

Kurven, A11.2

Leiter, A8.2

Liegestuhl, A12.2

Linien, A22.1

Logik, A4.1, A10.1, A15.3, A19.1

Lok, A20.1

Mädchenanteil, A15.1

Meridian, A16.3
Mikadospiel, A14.1

Milch, A13.2

Mittelpunkt Deutschlands, A12.4

Möwenland, A21.3

Münzwerfen, A18.4, A19.4

n-Frosch, A7.3

Nachtwächter, A11.4

Nadel, A2.4

NENA, A11.1

Neu Weende, A11.2

Obelix, A18.4, A19.4

Oma Piepenbrink, A8.1

Optimierung, A21.4

Palindrome, A1.3

Parkettierung, A9.4

Paul Sorglos, A11.4

Perlen, A12.3

$\pi, \mathrm{A} 2.4$

Pling, A25.2

Polygon, A11.4, A19.3

Potenzsummen, A2.3

Primzahl, A9.2, A16.2, A20.4

Primzahlzwilling, A16.2

Pythagoras

Satz des, A2.2, A8.2, A17.1, A23.3

Quadrat, A5.4, A21.2, A24.1, A24.4

Eulersches, A7.4

lateinisches, A7.4

magisches, A24.3

Rasenfläche, A8.4

Quadratzahl, A6.1, A10.2, A14.3, A18.1, A23.2

Quersumme, A6.1

alternierende, A23.4

Radiergummi, A25.1

Rätsel, A20.4

Rahmen, A19.3

Rentier Rudolf, A24.2

Rentieriade, A16.4

Reporter, A16.3

Rückwärtsrechnen, A2.1, A12.1 
Sand schaufeln, A7.2

Sandburg, A18.3

Satz von Pick, A14.4

Schachbrett mit Figuren, A3.4

Schmuckbänder, A6.2

Schneewittchen, A9.3

Schokoladentafel, A1.2

Schubfachprinzip, A21.3, A23.1

Seeräuber, A2.1

Sehnenviereck, A3.3, A4.3

Spiel

mit Rückwärtsrechnen, A12.1

Spielkarten, A7.4

Spielsteine, A8.3

Spitzer, A25.1

Stammbruch, A21.1

Steaks, A21.4

Stochastik, A2.4

Strahlensatz, A6.2, A22.3

Straßennetz, A24.2

Supermarktpreise, A6.4

Taschengeld, A8.1

Teilbarkeit, A13.1, A17.4, A23.4

Teile

kongruente, A17.3

zählen, A1.2

Teilen mit Rest, A3.2

Teiler, A25.4

Thales

Satz des, A13.3

Tick, Trick und Track, A4.2

Türme, A19.2

Tunnel, A20.1

Uhrzeiger, A9.1

Umkreisradius, A13.3

Ungleichung, A5.1, A15.1, A25.1

Verbrecher, A4.1

Verhältnis, A25.3

Verluststellung, A11.3

Wagen, A20.1

Wahrscheinlichkeiten abhängige, A3.1

Wahrscheinlichkeitsrechnung, A10.1, A18.4, A19.4

Wecker, A13.4

Weg

$$
\text { kürzester, A11.2 }
$$

Weglänge, A10.3

Weiberheld, A10.3

Weihnachtsmann, A24.2

Weltliniendiagramm, A1.1, A4.2

Wendeltreppe, A2.2

Wurm, A1.4

Wurzelterm, A22.2

Zahl

fünfstellige, A25.4

sechsstellige, A15.2, A18.1

Zahlenrätsel, A20.2

Zahlenschloss, A6.3

Zahlentheorie, A2.1, A3.2, A5.2, A6.1, A7.3, $\mathrm{A} 9.2, \mathrm{~A} 10.2, \mathrm{~A} 13.1, \mathrm{~A} 14.3, \mathrm{~A} 15.2$, A16.2, A17.4, A18.1, A20.4, A21.1, $\mathrm{A} 22.2, \mathrm{~A} 23.2, \mathrm{~A} 23.4, \mathrm{~A} 24.3, \mathrm{~A} 25.4$

experimentelle, A2.3, A6.4

kombinatorische, A1.3, A8.1, A20.3, A22.4

Zerlege-Rechteck, A24.4

Zerlegung, A15.4, A17.3, A18.2, A24.1

Zerschneiden, A5.4

Ziffer

fehlende, A15.2, A17.4

gleiche, A10.2

Häufigkeit, A6.4

ordnen, A20.3

Ziffernverstellung, A5.2

Zwerge

sieben, A9.3

21, A20.2 



\section{Inhaltsverzeichnis}

Vorwort 1

Über den Korrespondenzzirkel 3

Anmeldecoupon $\quad 4$

I Aufgaben 5

$\begin{array}{ll}\text { Aufgabenblatt } 1 & 7\end{array}$

$\begin{array}{lr}\text { Aufgabenblatt } 2 & 9\end{array}$

Aufgabenblatt $3 \quad 11$

Aufgabenblatt $4 \quad 13$

$\begin{array}{ll}\text { Aufgabenblatt } 5 & 15\end{array}$

$\begin{array}{ll}\text { Aufgabenblatt } 6 & 17\end{array}$

$\begin{array}{lr}\text { Aufgabenblatt } 7 & 19\end{array}$

Aufgabenblatt $8 \quad 21$

$\begin{array}{ll}\text { Aufgabenblatt } 9 & 23\end{array}$

Aufgabenblatt $10 \quad 25$

Aufgabenblatt $11 \quad 27$

Aufgabenblatt $12 \quad 29$

Aufgabenblatt $13 \quad 31$

Aufgabenblatt $14 \quad 33$

Aufgabenblatt $15 \quad 35$

$\begin{array}{ll}\text { Aufgabenblatt } 16 & 37\end{array}$

$\begin{array}{ll}\text { Aufgabenblatt } 17 & 39\end{array}$

Aufgabenblatt $18 \quad 41$

Aufgabenblatt $19 \quad 43$

Aufgabenblatt $20 \quad 45$

$\begin{array}{ll}\text { Aufgabenblatt } 21 & 47\end{array}$

$\begin{array}{lr}\text { Aufgabenblatt } 22 & 49\end{array}$

Aufgabenblatt 23

Aufgabenblatt 24

Aufgabenblatt $25 \quad 55$ 


\section{Lösungen}

Lösungen zu Aufgabenblatt $1 \quad 59$

$\begin{array}{ll}\text { Lösungen zu Aufgabenblatt } 2 & 67\end{array}$

Lösungen zu Aufgabenblatt $3 \quad 74$

Lösungen zu Aufgabenblatt $4 \quad 78$

Lösungen zu Aufgabenblatt 5

Lösungen zu Aufgabenblatt $6 \quad 88$

Lösungen zu Aufgabenblatt $7 \quad 94$

Lösungen zu Aufgabenblatt $8 \quad 99$

Lösungen zu Aufgabenblatt $9 \quad 104$

Lösungen zu Aufgabenblatt $10 \quad 109$

Lösungen zu Aufgabenblatt $11 \quad 112$

Lösungen zu Aufgabenblatt $12 \quad 120$

Lösungen zu Aufgabenblatt $13 \quad 127$

Lösungen zu Aufgabenblatt $14 \quad 132$

Lösungen zu Aufgabenblatt $15 \quad 138$

Lösungen zu Aufgabenblatt $16 \quad 141$

$\begin{array}{lr}\text { Lösungen zu Aufgabenblatt } 17 & 144\end{array}$

Lösungen zu Aufgabenblatt $18 \quad 148$

Lösungen zu Aufgabenblatt $19 \quad 151$

Lösungen zu Aufgabenblatt $20 \quad 155$

$\begin{array}{ll}\text { Lösungen zu Aufgabenblatt } 21 & 157\end{array}$

Lösungen zu Aufgabenblatt $22 \quad 162$

Lösungen zu Aufgabenblatt $23 \quad 165$

Lösungen zu Aufgabenblatt $24 \quad 168$

Lösungen zu Aufgabenblatt 25 172

$\begin{array}{ll}\text { Index } & 175\end{array}$ 